University of Louisville

ThinkIR: The University of Louisville's Institutional Repository

Electronic Theses and Dissertations

$12-2020$

\title{
Development of a Pediatric cardiac assist Maglev pump for use with a universal driver system.
}

Landon Heath Tompkins

University of Louisville

Follow this and additional works at: https://ir.library.louisville.edu/etd

Part of the Biomedical Devices and Instrumentation Commons, Computer-Aided Engineering and Design Commons, and the Translational Medical Research Commons

\section{Recommended Citation}

Tompkins, Landon Heath, "Development of a Pediatric cardiac assist Maglev pump for use with a universal driver system." (2020). Electronic Theses and Dissertations. Paper 3542.

https://doi.org/10.18297/etd/3542

This Doctoral Dissertation is brought to you for free and open access by ThinkIR: The University of Louisville's Institutional Repository. It has been accepted for inclusion in Electronic Theses and Dissertations by an authorized administrator of ThinkIR: The University of Louisville's Institutional Repository. This title appears here courtesy of the author, who has retained all other copyrights. For more information, please contact thinkir@louisville.edu. 


\title{
DEVELOPMENT OF A PEDIATRIC CARDIAC ASSIST MAGLEV PUMP FOR USE WITH A UNIVERSAL DRIVER SYSTEM
}

\author{
By \\ Landon Heath Tompkins \\ B.S., University of Louisville, 2010 \\ MEng., University of Louisville, 2011 \\ A Dissertation Submitted to the \\ Graduate School, University of Louisville, \\ in Partial Fulfillment of the Requirements \\ for the Degree of
}

Doctor of Philosophy in Interdisciplinary Studies with Specialization in Translational Bioengineering

Department of Bioengineering

J.B. Speed School of Engineering

University of Louisville

Louisville, Kentucky

December 2020 

DEVELOPMENT OF A PEDIATRIC CARDIAC ASSIST MAGLEV PUMP FOR USE WITH A UNIVERSAL DRIVER SYSTEM

\author{
By \\ Landon Heath Tompkins \\ B.S., University of Louisville, 2010 \\ MEng., University of Louisville, 2011 \\ A Dissertation Approved on
}

November 24, 2020

by the following Dissertation Committee:

Steven C. Koenig, Ph.D.

Kurt A. Dasse, Ph.D.

Mark S. Slaughter, M.D.

Thomas Roussel, Ph.D.

Jonathan A. Kopechek, Ph.D.

Stuart J. Williams, Ph.D. 


\section{ACKNOWLEDGEMENTS}

There are so many friends, teachers, mentors, and colleagues I've had in my life who have made the completion of this dissertation possible, and I wish to thank all of them from the bottom of my heart. But above all, l'd like to express my deepest gratitude and thanks to the following individuals whose contributions to my success have been immeasurable.

To Daiga Koenig, for her endless encouragement, support, and friendship. If not for her persistent belief, I never would have shifted my career to continue my education, but I'm sure glad it worked out this way. It has been the most rewarding experience of my life. Thanks Dog.

To Dr. Steven Koenig, my chair, mentor, and friend. You have pushed me to accomplish things I never would have considered or imagined, and it is because of you that I've become the engineer and researcher I am today. I don't think I can adequately express with words the impact you've had on my life, but l'll just start by saying thank you.

To Dr. Kurt Dasse for his support, mentorship, and wisdom. I am incredibly fortunate to have you in my corner and I can't wait to see what the next chapter brings. To Dr. Mark Slaughter for his support over the past many years and for always asking the tough questions (which always push me to do better). To Dr. Thomas Roussel, Dr. Jonathan Kopechek, and Dr. Stuart Williams for 
their support, guidance, and contributions to this research and my graduate career. I have so enjoyed getting to work with all of you and am extremely grateful you were part of my committee.

To Dr. Paul Spence for providing me an unparalleled education and for helping me find focus in my life. To all the amazing individuals I've had the privilege of working with throughout the years in the Advanced Heart Failure Research group: Todd Adams (my bro), Mike Sobieski, Dr. Gretel Monreal, Abhi, Conner, and Natalie. To the amazing development team who have helped make the Inspired Pediatric VAD a reality, Gino Morello, Dr. Steven Prina, and Barry Gellman. You guys are awesome.

Above all l'd like to thank my parents for providing me every opportunity in my life. Without them none of this would be possible. To my family, Meemaw, Seth, Nicole, and baby Idris, I love you all. To Pappy for being so, so proud.

To my best friend. I'm so glad that, even after all of this is over, I still get to spend the rest of my life with you. And, to Linus and Truman, for their unwavering and unparalleled moral support. 


\section{ABSTRACT \\ DEVELOPMENT OF A PEDIATRIC CARDIAC ASSIST MAGLEV PUMP FOR USE WITH A UNIVERSAL DRIVER SYSTEM \\ Landon H. Tompkins}

November 24, 2020

Heart failure (HF) remains the leading cause of death, affecting 26 million adults worldwide and 6.5 million adults in the United States. Pediatric HF patients have been a historically underserved population with few options for mechanical circulatory support (MCS) therapy, a leading treatment as an alternative to heart transplantation. To address this clinical need, the Inspired Universal MagLev System is being developed; a low cost, universal magnetically levitated extracorporeal MCS system with interchangeable single-use pumps that will ultimately provide adult and pediatric patients ventricular and respiratory assist therapies. The Inspired Pediatric VAD is the first single-use pump application for this MCS system and is specifically designed for pediatric circulatory support. This dissertation describes the development efforts to design and evaluate iterative impeller and pump housings for the Inspired Pediatric VAD.

Requirements for the Inspired Pediatric VAD design include the need to generate the appropriate hemodynamic parameters (pressures and flows) for pediatric patients, and miniaturization of the pump and impeller to accommodate the pediatric population. Traditional pump theory and design methods were 
applied to aid in the unique design of the VAD impeller and pump housing, resulting in multiple design iterations. Two impeller and pump designs (V1, V2) were virtually constructed using computer-aided design (CAD) software. Threedimensional flow and pressure features were analyzed using computational fluid dynamics (CFD) analysis. Simulated pump designs (V1, V2) were operated at $15 \%$ higher rotational speeds ( $5000 \mathrm{rpm})$ than initially estimated $(4255 \mathrm{rpm})$ to achieve the desired operational point $(3.5 \mathrm{~L} / \mathrm{min}$ flow at $150 \mathrm{mmHg}) . V 2$ design outperformed $V 1$ by generating up to $30 \%$ higher pressures at all simulated rotational speeds and with $5 \%$ lower priming volume. Simulated hydrodynamic performance (flow, pressure and hydraulic efficiency) of VAD V2 compared favorably to current commercially available MCS devices.

A prototype of the Inspired Pediatric VAD V2 was fabricated, the magnitude and range of hydraulic torque and forces of the impeller were quantified, and the hydrodynamic performance benchmarked. A static mock flow loop model containing a heated blood analogue solution was created to test the pump over a range of rotational speeds (500 - 6000 RPM), flow rates (0 - 3.5 $\mathrm{L} / \mathrm{min}$ ), and pressures ( 0 to $\sim 420 \mathrm{mmHg}$ ). The device was initially powered by a shaft driven DC motor, which was used to calculate the fluid torque acting on the impeller. Additional CFD simulations of VAD V2 were compared against the empirical bench-top data at select rotational speed and flow rate conditions. Empirically, the pediatric $V A D$ produced flows as high as $4.3 \mathrm{~L} / \mathrm{min}$ against a pressure of $127 \mathrm{mmHg}$ at $6000 \mathrm{RPM}$. 
Based on the performance of the first two VAD design iterations, a final design iteration, $V A D V 3$, was achieved. Hydrodynamic performance of $V A D V 3$ was numerically assessed using CFD simulations. The results indicated no change in flow and pressure head performance compared to the previous device design (V2). Shear stress and flow residence time volumetric distributions were generated over a range of pump rotational speeds and flow rates. At the lowest pump operating point (3000 RPM, $0.50 \mathrm{~L} / \mathrm{min}, 75 \mathrm{mmHg}$ ), $79 \%$ of the pump volume was in the shear stress range of $0-10 \mathrm{~Pa}$ with $<1 \%$ of the volume in the critical range of $150-1000 \mathrm{~Pa}$ associated with potential for increased risk of clinically-significant blood damage. At higher speed and flow (5000 RPM, 3.50 $\mathrm{L} / \mathrm{min}, 176 \mathrm{mmHg}$ ), $65 \%$ of the volume resided in the $0-10 \mathrm{~Pa}$ range compared to $2.3 \%$ at $150-1000 \mathrm{~Pa}$. The initial results from the computational characterization of the Inspired Pediatric VAD V3 were encouraging, and based on the overall research performed to date, future work will include pre-clinical testing of VAD V3 in static and dynamic mock flow loop and acute large animal model studies to further assess device function, hydrodynamic performance, hemodynamic response, and hemocompatibility. 


\section{TABLE OF CONTENTS}

$\underline{\text { Page }}$

ACKNOWLEDGEMENTS ..............................................................................

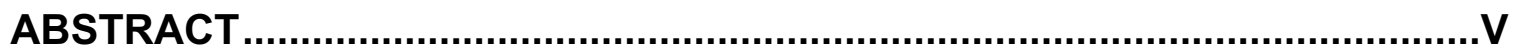

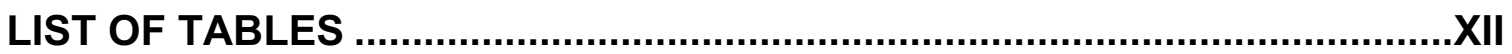

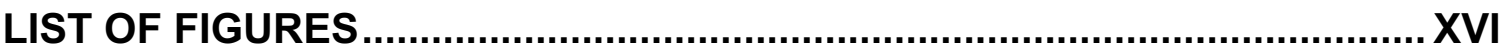

CHAPTER $1:$ INTRODUCTION...................................................................... 1

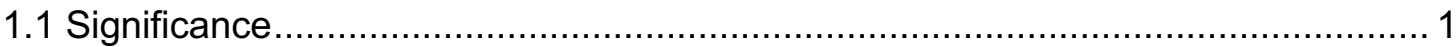

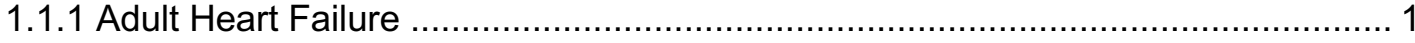

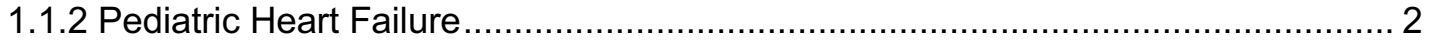

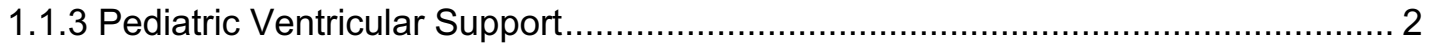

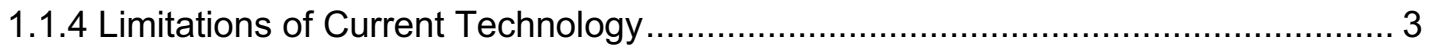

1.1.5 Inspired Pediatric VAD and Universal MagLev System .................................... 4

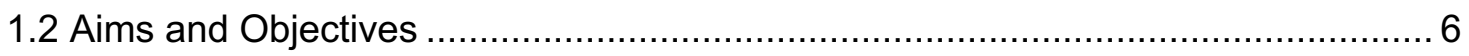

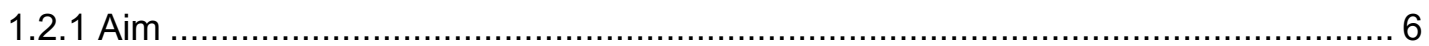

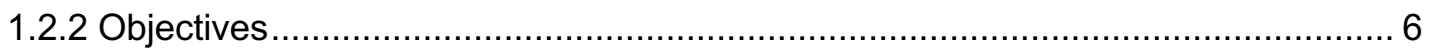

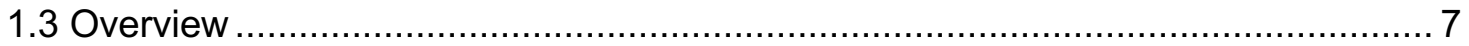

CHAPTER 2 : IMPELLER DESIGN OF AN EXTRACORPOREAL PEDIATRIC LVAD FOR USE WITH INSPIRED UNIVERSAL MAGLEV SYSTEM ........................................ 10

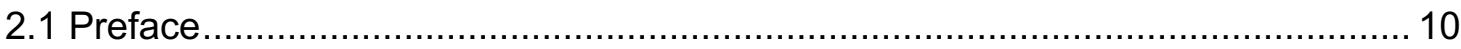

2.2 Impeller Rotational Speed Estimation .................................................... 12

2.3 Determination of Pump Specific Speed ................................................. 14

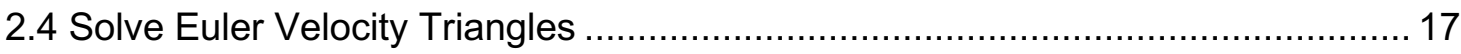

2.5 Account for Flow Slip, Recirculation, and Pre-Rotation ................................. 22

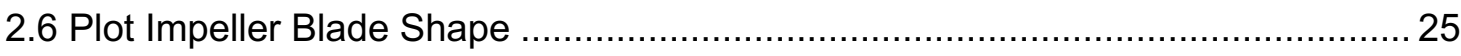

2.7 Impeller Blade Thickness and Profiling..................................................... 28

2.8 Additional Impeller Features .................................................................... 31 


\section{CHAPTER 3 : DESIGN AND COMPUTATIONAL EVALUATION OF A PEDIATRIC MAGLEV ROTARY BLOOD PUMP ...................... 35}

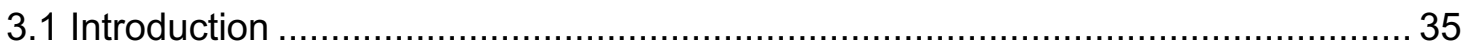

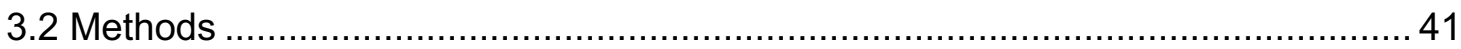

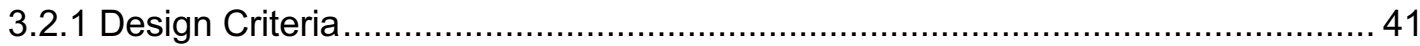

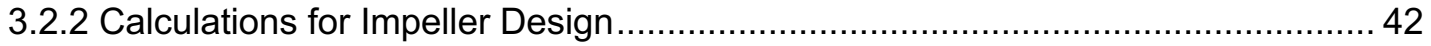

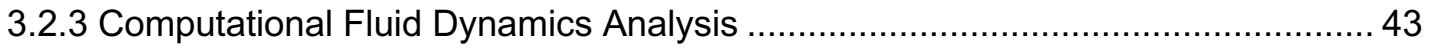

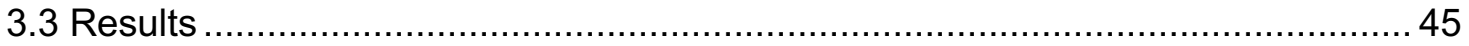

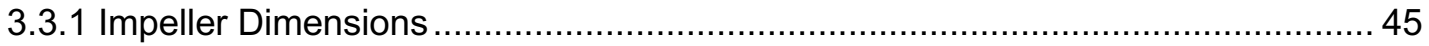

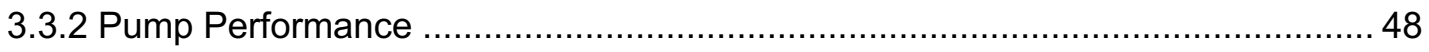

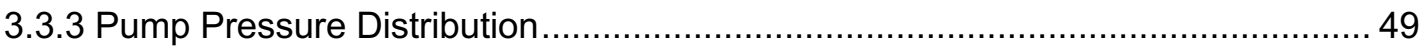

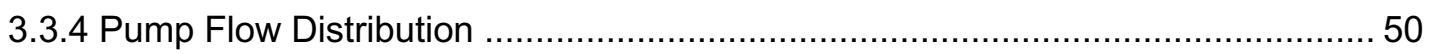

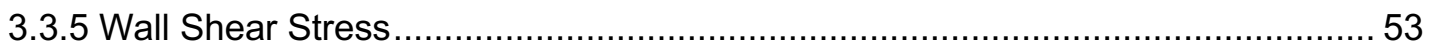

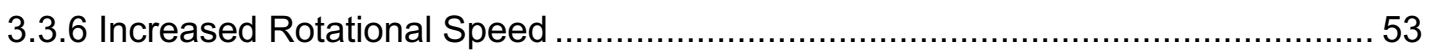

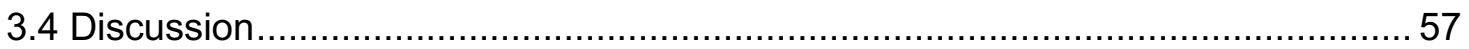

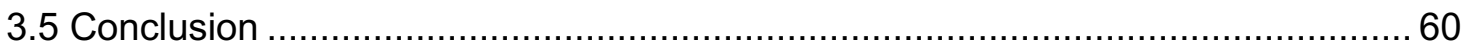

\section{CHAPTER 4 : DEVELOPMENT OF INSPIRED THERAPEUTICS} PEDIATRIC VAD: QUANTIFYING IMPELLER TORQUES AND FORCES FOR MAGLEV MOTOR DESIGN .....................62

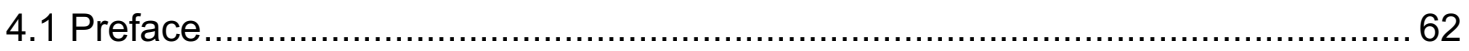

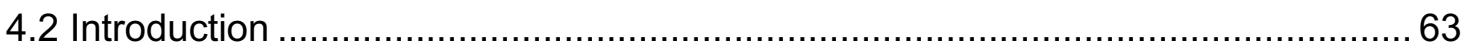

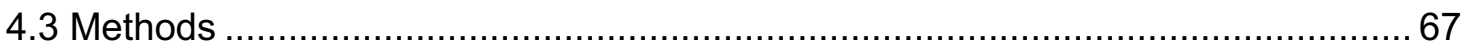

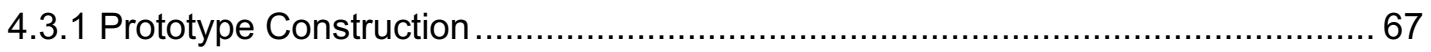

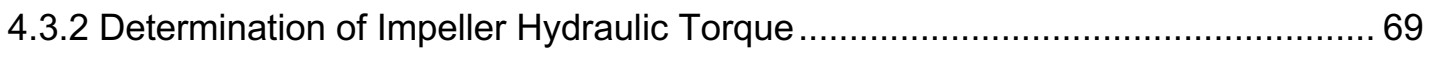

4.3.3 Static Flow Loop (SFL) Model Testing …................................................... 72

4.3.4 Computational Fluid Dynamics (CFD) Model Simulations ................................. 74

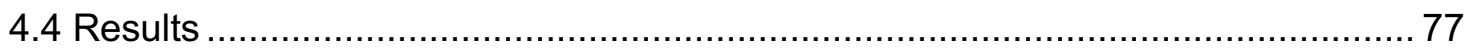

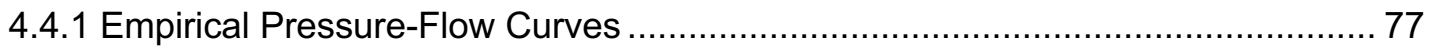

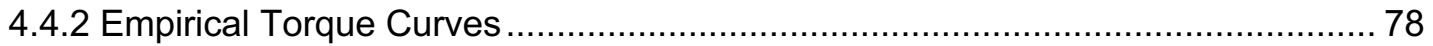

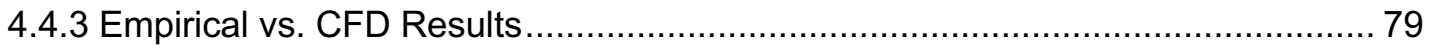

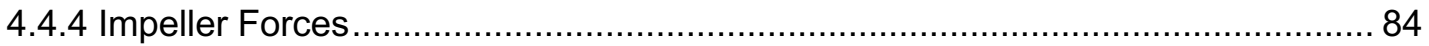

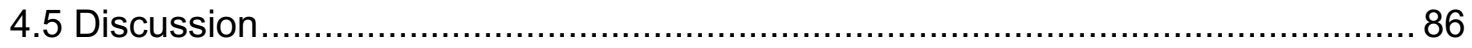

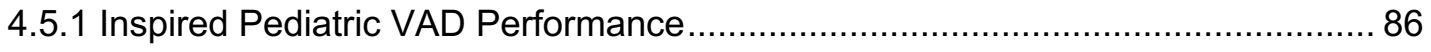




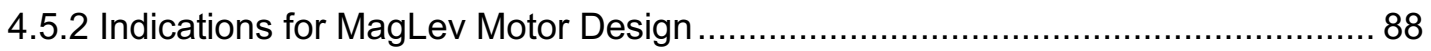

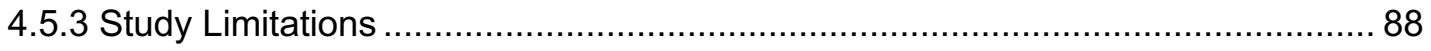

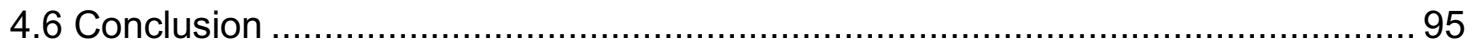

CHAPTER 5 : DEVELOPMENT OF INSPIRED THERAPEUTICS PEDIATRIC VAD: COMPUTATIONAL ANALYSIS AND CHARACTERIZATION OF VAD V3 .......................................97

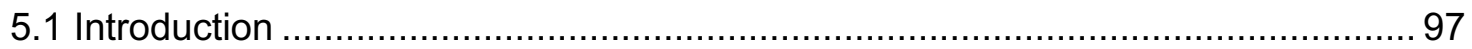

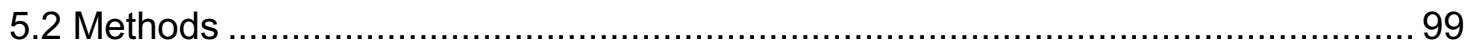

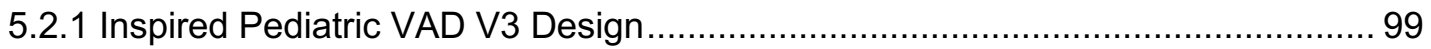

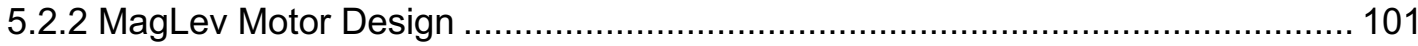

5.2.3 Computational Fluid Dynamics (CFD) Simulation ........................................ 103

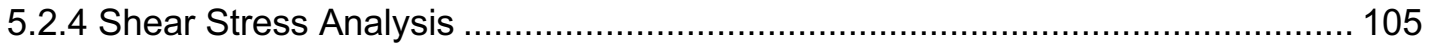

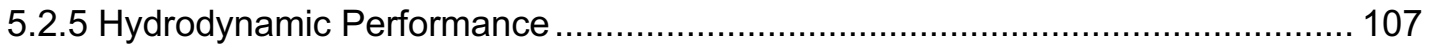

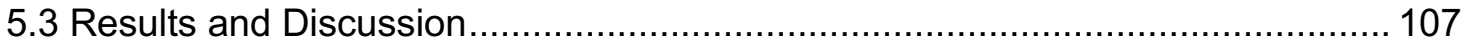

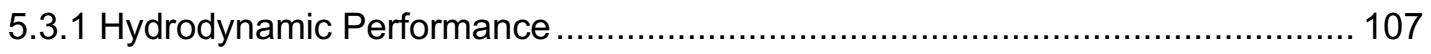

5.3.2 Impeller Torque and Translational Forces...................................................... 108

5.3.3 Pressure Distribution and Flow Fields ....................................................... 111

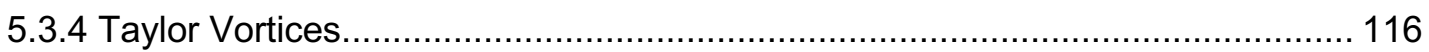

5.3.5 Shear Stress and Residence Time ............................................................. 118

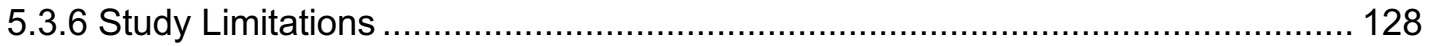

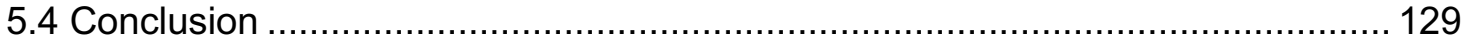

CHAPTER 6 : CONCLUSIONS AND FUTURE WORK ..................................130

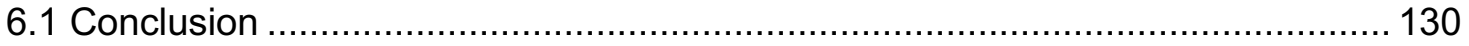

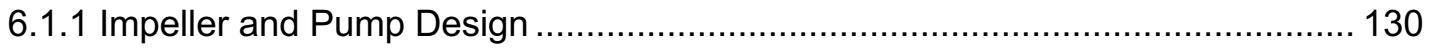

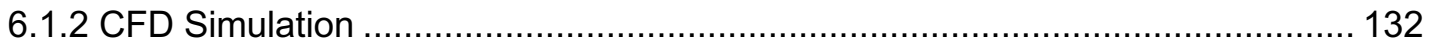

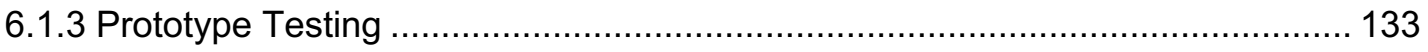

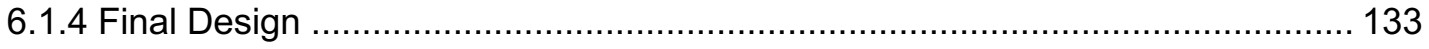

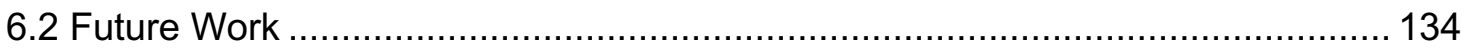

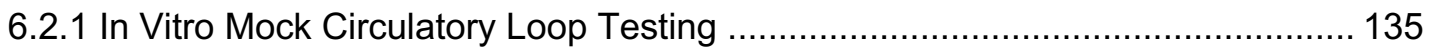

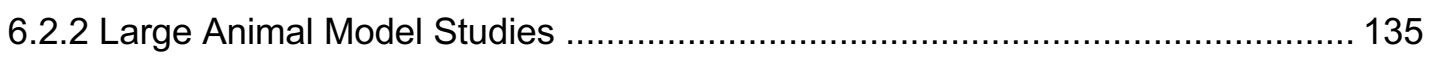

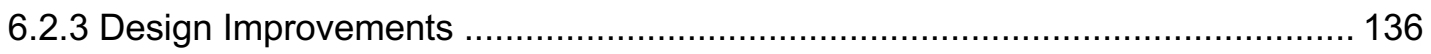

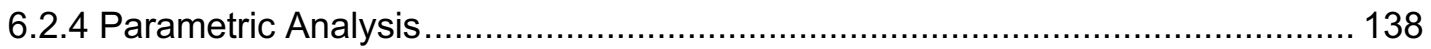

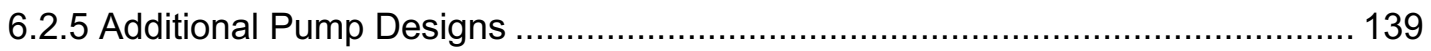

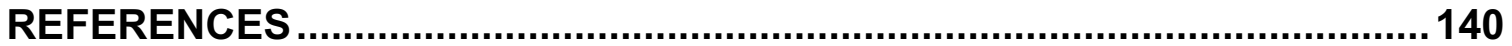


APPENDIX A : Additional Design Equations ….......................................... 150

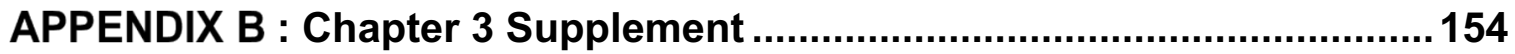

APPENDIX C : Chapter 4 Supplement ........................................................... 167

APPENDIX D : VAD V2 Additional Simulations .......................................... 181

APPENDIX E : Chapter 5 Supplement ......................................................... 187

APPENDIX F : VAD V3 Additional Simulations..........................................199

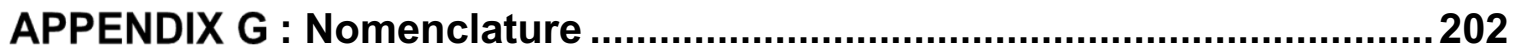

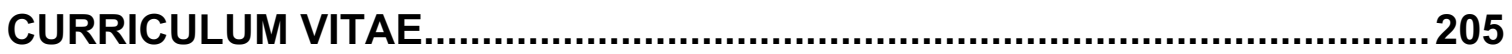




\section{LIST OF TABLES}

$\underline{\text { Page }}$

Table 1-1. Summary of feature goals for the Inspired Pediatric VAD and Universal MagLev System.

Table 2-1. Example of design parameters required for the development of the Inspired Pediatric VAD, including maximum head and flow rate and impeller outer diameter as well as fluid parameters for whole blood. 14

Table 2-2. All calculated parameter values for the entrance and discharge Euler velocity triangles under ideal flow conditions for the Inspired Pediatric VAD impeller.

Table 2-3. All calculated parameter values for the modified entrance and discharge Euler velocity triangles accounting for flow pre-rotation and slip for the Inspired Pediatric VAD impeller........25

Table 2-4. All critical calculated impeller dimensions for the Inspired Pediatric VAD.

Table 3-1. Summary of design features of the Inspired Pediatric VAD compared to current commercially available pediatric extracorporeal VADs: Berlin Heart EXCOR, Medtronic BioPump BP-50, Maquet RotaFlow, Medos Deltastream DP3 and Thoratec PediMag.

Table 3-3. Summary of proposed Inspired Pediatric VAD operational and design criteria.

Table 3-4. All critical calculated impeller dimensions for Pump V1.

Table 3-5. Summary of converged values pressure $(\Delta P)$ and torque and calculated hydraulic efficiency $\left(\eta_{H}\right)$ for Pumps V1 and $\mathrm{V} 2$ at $\mathrm{Q}=3.5 \mathrm{~L} / \mathrm{min}$ and $\mathrm{n}=4255 \mathrm{RPM}$.

Table 4-1. Empirical results for the Inspired Pediatric VAD prototype in the SFL including generated pressure head $(\Delta P)$ and impeller/rotor hydraulic torque $\left(\boldsymbol{\tau}_{\text {Hydro }}\right)$ for selected rotational speed and flow cases (3000, 4000, and 5000 RPM). $U$ = 
Unachievable, meaning the flow rate was not achievable at the given rotational speed by the prototype pump but was achievable in the CFD simulations.

Table 4-2. $\quad$ CFD results for simulation Setup 1 including generated pressure head $(\Delta P)$ and impeller/rotor hydraulic torque $\left(\boldsymbol{\tau}_{\text {Hydro }}\right)$ for selected rotational speed and flow cases (3000, 4000 , and 5000 RPM) and translational force values for the impeller/rotor in the $\mathrm{x}, \mathrm{y}$, and $\mathrm{z}$ directions.

Table 4-3. CFD results for simulation Setup 2 including generated pressure head $(\Delta P)$ and impeller/rotor hydraulic torque $\left(\boldsymbol{\tau}_{\text {Hydro }}\right)$ for selected rotational speed and flow cases (3000, 4000 , and 5000 RPM) and translational force values for the impeller/rotor in the $\mathrm{x}, \mathrm{y}$, and $\mathrm{z}$ directions

Table B-1. Summary of Reynolds number calculations for pump inlet and impellers for Pumps V1 and V2 at $\mathrm{Q}=3.5 \mathrm{~L} / \mathrm{min}$ and various rotational speeds $(\mathrm{n})$.

Table B-2. Four meshing schemes with increasing mesh refinement compared to respective number of mesh cells and converged values for $\triangle \mathrm{P}$ and Torque for both Pump V1 and Pump V2 at $\mathrm{Q}=3.5 \mathrm{~L} / \mathrm{min}$ and $\mathrm{n}=4255 \mathrm{RPM}$.

Table B-3. Calculated simulation time steps based on two recommended methods for various impeller rotational speeds.

Table B-4. Mean wall shear stress (WSS) values for all surfaces on the pump impeller and pump housing for both versions Pump $\mathrm{V} 1$ and V2 at rotational speeds 4255 to 6000 RPM.

Table C-1. DC motor back electromotive force (EMF) at corresponding rotational speeds, generated voltage, calculated back EMF constant, $\mathrm{K}_{\mathrm{e}}$, and motor torque constant, $\mathrm{K}_{\mathrm{t}}$

Table C-2. $\quad$ DC motor voltage $(\mathrm{V})$ and current $(\mathrm{A})$ at multiple rotational speeds against no load. Motor power (W) was calculated from measured voltage, current. Motor torque $\left(\boldsymbol{\tau}_{\mathrm{DC}}\right)$ was calculated from measured current and the derived motor torque constant, $\mathrm{K}_{\mathrm{t}}$

Table C-3. $\quad$ DC motor voltage $(\mathrm{V})$ and current $(\mathrm{A})$ at multiple rotational speeds while driving the Pediatric VAD impeller without fluid. The total torque of the VAD without fluid ( $\boldsymbol{\tau}_{\text {Total, Dry }}$ ) was 
calculated from measured current and $\mathrm{K}_{\mathrm{t}}$. The torque due to friction $\left(\boldsymbol{\tau}_{\text {Friction }}\right)$ of the shaft and seal was determined as the difference between the total torque of the dry system ( $\boldsymbol{\tau}_{\text {Total, }}$ Dry $)$ and the total torque of the DC motor $\left(\boldsymbol{\tau}_{\mathrm{DC}}\right) \ldots$ 168

Table C-4. Collected parameters of the Inspired Pediatric VAD in the Static Flow Loop (SFL) at 500 RPM. Total torque of the VAD in the SFL ( $\boldsymbol{\tau}_{\text {Total }}$ ) was calculated from measured current and $\mathrm{K}_{\mathrm{t}}$. The hydraulic torque ( $\left.\boldsymbol{\tau}_{\text {Hydro }}\right)$ of the impeller was the resulting net torque between the calculated total torque ( $\left.\boldsymbol{\tau}_{\text {Total }}\right)$ of the fluid filled system, the total base torque of the DC motor $\left(\tau_{\mathrm{DC}}\right)$, and the torque due to friction of the shaft and seal $\left(\boldsymbol{\tau}_{\text {Friction }}\right)$. This was done for all rotational speeds $(500-6000$ RPM $)$.

Table C-5. Collected parameters of the Inspired Pediatric VAD in the Static Flow Loop (SFL) at 1000 RPM.

Table C-6. Collected parameters of the Inspired Pediatric VAD in the Static Flow Loop (SFL) at 1500 RPM.

Table C-7. Collected parameters of the Inspired Pediatric VAD in the Static Flow Loop (SFL) at 2000 RPM.

Table C-8. Collected parameters of the Inspired Pediatric VAD in the Static Flow Loop (SFL) at 2500 RPM.

Table C-9. Collected parameters of the Inspired Pediatric VAD in the Static Flow Loop (SFL) at 3000 RPM.

Table C-10. Collected parameters of the Inspired Pediatric VAD in the Static Flow Loop (SFL) at 3500 RPM.

Table C-11. Collected parameters of the Inspired Pediatric VAD in the Static Flow Loop (SFL) at 4000 RPM.

Table C-12. Collected parameters of the Inspired Pediatric VAD in the Static Flow Loop (SFL) at 4500 RPM.

Table C-13. Collected parameters of the Inspired Pediatric VAD in the Static Flow Loop (SFL) at 5000 RPM.

Table C-14. Collected parameters of the Inspired Pediatric VAD in the Static Flow Loop (SFL) at 5500 RPM.

Table C-15. Collected parameters of the Inspired Pediatric VAD in the Static Flow Loop (SFL) at 6000 RPM. 
Table D-1. Empirical results for the Inspired Pediatric VAD prototype in the SFL (EXP1) compared to CFD results for the two different impeller/rotor geometries (CFD1 and CFD2) including generated pressure head $(\Delta \mathrm{P})$ and impeller/rotor hydraulic torque ( $\left.\boldsymbol{\tau}_{\mathrm{Hydr}}\right)$.

Table E-1. Summary of Reynolds number calculations for Inspired Pediatric VAD V3 pump inlet and impeller for flow rates $(\mathrm{Q})$ $0.50-4.00 \mathrm{~L} / \mathrm{min}$ and rotational speeds (n) 3000,4000 , and 5000 RPM.

Table E-2. Summary of computational fluid dynamics (CFD) predictions of head pressure, impeller hydraulic torque, and impeller translational forces for Inspired Pediatric VAD V2 at rotational speeds 3000,4000 , and 5000 RPM and various flow rates.

Table E-3. Summary of computational fluid dynamics (CFD) predictions of head pressure, impeller hydraulic torque, and impeller translational forces for Inspired Pediatric VAD V3 at rotational speeds 3000,4000 , and 5000 RPM and various flow rates.

Table E-4. Summary of data for additional CFD simulation cases for Inspired Pediatric VAD V3 at rotational speeds 1000, 2000, and 6000 RPM.

Table F-1. Computationally predicted hydrodynamic values at 5000 RPM for CFD simulation cases: laminar flow, nonNewtonian fluid; laminar flow, Newtonian fluid; turbulent flow, Newtonian fluid. 200 


\section{LIST OF FIGURES}

Page

Figure 1-1. Flow of the Inspired Pediatric VAD development project with dissertation chapter areas highlighted. 8

Figure 2-1. Rendering of the Inspired Universal MagLev System and Pediatric VAD single-use pump concepts. The dimensions for the system are 5 in. by 2.6 in. (the size of a $7.5 \mathrm{oz}$ container) making it a small and compact device to allow for potential patient ambulation during treatment.

Figure 2-2. Modified Cordier diagram detailing relationships between diameter number, $\delta$, speed number, $\sigma$, and pump types radial, diagonal, and axial. Reproduced from [27] with permission.

Figure 2-3. (A) Depiction of a rotating impeller with fluid flow through the blades and the locations of entrance (red) and discharge (blue) velocity triangles, (B) Entrance velocity triangle for the entrance of the impeller blade, (C) Discharge velocity triangle for the exit of the impeller blade, (D) Entrance velocity triangle accounting for flow recirculation and flow pre-rotation, (E) Discharge velocity triangle accounting for flow recirculation and slip.

Figure 2-4. Impeller design constants $\mathrm{K}_{\mathrm{m} 1}$ and $\mathrm{K}_{\mathrm{m} 2}$ versus pump specific speed $\eta_{\mathrm{s}}$. Reproduced from [24] with permission.........................20

Figure 2-5. Head coefficient $\Psi$ and flow coefficient $\phi$ versus pump specific speed $\eta_{\mathrm{s}}$ for various blade exit angles $\beta_{2}$. Reproduced from [24] with permission.

Figure 2-6. Impeller design constants $\mathrm{K}_{\mathrm{m} 1}$ and $\mathrm{K}_{\mathrm{m} 2}$ versus pump specific speed $\eta_{\mathrm{s}}$. Reproduced from [24] with permission.

Figure 2-7. (A) Representation of the mean blade line plotted using the simple-arc method (SAM), reproduced from [33] with permission, (B) Plot of the mean blade line for the Inspired Pediatric VAD impeller, (C) Plot of all six mean blade lines for the Inspired Pediatric VAD impeller with dimensioned 
entrance and exit angles, (D) Three-dimensional solid model of the basic Inspired Pediatric VAD impeller features.

Figure 2-8. (A) Detailed view of impeller blade shape, thickness, and tip profiling, (B) Rendering of the final Inspired Pediatric VAD impeller concept with details including the impeller shroud, impeller blades, rotor body, rotor magnetics, and rotor body center opening.

Figure 3-1. Components of the Thoratec CentriMag/PediMag system (pump, motor, controller) (Top) (with dimensions shown), compared to the Inspired Pediatric VAD and Universal MagLev System (Bottom) (with goal dimensions shown). The Inspired Universal System combines all extracorporeal components into one small, universal package allowing for potential greater ease of use, quality of care, and possible patient ambulation.

Figure 3-2. Basic components of the Inspired Universal MagLev system and removable Pediatric VAD pump head.

Figure 3-3. Cross section views of the 3D geometry of Pump V1 (V1 A) compared to Pump V2 (V2 A). View of the 3D geometry of the impeller for Pump V1 (V1 B) and Pump V2 (V2 B), looking from the bottom upward to see detail of the blades beneath the shroud. Highlighted impeller and pump features include the shroud, inlet eye, blades and Pump V2 added features blade tip extensions and center post.

Figure 3-4. For $\mathrm{Q}=3.5 \mathrm{~L} / \mathrm{min}$ and $\mathrm{n}=4255 \mathrm{RPM},(\mathrm{V} 1 \mathrm{~A})$ Pressure distribution planar to inlet center of V1, (V1 B) Pressure distribution through impeller blades of V1, (V1 C) Isometric view of pressure distribution at outer housing surfaces of V1, (V2 A) Pressure distribution planar to inlet center of V2, (V2 B) Pressure distribution through impeller blades of V2, (V2 C) Isometric view of pressure distribution at outer housing surfaces of $\mathrm{V} 2$.

Figure 3-5. For $\mathrm{Q}=3.5 \mathrm{~L} / \mathrm{min}$ and $\mathrm{n}=4255 \mathrm{RPM}$, (V1 A) Velocity flow fields at a planar surface cut through the center of the impeller blades for Pump V1, (V2 A) velocity flow fields at a planar surface cut through the center of the impeller blades extensions for Pump V2, (V1 B) three dimensional velocity flow fields planar to inlet center for Pump V1 with highlighted flow paths; primary (white arrows), rotor secondary (grey arrows), pump housing secondary (purple 
arrows), (V2 B) three dimensional velocity flow fields planar to inlet center for Pump V2.

Figure 3-6. Surface plots of Pump V1 and V2 pump housings and impellers indicating areas where wall shear stress (WSS) values exceed $150 \mathrm{~Pa}$ at $\mathrm{Q}=3.5 \mathrm{~L} / \mathrm{min}$ and $\mathrm{n}=4255 \mathrm{RPM}$.

Figure 3-7. $\quad(A)$ Plotted generated pressure $(\triangle P)$ versus rotational speed (n) at $Q=3.5 \mathrm{~L} / \mathrm{min}$ for Pumps V1 and V2, (B) Plotted calculated hydraulic efficiencies $\left(\eta_{H}\right)$ versus rotational speed (n) at $\mathrm{Q}=3.5 \mathrm{~L} / \mathrm{min}$ for Pumps V1 and V2

Figure 3-8. Pressure distributions at a planar surface cut through the middle of the impeller blade extensions for Pump V2 at $\mathrm{Q}=$ $3.5 \mathrm{~L} / \mathrm{min}$ and $(\mathrm{A}) \mathrm{n}=4500 \mathrm{RPM},(\mathrm{B}) \mathrm{n}=5000 \mathrm{RPM},(\mathrm{C}) \mathrm{n}=$ 5500 RPM, (D) $n=6000$ RPM. 56

Figure 4-1. Exploded view of the Inspired Pediatric VAD concept detailing the fluid flow direction, impeller rotational direction (Z-axis), impeller translational force directions (X, Y, Z-axis) and the pump components; the Upper Pump Housing, Impeller, Motor Rotor, Lower Pump Housing, and Motor Stator.

Figure 4-2. Cut-view of the Inspired Pediatric VAD concept detailing the blood/fluid gap between the impeller/rotor and pump housing ( $0.5 \mathrm{~mm}$ along sides, $0.75 \mathrm{~mm}$ along bottom) and the overall gap $(2 \mathrm{~mm})$ between rotor and stator magnetic components.

Figure 4-3. (A) Side and (B) Top views of the prototype of the Inspired Pediatric VAD including SLA polymer impeller, lower pump housing, and $8 \mathrm{~mm}$ metal shaft. (C) VAD prototype attached to custom fixture with coupled DC motor and tachometer, which are connected to the (D) static flow loop (SFL) for preliminary performance testing and evaluation.

Figure 4-4. (A) Computational fluid volumes for CFD simulation Setup 1 and 2, (B) CFD Setup 1, Front and Top plane cut-plots of flow velocity for 5000 RPM at $3.00 \mathrm{~L} / \mathrm{min}$, (C) CFD Setup 2, Front and Top plane cut-plots of flow velocity for 5000 RPM at $3.00 \mathrm{~L} / \mathrm{min}$

Figure 4-5. A flow chart detailing the SFL (using the empirical pump prototype, EXP) and CFD (using Setup 1 and 2) experiments, the tested pump conditions (rotational speed or flow rate and rotational speed) for each experiment, and 
the collected variables for each experiment (pump pressure, flow rate, impeller torque and impeller $x, y$, and $z$ forces). 76

Figure 4-6. Characteristic HQ curves (pressure versus flow rate) for the Inspired Pediatric VAD pump empirical prototype for rotational speeds 500 to 6000 RPM.

Figure 4-7. Hydraulic torque of the Inspired Pediatric VAD empirical prototype impeller/rotor versus flow rate for rotational speeds 500 to 6000 RPM.

Figure 4-9. Hydraulic torque of the VAD empirical prototype versus generated curves from CFD simulations Setups 1 and 2 for rotational speeds 3000,4000 , and 5000 RPM.

Figure 4-8. $\quad \mathrm{HQ}$ curves of the VAD empirical prototype versus generated curves from CFD simulations Setups 1 and 2 for rotational speeds 3000,4000 , and 5000 RPM.

Figure 4-10. Plots of translational $\mathrm{X}, \mathrm{Y}$, and Z-axis forces of the Inspired Pediatric VAD impeller/rotor generated from CFD Setups 1 and 2 at 3000, 4000, and 5000 RPM.

Figure 5-1. Exploded view of the Inspired Pediatric VAD V3 (left) identifying the rotating body (impeller, rotor body, and rotor magnetics) and the stationary body (upper and lower pump housing), and conceptual rendering of the complete Inspired Universal MagLev System with attached Inspired Pediatric VAD (right) identifying the motor housing, universal controller module with battery hook-ups, and the VAD inlet holder cap, which holds the impeller/rotor in a stationary position for transport. 98

Figure 5-2. Comparison of the Inspired pediatric VAD V2 (top left) and V3 (bottom right) illustrating differences in rotor body and pump housing design, which resulted in an increase in priming volume from 9.1 to $14.5 \mathrm{ml}$, and removal of the center post (V3). 100

Figure 5-3. Cross-section view of the MagLev motor design for $\mathrm{V} 3$ showing rotor and stator components and identifying the passive magnetic bearings, voice coil actuator and brushless permanent magnet motor.

Figure 5-4. Computationally predicted characteristic pressure-flow curves $(\mathrm{H}-\mathrm{Q})$ for Inspired VAD V2 and V3 at various rotational speeds (3000, 4000, $5000 \mathrm{RPM})$ and flow rates (0.50 - $4.00 \mathrm{~L} / \mathrm{min})$. 108 
Figure 5-5. Computationally predicted impeller/rotor hydraulic torque values for Inspired VAD V2 and V3 at various rotational speeds $(3000,4000,5000$ RPM $)$ and flow rates $(0.50-4.00$ L/min).

Figure 5-6. Plots of computationally predicted translational $X, Y$, and Zaxis forces of the Inspired Pediatric VAD V2 and V3 impeller/rotor at various rotational speeds $(3000,4000$, $5000 \mathrm{RPM})$ and flow rates $(0.50-4.00 \mathrm{~L} / \mathrm{min})$.

Figure 5-7. Pressure distribution through the impeller blades (top) and planar to pump inlet center (bottom) of the Inspired Pediatric VAD V3 at various rotational speeds (3000, 4000, 5000 $\mathrm{RPM})$ and flow rates $(0.50,1.00,3.50 \mathrm{~L} / \mathrm{min})$.

Figure 5-8. Isometric views of pressure distributions at the pump housing surface of Inspired Pediatric VAD V3 at various rotational speeds (3000, 4000, 5000 RPM) and flow rates $(0.50,1.00,3.50 \mathrm{~L} / \mathrm{min})$.

Figure 5-9. Three-dimensional velocity flow field plots at a planar cut view through the center of the pump inlet of the Inspired Pediatric VAD V3 for rotational speeds 3000,4000 , and $5000 \mathrm{RPM}$ at $0.50,1.00$, and $3.50 \mathrm{~L} / \mathrm{min}$. Detailed views show the flow fields at the pump inlet to volute transition, exit of the impeller blades, and through the rotor body center opening.

Figure 5-10. Velocity plots of the impeller/rotor surface and surrounding flow in a cut plot planar to the pump inlet center opening with black iso-flow lines displayed to show detail of Taylor vortices formation at various flow rates $(0.50$ to $3.50 \mathrm{~L} / \mathrm{min})$ and rotational speeds (3000, 4000, 5000 RPM).

Figure 5-11. Shear rate distribution plots in the flow domain at a planar cut view through the center of the impeller inlet of the Inspired Pediatric VAD V3 at rotational speeds 3000,4000 , and 5000 RPM and flow rates $0.50,1.00,2.00$, and 3.50 $\mathrm{L} / \mathrm{min}$. Detailed views show the shear rate distribution at the blood gap region between the rotor body and lower pump housing surfaces.

Figure 5-12. Shear rate distribution plots in the flow domain at a planar cut view through the center of the impeller blades and through the center of the rotor body of the Inspired Pediatric VAD V3 at rotational speeds 3000,4000 , and 5000 RPM and flow rates $0.50,1.00,2.00$, and $3.50 \mathrm{~L} / \mathrm{min}$. 
Figure 5-13. Wall shear stress distribution plots of the impeller/rotor and pump housing surfaces of the Inspired Pediatric VAD V3 at rotational speeds 3000,4000 , and 5000 RPM and flow rates $0.50,1.00,2.00$, and $3.50 \mathrm{~L} / \mathrm{min}$.

Figure 5-14. Histograms of the volumetric distribution of shear stress (Left) and the residence time distribution of shear stress (Right) of the Inspired Pediatric VAD V3 at rotational speeds of 3000,4000 , and 5000 RPM and flow rates of 0.50, 2.00, and $3.50 \mathrm{~L} / \mathrm{min}$

Figure 5-15. Histograms of the volumetric distribution of shear stress (Top) and the residence time distribution of shear stress (Bottom) of the Inspired Pediatric VAD V3 at critical thresholds of $9 \mathrm{~Pa}$ ( $\mathrm{VWF}$ damage), $50 \mathrm{~Pa}$ (platelet activation), and $150 \mathrm{~Pa}$ (hemolysis).

Figure 5-16. Summary of iso-surface plots detailing areas within the fluid volume where shear rates are below $250 \mathrm{~s}^{-1}$ (possible clot formation at pumping surfaces), $100 \mathrm{~s}^{-1}$ (areas where blood behaves as a non-Newtonian fluid), and flow velocities are below $0.20 \mathrm{~m} / \mathrm{s}$ (areas of possible flow stagnation and recirculation), for various rotational speeds and flow rates.

Figure 6-1. Example of dimensionless constants calculations in the Inspired Pump Design Program.

Figure 6-2. Example of volute dimensions calculations in the Inspired Pump Design Program.

Figure 6-3. Injection molded and additive manufacturing components of the Inspired Pediatric VAD V3 prototype currently under development.

Figure B-1. Plotted converged $\Delta \mathrm{P}$ values versus number of mesh cells for four meshing schemes for both Pump V1 and V2 at Q = $3.5 \mathrm{~L} / \mathrm{min}$ and $\mathrm{n}=4255 \mathrm{RPM}$.

Figure B-2. Unstructured Cartesian-based hexahedral meshing schemes for Pump V1 with increasing levels of cell refinement. Mesh Scheme 3 was chosen for simulation. 158

Figure B-3. Enlarged view of velocity flow field plot for Pump V1 at pump housing volute and impeller blade exit $(Q=3.5 \mathrm{~L} / \mathrm{min}$ at 4255 RPM). 
Figure B-4. Enlarged view of velocity flow field plot for Pump V2 at pump housing volute and impeller blade exit $(Q=3.5 \mathrm{~L} / \mathrm{min}$ at 4255 RPM).

Figure B-5. Enlarged view of velocity flow field plot for Pump V1 at the bottom of the pump housing and rotor body center opening $(\mathrm{Q}=3.5 \mathrm{~L} / \mathrm{min}$ at $4255 \mathrm{RPM})$.

Figure B-6. Enlarged view of velocity flow field plot for Pump V2 at the pump housing center post and rotor body center opening $(Q$ $=3.5 \mathrm{~L} / \mathrm{min}$ at $4255 \mathrm{RPM})$.

Figure B-7. Enlarged view of velocity flow field plot for Pump V1 at the pump housing cut-water (split at pump volute and pump outlet) $(\mathrm{Q}=3.5 \mathrm{~L} / \mathrm{min}$ at $4255 \mathrm{RPM})$.

Figure B-8. Enlarged view of velocity flow field plot for Pump V2 at the pump housing cut-water (split at pump volute and pump outlet) $(\mathrm{Q}=3.5 \mathrm{~L} / \mathrm{min}$ at $4255 \mathrm{RPM})$.

Figure B-9. Enlarged view of velocity flow field plot for Pump V1 through the impeller blades and into the pump housing volute $(\mathrm{Q}=$ $3.5 \mathrm{~L} / \mathrm{min}$ at $4255 \mathrm{RPM}$ ). 165

Figure C-1. Plot of calculated DC motor torque against no load versus rotational speed. The linear line of best fit and subsequent linear equation were used to determine the motor damping coefficient $\left(B_{m}\right)$ and the kinetic friction of the motor $\left(T_{f m}\right)$ and to calculate $\mathrm{DC}$ motor torque $\left(\tau_{\mathrm{DC}}\right)$ at any rotational speed. 169

Figure C-2. Calibrated Cannon-Fenske style viscometer submerged in a $37^{\circ} \mathrm{C}$ water bath to measure the viscosity of the blood analogue solution. sample.

Figure C-3. (Left) Laser-based tachometer to measure impeller rotational speed, (Right) PMDC motor and shaft with coupler attached to SLA printed pump impeller.

Figure C-4. Various images of static flow loop (SFL) construction with a view (Bottom Right) of the variable DC power supply, multimeters, and DAQ data collection system

Figure C-5. View of the completed static flow loop system with heated water bath.

Figure D-1. Cut-views detailing the original, Inspired Pediatric VAD V2 geometry with rotor body center opening and lower pump housing center post used for CFD1 and of the modified 
VAD2 geometry without center opening used for CFD2 to mimic the geometry of the Inspired Pediatric VAD V2 shaft driven prototype (EXP1)

Figure D-2. A flow chart detailing the SFL (using the empirical pump prototype, EXP1) and CFD (using the pump geometries CFD1 and CFD2) experiments, the tested pump conditions (rotational speed or flow rate and rotational speed) for each experiment, and the collected variables for each experiment (pump pressure, flow rate, impeller torque and impeller $\mathrm{x}, \mathrm{y}$, and $z$ forces).

Figure D-3. Pressure vs. flow rate values for the Inspired Pediatric VAD V2 prototype compared with CFD simulation results CFD1 and CFD 2 for rotational speeds 500 to 6000 RPM.

Figure D-4. Hydraulic torque values for the Inspired Pediatric VAD V2 prototype compared with CFD simulation results CFD1 and CFD 2 for rotational speeds 500 to 6000 RPM.

Figure D-5. Impeller/rotor force in the axial (Z-axis) direction relative to rotational speed for geometries CFD1 and CFD2.

Figure E-1. Meshing schemes for mesh independence analysis of the Inspired Pediatric VAD V3 with increasing levels of cell refinement.

Figure E-2. Generated pressure $(\Delta \mathrm{P})$ and torque values for meshing schemes $1-4$ at $3000 \mathrm{RPM}$ and $1.00 \mathrm{~L} / \mathrm{min}$. Meshing Scheme 3 showed less than a $0.50 \%$ difference in converged $\Delta P$ value and less than a $1 \%$ difference in converged torque value compared to the finest meshing Scheme 4.

Figure E-3. Cut plots planar to the center of the pump inlet showing velocity distributions at $0.010,0.050,0.100,0.200,0.300$, and 0.500 seconds of physical flow time during the transient CFD simulation for $3000 \mathrm{RPM}$ at $1.00 \mathrm{~L} / \mathrm{min}$ and $5000 \mathrm{RPM}$ at $2.50 \mathrm{~L} / \mathrm{min}$ 192

Figure E-4. Average mesh cell residence times at critical shear stress thresholds of $9 \mathrm{~Pa}$ (vWF damage), $50 \mathrm{~Pa}$ (platelet activation), and $150 \mathrm{~Pa}$ (hemolysis) for flow rates $0.50,1.00$, 2.00 , and $3.50 \mathrm{~L} / \mathrm{min}$

Figure E-5. Velocity flow field vectors at the Inspired Pediatric VAD V3 pump outlet. At low flows of $1.00 \mathrm{~L} / \mathrm{min}$ or lower, select areas of flow recirculation and retrograde occur. 
Figure E-6. Velocity flow field vectors at the Inspired Pediatric VAD V3 pump outlet. At higher flows above $1.00 \mathrm{~L} / \mathrm{min}$, all flow vectors travel toward the pump outlet eliminating stagnation and recirculation zones that are present at lower flows.

Figure E-7. Velocity flow field vectors inside the Inspired Pediatric VAD V3 volute at four flow and pressure conditions.

Figure E-8. Velocity flow field vectors inside the Inspired Pediatric VAD V3 impeller eye at four flow and pressure conditions.

Figure E-9. Velocity flow field vectors at the Inspired Pediatric VAD V3 inlet at four flow and pressure conditions.

Figure F-1. A) Computationally predicted characteristic pressure-flow curves (H-Q) and, B) computationally predicted impeller/rotor hydraulic torque values at 5000 RPM for CFD simulation cases: laminar flow, non-Newtonian fluid; laminar flow, Newtonian fluid; turbulent flow, Newtonian fluid. 


\section{CHAPTER 1 : INTRODUCTION}

\subsection{Significance}

\subsubsection{Adult Heart Failure}

Heart failure (HF) remains the leading cause of death, affecting an estimated 26 million people worldwide [1], [2]. HF affects approximately 6.5 million adults in the United States with a projected increase in prevalence of $46 \%$ by 2030 [3]. In 2012, the direct and indirect costs of HF treatment in the United States was $\$ 31$ billion and is projected to increase to $\$ 70$ billion by 2030 [4]. Globally, the economic burden of HF is estimated at $\$ 108$ billion annually [5]. Heart transplantation continues to be the preferred option for long-term survival with end stage HF patients, but the number of transplant candidates continues to outpace the donor pool each year. In 2018, 2,967 adult heart transplantations were performed in the US, while 3,883 new transplant candidates were added [6]. Currently in 2020, there are 3,487 patients awaiting a heart transplantation in the U.S. [7]. Mechanical circulatory support (MCS) has emerged as a promising alternative therapy for advanced HF patients over the last few decades. MCS devices have been used successfully for treating multiple modalities, including left, right, or bi-ventricular support (LVAD, RVAD, BiVAD), cardiopulmonary support (ECMO), and cardiopulmonary bypass (CPB). 
Extracorporeal MCS systems, such as the Inspired MCS system under development, can be used as a bridge-to-decision (BTD) to stabilize the patient until they either recover, undergo heart transplantation (BTT) or to a longer-term intracorporeal ventricular assist system. Implantable ventricular assist systems may also be used for destination therapy (DT).

\subsubsection{Pediatric Heart Failure}

MCS is perceived as the best option for maintaining growth and development in pediatric HF patients as an alternative to transplantation, but the development of MCS devices specifically for the pediatric population has been greatly outpaced compared to those for adults [8], [9]. Up to 14,000 children are hospitalized with HF-related conditions each year in the U.S., with an overall mortality of $7 \%$ [10]. In 2018, 685 new pediatric transplantation candidates were added to the waiting list with only 473 transplants performed [6]. Currently, $17 \%$ of pediatric patients listed for HT die each year while waiting for a donor organ, which is the highest wait-list mortality rate of any organ in the U.S. [11].

\subsubsection{Pediatric Ventricular Support}

Several attempts have been made to develop MCS devices for pediatric HF patients since the National Heart, Lung, and Blood Institute (NHLBI) initiated the Pediatric Circulatory Support Program and Pumps for Kids, Infants, and Neonates (PumpKIN) trial in 2004. NHLBI provided over $\$ 23$ million in development funding to support the PumpKIN program [12]. Of the many devices 
funded in this effort, the Jarvik 2015 VAD is the only one still actively being developed [13]. Fully implantable devices such as the Jarvik 2015 have raised concern among the pediatric clinical community due to invasiveness of surgical procedure, post-op recovery, wide range of patient size, and patient growth during treatment [14]. Alternatively, extracorporeal devices present an excellent option for continued pediatric patient support through growth and development. Unfortunately, the majority of extracorporeal devices available to pediatric patients are intended for adult use [15].

\subsubsection{Limitations of Current Technology}

There are many MCS devices available for the adult HF population for a variety of different indications for use, each with their own unique technology, modes of operation, and user interfaces. Keeping up with training and knowledge of the varying devices can be daunting for patients, caregivers, and clinicians, which may contribute to the risk of human errors with post-op management and long-term support. At present, there are limited MCS options specific to the pediatric HF population. Adding to the complexity of currently available adult MCS systems is the economic burden with mean costs of $\$ 175,420$ for LVAD implantation and $\$ 90,147$ for pump replacement [16]. Additionally, the majority of clinically available extracorporeal systems are large, bulky, bedside units and many use permanent bearings for pump impeller rotation, which can lead to clinically significant blood damage and early device failure [17], [18]. 


\subsubsection{Inspired Pediatric VAD and Universal MagLev System}

The Inspired Universal MagLev system is a new extracorporeal MCS therapeutic option aimed at addressing each of the limitations associated with current devices. The Inspired system features a single motor/controller unit that will utilize interchangeable magnetically levitated (MagLev) single-use pumps for treatment of multiple pediatric and adult cardiopulmonary indications. The first single-use pump developed for the system is intended for pediatric left, right, and biventricular support (Inspired Pediatric VAD), which is the focus of this dissertation. This new system intends to improve upon current MCS offerings by:

\section{Creating a Universal System}

Developing a single integrated pump/motor/controller system with a simple user interface and control scheme that can be used for multiple patient populations (neonate, pediatric, adult) and indications (circulatory support, ECMO, CPB) that will streamline device training and ideally mitigate clinician and caretaker errors.

\section{Creating Modular Pumps for Multiple Indications}

A universal integrated motor/controller designed to drive single-use adult and pediatric ventricular and respiratory assist pumps for up to 30 days of support, and thereby potentially reducing development times and costs (one motor/controller technology, multiple pump impellers). 


\section{Miniaturization}

Miniaturization of electronic and magnetic motor components and integration of multiple peripherals into a single functional unit allows for the creation of a universal system with less complexity, lower cost, and may allow for patient ambulation during treatment.

\section{Improving Durability}

Utilization of MagLev technology to levitate and rotate the impeller/rotor within the pump housing eliminates pump parts from coming in contact with the blood stream, which may lead to improved device hemocompatibility, durability, and longevity.

\section{Reduced Cost}

Combining individual components into a single universal system and utilizing lower cost materials (polycarbonate) to manufacture single-use pump components reduces the cost of the device. Additionally, hospitals will be able to purchase and train clinical caretakers on a single system that supports multiple clinical applications rather than needing to purchase and train staff on multiple more expensive devices, which may lead to significant cost savings with the use of the Inspired System. Additional advantages of the Inspired System are listed in Table 1-1. 
Table 1-1. Summary of feature goals for the Inspired Pediatric VAD and Universal MagLev System.

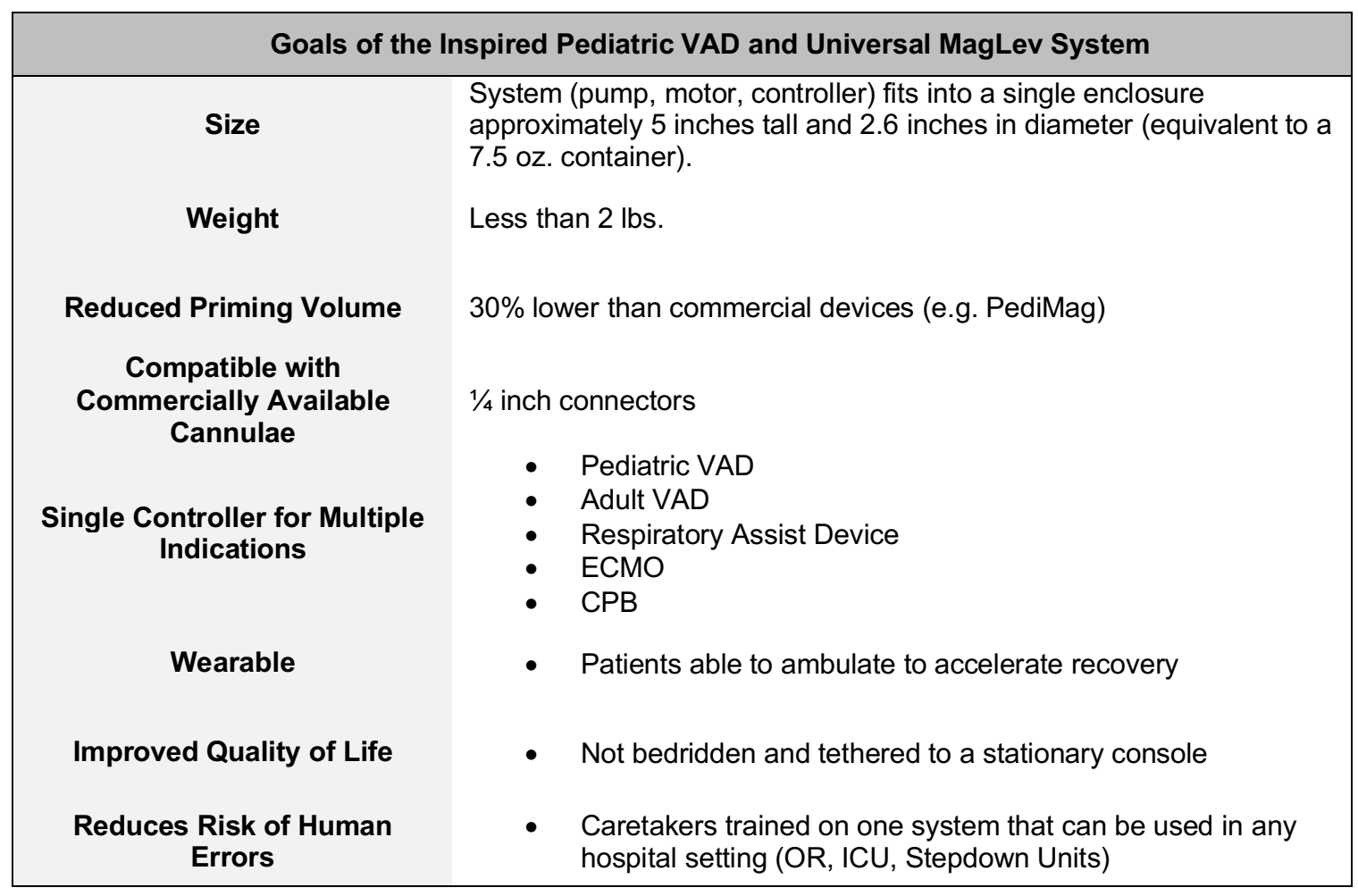

\subsection{Aims and Objectives}

\subsubsection{Aim}

The overall aim for this project was to design, develop, and evaluate the impeller and pump housing components of the Inspired Pediatric VAD through multiple iterations.

\subsubsection{Objectives}

This aim was achieved through the completion of the following project objectives: 
- Define required operational and physiological parameters of the Inspired Pediatric VAD (Chapter 3)

- Design the Inspired Pediatric VAD impeller geometry through calculation and dimensional analysis (Chapter 2)

- Develop a computational fluid dynamics (CFD) model to evaluate the Inspired Pediatric VAD impeller and pump design (Chapter 3)

- Characterize the Inspired Pediatric VAD hydrodynamic performance and impeller torques and forces through CFD prediction (Chapter 3 \& Chapter 4)

- Construct a prototype of the Inspired Pediatric VAD and characterize the hydrodynamic performance and impeller torque in vitro using a static flow loop model (Chapter 4)

- Characterize and evaluate the hydro- and hemodynamics of the final Inspired Pediatric VAD design through CFD prediction (Chapter 5)

\subsection{Overview}

This dissertation is comprised of four core research manuscripts, each presented as individual chapters (Chapters $2-5$ ) that describe the different phases of the Inspired Pediatric VAD development. The four manuscripts have been submitted to scientific journals for peer-review. Figure 1-1 illustrates the flow of the VAD project. The different phases of the project are highlighted to indicate the corresponding chapter within in this dissertation. 


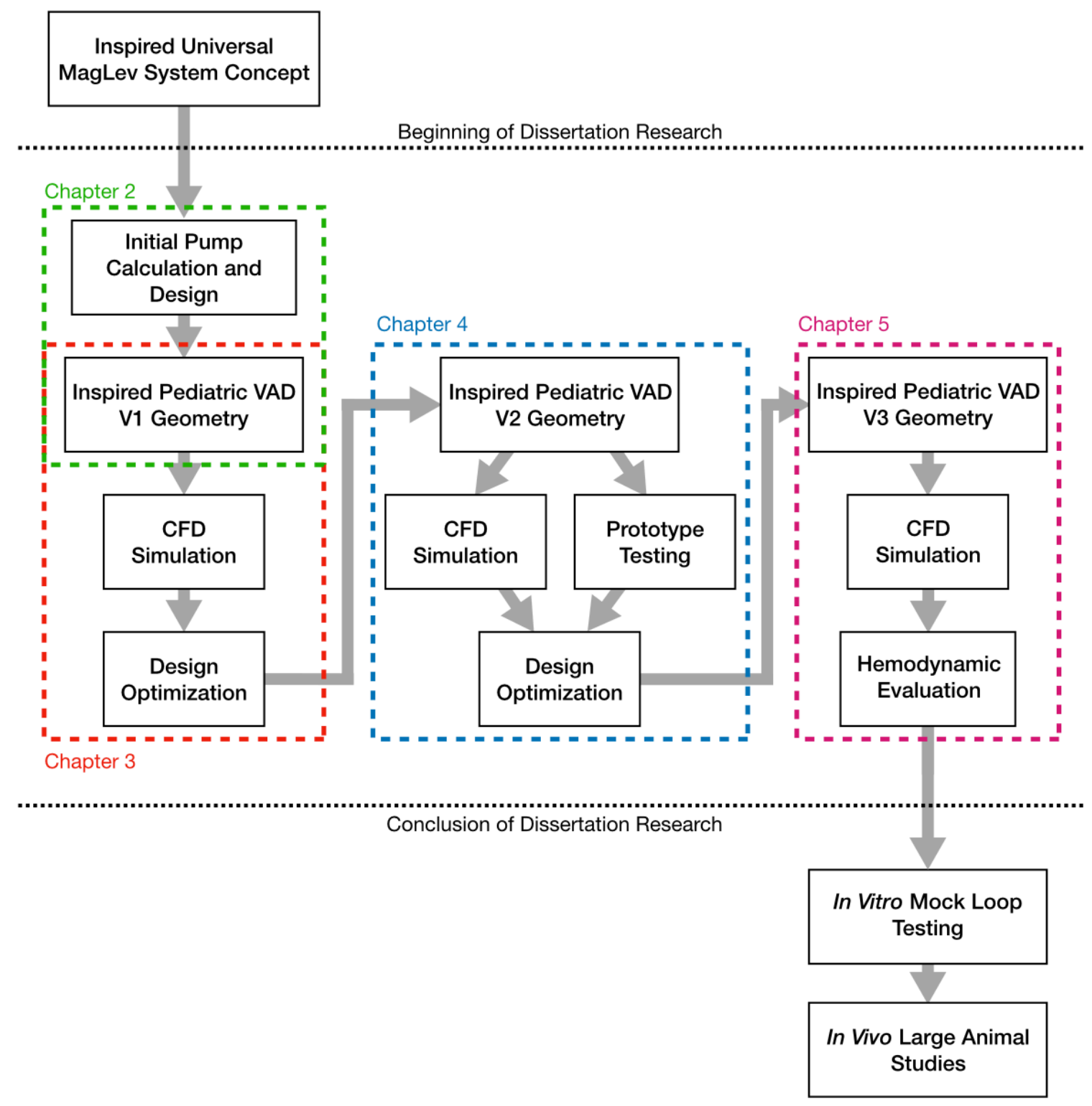

Figure 1-1. Flow of the Inspired Pediatric VAD development project with dissertation chapter areas highlighted.

Chapter 2 provides the calculations and dimensional analysis techniques used to design the first iteration of the VAD impeller (VAD V1) to meet the circulatory requirements of the pediatric population. Chapter 3 describes the construction of a computational fluid dynamics (CFD) model to simulate and evaluate the initial VAD V1 design as well as improved 2 nd generation iterative 
design, VAD V2. Chapter 4 presents CFD simulation studies for the shaft-driven VAD V2 design and mock loop test results, which aided in the design of the novel MagLev motor system. Chapter 5 describes the final iteration of the impeller/pump design (VAD V3) along with the hydrodynamic and hemodynamic evaluation using CFD simulations. 


\section{CHAPTER 2 : IMPELLER DESIGN OF AN EXTRACORPOREAL PEDIATRIC LVAD FOR USE WITH INSPIRED UNIVERSAL MAGLEV SYSTEM}

\subsection{Preface}

Inspired Therapeutics (Merritt Island, FL), in collaboration with the University of Louisville (Louisville, KY), is developing a miniature universal motor/controller to drive single-use extracorporeal pumps and integrated pump/oxygenators in multiple settings (OR, ICU, step-down units) with the objective of minimizing the risk of human errors. Training on a single universal controller for multiple indications for use throughout hospital settings, rather than on a number of different types of controllers, promises to reduce the risk of human errors. Moreover, the controller is miniaturized for use either in a bench top configuration or as a wearable device to allow patients to ambulate earlier in their postoperative recovery period. This article focuses on the first of a series of

\footnotetext{
Authors: ${ }^{1}$ Landon H. Tompkins, MEng., ${ }^{2}$ Barry N. Gellman M.S., ${ }^{1,3}$ Steven C. Koenig Ph.D., ${ }^{1,2,3}$ Kurt A. Dasse Ph.D.

Department of Bioengineering, University of Louisville, Louisville, $K Y$

2 Inspired Therapeutics LLC, Merritt Island, FL

3 Department of Cardiovascular and Thoracic Surgery, University of Louisville, Louisville, KY
} 
pumps and pump/oxygenators to be developed, the Inspired Pediatric VAD, to be used with the Inspired Universal MagLev System (Figure 2-1). The Inspired Pediatric VAD is intended to provide circulatory support for up to 30 -days for

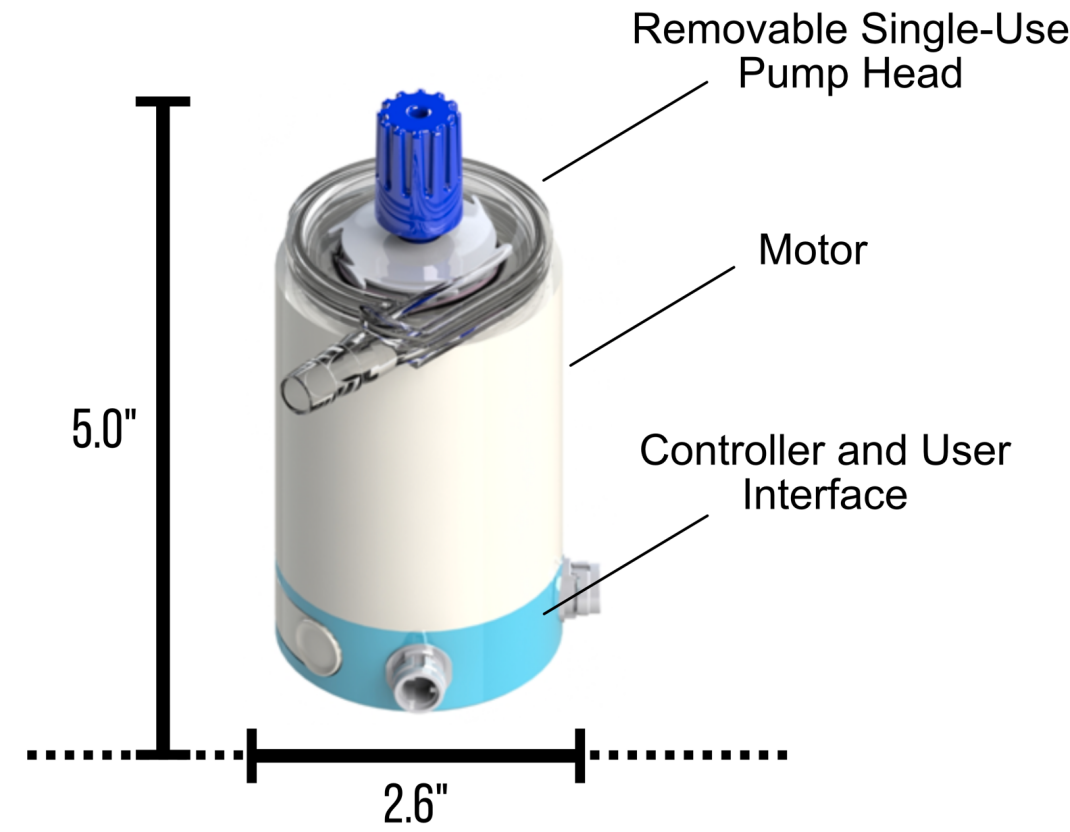

\section{INSPIRED UNIVERSAL MAGLEV SYSTEM}

neonates and pediatric patients.

Figure 2-1. Rendering of the Inspired Universal MagLev System and Pediatric $V A D$ single-use pump concepts. The dimensions for the system are 5 in. by 2.6 in. (the size of a $7.5 \mathrm{oz}$ container) making it a small and compact device to allow for potential patient ambulation during treatment.

In this article, we detail the initial concept formation and preliminary calculations to design the impeller for the first single-use pump head for use with the Inspired Universal MagLev System, the Inspired Pediatric VAD, intended to provide systemic circulatory support for pediatric patients ( $<40 \mathrm{~kg}$ weight) with diseases such as cardiomyopathy, myocarditis, and congenital malformations. 
There are many well established theoretical and experimental approaches and methodologies for the development of traditional turbomachinery that have also been applied to the design of ventricular assist devices (VADs), including centrifugal and axial rotary pumps. Designing an impeller for a rotary blood pump requires balancing the hydrodynamic demands of the pump with its hemocompatibility (hemolysis rate), which is key to clinical success [19]-[21]. Typically, impeller development requires a combination of traditional pump theory calculations, computational fluid dynamics (CFD) simulations, and empirical prototype evaluation, which are used to design and develop a clinically relevant impeller [20]. A first step in this process is to establish operational parameters for the device (pressures, flows) and then apply pump theory to determine the geometries of the impeller body and blades. In this article, traditional pump theory methodologies used to calculate the initial design of the Inspired Pediatric VAD centrifugal pump miniaturized impeller is presented.

\subsection{Impeller Rotational Speed Estimation}

First, the design point, including desired flow rate $(Q)$ and head pressure $(\Delta P)$ at a corresponding rotational speed $(n)$ as well as the diameter (outer) of the impeller $\left(D_{2}\right)$, was defined (Table 2-1) as previously reported [22]. Next, the empirical work of Cordier [23], Stepanoff [24], and Pfleiderer [25] was applied to calculate non-dimensional values and estimate the rotational speed of the impeller at the desired design point as follows: 
Specific work of the pump ( $Y$, Equation 2.1) was calculated to determine the diameter ( $\delta$, Equation 2.2). Using these values, a corresponding speed number of $\sigma=0.125$ was found using a modified Cordier Diagram (Figure 2-2). The estimated rotational speed $(n)$ was then calculated through modification of the speed number formula (Equation 2.3) [19].

$$
\begin{aligned}
& Y=\frac{\Delta P}{\rho}=\frac{0.199 \times 10^{5} \frac{\mathrm{N}}{\mathrm{m}^{2}}}{1060 \frac{\mathrm{kg}}{\mathrm{m}^{3}}}=18.80 \mathrm{~m}^{2} / \mathrm{s}^{2} \\
& \delta=\frac{\sqrt{\pi}}{2} D \sqrt[4]{\frac{2 Y}{Q^{2}}}=0.8862 \times 0.0274 m \times \sqrt[4]{\frac{2 \times 18.80 \frac{m^{2}}{s^{2}}}{\left(5.833 \times 10^{-5} \frac{m^{3}}{s}\right)^{2}}}=7.89 \\
& n=\frac{\sigma}{2.108} \frac{(Y)^{\frac{3}{4}}}{\sqrt{Q}}=\frac{0.125}{2.108} \frac{\left(18.80 \frac{m^{2}}{s^{2}}\right)^{\frac{3}{4}}}{\sqrt{5.833 \times 10^{-5} \frac{m^{3}}{s}}} \times \frac{60 s}{\min }=4254.3 R P M \rightarrow \mathbf{4 2 5 5} \boldsymbol{R P} \boldsymbol{M}
\end{aligned}
$$


Table 2-1. Example of design parameters required for the development of the Inspired Pediatric VAD, including maximum head and flow rate and impeller outer diameter as well as fluid parameters for whole blood.

\begin{tabular}{|c|c|c|c|c|}
\hline \multicolumn{5}{|c|}{ Design Parameters } \\
\hline Parameter & Symbol & & Value & \\
\hline Maximum Pressure Head & $\Delta P$ & $150 \mathrm{mmHg}$ & $0.199 \times 10^{5} \mathrm{~N} / \mathrm{m}^{2}$ & $2.90 \mathrm{psi}$ \\
\hline Maximum Flow Rate & Q & $3.5 \mathrm{~L} / \mathrm{min}$ & $5.833 \times 10^{-5} \mathrm{~m}^{3} / \mathrm{sec}$ & $\begin{array}{c}0.925 \\
\text { Gallon/min }\end{array}$ \\
\hline Impeller Outer Diameter & $\mathrm{D}_{2}$ & $27.4 \mathrm{~mm}$ & $0.0274 \mathrm{~m}$ & - \\
\hline \multicolumn{5}{|c|}{ Natural Parameters } \\
\hline Parameter & \multicolumn{2}{|c|}{ Symbol } & \multicolumn{2}{|c|}{ Value } \\
\hline Blood Density & \multicolumn{2}{|r|}{$\rho$} & \multicolumn{2}{|c|}{$1060 \mathrm{~kg} / \mathrm{m}^{3}$} \\
\hline Blood Viscosity & & $\mu$ & \multicolumn{2}{|c|}{$0.003 \mathrm{~N}^{*} \mathrm{sec} / \mathrm{m}^{2}$} \\
\hline Gravitational acceleration & & g & \multicolumn{2}{|c|}{$9.81 \mathrm{~m} / \mathrm{sec}^{2}$} \\
\hline Specific Gravity of Blood & & SG & \multicolumn{2}{|c|}{$1.0506 @ 37^{\circ} \mathrm{C}$} \\
\hline
\end{tabular}

\subsection{Determination of Pump Specific Speed}

The concept of dimensional analysis was applied to aid in developing a pump design based on maximum efficiency for the required design point [26]. In opposition to the design of industrial pumps, in the design of blood pumps, pump efficiency is typically secondary to the hemocompatibility of the design; however, the Inspired Pediatric VAD was designed for maximum efficiency, but will likely be modified later in the development process to improve hemocompatibility, if needed, at the expense of hydraulic efficiency.

To implement dimensional analysis, the variables describing the pump $(Q$, $\Delta \mathrm{P}, \mathrm{n}, \mathrm{D})$ were reduced to a smaller group of dimensionless numbers to 
characterize the resulting pump design. In this manner, a complete equation involving all dimensionless numbers was created, providing a relationship between all dimensioned variables (known or unknown) defining the pump. Dimensionless variables for pump design include specific speed, $\eta_{\mathrm{s}}$, speed constants, $K_{m 1}$ and $K_{m 2}$, head coefficient, $\psi$, and flow coefficient, $\phi$. Per Stepanoff's [24] method for the dimensionless specific-speed calculation, $\Delta \mathrm{P}$ was converted to feet head $(H)$ to satisfy the units. Pump specific speed $\left(\eta_{s}\right)$ was calculated (Equations 2.4, 2.5).

$$
\begin{gathered}
H=\frac{2.31 \Delta P}{S G}=\frac{2.31 \times 2.90 \mathrm{psi}}{1.0506}=6.38 \text { feet head of blood } \\
\eta_{s}=\frac{n \sqrt{Q}}{H^{\frac{3}{4}}}=\frac{4255 \sqrt{0.925 \mathrm{gpm}}}{6.38 \text { ft. head }} \frac{\frac{3}{4}}{1019.5}
\end{gathered}
$$




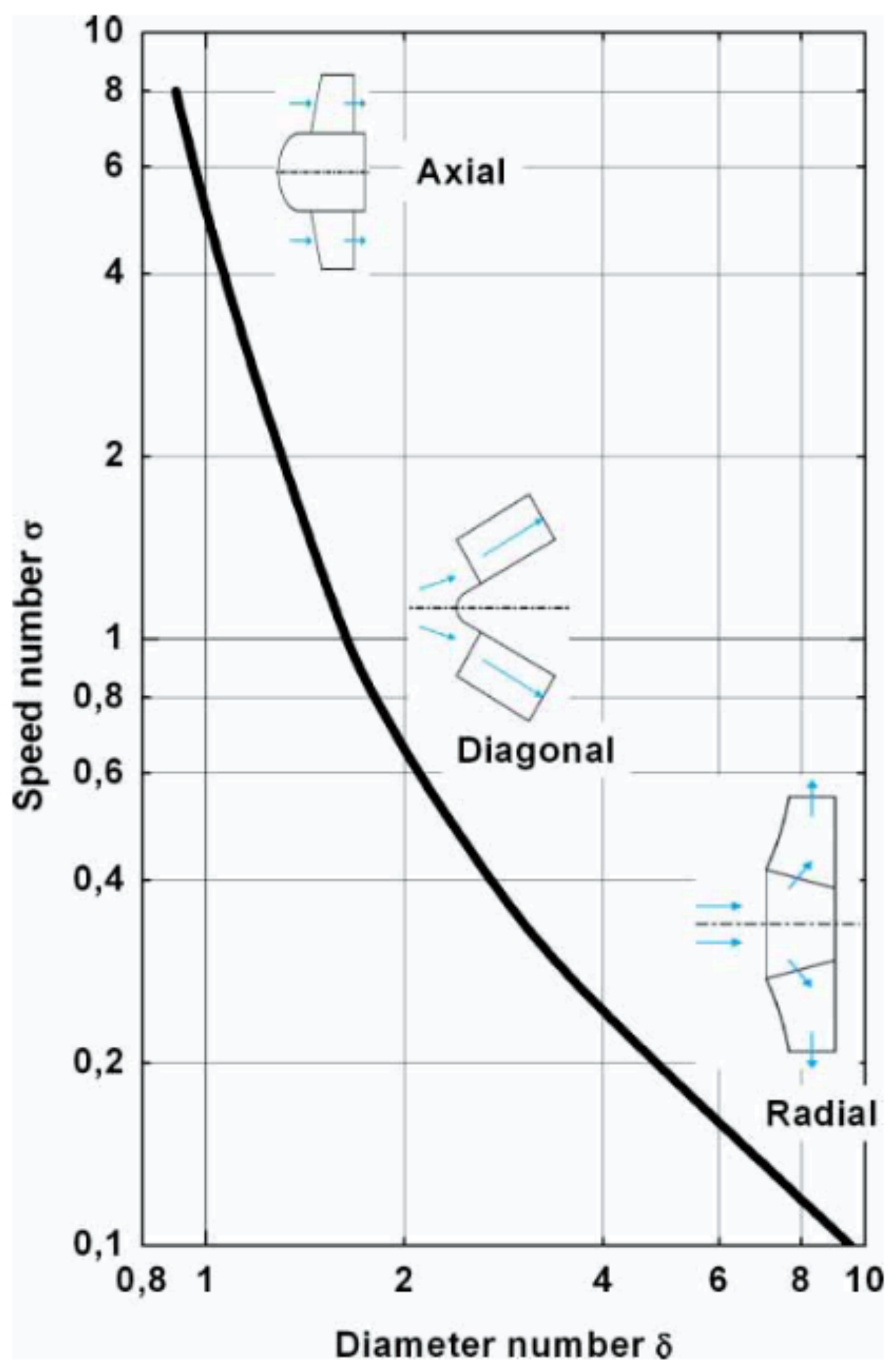

Figure 2-2. Modified Cordier diagram detailing relationships between diameter number, $\delta$, speed number, $\sigma$, and pump types radial, diagonal, and axial. Reproduced from [27] with permission. 


\subsection{Solve Euler Velocity Triangles}

Velocity vectors in one-dimension were estimated for any point along the fluid flow path through a pump impeller (Figure 2-3A). Graphically, the shape of these vectors forms a triangle for each point that is explored. The two most critical points are the entrance and discharge regions of the impeller blades. At these points, the fluid flow can be described by three velocity vectors: (1) relative velocity of the fluid, $\omega$, which is relative to the rotation of the impeller; (2) absolute velocity of the fluid, $c$, which is relative to the pump housing; and (3) peripheral velocity of the impeller, $u$, relative to the center axis. Two velocity triangles were used to characterize the impeller blade: (1) entrance or inlet triangle with subscript 1, and (2) discharge or exit triangle with subscript 2 (Figure 2-3B and C). These velocity vectors are governed by Euler's pump equations and represent one-dimensional flows under ideal conditions (frictionless) [28]. The vectors are further be related by two angles: (1) absolute flow angle, $\alpha$, in relation to the absolute flow velocities; and (2) relative flow angle, $\beta$, in relation to the relative flow velocities, which also corresponds to the angle of the impeller blade at that point.

Following the methods of Stepanoff [24], the Euler velocity triangles were solved. The blade discharge or exit angle, $\beta_{2}$, is critical in determining the impeller design constants (dimensionless numbers). $\beta_{2}$ also directly impacts the shape (steepness) of the pump pressure head and flow relation (HQ curves). Backward swept blades $\left(\beta_{2}<90^{\circ}\right)$ have been shown to induce less blood damage (hemolysis) than other configurations [29]. 


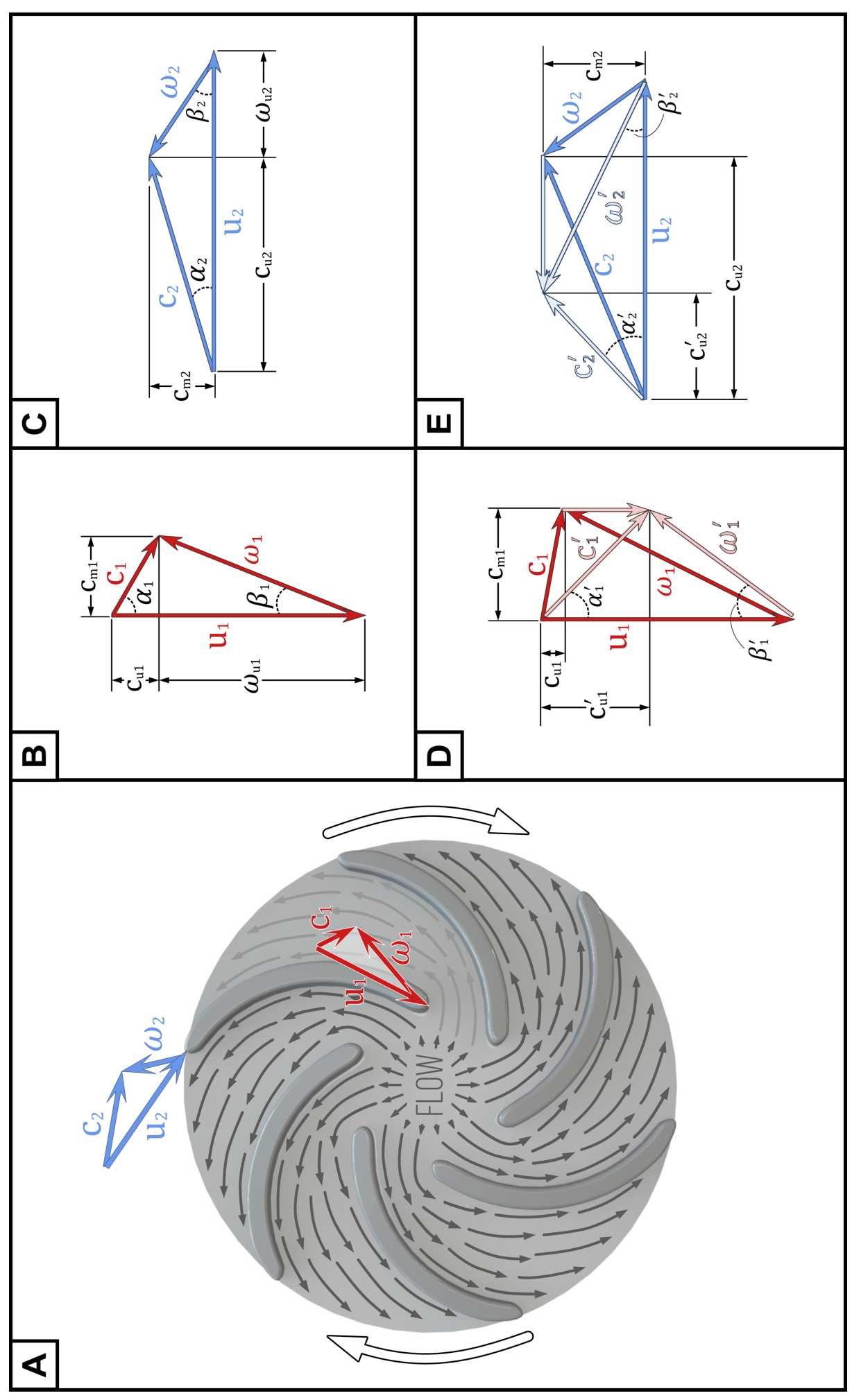

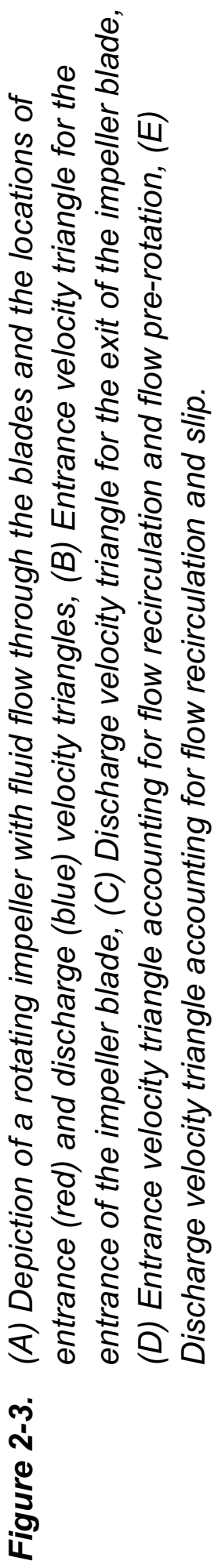


According to Stepanoff [24], for a single-stage centrifugal pump at optimum efficiency, $\beta_{2}$ is between $17.5^{\circ}$ and $27.5^{\circ}$. Additionally, $\beta_{2}$ in the range of $15^{\circ}$ and $30^{\circ}$ has been shown to provide a good balance between impeller efficiency and induced hemolysis [30]. For the Inspired Pediatric VAD, $\beta_{2}=22.5^{\circ}$ was selected for preliminary design as a tradeoff between optimal efficiency and hemocompatibility.

Speed constants are design constants used by Stepanoff [24] and others to relate the pump total head to the impeller peripheral velocity, $U$. Entrance and discharge speed constants, $K_{m 1}$ and $K_{m 2}$, were used to help determine the absolute meridional velocity of the fluid, $C_{m}$. Figure 2-4 illustrates the values used to determine speed constants based on the pump's specific speed, which for our Pediatric VAD resulted in $K_{m 1}=0.195$ and $K_{m 2}=0.138$. Head and flow coefficients serve as design constants representing the head potential (pressure) and capacity potential (flow) of the pump. Figure 2-5 illustrates the values used to determine head and flow constants based on the pump's specific speed, which for our Pediatric VAD resulted in coefficients of $\psi=0.515$ and $\phi=0.115$.

With all design constants determined, the Euler velocity discharge and entrance triangles were solved for ideal flow conditions. Solving the discharge triangle, the discharge blade tip speed, $u_{2}$, as well as the meridional, $C_{m 2}$, tangential, $C_{u 2}$, and total absolute, $C_{2}$, velocities of the fluid were calculated. From these, the absolute fluid discharge angle, $\alpha_{2}$, and the height of the impeller 


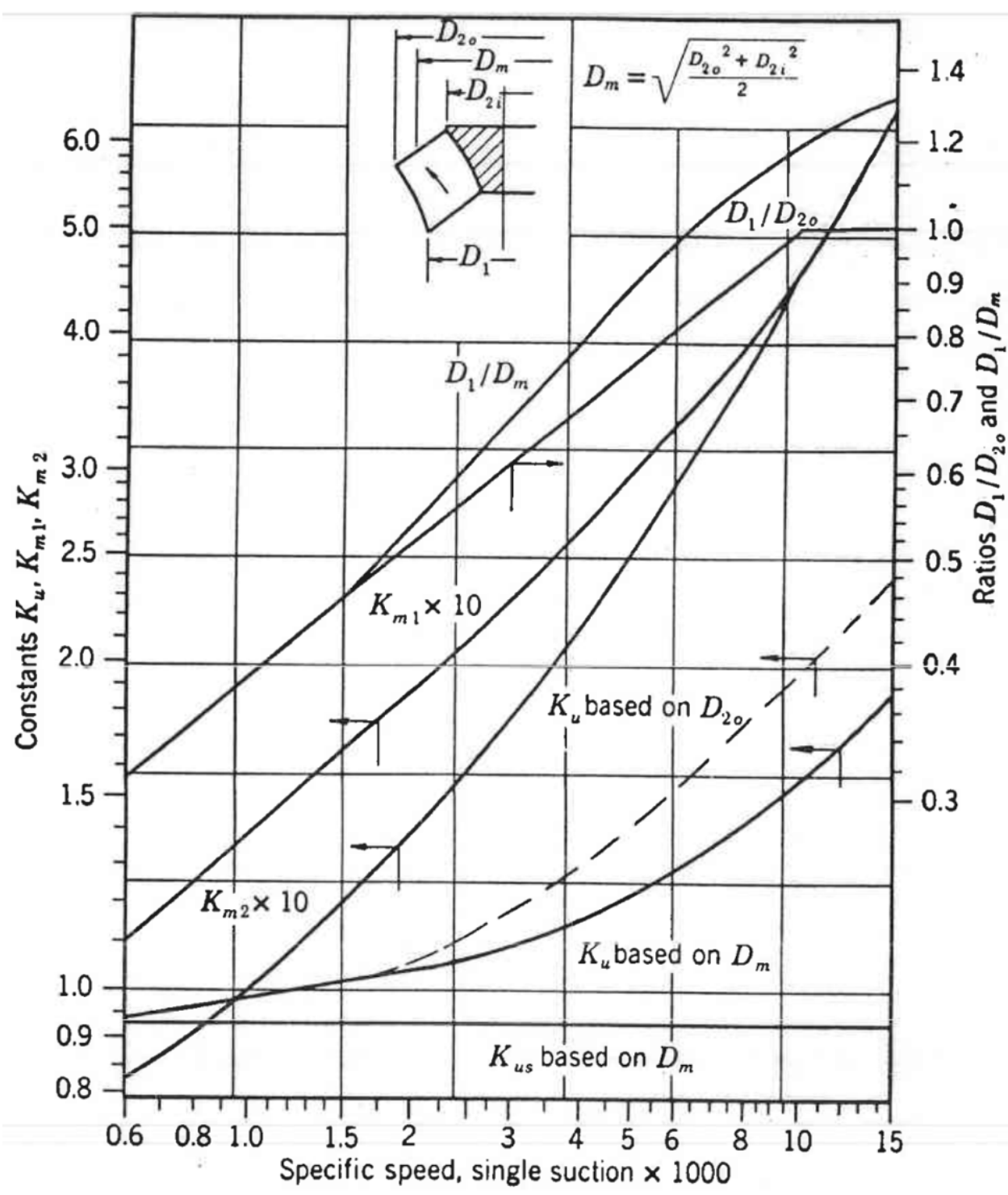

Figure 2-4. Impeller design constants $K_{m 1}$ and $K_{m 2}$ versus pump specific speed $\eta_{s}$. Reproduced from [24] with permission.

blade at discharge, $b_{2}$, can be determined (Appendix A, Equations A.1 - A.6).

The entrance blade tip speed, $u_{1}$, as well as the meridional, $C_{m 1}$, tangential, $C_{u 1}$, and total absolute, $C_{1}$, velocities of the fluid can be calculated by solving the entrance triangle, the absolute fluid entrance angle, $\alpha_{1}$, so the entrance blade angle, $\beta_{1}$, can be determined (Appendix A, Equations A.7 - A.12). To complete the solution of the entrance side of the impeller, the eye diameter $D_{1}$, and 
entrance blade height, $b_{1}$, were determined (Equations 2.6, 2.7). Tuzson [28] provides an equation to calculate the eye diameter for single-stage pumps with low specific speed $(<5000)$. All calculated entrance and discharge triangle parameter values for the Inspired Pediatric VAD impeller are shown in Table 2-2.

$$
\begin{gathered}
D_{1}=1.533\left(\frac{Q}{n}\right)^{\frac{1}{3}}=1.533\left(\frac{5.833 \times 10^{-5} \mathrm{~m}^{3} / \mathrm{sec}}{445.58 \mathrm{rad} / \mathrm{sec}}\right)^{\frac{1}{3}}=7.78 \mathrm{~mm} \rightarrow \mathbf{8 . 0 0 ~} \mathbf{~ m m} \\
b_{1}=\frac{Q}{\pi D_{1} C_{m 1}}=\frac{5.833 \times 10^{-5} \mathrm{~m}^{3} / \mathrm{sec}}{\pi \times 0.008 \mathrm{~m} \times 1.20 \mathrm{~m} / \mathrm{sec}}=1.93 \mathrm{~mm} \rightarrow 2.00 \mathrm{~mm}
\end{gathered}
$$

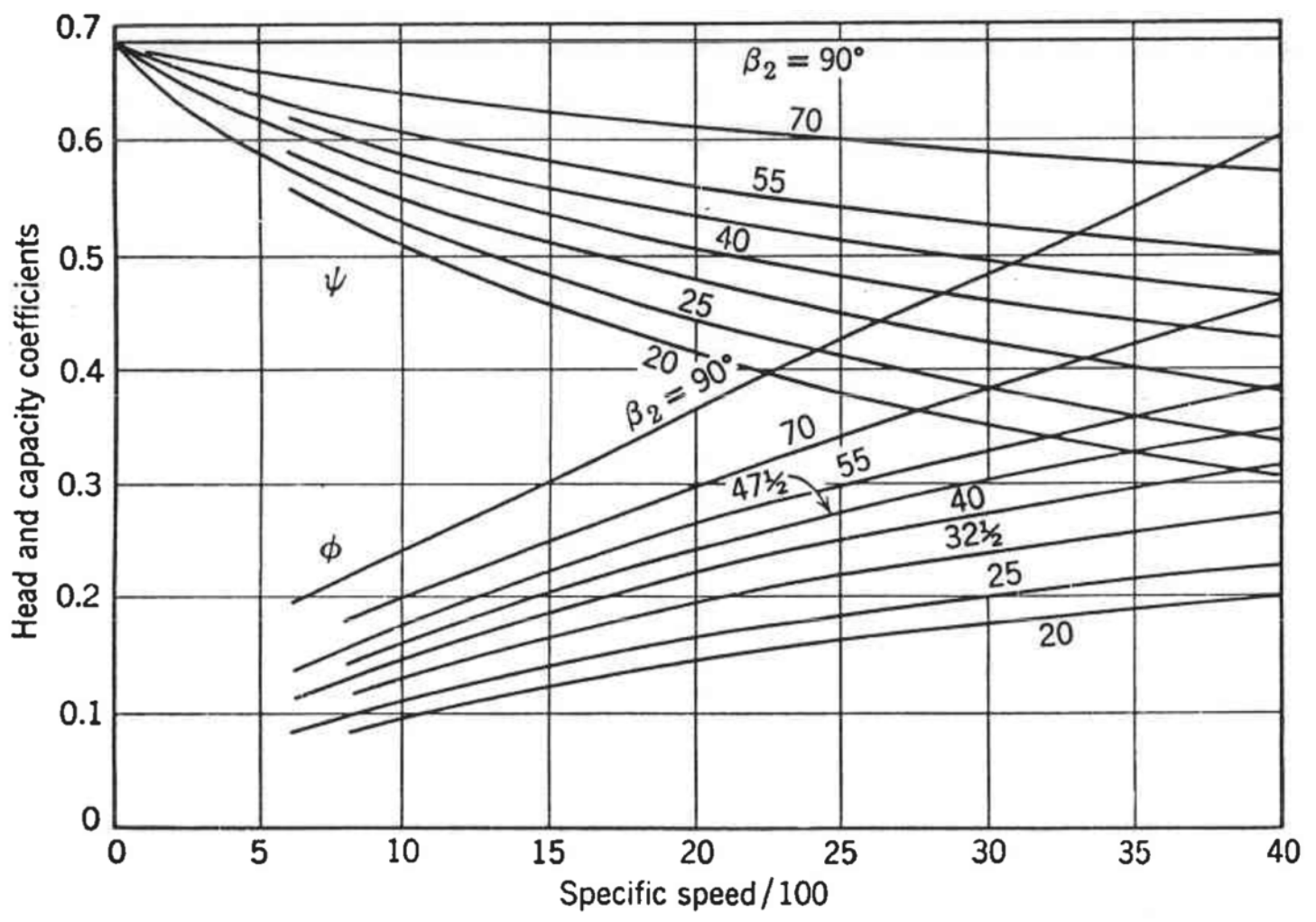

Figure 2-5. Head coefficient $\psi$ and flow coefficient $\phi$ versus pump specific speed $\eta_{s}$ for various blade exit angles $\beta_{2}$. Reproduced from [24] with permission. 
Table 2-2. All calculated parameter values for the entrance and discharge Euler velocity triangles under ideal flow conditions for the Inspired Pediatric VAD impeller.

\begin{tabular}{|c|c|c|c|c|c|}
\hline \multicolumn{3}{|c|}{ Blade Entrance Triangle } & \multicolumn{3}{|c|}{ Blade Discharge Triangle } \\
\hline Blade Tip Speed & $\begin{array}{c}u_{1} \\
(\mathrm{~m} / \mathrm{sec})\end{array}$ & 1.78 & Blade Tip Speed & $\begin{array}{c}u_{2} \\
(\mathrm{~m} / \mathrm{sec})\end{array}$ & 6.10 \\
\hline Meridional Velocity & $\begin{array}{c}C_{m 1} \\
(\mathrm{~m} / \mathrm{sec})\end{array}$ & 1.20 & Meridional Velocity & $\begin{array}{c}C_{m 2} \\
(\mathrm{~m} / \mathrm{sec})\end{array}$ & 0.85 \\
\hline Tangential Velocity & $\begin{array}{c}C_{u 1} \\
(\mathrm{~m} / \mathrm{sec})\end{array}$ & 0.002 & Tangential Velocity & $\begin{array}{c}C_{u 2} \\
(\mathrm{~m} / \mathrm{sec})\end{array}$ & 4.05 \\
\hline Total Absolute Velocity & $\begin{array}{c}C_{1} \\
(\mathrm{~m} / \mathrm{sec})\end{array}$ & 1.20 & Total Absolute Velocity & $\begin{array}{c}C_{2} \\
(\mathrm{~m} / \mathrm{sec})\end{array}$ & 4.14 \\
\hline Fluid Entrance Angle & $\begin{array}{l}\alpha_{1} \\
\left(^{\circ}\right)\end{array}$ & 89.90 & Fluid Discharge Angle & $\begin{array}{l}\alpha_{2} \\
\left(^{\circ}\right)\end{array}$ & 11.88 \\
\hline Blade Entrance Height & $\begin{array}{c}b_{1} \\
(m m)\end{array}$ & 2.00 & Blade Discharge Height & $\begin{array}{c}b_{2} \\
(\mathrm{~mm})\end{array}$ & 1.00 \\
\hline Blade Entrance Angle & $\begin{array}{l}\beta_{1} \\
\left(^{\circ}\right)\end{array}$ & 33.98 & Blade Discharge Angle & $\begin{array}{l}\beta_{2} \\
\left(^{\circ}\right)\end{array}$ & 22.50 \\
\hline
\end{tabular}

\subsection{Account for Flow Slip, Recirculation, and Pre-Rotation}

Under ideal conditions, flow passing through the impeller blade and exiting the blade tip will follow the flow path directed by the blade exit angle, $\beta_{2}$. In practice, with the addition of friction forces and other flow variances and losses, the exiting flow will follow a path that is less than ideal and "slip" ( $\alpha_{2}^{\prime}$ and $\left.\beta_{2}{ }^{\prime}\right)$ [28]. This is due, in part, to the relative circulation of flow particles between impeller blades, which reduces the tangential velocity, $C_{u}$, at the blade exit but increases 
at the blade entrance. To account for this, slip coefficient $S$ (Equations 2.8, 2.9) were applied to modify the Euler discharge velocity triangle (Figure 2-3E).

$$
\begin{gathered}
\frac{c_{u 2}{ }^{\prime}}{u_{2}}=\psi=S-\phi \tan \beta_{2} \\
S=\psi+\phi \tan \beta_{2}=0.515+0.115 \tan 22.50^{\circ}=0.563
\end{gathered}
$$

Applying the slip coefficient, predicted "actual" fluid velocity and angle values at the impeller discharge were calculated, including the actual tangential velocity, $C_{u 2}{ }^{\prime}$, total absolute velocity, $C_{2}{ }^{\prime}$, absolute fluid, $\alpha_{2}{ }^{\prime}$ and blade angles, $\beta_{2}{ }^{\prime}$ (Appendix A, Equations A.13 - A.16). Calculated parameter values for the modified discharge triangle are shown in Table 2-3. As expected, $\beta_{2}^{\prime}<\beta_{2}$, because the actual flow will always slip and lag behind the discharge angle of the blades due to the decrease in tangential velocity, $C_{u}$. For our Pediatric VAD, the blade discharge angle should remain $\beta_{2}=22.5^{\circ}$. The actual fluid discharge angle, $\alpha_{2}^{\prime}$, will become useful in determining the velocity within the pump volute.

To account for pre-rotation of the fluid at the blade entrance, a pre-rotation ratio, $R_{1}$, which is the ratio of the blade pitch-per-second, $P_{1 s}$, to the flow meridional velocity, $C_{m 1}$, was applied. Under ideal conditions, the velocity of the fluid at the impeller eye should equal $C_{m 1}$, and it is assumed that the flow will approach the impeller entrance with essentially 0 tangential velocity $\left(C_{u 1}\right.$ was calculated as $0.002 \mathrm{~m} / \mathrm{sec}$ ). However, the rotation of the impeller will impart some 
rotation to the surrounding fluid, causing the fluid to "pre-rotate" before entering the impeller eye, which increases the overall absolute fluid velocity (Figure 2-6).

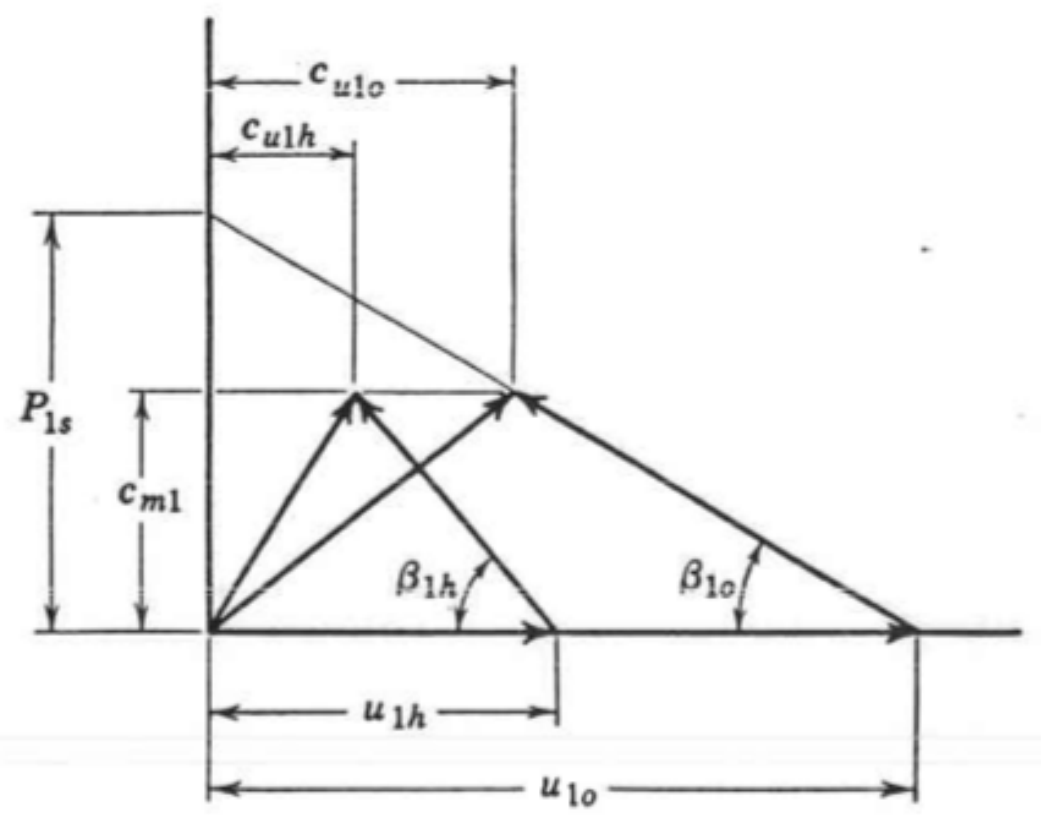

Figure 2-6. Impeller design constants $K_{m 1}$ and $K_{m 2}$ versus pump specific speed $\eta_{s .}$ Reproduced from [24] with permission.

The blade pitch-per-second is the required change in the blade angle of attack (or pitch) corresponding to $\beta_{1}$. Accounting for pre-rotation, $\beta_{1}$ should be increased from the initially calculated Euler value to better match the actual flow at the impeller eye. For single inlet suction pumps, Stepanoff [24] suggests the use of a ratio between 1.15 and 1.25. For the Inspired Pediatric VAD, $R_{1}=1.20$ was chosen to solve the modified inlet velocity triangle (Figure 2-3D). Applying the pre-rotation ratio, predicted "actual" fluid velocity and angle values at the impeller entrance can be calculated, including the actual tangential velocity, $C_{u 1}{ }^{\prime}$, 
total absolute velocity, $C_{1}{ }^{\prime}$. and blade entrance angle, $\beta_{1}{ }^{\prime}$ (Appendix $\mathbf{A}$, Equations A.17 - A.20) (Table 2-3).

Table 2-3. All calculated parameter values for the modified entrance and discharge Euler velocity triangles accounting for flow pre-rotation and slip for the Inspired Pediatric VAD impeller.

\begin{tabular}{|c|c|c|c|c|c|}
\hline \multicolumn{3}{|c|}{ Modified Blade Entrance Triangle } & \multicolumn{3}{|c|}{ Modified Blade Discharge Triangle } \\
\hline Blade Pitch Per Second & $\begin{array}{c}P_{1 \mathrm{~s}} \\
(\mathrm{~m} / \mathrm{sec})\end{array}$ & 1.44 & Actual Tangential Velocity & $\begin{array}{c}\mathrm{Cu2}^{\prime} \\
(\mathrm{m} / \mathrm{sec})\end{array}$ & 3.14 \\
\hline Actual Tangential Velocity & $\begin{array}{c}C_{u 1^{\prime}} \\
(\mathrm{m} / \mathrm{sec})\end{array}$ & 0.35 & $\begin{array}{r}\text { Actual Total Absolute } \\
\text { Velocity }\end{array}$ & $\begin{array}{c}\mathrm{C}_{2}^{\prime} \\
(\mathrm{m} / \mathrm{sec})\end{array}$ & 3.25 \\
\hline $\begin{array}{r}\text { Actual Total Absolute } \\
\text { Velocity }\end{array}$ & $\begin{array}{c}C_{1}^{\prime} \\
(m / s e c)\end{array}$ & 1.25 & $\begin{array}{r}\text { Actual Blade Discharge } \\
\text { Angle }\end{array}$ & $\begin{array}{l}\beta_{2}^{\prime} \\
\left(^{\circ}\right)\end{array}$ & 16.02 \\
\hline $\begin{array}{r}\text { Actual Blade Entrance } \\
\text { Angle }\end{array}$ & $\begin{array}{l}\beta_{1}^{\prime} \\
\left({ }^{\circ}\right)\end{array}$ & 40.00 & $\begin{array}{r}\text { Actual Fluid Discharge } \\
\text { Angle }\end{array}$ & $\begin{array}{l}\alpha_{2}^{\prime} \\
\left({ }^{\circ}\right)\end{array}$ & 15.15 \\
\hline
\end{tabular}

\subsection{Plot Impeller Blade Shape}

Pfleiderer [25] demonstrated that the optimum number of impeller blades, $Z$, for a given design can be estimated using Equations 2.10 and 2.11, which for the the Inspired Pediatric VAD resulted in 6 blades.

$$
\begin{gathered}
\beta_{m}=\frac{\beta_{1}+\beta_{2}}{2}=\frac{40.00^{\circ}+22.5^{\circ}}{2}=31.25^{\circ} \\
Z=6.5 \frac{D_{2}+D_{1}}{D_{2}-D_{1}} \sin \beta_{m}=6.5 \frac{27.4+8}{27.4-8} \sin 31.25^{\circ}=6.15 \rightarrow \mathbf{6} \text { Blades }
\end{gathered}
$$


The number of impeller blades directly impacts the level of flow recirculation and subsequent losses between the blades (as well as flow slip). Increasing the number of impeller blades will decrease the amount of flow recirculation, but too many impeller blades will lead to other efficiency losses due to flow restriction [31]. Choosing the correct number of blades is a critical parameter for the design. Qian et al. [32] showed that when backward swept centrifugal impeller designs with between 5 and 7 blades were analyzed, an impeller containing 6 blades achieved the lowest level of hemolysis.

Kyparissis et al. [33] demonstrated how to plot the curve of an impeller blade in two-dimensions using a simple-arc method (SAM). One arc acting as the blade mean line is drawn between the pump inlet and outlet diameters between points $A$ and $C$ (Figure 2-7A). An auxiliary circle $C_{a}$ with diameter $d_{1}$ is drawn in the center of the impeller plot (Equation 2.12). Line $E$ is then drawn intersecting point $A$ and tangent to circle $C_{a}$. Line $E$ has length $R$, which is the radius of the mean blade arc (Equation 2.13).

$$
\begin{gathered}
d_{1}=D_{1} \sin \beta_{1}=5.14 \mathrm{~mm} \\
R=\frac{1}{2} \times \frac{\left(\frac{D_{2}}{2}\right)^{2}-\left(\frac{D_{1}}{2}\right)^{2}}{\left(\frac{D_{2}}{2}\right) \cos \beta_{2}-\left(\frac{D_{1}}{2}\right) \cos \beta_{1}}=\frac{1}{2} \times \frac{\left(\frac{27.4 \mathrm{~mm}}{2}\right)^{2}-\left(\frac{8 m m}{2}\right)^{2}}{\left(\frac{27.4 m m}{2}\right) \cos 22.5^{\circ}-\left(\frac{8 m m}{2}\right) \cos 40^{\circ}}=8.956 \mathrm{~mm}
\end{gathered}
$$

For our Pediatric VAD, SAM was plotted (Figure 2-7B and C) and a threedimensional model of these values was constructed (Figure 2-7D). 
With the preliminary blade dimensions and shape set, a method to evaluate the design, as well as the blade entrance and discharge heights ( $b_{1}$ and $b_{2}$ ), was used to calculate the cross-sectional flow area at the impeller entrance $\left(\mathrm{a}_{1}\right)$ and discharge $\left(\mathrm{a}_{2}\right)$ (Figure 3D) using Equations 2.14 and 2.15.

$$
\begin{aligned}
& a_{1}=\frac{2 \pi \frac{D_{1}}{2} b_{1}}{Z}=\frac{2 \pi \frac{27.4 m m}{2} 1 \mathrm{~mm}}{6}=14.347 \mathrm{~mm}^{2} \\
& a_{2}=\frac{2 \pi \frac{D_{2}}{2} b_{2}}{Z}=\frac{2 \pi \frac{8 m m}{2} 2 \mathrm{~mm}}{6}=8.378 \mathrm{~mm}^{2}
\end{aligned}
$$

The ratio of entrance and discharge flow areas $\left(a_{2} / a_{1}\right)$ is directly related to the impeller hydraulic efficiency. A smooth transition between areas helps maintain a high hydraulic efficiency and traditionally an area ratio close to unity, between 1 and 1.3, is optimum [34]. For the Inspired Pediatric VAD impeller design, $a_{2} / a_{1}=1.7$. By increasing the entrance blade height $b_{1}$ from $2 \mathrm{~mm}$ to $3 \mathrm{~mm}$, the entrance and discharge flow areas will be almost equal $\left(a_{2} / a_{1}=1\right)$. However, Curtas et al. [35] demonstrated that for miniaturized pumps with low specific speed, larger discharge areas $\left(a_{2} / a_{1}>1\right)$ lead to reduced meridional fluid velocity $C_{m 2}$ at the exit, and in turn, generated a higher acheivable pump head. For the initial Inspired Pediatric VAD impeller design, the inlet and outlet blade heights will remain as calculated $\left(b_{1}=2 \mathrm{~mm}, b_{2}=1 \mathrm{~mm}\right)$, but modification of these heights during future evaluation will be critical to achieving higher pressure head. 


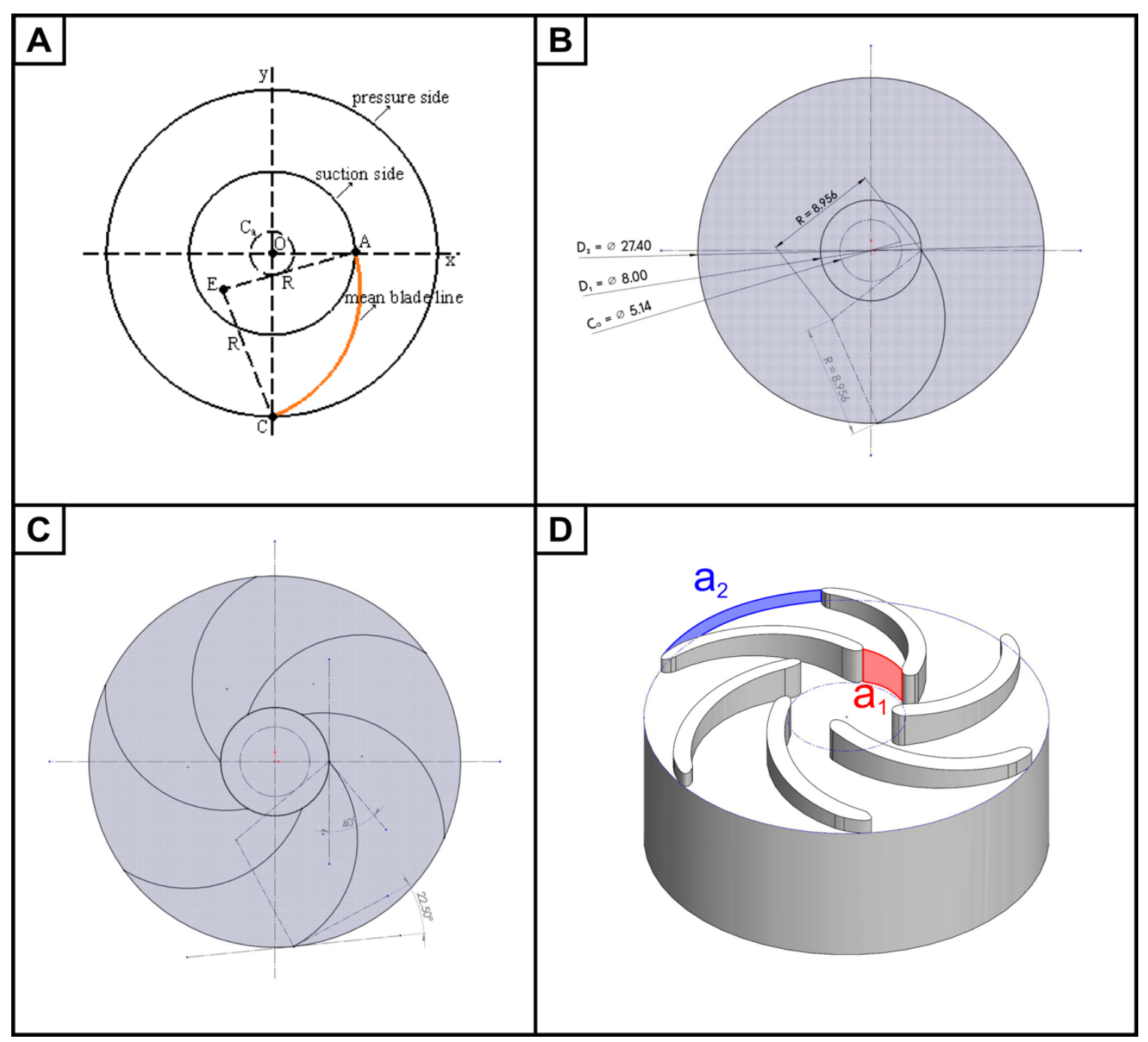

Figure 2-7. (A) Representation of the mean blade line plotted using the simplearc method (SAM), reproduced from [33] with permission, (B) Plot of the mean blade line for the Inspired Pediatric VAD impeller, (C) Plot of all six mean blade lines for the Inspired Pediatric VAD impeller with dimensioned entrance and exit angles, (D) Three-dimensional solid model of the basic Inspired Pediatric VAD impeller features.

\subsection{Impeller Blade Thickness and Profiling}

Acheivable blade thickness $(e)$ is typically determined by the manufacturing process of the impeller and mechanical requirements (forces) of the blades. The Inspired Pediatric VAD impeller will be produced using injection 
molding of a biocompatable polycarbonate polymer and a blade thickness of around $1 \mathrm{~mm}$ will be the smallest achievable dimension. According to traditional theory, blade thickness should be as small as possible to ensure that the desired flow lines are achieved [36]; all calculations of the flow leading up this point in our design have assumed a blade with zero thickness (i.e. a thin line). Shigemitsu et al. [37] demonstrated that acheivable pump head and efficiency increased with the decrease in blade thickness for a miniature centrifugal pump with six backward swept blades. Conversly, Kim et al. [38] showed that an impeller with unusually thick blades provided increased efficiency and reduced hemolysis, though this was determined to be more of a factor of the blade tip clearance than the blade thickness itself. For the preliminary design, the Inspired Pediatric VAD will have blades with a continuous thickness of $1 \mathrm{~mm}$.

Profiling the leading and trailing edges of the blades is also critical to maintaining the desired flow lines and angles. For Inspired impeller, the leading edge was profiled with a uniform elipse along the mean line of the blade (Figure 2-8A) to ensure favorable flow and pressure distributions [31]. The trailing edge was profiled with a non-uniform elipse and was filed on the suction side of the blade, known as under-filing (Figure 2-8A). This helps to decelerate the flow on the suction side of the blade tip which subsequently increases the achievable pump head [31], [39]. All calculated critical parameters for the initial Inspired Pediatric VAD impeller design are summarized in Table 2-4. 


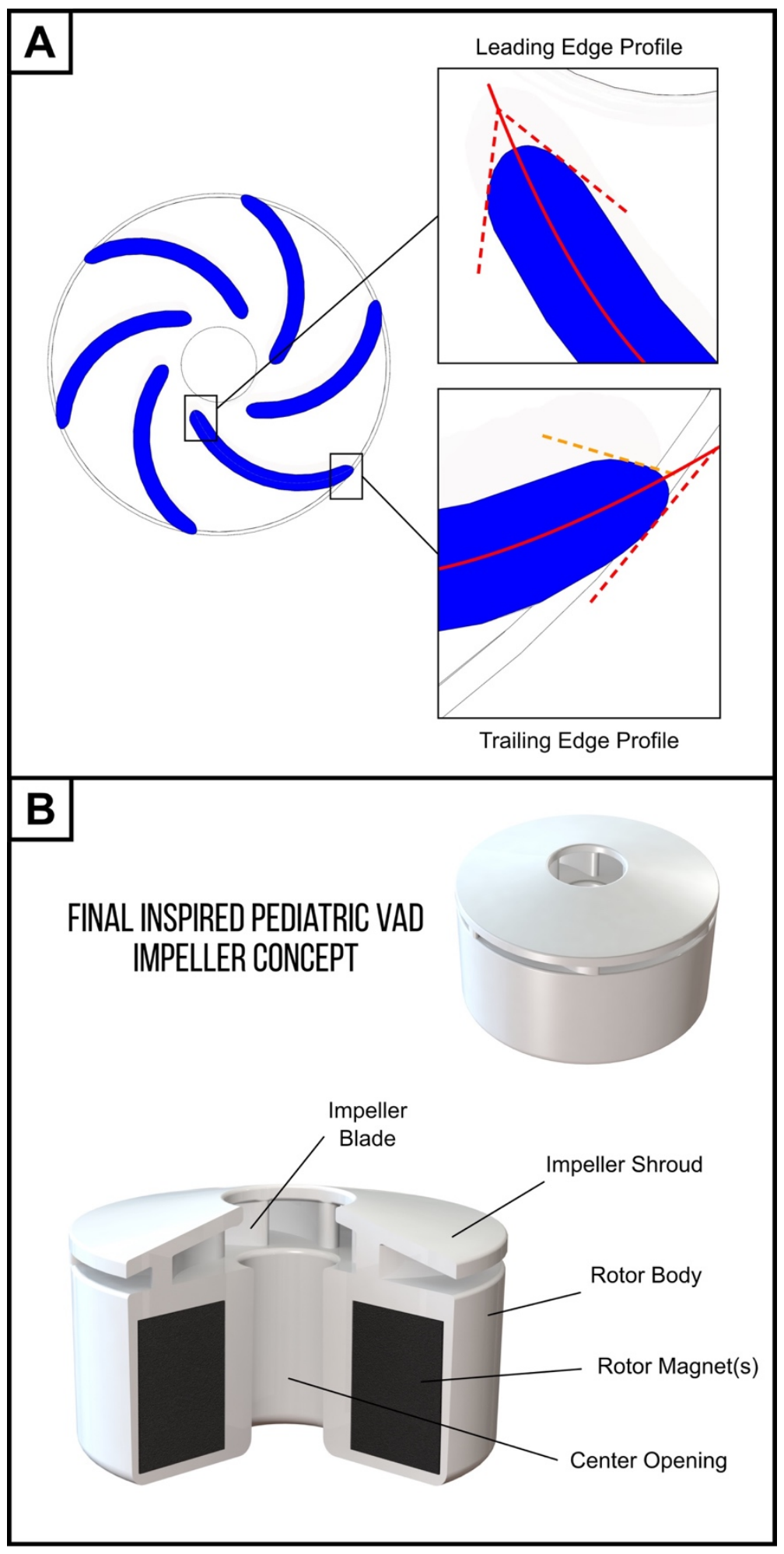

Figure 2-8. (A) Detailed view of impeller blade shape, thickness, and tip profiling, (B) Rendering of the final Inspired Pediatric VAD impeller concept with details including the impeller shroud, impeller blades, rotor body, rotor magnetics, and rotor body center opening. 
Table 2-4. All critical calculated impeller dimensions for the Inspired Pediatric VAD.

\begin{tabular}{|c|c|c|c|}
\hline \multicolumn{4}{|c|}{ Inspired Pediatric VAD Calculated Impeller Dimensions } \\
\hline Parameter & Symbol & Units & Value \\
\hline Impeller Inner/Eye diameter & $\mathrm{D}_{1}$ & $\mathrm{~mm}$ & 8.00 \\
\hline Impeller Outer Diameter & $\mathrm{D}_{2}$ & $\mathrm{~mm}$ & 27.40 \\
\hline $\begin{array}{c}\text { Estimated Rotational Speed at } \\
\text { Design Point }\end{array}$ & $\mathrm{N}$ & RPM & 4255.00 \\
\hline Blade Inlet Angle & $\beta_{1}^{\prime}$ & $\circ$ & 40.00 \\
\hline Blade Outlet Angle & $\beta_{2}$ & $\circ$ & 22.50 \\
\hline Blade Inlet Height & $b_{1}$ & $\mathrm{~mm}$ & 2.00 \\
\hline Blade Outlet Height & $b_{2}$ & $\mathrm{~mm}$ & 1.00 \\
\hline Blade Thickness & e & $\mathrm{mm}$ & 1.00 \\
\hline Estimated Blade Number & Z & blades & 6.00 \\
\hline Blade Mean Curve Radius & $\mathrm{R}$ & $\mathrm{mm}$ & 8.92 \\
\hline Impeller Head Coefficient & $\psi$ & & 0.52 \\
\hline Impeller Flow Coefficient & $\varphi$ & & 0.12 \\
\hline Speed Number & $\sigma$ & & 0.12 \\
\hline Diameter Number & $\delta$ & & 7.89 \\
\hline Specific Speed & $\eta_{\mathrm{s}}$ & & 1019.50 \\
\hline
\end{tabular}

\subsection{Additional Impeller Features}

To finish the preliminary impeller design, additional features were added including a rotor body below the impeller blades, a shroud on top of the impeller blades, and a center flow opening in the middle of the rotor body (Figure 2-8B). 
The rotor body was added to house the magnetic components needed for the MagLev motor system, approximately $12 \mathrm{~mm}$ in height below the impeller.

A blade shroud was added, resulting in a "closed" impeller design, to help reduce the amount of flow recirculation and leakage from blade to blade and increase overall hydraulic efficiency [40]. However the shroud can induce retrograde flow between the shroud top surface and the top surface of the pump housing [41], which can be a benefit by increasing surface washing of these areas to reduce risk of thrombus formation but it can also lead to decreased efficiency and increased flow pre-rotation at the impeller eye. Special attention will need to be considered at this area when designing the pump housing and volute to ensure the optimal axial gap between the impeller shroud and upper housing.

The center opening in the rotor body was added to act as a "balancing hole" to reduce the amount of axial thrust, or lift, produced by the impeller when rotating [36]. Adding the central opening simplifies the MagLev motor design because the motor will have less of a need to counter the axial force of the impeller to keep it centered in the flow channel, resulting in less power consumption. Additionally, the central opening will increase the flow around the rotor body surface, eliminating flow stagnancy between the rotor body and pump housing with increased surface washing and the potential to reduce thrombus formation [42]. However, this secondary flow channel, similar to that caused by the impeller shroud, may lead to increased flow recirculation within the pump and a decrease in overall hydraulic efficiency [28]. Special consideration in designing 
the radial gap between the rotor body and pump housing will be applied to ensure that the benefits of this secondary flow don't also induce extreme efficiency reduction.

\subsection{Conclusion}

The goal of this manuscript is to present step-by-step procedures used in designing the centrifugal pump impeller for the Inspired Pediatric VAD by applying well-established traditional pump theory. The procedures and example calculations were used to create an initial impeller design for the Inspired Pediatric VAD but may also be applied to the general design of other centrifugal pump impellers. Essential considerations for how to design the critical dimensions for the impeller blades were presented, for which a functional impeller for the Inspired Pediatric VAD was successfully developed. The development methods described in this article are only the beginning in a long and complex process in designing radial blood pumps. The next development steps include the calculation and design of the pump volute, pump casing including all radial and axial gaps and clearances, and pump inlet and outlet conduits. After all initial geometries are determined, the hydraulic performance and hemocompatibility of the pump design will be rigorously evaluated using CFD predictions and prototype experiments. The data and lessons learned from these next stages will inform iterative design changes and improvements.

We intend to report in future communications the next steps to fully characterize the Pediatric VAD performance experimentally using in vitro (mock 
flow loop) and in vivo (ovine) models. In applying this approach, we were able to design a novel pediatric pump impeller as the next step in the development process toward fulfilling our long-term goal of achieving design freeze of a clinical grade device to treat a pediatric population in desperate need of temporary (30day) extracorporeal MCS therapy. A parallel effort has been undertaken to develop the universal controller that will drive the pediatric pump. The next step is to combine the two components to demonstrate and assess the performance of the system when the controller is used to drive the pediatric device. 


\section{CHAPTER 3 : DESIGN AND COMPUTATIONAL EVALUATION OF A PEDIATRIC MAGLEV ROTARY BLOOD PUMP}

\subsection{Introduction}

In this article, we discuss the initial conceptual design and computational predictions for a new mechanical circulatory support option; the Inspired Universal MagLev System and the Inspired Pediatric VAD. The vision for this system is a universal controller/motor which can be paired with many different single-use pump heads for up to 30-days of circulatory or potential respiratory support. The development goals for the system are to make a product that is smaller and lower-cost than current offerings clinically in use and is selfcontained to allow for patient ambulation during treatment.

Pediatric heart failure (HF) patients have historically been an underserved population with few technological advancements successfully translated into

Authors: ${ }^{1}$ Landon H. Tompkins, MEng., ${ }^{2}$ Barry N. Gellman M.S., ${ }^{3}$ Gino F. Morello, ${ }^{4}$ Steven R. Prina Ph.D., ${ }^{1}$ Thomas Roussel Ph.D., ' Jonathan A. Kopechek Ph.D., ${ }^{2}$ Priscilla C. Petit,

${ }^{5}$ Mark S. Slaughter M.D., ${ }^{1,5}$ Steven C. Koenig Ph.D., ${ }^{1,2,5}$ Kurt A. Dasse Ph.D.

Department of Bioengineering, University of Louisville, Louisville, $K Y$

Inspired Therapeutics LLC, Merritt Island, FL

Veritium Research LLC, Fort Lee, NJ

BLDC Designs LLC, Rocklin, CA

Department of Cardiovascular and Thoracic Surgery, University of Louisville, Louisville, $K Y$ 
clinical practice. The combination of $17 \%$ mortality for pediatric HF patients listed for heart transplantation [11] and limited alternative treatment options, has led to the emergence of mechanical circulatory support (MCS) an important modality for improving survival and maintaining growth and development in pediatric patients awaiting transplantation [9]. Unfortunately, development of suitable MCS devices for pediatric patients has been severely limited due to a variety of engineering and socioeconomic barriers. Creating a one-size-fits-all device is inadequate due to the wide variation in patient size and age, multiple congenital malformations and etiologies of cardiomyopathy, and the overall lack of data identifying the needs of the pediatric HF population [43].

Current ventricular assist devices (VADs) feature radial or axial impeller designs to provide high-output continuous blood flow (CF) for the adult HF patient population [44]. Despite increasing clinical demand, the development and clinical translation of pediatric-specific CF VADs has been limited. Surgeons often resort to using commercially available adult VADs, such as the HeartWare HVAD or Abbott Heartmate 3, to fill this void, but the mismatch of adult device requirements versus pediatric patient needs has raised the potential for clinically significant adverse events (thrombus formation and neurological events) at unacceptable rates [45]. Extracorporeal VADs represent an excellent option for pediatric patients since the size and clinical needs of these patients can vary widely. External devices allow flexibility to implement a wide range of circulatory support without the need for invasive surgery. Current extracorporeal VADs used for pediatric support include the Berlin Heart EXCOR, Medtronic Bio-Pump BP- 
50, Maquet RotaFlow, Medos Deltastream DP3, and the Thoratec

CentriMag/PediMag systems [46]. A comparison of the design features of these

pumps including their limitations is summarized in Table 3-1.

Table 3-1. Summary of design features of the Inspired Pediatric VAD compared to current commercially available pediatric extracorporeal VADs: Berlin Heart EXCOR, Medtronic Bio-Pump BP-50, Maquet RotaFlow, Medos Deltastream DP3 and Thoratec PediMag.

\begin{tabular}{|c|c|c|c|c|c|}
\hline \multicolumn{6}{|c|}{ Comparison of Pediatric Extracorporeal Mechanical Circulatory Assist Devices } \\
\hline Device & $\begin{array}{l}\text { Rotor/lmpeller } \\
\text { Diameter } \\
(\mathrm{mm})\end{array}$ & $\begin{array}{c}\text { Priming } \\
\text { Volume } \\
(\mathrm{mL})\end{array}$ & Bearing & Motor Drive & Limitations \\
\hline $\begin{array}{l}\text { Berlin Heart } \\
\text { EXCOR } \\
{[47],[48]}\end{array}$ & $\begin{array}{c}\text { NA } \\
\text { Sac Type } \\
\text { Pump }\end{array}$ & $10-60$ & $\begin{array}{c}\text { NA } \\
\text { Pneumatically } \\
\text { Driven }\end{array}$ & $\begin{array}{l}\text { Polyurethane } \\
\text { flexible } \\
\text { membrane }\end{array}$ & $\begin{array}{l}\text { Pneumatically driven - } \\
\text { pulsatile device. Limited } \\
\text { long-term use and } \\
\text { durability. High risk of } \\
\text { stroke. Requires large } \\
\text { bedside motor/controller. }\end{array}$ \\
\hline $\begin{array}{l}\text { Medtronic } \\
\text { Bio-Pump } \\
\text { BP-50 [49] }\end{array}$ & 79 & 48 & $\begin{array}{l}\text { Polycarbonate } \\
\text { Journal } \\
\text { Bearing }\end{array}$ & $\begin{array}{l}\text { Magnetic } \\
\text { Coupling }\end{array}$ & $\begin{array}{l}\text { Shafts, seals and bearings } \\
\text { lead to lower durability. } \\
\text { Only capable of up to } 1.5 \\
\text { L/min. Requires large } \\
\text { bedside motor/controller. }\end{array}$ \\
\hline $\begin{array}{l}\text { Maquet } \\
\text { RotaFlow } \\
\text { [18], [50] }\end{array}$ & 49 & 35 & $\begin{array}{c}\text { Stainless } \\
\text { Steel/Sapphire } \\
\text { Pivot Bearing }\end{array}$ & $\begin{array}{l}\text { Magnetic } \\
\text { Coupling }\end{array}$ & $\begin{array}{l}\text { Decreased efficiency } \\
\text { under low flow conditions } \\
\text { (Pediatric and ECMO } \\
\text { range). Requires large } \\
\text { bedside motor/controller. }\end{array}$ \\
\hline $\begin{array}{c}\text { Medos } \\
\text { Deltastream } \\
\text { DP3 [51],[52] }\end{array}$ & 25 & 16 & $\begin{array}{l}\text { Ceramic } \\
\text { Bearing }\end{array}$ & $\begin{array}{l}\text { Magnetic } \\
\text { Coupling }\end{array}$ & $\begin{array}{l}\text { Diagonal flow impeller. } \\
\text { Decreased efficiency at } \\
\text { low flow (Pediatric and } \\
\text { ECMO range). Requires } \\
\text { large bedside } \\
\text { motor/controller. }\end{array}$ \\
\hline $\begin{array}{c}\text { Thoratec } \\
\text { PediMag } \\
\text { [53] }\end{array}$ & 32.5 & 14 & Bearingless & $\begin{array}{c}\text { True } \\
\text { Magnetic } \\
\text { Levitation }\end{array}$ & $\begin{array}{l}\text { Only approved for low } \\
\text { flows }(1.5 \mathrm{~L} / \mathrm{min}) \text {. } \\
\text { Requires large bedside } \\
\text { motor/controller. }\end{array}$ \\
\hline $\begin{array}{l}\text { Inspired } \\
\text { Pediatric } \\
\text { VAD }\end{array}$ & 27.4 & 9.5 & Bearingless & $\begin{array}{c}\text { True } \\
\text { Magnetic } \\
\text { Levitation }\end{array}$ & $\begin{array}{l}\text { Limited to flow rates up to } \\
3.5 \mathrm{~L} / \mathrm{min}\end{array}$ \\
\hline
\end{tabular}


To meet the clinical needs of the pediatric patient population, Inspired Therapeutics (Merritt Island, FL) and the University of Louisville (Louisville, KY) have worked to identify and overcome potential limitations associated with extracorporeal VADs with the goal to incorporate significant engineering improvements to enhance the quality of life for these patients and their caretakers. We are developing a universal magnetically levitated (MagLev) extracorporeal pumping system, the Inspired Universal MagLev System (Figure 3-1), to address multiple indications for use (systemic circulatory support and potential respiratory support) for the entire HF patient population (neonate, pediatric and adult). The system is based on a single, universal controller paired with a MagLev motor. Single-use pump heads for different indications for use can be installed in the universal controller and motor to address a variety of needs (left and right heart support, ECMO, bypass, etc.) (Figure 3-2). This modular approach may offer a significant advancement over existing devices and will focus on the development of pumps (single-use pump heads) specific to each population and indication. The approach offers potential benefits including the reduction of human error by enabling healthcare providers to train on a single system, allow patient ambulation on an extracorporeal device, a decreased recovery time, and a reduction in the overall cost of care. Advantages of the Inspired System are summarized in Table 1-1.

This article focuses on the development of the first single-use pump head for this system, the Inspired Pediatric VAD. The pump head is specifically designed to address the needs of pediatric patients with diseases such as 


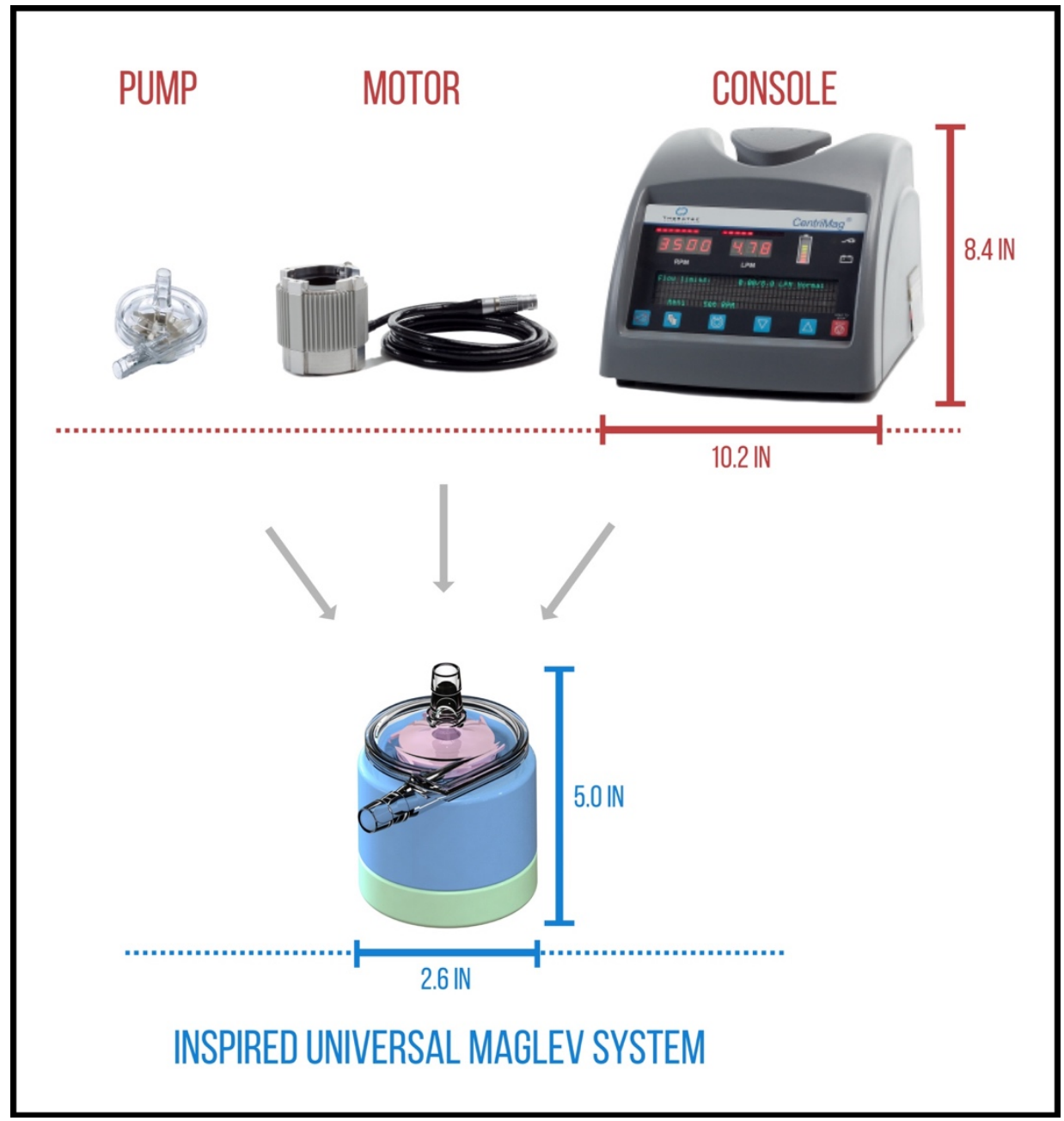

Figure 3-1. Components of the Thoratec CentriMag/PediMag system (pump, motor, controller) (Top) (with dimensions shown), compared to the Inspired Pediatric VAD and Universal MagLev System (Bottom) (with goal dimensions shown). The Inspired Universal System combines all extracorporeal components into one small, universal package allowing for potential greater ease of use, quality of care, and possible patient ambulation.

cardiomyopathy, myocarditis, and congenital malformations. The goals for the device are to provide high flow $(3.5 \mathrm{~L} / \mathrm{min})$ with a low priming volume $(9.5 \mathrm{~mL})$ and, when coupled with the Universal MagLev System, a small, compact form factor with the potential to be battery-powered and wearable. This article also outlines the criteria used to define the design input requirements for the Inspired 
Pediatric VAD (flows and pressures), details the methods and processes used to design two versions of the pump impeller and housing (Pump V1 and an improved version, Pump V2), and describes the methods used to confirm both designs using computational fluid dynamics (CFD) simulations.

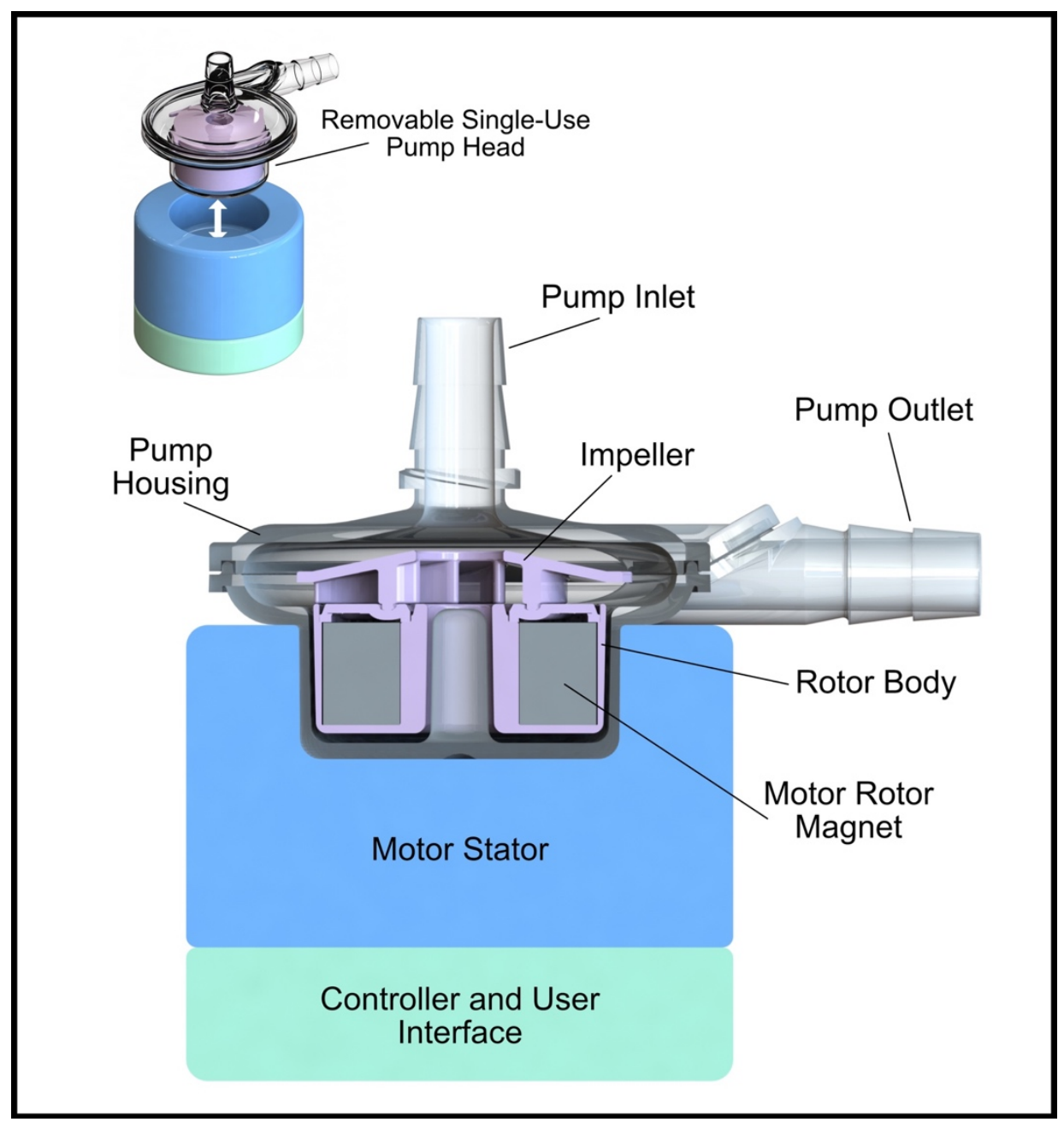

Figure 3-2. Basic components of the Inspired Universal MagLev system and removable Pediatric VAD pump head. 


\subsection{Methods}

\subsubsection{Design Criteria}

The Inspired Pediatric VAD is intended for use in neonate and pediatric patients weighing up to $40 \mathrm{~kg}$. Commercially available adult pumps have been shown to function adequately for older pediatric patients with weights exceeding $40 \mathrm{~kg}$ which served as the rationale for our focus on patients below this threshold. Healthy pediatric blood pressures can range from (1) $85 / 37 \mathrm{mmHg}$ (systolic/diastolic) for average weight of $10 \mathrm{~kg}$ at 1-year to (2) 106/62 $\mathrm{mmHg}$ for average weight of $40 \mathrm{~kg}$ at 12 years [54]-[57]. Patients with hypertension can experience systolic pressures between 110 and $135 \mathrm{mmHg}$ in this weight range [54]. Cardiac outputs for healthy pediatric patients range from 1.5 to $4.0 \mathrm{~L} / \mathrm{min}$ [57], [58]. Based upon these criteria, the Pediatric VAD is designed to provide 0.5-3.5 L/min of continuous flow at pressures up to $150 \mathrm{mmHg}$ to achieve adequate end organ perfusion (Table 3-3).

Table 3-2. Summary of proposed Inspired Pediatric VAD operational and design criteria.

\begin{tabular}{|cc|}
\hline Criteria & Value \\
\hline Pressure $(\mathrm{mmHg})$ & $\leq 150$ \\
Flow Rate (L/min) & $0.5-3.5$ \\
Rotational Speed (RPM) & $500-6000$ \\
Rotor Diameter (mm) & 27.4 \\
Inlet/Outlet Diameter (inch) & 0.25 \\
\hline
\end{tabular}


The diameter of the pump impeller/rotor is a critical parameter in achieving the desired flow (3.5 L/min) and performance at the pressure upper limit (150 $\mathrm{mmHg}$ ). A nominal impeller outside diameter of $27.4 \mathrm{~mm}$ was selected based on the desire for the pump to be significantly smaller, more compact, and hydraulically efficient compared to currently available pediatric extracorporeal, rotary pumps: BP-50, Rotaflow, and PediMag use larger diameter impellers of 79 $\mathrm{mm}, 49 \mathrm{~mm}$, and $32.5 \mathrm{~mm}$, respectively. Impeller diameter was selected to enable a rotor magnet diameter of 1 inch $(25.4 \mathrm{~mm})$ and a plastic casing surrounding the magnet with $1 \mathrm{~mm}$ thick walls, to provide a $15 \%$ reduction in diameter and a $30 \%$ reduction in priming volume compared to the PediMag pump (Table 3-1). Inlet and outlet port barb sizes of $1 / 4$ inch were chosen based on currently available pediatric cannula sizes [59], [60].

\subsubsection{Calculations for Impeller Design}

Dimensional analysis methods were used to determine impeller dimensions at the desired peak operating point, where maximum desired flow rate, $Q$, at pressure, $\Delta P$, is achieved. For the previously derived design criteria, this point is at a flow rate of $\mathrm{Q}=3.5 \mathrm{~L} / \mathrm{min}$ at a pressure of $\Delta \mathrm{P}=150 \mathrm{mmHg}$ with a $27.4 \mathrm{~mm}$ diameter impeller. The design methods of Cordier [23] were used to estimate the rotational speed of the impeller at 4255 RPM at the desired operating point.

The dimensions of the impeller blades were calculated using the design methods of Stepanoff [36] and by using Euler velocity triangles (triangles formed 
by the velocity vectors of the impeller blades and fluid) to determine the blade inlet and outlet angles $\left(\beta_{1}, \beta_{2}\right)$, fluid meridional and tangential velocities, and blade inlet $\left(b_{1}\right)$ and outlet heights $\left(b_{2}\right)$, as well as the eye diameter (inner diameter) of the impeller $\left(D_{1}\right)$. Hydraulic efficiency $\left(n_{H}\right)$ was calculated based on generated pressure $(\Delta \mathrm{P})$ and torque $(\tau)$ of the impeller:

$$
\eta_{H}=\frac{Q \times \Delta P}{\tau \times n}
$$

\subsubsection{Computational Fluid Dynamics Analysis}

Computational fluid dynamics (CFD) analysis techniques were used to evaluate and confirm the impeller and pump designs (V1, V2). Based on all calculated parameters, three-dimensional geometries (impeller and pump casing) were generated using commercial computer aided design (CAD) software (SolidWorks 2019, Dassault Systèmes, Waltham, MA). The inlet and outlet of the pump casing geometry were capped (virtually sealed off) to establish the computational fluid volume, which included pump inlet and outlet ports, pump volute, and all surfaces of the impeller. The pump geometry was transferred into SolidWorks Flow Simulation, a parametric flow simulation tool (FloEFD, Siemens, Munich, Germany) that applies the finite volume method [61] to solve the threedimensional Navier-Stokes equations [62]. Boundary conditions were set for the simulation as follows: flow velocity with parabolic profile at pump inlet, static pressure at pump outlet, and a real-wall stator condition (no-slip) for all pump casing walls. A rotating region was defined surrounding the pump impeller and 
the sliding-mesh method was used [63]. Blood was defined as a Newtonian fluid with a density of $\rho=1003 \mathrm{~kg} / \mathrm{m}^{3}$ at $37^{\circ} \mathrm{C}$ and a viscosity of $\mu=0.0035 \mathrm{~Pa}$-s. Although blood is considered a non-Newtonian fluid, it is shear thinning, and at the high shear rates (>100/s) typically found in rotary blood pumps, it behaves as an incompressible Newtonian fluid [64]. Therefore it is appropriate to model blood as a Newtonian fluid with acceptance of this assumption supported by other related CFD studies [65]-[67].

Reynolds numbers $(\mathrm{Re})$ were calculated [68], [69] for the pump inlet and impellers (V1, V2) to determine if turbulent flow could be expected (Appendix B, Table B-1). All calculated Reynolds numbers for the impellers were in the laminar flow range. The calculated Reynolds number for the pump inlet was in the laminar-to-turbulent transition area $(2300<\operatorname{Re}<4000)$ [70], which is commonly found in modeling centrifugal blood pumps [69]. Based on similar methods employed by others [53], [67], and to reduce complexity of the simulation, steady laminar flow was simulated in all cases.

The pump geometry was meshed using the structured Cartesian immersed-body method that is unique to SolidWorks Flow Simulation. This method differs from the traditional body-fitted meshing methods in that solid and fluid areas can exist within the same mesh cell, which helps minimize the overall mesh cell count and reduces computational time. A mesh independence analysis was conducted for each pump design (V1, V2) using four different meshing schemes with increasing levels of cell refinement at $Q=3.5 \mathrm{~L} / \mathrm{min}$ and $\mathrm{n}=4255$ RPM. Convergence goals $\triangle \mathrm{P}$ (generated pressure) and impeller torque were 
used to evaluate the strength of each meshing scheme (Appendix B, Table B-2 and Figures B-1, B-2). Meshing Scheme 3 showed less than a $1 \%$ difference in converged $\Delta P$ value and less than a $3 \%$ difference in converged torque value compared to the much denser meshing Scheme 4 for both Pump V1 and V2. Therefore, meshing Scheme 3 was used for V1 (484,712 cells) and V2 (317,965 cells) as a balanced compromise between calculation accuracy and mesh simplicity. All cases were run as time-dependent, transient simulations. Average inlet and outlet pressures and velocity as well as average torque (in the rotating axis) of the impeller were used as convergence goals, with a convergence metric of $2 \%$ acceptance criteria. All simulations used a variable time-step to a physical flow time of 0.25 seconds or until all goals were sufficiently converged.

\subsection{Results}

\subsubsection{Impeller Dimensions}

The core components of the Inspired Pediatric VAD include the pump housing with inlet and outlet ports, rotor body and magnet, and the impeller mounted on top of the rotor body. The impeller/rotor body assembly is magnetically levitated, rotates within the pump housing, and is designed to protect against contact of the impeller with the housing or external motor. The initial design for the Pediatric VAD impeller (Pump V1) employs a 6-blade impeller with backward swept blades and an $8 \mathrm{~mm}$ eye diameter (impeller center hole). A central opening was added to the middle of the rotor body, below the impeller, to act as a secondary blood flow path for washing of the rotor and 
impeller surfaces to help reduce thrombus formation. The central opening and secondary flow path also serve to counteract lift forces generated by the thrust of the impeller during rotation to keep the impeller/rotor body assembly centered inside the pump housing and prevent axial contact. A shroud was added to the top of the impeller blades to improve hydraulic efficiency. A summary of calculated impeller dimensions is listed in Table 3-4. The dimensions for Pump V2 are identical to V1 (Table 3-4), except for increased blade height (Blade Inlet Height $b_{1}=4.00 \mathrm{~mm}$, Blade Outlet Height $b_{2}=2.00 \mathrm{~mm}$ ). The height of the blades was increased based upon initial CFD findings that demonstrated Pump V1 failed to achieve desired performance metrics (Table 3-5). A center post in the bottom of the pump housing was added to Pump V2, which consumes much of the volume of the center opening through the rotor body to create a more uniform flow path, and reduces the priming volume from $9.5 \mathrm{~mL}(\mathrm{~V} 1)$ to $9.0 \mathrm{~mL}$ (V2). Blade tip extensions $(2.5 \mathrm{~mm})$ were also added to Pump V2 to increase the achievable pressure. Comparisons of the three-dimensional solid models of the impellers and pump housings for Pump V1 and Pump V2 are shown in Figure 3-3. 
Table 3-3. All critical calculated impeller dimensions for Pump V1.

\begin{tabular}{|c|c|c|c|}
\hline \multicolumn{4}{|c|}{ Pump V1 - Calculated Impeller Dimensions } \\
\hline Parameter & Symbol & Units & Value \\
\hline Impeller Inner/Eye diameter & $\mathrm{D}_{1}$ & $\mathrm{~mm}$ & 8.00 \\
\hline Impeller Outer Diameter & $\mathrm{D}_{2}$ & $\mathrm{~mm}$ & 27.40 \\
\hline $\begin{array}{l}\text { Estimated Rotational Speed at Design } \\
\text { Point }\end{array}$ & $\mathrm{N}$ & RPM & 4255.00 \\
\hline Rotor Height & $\mathrm{H}_{1}$ & $\mathrm{~mm}$ & 12.00 \\
\hline Blade Inlet Angle & $\beta_{1}^{\prime}$ & $\circ$ & 40.00 \\
\hline Blade Outlet Angle & $\beta_{2}$ & $\circ$ & 22.50 \\
\hline Blade Inlet Height & $b_{1}$ & $\mathrm{~mm}$ & 2.00 \\
\hline Blade Outlet Height & $b_{2}$ & $\mathrm{~mm}$ & 1.00 \\
\hline Blade Thickness & $\mathrm{S}_{\mathrm{u}}$ & $\mathrm{mm}$ & 1.00 \\
\hline Estimated Blade Number & Z & blades & 6.00 \\
\hline Blade Mean Curve Radius & $\mathrm{R}$ & $\mathrm{mm}$ & 8.92 \\
\hline Impeller Head Coefficient & $\psi$ & & 0.52 \\
\hline Impeller Flow Coefficient & $\varphi$ & & 0.12 \\
\hline Speed Number & $\sigma$ & & 0.12 \\
\hline Diameter Number & $\delta$ & & 7.99 \\
\hline Specific Speed & $\eta_{s}$ & & 1019.49 \\
\hline Volute Throat Area & $A_{\text {th }}$ & $\mathrm{mm}^{2}$ & 25.45 \\
\hline Volute Base Circle & $\mathrm{D}_{3}$ & $\mathrm{~mm}$ & 29.92 \\
\hline
\end{tabular}



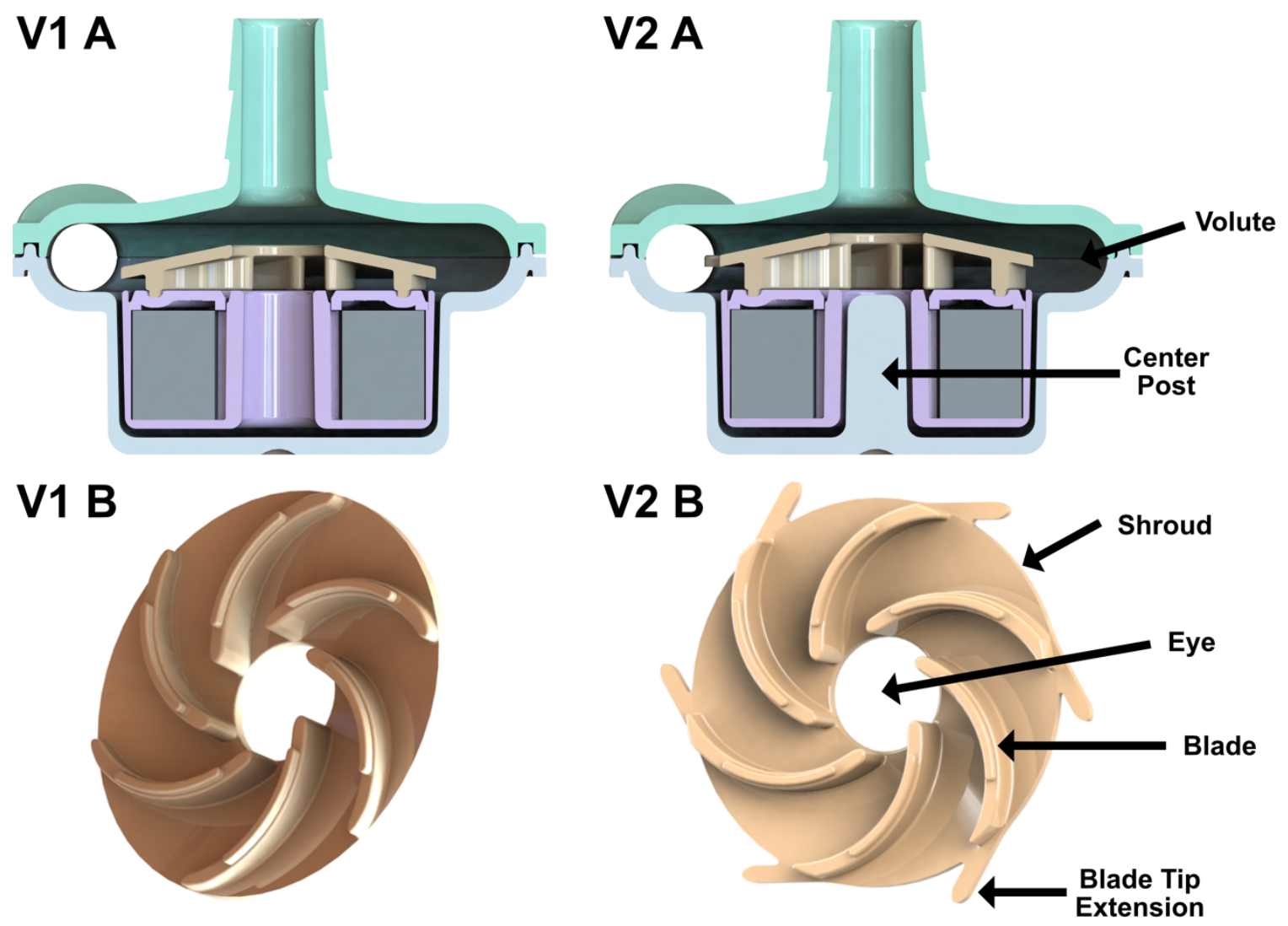

Figure 3-3. Cross section views of the 3D geometry of Pump V1 (V1 A) compared to Pump V2 (V2 A). View of the 3D geometry of the impeller for Pump V1 (V1 B) and Pump V2 (V2 B), looking from the bottom upward to see detail of the blades beneath the shroud. Highlighted impeller and pump features include the shroud, inlet eye, blades and Pump V2 added features blade tip extensions and center post.

\subsubsection{Pump Performance}

Pump V1 and $\mathrm{V} 2$ designs were unable to achieve the target flow rate $(\mathrm{Q}=$ 3.5 L/min $)$ at the target pressure $(\Delta \mathrm{P}=150 \mathrm{mmHg})$ for the estimated rotational speed $(n=4255$ RPM); However, both pump designs were able to reach the desired pressure at higher rotational speeds. Preliminary analysis showed that Pumps $\mathrm{V} 1$ and $\mathrm{V} 2$ achieved a $\Delta \mathrm{P}$ of $91 \mathrm{mmHg}$ and $120 \mathrm{mmHg}$ respectively, at $\mathrm{n}=$ 
4255 RPM (Table 3-5). Pump V2 achieved an increase of 30\% in pressure compared to Pump V1 at the simulation point with a slight increase in overall hydraulic efficiency $(\sim 1.5 \%)$.

Table 3-4. Summary of converged values pressure $(\Delta P)$ and torque and calculated hydraulic efficiency $\left(\eta_{H}\right)$ for Pumps $V 1$ and $V 2$ at $Q=3.5$ $L /$ min and $n=4255$ RPM.

\begin{tabular}{|cccc|}
\cline { 2 - 4 } \multicolumn{1}{c|}{} & $\Delta \mathrm{P}(\mathrm{mmHg})$ & Torque $(\mathrm{N}-\mathrm{mm})$ & $\begin{array}{c}\eta_{H} \\
\text { Hydraulic Efficiency }(\%)\end{array}$ \\
\hline Pump V1 & 90.80 & 5.79 & 27.37 \\
Pump V2 & 119.98 & 7.38 & 28.38 \\
\hline
\end{tabular}

\subsubsection{Pump Pressure Distribution}

Pressure distributions for pumps $\mathrm{V} 1$ and $\mathrm{V} 2$ at the simulation point, $\mathrm{Q}=$ 3.5 L/min and $n=4255$ RPM are shown in Figure 3-4 for planar cut through the center of the pump inflow (V1A, V2A), planar cut through the impeller blades (V1B, V2B), and three-dimensional isometric views of the pressure distribution on the outer surfaces of the pump housings (V1C, V2C). The static pressure at the outlet held steady at $150 \mathrm{mmHg}$ for both simulations. Pump V2 achieved lower pressures at the pump inlet and generated a larger pressure $(\Delta P)$ compared to Pump V1. 


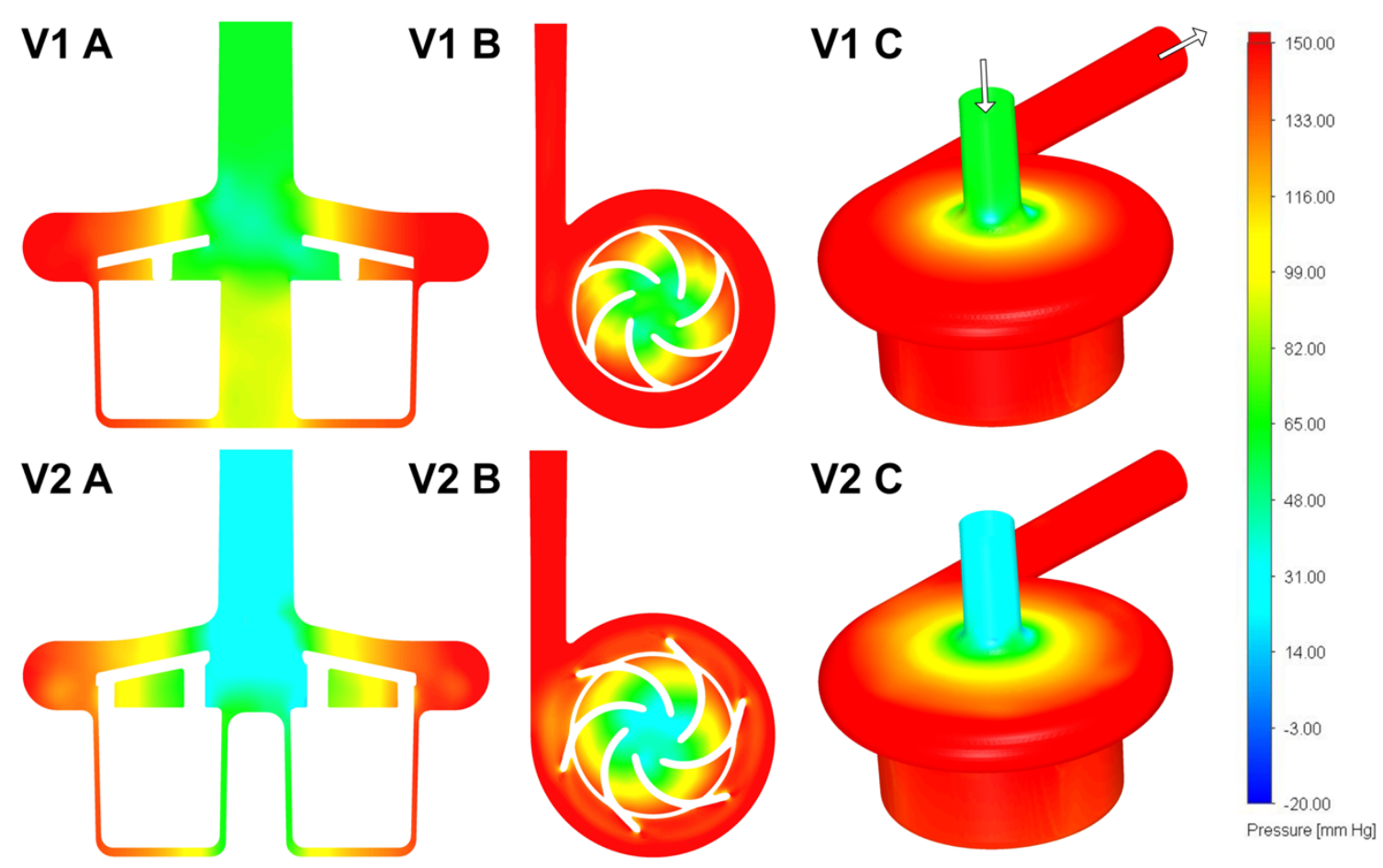

Figure 3-4. For $Q=3.5 \mathrm{~L} / \mathrm{min}$ and $n=4255 \mathrm{RPM}$, (V1 A) Pressure distribution planar to inlet center of V1, (V1 B) Pressure distribution through impeller blades of V1, (V1 C) Isometric view of pressure distribution at outer housing surfaces of V1, (V2 A) Pressure distribution planar to inlet center of V2, (V2 B) Pressure distribution through impeller blades of V2, (V2 C) Isometric view of pressure distribution at outer housing surfaces of $\mathrm{V} 2$.

\subsubsection{Pump Flow Distribution}

The velocity flow fields for Pumps V1 and V2 at the simulation point are shown in Figure 3-5 with planar cut view through the middle of the blades (V1A) and through the middle of the impeller blade extensions (V2A). Pump V1 failed to create a uniform flow field within the pump volute and also experienced flow separation at the transition of the volute to the pump outlet (also called the cutwater). Pump V2 created a relatively uniform flow distribution within the volute 
likely due to the blade extensions and increased blade height. Three-dimensional velocity flow fields are shown at a planar cut through the center of the pump inflow (V1B, V2B). In both pumps, the flow enters the eye of the impeller uniformly before turning perpendicularly into the passages between the blades. No significant vortices were observed in the blade passages for either Pump V1 or V2. There are three distinct flow paths for Pumps V1 and Pump V2: (1) a primary path where flow enters the impeller eye, travels through the blades and into the volute, and exits at the pump outlet (Figure 3-5 V1B, shown with white arrows); (2) a secondary flow path down around the outside of the rotor housing and then up through the center opening of the rotor housing (Figure 3-5 V1 B, shown with grey arrows); and (3) secondary flow at the top of the impeller shroud and the top of the pump housing where flow reverses and travels back toward the jet flow at the pump inlet (Figure 3-5 V1 B, shown with purple arrows). The secondary flow path at the top of the pump housing is minimal for Pumps V1 and V2 and only occurs close to the pump housing surface. The secondary path around the rotor body is quite different for Pump V1 compared to V2 due to the addition of the center post in V2. In Pump V1, there is strong flow close to the inner surface of the rotor body center opening, which provides good surface washing, but the flow concentrated in the middle of the center opening is random and small vortices with slow velocity fields exist. With the addition of the center 


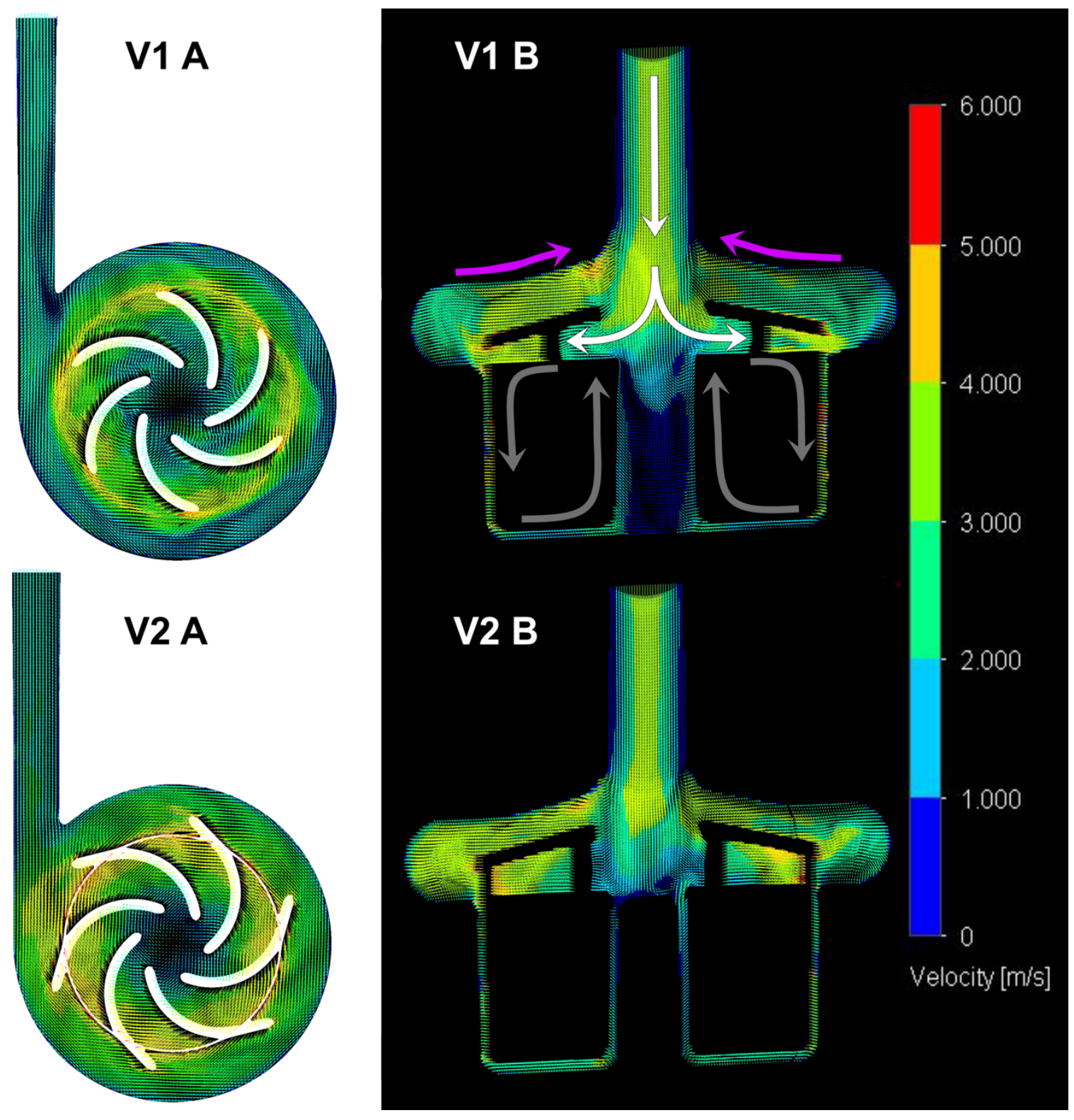

Figure 3-5. For $Q=3.5 \mathrm{~L} / \mathrm{min}$ and $n=4255 \mathrm{RPM},(\mathrm{V} 1 \mathrm{~A})$ Velocity flow fields at a planar surface cut through the center of the impeller blades for Pump V1, (V2 A) velocity flow fields at a planar surface cut through the center of the impeller blades extensions for Pump V2, (V1 B) three dimensional velocity flow fields planar to inlet center for Pump $V 1$ with highlighted flow paths; primary (white arrows), rotor secondary (grey arrows), pump housing secondary (purple arrows), (V2 B) three dimensional velocity flow fields planar to inlet center for Pump V2. 
post in Pump V2, and the tight $0.5 \mathrm{~mm}$ gap between the rotor body and the center post, the flow velocity is strong along both the rotor body and center post surfaces.

\subsubsection{Wall Shear Stress}

Wall shear stress (WSS) surface plots for the pump impeller and pump housing surfaces are shown in Figure 3-6 for pumps V1 and V2 at the simulation point, $\mathrm{Q}=3.5 \mathrm{~L} / \mathrm{min}$ and $\mathrm{n}=4255 \mathrm{RPM}$. Areas on the surfaces that experience WSS greater than $150 \mathrm{~Pa}$ are highlighted in red. Shear stresses between $150-$ $250 \mathrm{~Pa}$ with flow exposure times of 102 seconds have been shown to cause hemolysis of red blood cells [71]. The highlighted areas for both Pump V1 and V2 are minimal but there are still some unavoidable concentration points, especially at the impeller blade exit and impeller blade extensions, that should be investigated further in future hemolysis studies. Additionally, all though the center post found in Pump V2 greatly improves the velocity flow field of the blood in this region, it appears to cause a concentration of high shear stress not found on Pump V1. This will be investigated further in future studies. Mean WSS values for both Pump V1 and V2 can be found in Appendix B, Table B-3.

\subsubsection{Increased Rotational Speed}

CFD simulations were also conducted for Pumps V1 and V2 at higher rotational speeds of $4500,5000,5500$, and 6000 RPM in an attempt to achieve the design point $\Delta \mathrm{P}=150 \mathrm{mmHg}, \mathrm{Q}=3.5 \mathrm{~L} / \mathrm{min}$ with resulting pressures (Figure 


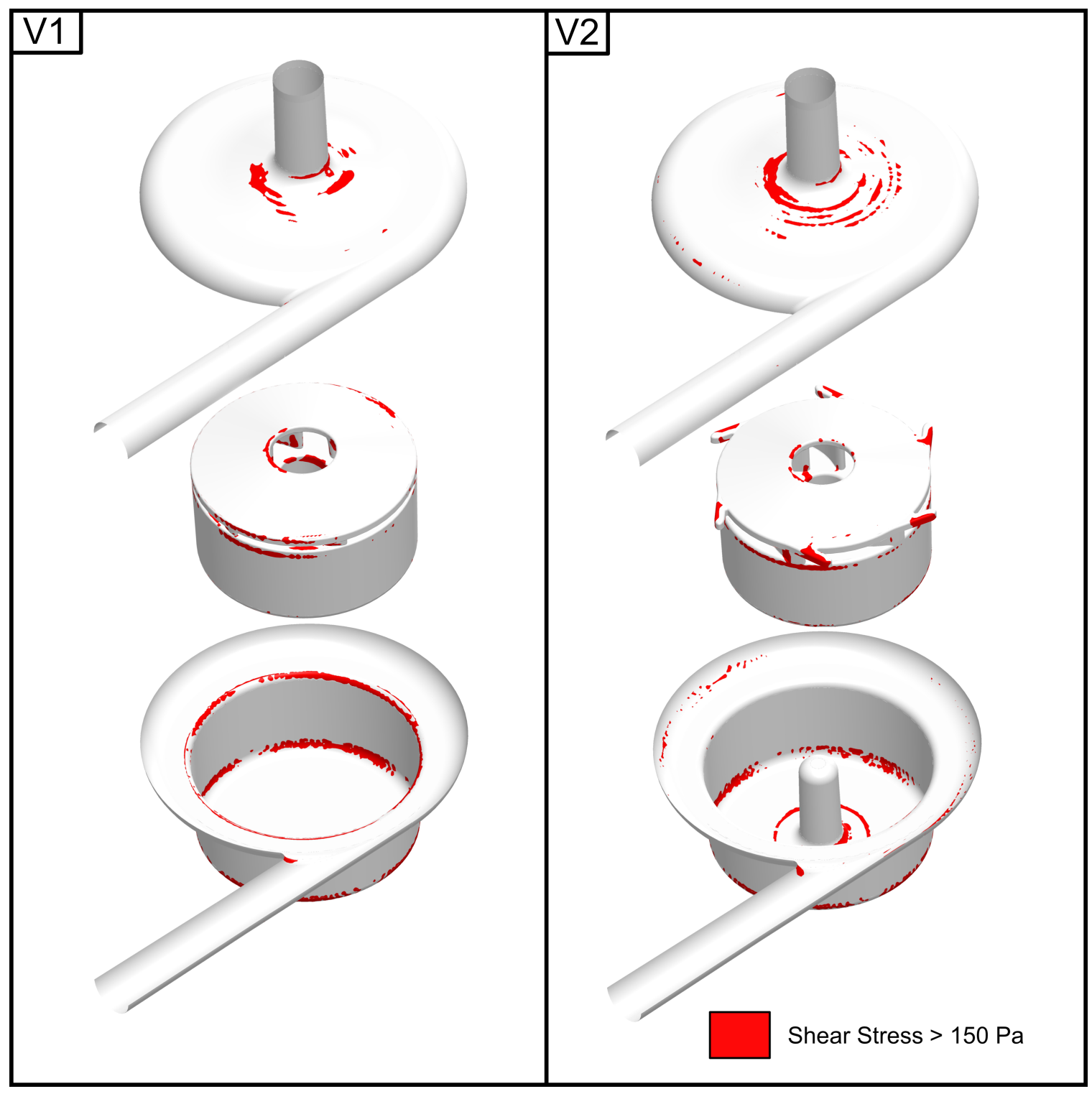

Figure 3-6. Surface plots of Pump V1 and V2 pump housings and impellers indicating areas where wall shear stress (WSS) values exceed 150 $P a$ at $Q=3.5 \mathrm{~L} / \mathrm{min}$ and $n=4255 \mathrm{RPM}$.

3-7A) and hydraulic efficiencies (Figure 3-7B) over the range of rotational speeds presented. Pump V1 was able to achieve $150 \mathrm{mmHg}$ between 5000 5500 RPM, with a maximum pressure of $230 \mathrm{mmHg}$ at 6000 RPM. Pump V2 was able to achieve $150 \mathrm{mmHg}$ between 4500 - 5000 RPM, with a maximum pressure of $279 \mathrm{mmHg}$ at $6000 \mathrm{RPM}$. Pumps V1 and V2 achieved their 
maximum efficiency point for $\mathrm{Q}=3.5 \mathrm{~L} / \mathrm{min}$ at $5000 \mathrm{RPM}$ with an efficiency of $30 \%$ for V1 and $29 \%$ for V2. Pressure distributions for Pump V2 at a planar cut through the middle of the impeller blade extensions are shown in Figure 3-8. As expected, the pressure distributions within the pump and impeller increase with higher gradients with increasing rotational speed.

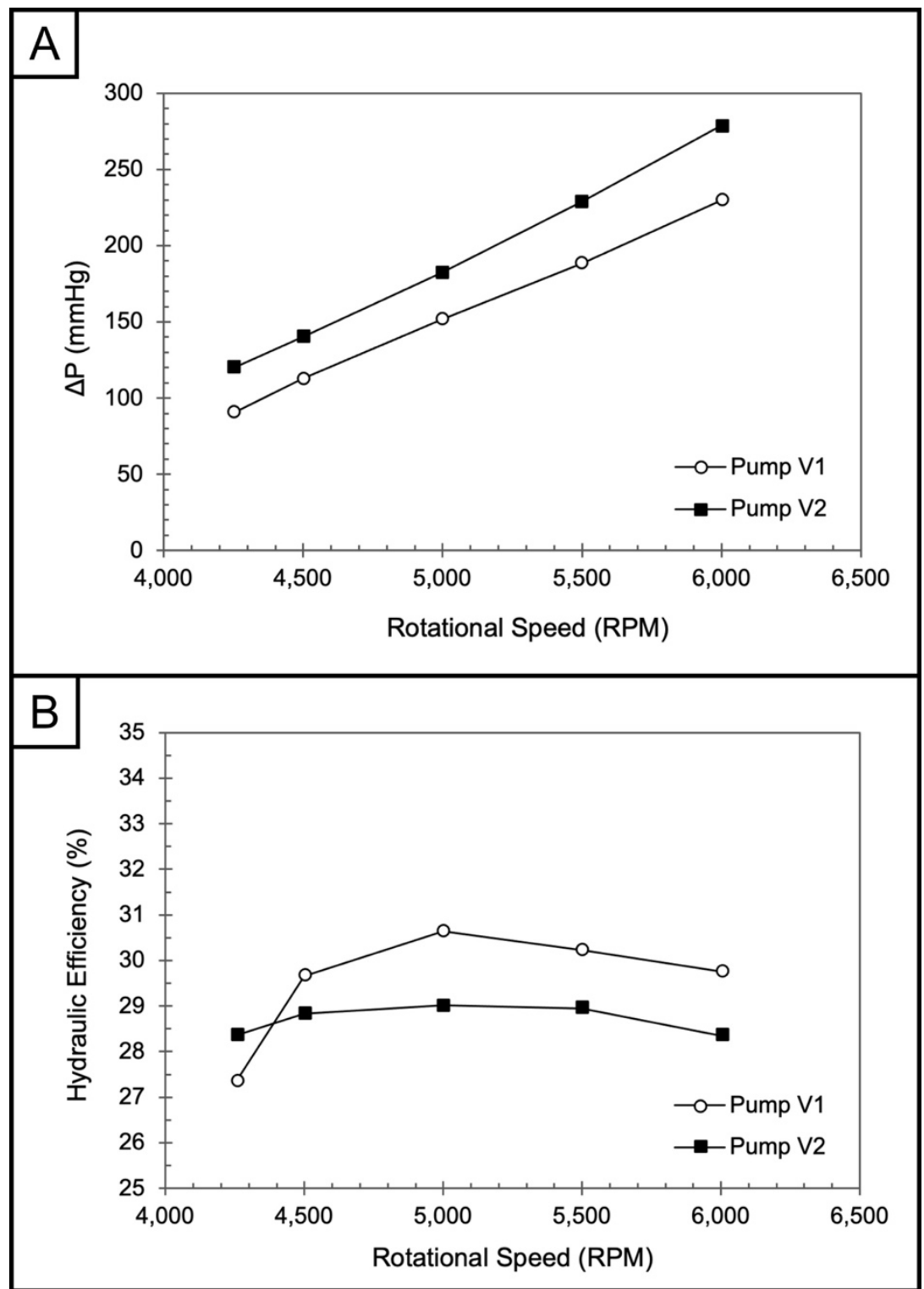

Figure 3-7. (A) Plotted generated pressure $(\Delta P)$ versus rotational speed (n) at $Q$ $=3.5 \mathrm{~L} / \mathrm{min}$ for Pumps V1 and V2, (B) Plotted calculated hydraulic efficiencies $\left(\eta_{H}\right)$ versus rotational speed $(n)$ at $Q=3.5 \mathrm{~L} / \mathrm{min}$ for Pumps V1 and V2. 

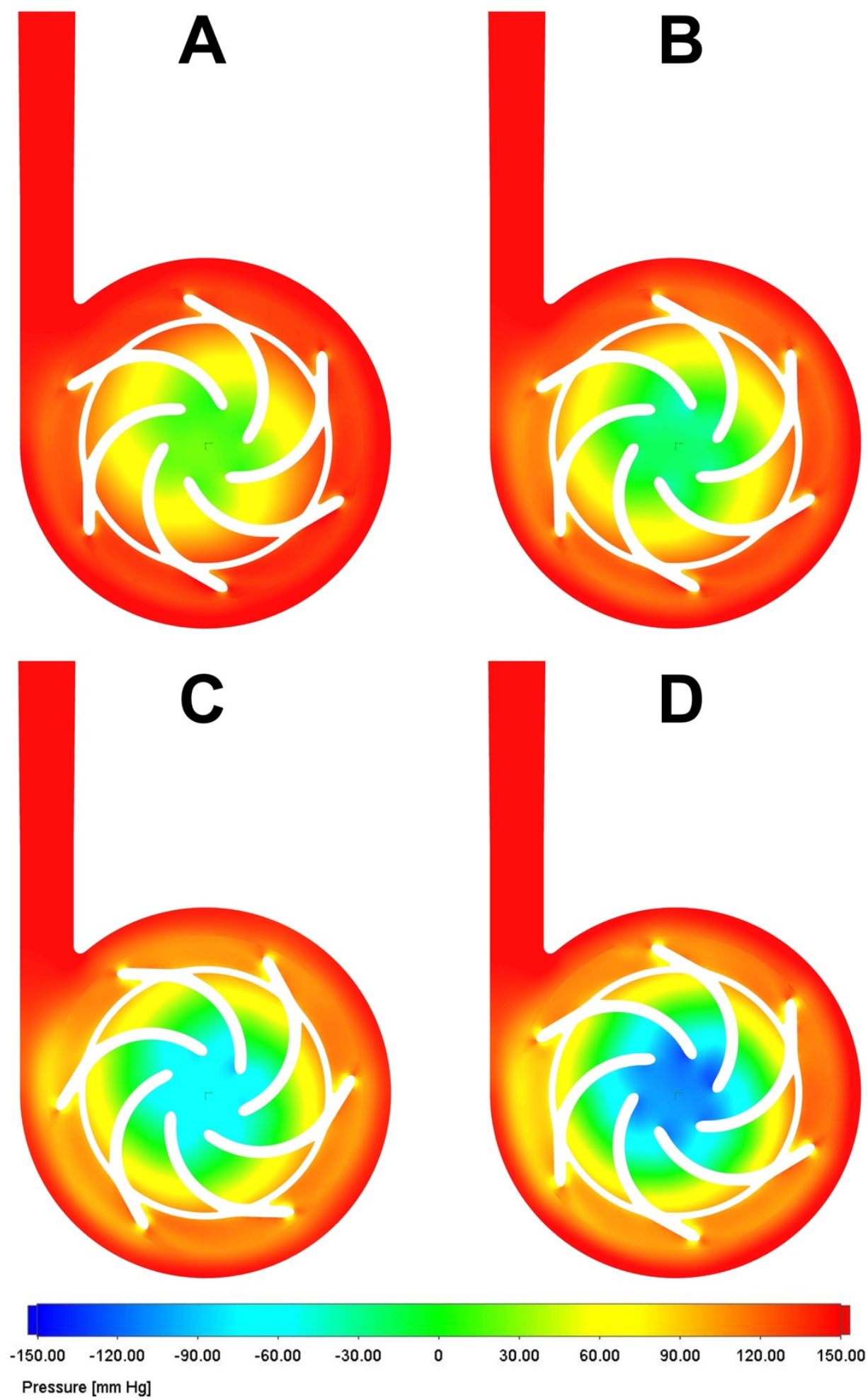

Figure 3-8. Pressure distributions at a planar surface cut through the middle of the impeller blade extensions for Pump V2 at $Q=3.5 \mathrm{~L} / \mathrm{min}$ and $(A)$ $n=4500$ RPM, (B) $n=5000$ RPM, (C) $n=5500$ RPM, (D) $n=6000$ RPM. 


\subsection{Discussion}

The Inspired Pediatric VAD was successfully designed, modeled, and virtually simulated to produce the desired peak operating conditions by achieving a flow of $3.5 \mathrm{~L} / \mathrm{min}$ at $150 \mathrm{mmHg}$. Pump V2 was able to produce higher pressures than Pump V1 (20\% to $30 \%$ increase) at all simulated rotational speeds, and the design reduced the priming volume to $9 \mathrm{~mL}$ ( $5 \%$ reduction). Pump V1 and V2 designs were able to produce clean velocity distributions through the impeller blades and relatively uniform pressure distributions at the initial rotational speed $n=4255$. Pump V1 did not produce a uniform velocity distribution within the volute, which may be attributed to the small blade outlet height $(1 \mathrm{~mm})$ in relation to the volute throat diameter $(5.7 \mathrm{~mm})$, and also experienced flow separation at the volute cut-water. Pump V1 also experienced non-uniform flow within the center opening of the rotor body at $n=4255$ RPM. The addition of the blade tip extensions, increased blade height, and a center post in Pump V2 appear to have resolved these limitations. Pumps V1 and V2 required operation at higher rotational speeds to achieve the desired pressure $(\Delta \mathrm{P}=150 \mathrm{mmHg})$.

The Inspired Pediatric VAD achieved a maximum hydraulic efficiency for $\mathrm{Q}=3.5 \mathrm{~L} / \mathrm{min}$ at $5000 \mathrm{RPM}$ for both Pump designs. Pump V1 exhibited slightly higher efficiencies compared to Pump V2 at all rotational speeds, except at 4255 RPM, even though V2 produced much higher pressures. These results were anticipated since the hydraulic efficiency is inversely proportional to the impeller torque, and Pump V2 design included impeller blade extension with larger 
momentum arm, thereby generating greater torque. The typical calculation to determine hydraulic efficiency is to divide the fluid power $(Q \times \Delta P)$ by the input power of the motor driving the impeller. For this study, impeller torque was derived from the CFD simulation by summing the torque experienced by all impeller surface faces. While this method provides an estimate of the current efficiencies, the true values will be revealed once a motor power term can be determined from physical prototypes. Simulations predict Pumps V1 and V2 will compare favorably to other VADs in the desired flow range. The Inspired Pediatric VAD may achieve $30 \%$ efficiency, which compares favorably to the DP3 at $27.5 \%(3.5 \mathrm{~L} / \mathrm{min}, 150 \mathrm{mmHg})$ [50], Rotaflow at $26 \%(3.5 \mathrm{~L} / \mathrm{min}, 150 \mathrm{mmHg})$ [50], and PediMag at 37\% (3.5 L/min, unknown pressure) efficiencies [72].

Though the initial findings from this study are promising, the CFD simulations of the pump designs only offer predictions of its potential performance. These CFD predictions must now be validated by testing a physical prototype of the pump in a mock circulatory loop utilizing a blood analogue glycerol-water solution as well as whole blood. The initial flow field predictions from the CFD in this study are also promising, but to fully characterize these, further CFD simulations need to be conducted with a more in-depth look at flow leakage and retrograde flow around the impeller (which causes hydraulic losses) as well as formation of vortices inside the impeller blade passage and at the blade exit. These new CFD studies will be validated through particle image velocimetry (PIV) experiments of a pump prototype. Lastly, the hemodynamic performance of the pump and impeller designs will need to be evaluated through 
CFD hemolysis predictions and hemolysis flow loop experiments. Evaluating the hemolysis rates in this device will be critical in determining any areas of the impeller or pump geometry that may need further design modifications.

Advanced design features of the Inspired Universal System may offer functional, operational, and technological advantages compared to other currently available extracorporeal VADs including the EXCOR, BP-50, RotaFlow, DP3, and PediMag. The EXCOR and PediMag devices represent the only options specifically designed for pediatric patients. The EXCOR requires use of a large external console and is pneumatically driven. The system operates in a pulsatile mode and uses a polymeric membrane and a pair of polymer valves [47] both of which are susceptible to fatigue. The EXCOR device has been associated with limited long-term use and durability [48]. The PediMag is a miniaturized continuous flow centrifugal pump that uses MagLev technology to drive a suspended impeller. The PediMag device is only approved for low flows (1.5 L/min) and short-term use (6 hours), though it is often used for longer durations [73], and requires a large bed-side coupled motor and controller unit [74] that does not allow patients to ambulate during treatment. The BP-50, RotaFlow, and DP3 are rotary continuous flow pumps that use magnetic coupling rather than true MagLev to rotate the impeller around a physical bearing. The Inspired Pediatric VAD will employ a contactless bearing design coupled with the MagLev technology to levitate and rotate the impeller rotor. In contrast, in magnetic coupling the impeller/rotor is still anchored by a physical bearing, 
creating a concentrated point for mechanical wear, heat generation, and blood damage [17], [18].

\subsection{Conclusion}

Throughout this conceptual phase of research and development of the Inspired Universal MagLev System, the objective has been to design and evaluate a next generation extracorporeal MCS system for pediatric patients suffering from cardiopulmonary disorders. The design goal has been to develop a modular system capable of providing adequate hemodynamic support in a form factor that is smaller, more portable (wearable) and intuitive to use for caretakers responsible for managing the wellbeing of pediatric patients.

This study serves as the first of a series of articles that will describe the engineering effort to develop the Inspired System. The initial focus has been on the design phase for the Inspired Pediatric VAD with an attempt to introduce multiple novel features compared to commercially available pumps while also demonstrating that the resulting design met product development requirements. Subsequent articles will describe the fabrication and testing of the VAD prototype, design and testing of the MagLev motor, and in vitro and in vivo studies that evaluate the safety and performance of the Inspired Universal System.

The pediatric heart failure population remains in dire need of a dedicated MCS solution, for which the entire HF community may benefit from a compact, low-cost MCS option that can be adapted to fit individual patient needs. Our goal 
is to produce future single use, 30 -day modules to provide respiratory assistance with a potential module that will include an integrated pump/oxygenator that could greatly reduce the size of a traditional ECMO circuit. The hope will be to design the system to allow a patient suffering from severe respiratory dysfunction to be ambulatory. If this system were available today with the COVID-19 pandemic, there would likely be widespread adoption of the technology. In summary, the preliminary hydrodynamic data generated thus far for the Inspired Pediatric VAD have been encouraging. The next phase of development will focus on characterization, human factors engineering, and verification that the Inspired System performs as designed. 


\section{CHAPTER 4 : DEVELOPMENT OF INSPIRED THERAPEUTICS PEDIATRIC \\ VAD: QUANTIFYING IMPELLER TORQUES AND FORCES FOR MAGLEV MOTOR DESIGN}

\subsection{Preface}

Pediatric heart failure (HF) patients have historically been an underserved population and have the highest mortality rate at $17 \%$ of any population awaiting heart transplantation [11]. Due to this great clinical need, Inspired Therapeutics (Merritt Island, FL) and the University of Louisville (Louisville, KY) have been developing the Inspired Pediatric VAD, a rotary blood pump to be used with the Inspired Universal MagLev System. As seen in Table 3-2, the vision for this system is a universal controller/motor which can be paired with a variety of different single-use pumps for different indications for use (neonate, pediatric and

Authors: ${ }^{1}$ Landon H. Tompkins, MEng., ${ }^{2}$ Steven R. Prina Ph.D., ${ }^{3}$ Barry N. Gellman M.S., ${ }^{4}$ Gino F. Morello, , ${ }^{1}$ Thomas Roussel Ph.D., ${ }^{1}$ Jonathan A. Kopechek Ph.D., ${ }^{5}$ Stuart J. Williams Ph.D., ${ }^{3}$ Priscilla C. Petit, ${ }^{6}$ Mark S. Slaughter M.D., ${ }^{1,6}$ Steven C. Koenig Ph.D., ${ }^{1,3,6}$ Kurt A. Dasse Ph.D.

Department of Bioengineering, University of Louisville, Louisville, $K Y$

BLDC Designs LLC, Rocklin, CA

Inspired Therapeutics LLC, Merritt Island, FL

Veritium Research LLC, Fort Lee, NJ

Department of Mechanical Engineering, University of Louisville, Louisville, KY

Department of Cardiovascular and Thoracic Surgery, University of Louisville, Louisville, $K Y$ 
adult) providing up to 30 -days of circulatory or respiratory support. The development goals for the system are to produce a product that is smaller, less expensive, and wearable to allow for patient ambulation during treatment. The impeller and pump design concepts for the Pediatric VAD have been previously described by the authors [22]. The next phase in the development process focuses on the design of the novel MagLev motor that will be integrated into the Universal MagLev System to levitate and rotate the Pediatric VAD impeller/rotor. This article describes the methodology and presents the results of computational fluid dynamics (CFD) model simulations and in vitro testing (static mock flow loop model) to characterize pump pressure and flow performance and hydraulic torques and translational forces acting on the pump impeller/motor. These results will be used to complete the design of the novel MagLev motor.

\subsection{Introduction}

Motor designs for rotary blood pumps (RBP) have many unique requirements. Not only do these motors need to be compact and demonstrate long-term reliability, they also require relatively large gaps between rotor and stator components (compared to traditional motors) to accommodate channels for adequate blood flow and the use of contactless bearings to prevent blood damage [75]. Modern RBPs typically use electric motors with an external motor stator, pump housing, internal rotating element (typically a combination impeller and motor rotor), and bearings to allow for rotation and impeller/rotor stability

(Figure 4-1). The driving portion of the motor that rotates the impeller/rotor is 
achieved using a combination of permanent- and electromagnets, which may also serve to levitate the impeller/rotor within the pump casing.

To stabilize the impeller/rotor and provide a point of rotation, several RBP designs, including the Medtronic BioPump BP-50, Maquet RotaFlow, and Medos Deltastream DP3, use a shaft and mechanical bearing attached to the center of the rotor [18], [49], [51]. Although these designs use small bearings with minimal contact, they create a concentrated point for mechanical wear, heat generation, noise, vibration, and a potential risk for clinically significant blood damage [76]. Modern designs have employed contactless bearings using permanent or electromagnetics between the rotor and stator or hydrodynamic bearings as is the case of the HeartWare HVAD [77], which provide improved hemodynamics that show promise in helping to reduce the risk of blood damage [78]. A unique design used in the Thoratec HeartMate 3 and Levitronix CentriMag systems is based on a "bearingless slice motor", which combines the motor and bearing into a single component [79], [80]. Irrespective of their novel design and orientation, all rotary blood pump motors must be hemocompatible, generate sufficient torque to rotate the impeller/rotor, and provide translational forces to stabilize the rotation of the impeller/rotor. Motor designs may also be unique to each pump type, including different sized diameter impeller/rotors and operational ranges (pressures and flows) for the intended patient populations. This variance in 
geometries and operational parameters may directly impact the torques and forces the motor may be required to produce.

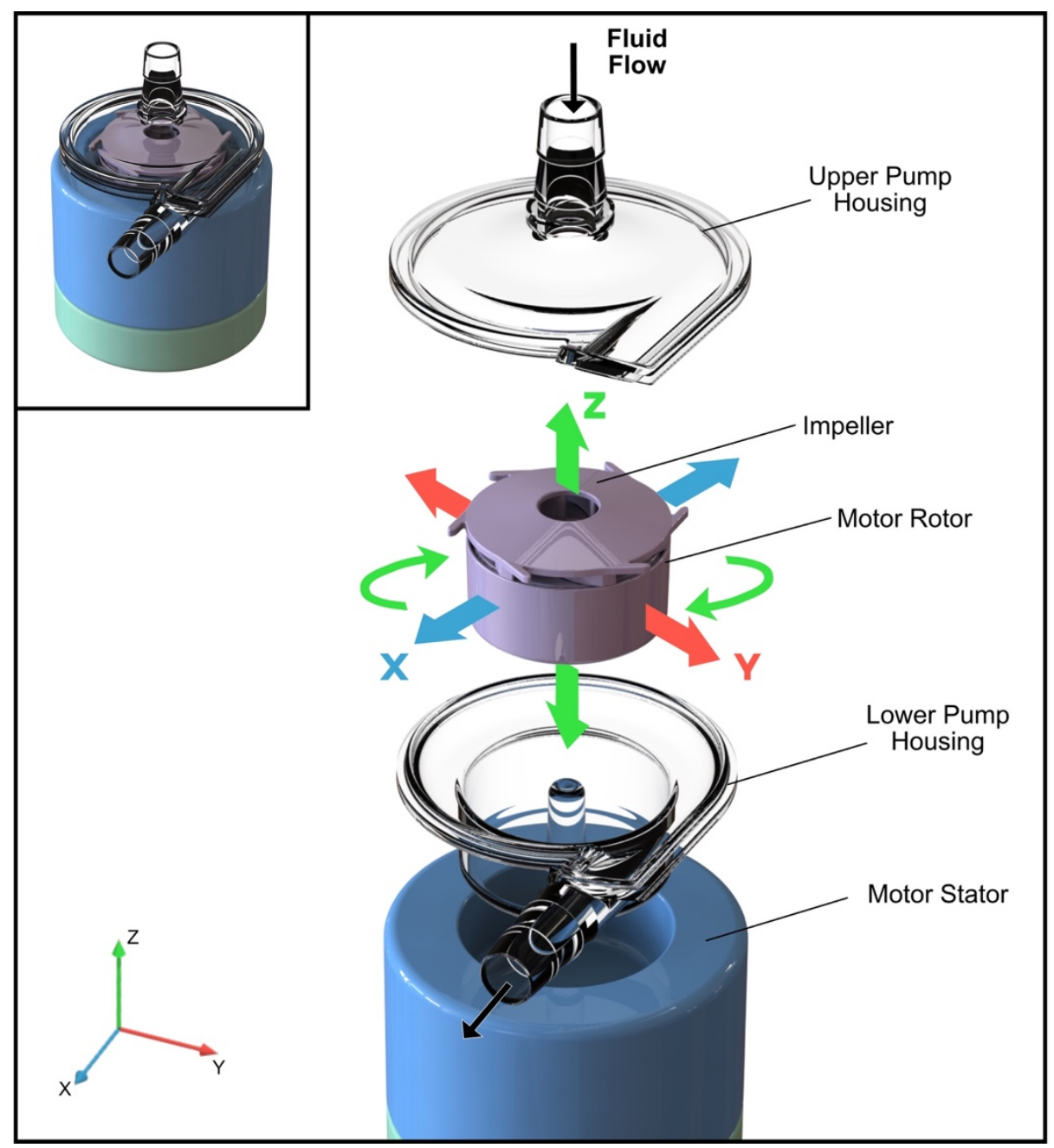

Figure 4-1. Exploded view of the Inspired Pediatric VAD concept detailing the fluid flow direction, impeller rotational direction (Z-axis), impeller translational force directions (X, Y, Z-axis) and the pump components; the Upper Pump Housing, Impeller, Motor Rotor, Lower Pump Housing, and Motor Stator.

The proposed motor design for the Inspired Pediatric VAD is a MagLev system that completely levitates and rotates the pump impeller/rotor in a manner that eliminates physical contact between parts. The impeller/rotor is levitated 
within the pump housing, providing a blood gap of $0.5 \mathrm{~mm}$ around the sides and $0.75 \mathrm{~mm}$ at the bottom of the rotor, with a total gap of approximately $2 \mathrm{~mm}$ between rotor and stator magnetic components (Figure 4-2). With these dimensions in place, the performance of the pump impeller/rotor and surrounding fluid within the pump requires characterization over the anticipated operational

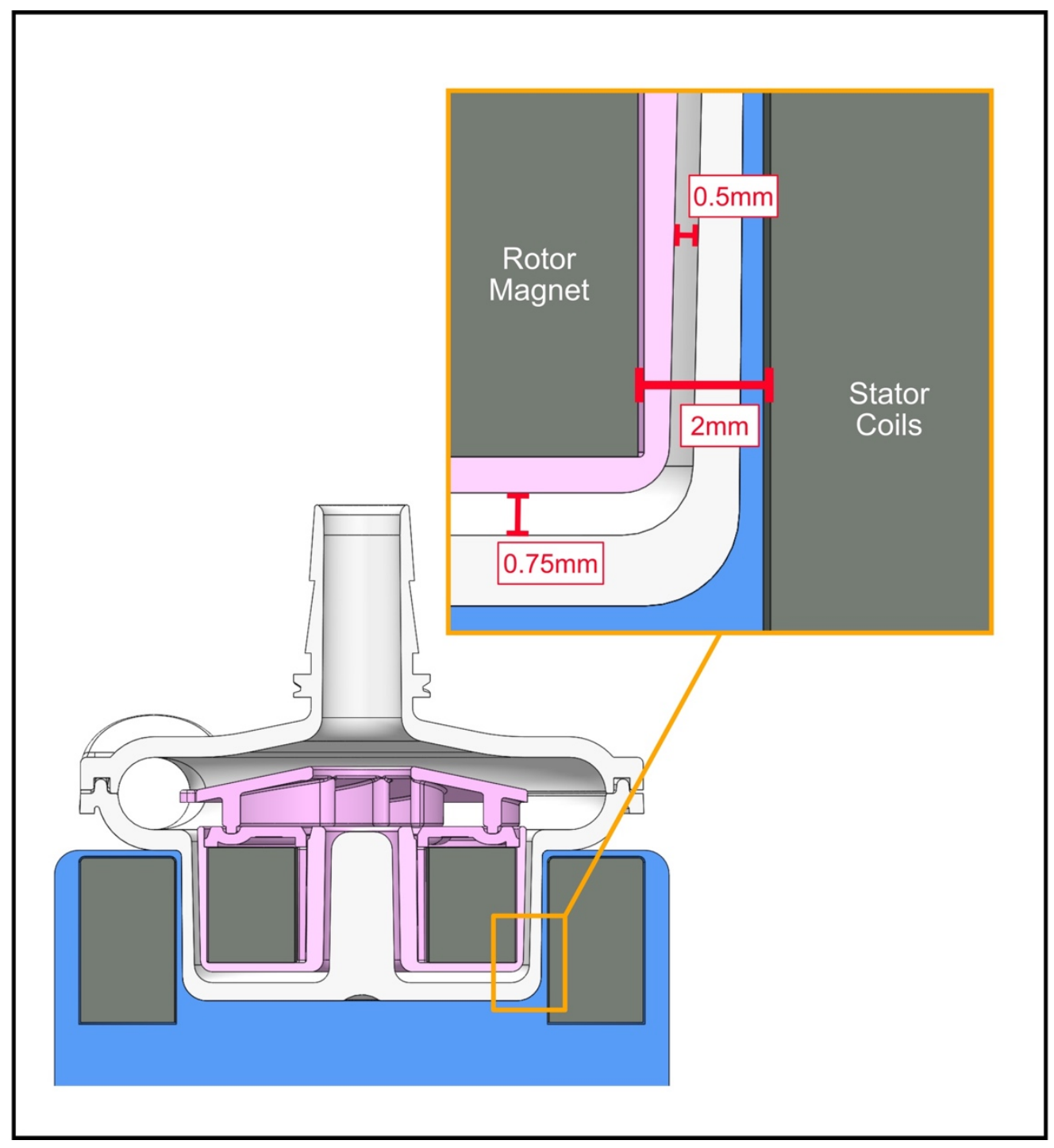

Figure 4-2. Cut-view of the Inspired Pediatric VAD concept detailing the blood/fluid gap between the impeller/rotor and pump housing $(0.5$ $\mathrm{mm}$ along sides, $0.75 \mathrm{~mm}$ along bottom) and the overall gap (2 $\mathrm{mm})$ between rotor and stator magnetic components. 
range of the pump to define the design requirements for the MagLev motor. The motor performance characteristics include the required torque to rotate the impeller and propel the fluid (rotation about the Z-axis), the axial lift force (translation in the Z-axis) generated by the impeller acting on the fluid, and the hydrodynamic forces from the surrounding fluid acting on the impeller/rotor in the tangential, radial, and axial directions ( $\mathrm{X}, \mathrm{Y}$, and Z-axis, respectively) (Figure 41). In this article, we present in vitro testing of an Inspired Pediatric VAD pump and the CFD model simulations used to quantify and define these critical system design parameters.

\subsection{Methods}

\subsubsection{Prototype Construction}

A prototype of the Inspired Pediatric VAD was constructed to quantify the required magnitude and range of impeller hydraulic torques and to benchmark hydrodynamic performance. This test device was assembled using a shaft to rotate the pump impeller in place of the future MagLev motor and bench tested in a static flow loop (SFL, Figure 4-3 A,B,C). This simplified shaft driven prototype allowed testing of critical parameters (pump performance, impeller torque) which will translate into engineering requirements to complete the MagLev motor design. The lower pump housing, impeller, and upper pump housing were fabricated using additive manufacturing techniques that meet design specifications in accordance with the defined geometries calculated using computational models, as previously described [22]. The components were 
printed using stereolithography with a proprietary high-resolution ABS-like clear material (WaterShed XC). The impeller was fabricated without a center opening in the rotor body to allow coupling of an $8 \mathrm{~mm}$ diameter shaft to be driven by a permanent magnet DC motor. The pump assembly was mounted on a custom fixture with the DC motor at the base. A nitrile rubber double-lip seal was added to the lower pump housing as a leak-proof entry for the DC motor shaft. The shaft was permanently attached to the center of the impeller/rotor and coupled to the

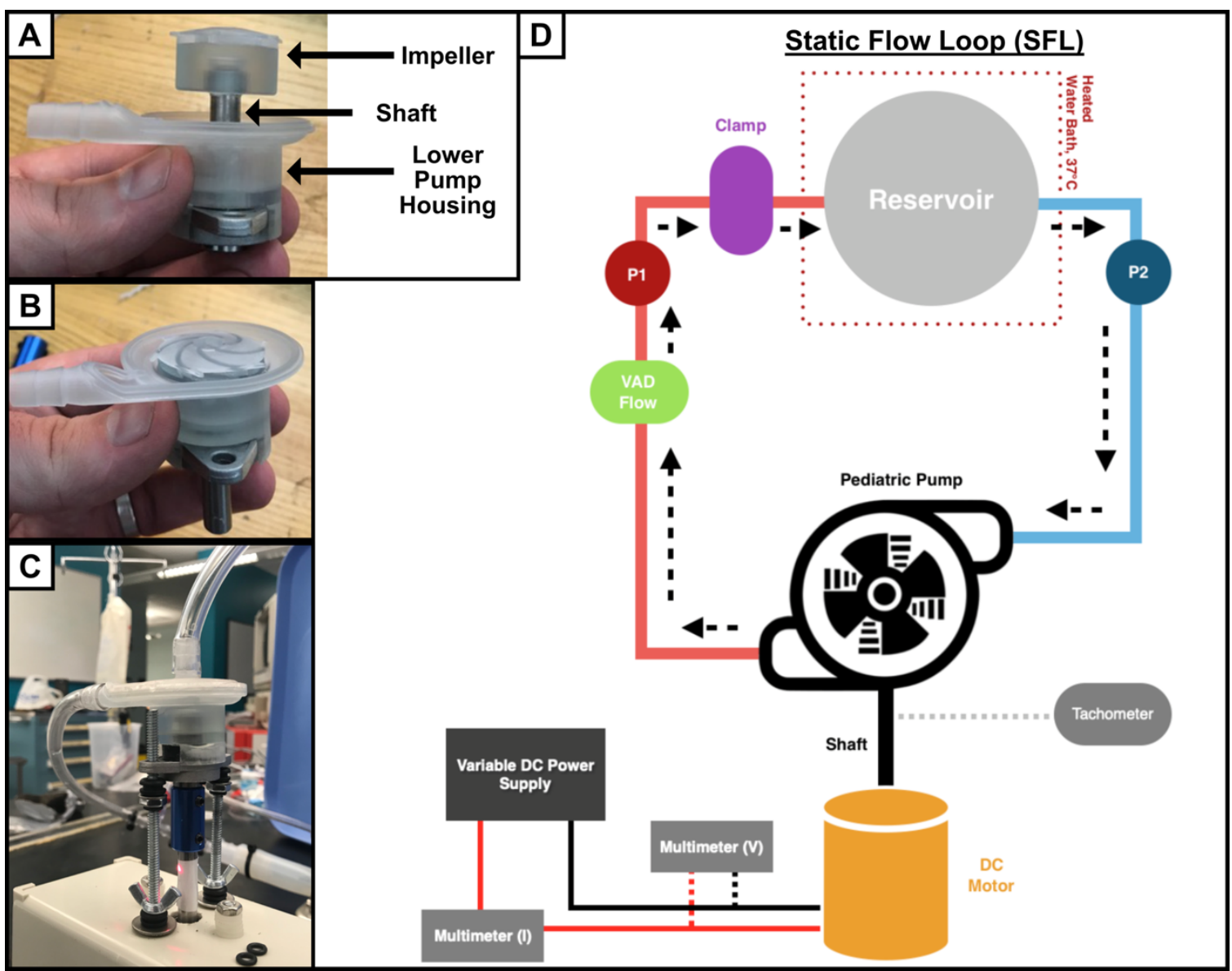

Figure 4-3. (A) Side and (B) Top views of the prototype of the Inspired Pediatric VAD including SLA polymer impeller, lower pump housing, and $8 \mathrm{~mm}$ metal shaft. (C) VAD prototype attached to custom fixture with coupled DC motor and tachometer, which are connected to the (D) static flow loop (SFL) for preliminary performance testing and evaluation. 
DC motor shaft via a mechanical coupler. This attachment mechanism was also used to set the height of the impeller within the pump housing to maintain the fluid/blood gaps between the impeller and housing. A laser-based tachometer (PLT200, Monarch Instrument, Amherst, NH) was used to measure the rotational speed of the shaft and impeller.

\subsubsection{Determination of Impeller Hydraulic Torque}

A permanent magnet DC (PMDC) motor (12V/24V 3000/6000rpm, Guang Wan Motor Co, LTD.) powered by a variable DC power supply (PS280, Tektronix, Beaverton, OR) was used to rotate the VAD impeller. The motor current and supply voltage were measured with a pair of digital multimeters (Fluke 45, Fluke Corporation, Everett, WA). Additionally, this motor was used to determine the magnitude of hydraulic torque, $\boldsymbol{\tau}_{\text {Hydro, }}$ (torque due to circulating fluid) acting on the impeller over a range of rotational speeds (500 - 6000 RPM) and pressures $(\Delta \mathrm{P}, 0$ to $\sim 420 \mathrm{mmHg})$ using the following protocol. First, an average implied motor torque constant $\left(\mathrm{K}_{\mathrm{t}}\right)$ was determined for the motor to calculate the torque ( $\tau$ ) based on motor current (I), (Equation 4.1) [81].

$$
\tau=K_{t} \times I
$$

This measurement was obtained using another DC motor to back-drive the PMDC motor over a range of rotational speeds (400 to 7500 RPM). At each speed, the voltage generated by the motor was recorded (Appendix C, Table C- 
1), and an average back electromotive force (EMF) constant for the motor was determined ( $\mathrm{K}_{\mathrm{e}}$, Equation 4.2). By converting the units, the average back EMF constant is equivalent to an implied motor torque constant $\mathrm{K}_{\mathrm{t}}$ (Equations $4.3,4.4$ ) [81].

$$
\begin{gathered}
K_{e}=\text { Voltage }(V) / \text { Rotational Speed }(\omega) \\
K_{t}=\text { Motor Torque }\left(\tau_{D C}\right) / \text { Motor Current }(I) \\
K_{e}=K_{t}
\end{gathered}
$$

The calculated values were $\mathrm{K}_{\mathrm{e}}=3.311 \mathrm{~V} / \mathrm{kRPM}$ and $\mathrm{K}_{\mathrm{t}}=31.57 \mathrm{~N}^{*} \mathrm{~mm} / \mathrm{A}$. The PMDC motor was then operated using the variable power supply over the range of rotational speeds against no load. Motor voltage and current were recorded at each rotational speed in 500 RPM increments. The total torque $\left(\boldsymbol{\tau}_{\mathrm{DC}}\right)$ generated by the DC motor alone was then calculated at each rotational speed (Appendix C, Table C-2) defined as the product of the motor current (I) and $\mathrm{K}_{\mathrm{t}}$. The calculated torque was plotted against rotational speed (Appendix C, Figure C-1), and a corresponding linear curve of best fit was obtained. The associated linear equation (Equation 4.5) $\left(R^{2}=0.9839\right)$ was used to generate a secondary equation to calculate the total torque of the $\mathrm{DC}$ motor $\left(\boldsymbol{\tau}_{\mathrm{DC}}\right)$ at any rotational speed $(\omega)$, including the motor viscous damping coefficient $\left(B_{m}\right)$ and the kinetic friction of the motor $\left(\mathrm{T}_{\mathrm{fm}}\right)[82]$ : 


$$
y=0.7948 x+6.8301 \rightarrow \tau_{D C}=\left(B_{m} \times \omega\right)+T_{f m}
$$

The total PMDC motor torque represents the base torque of the system, and any generated torque above PMDC motor torque would be due to friction losses from the shaft and pump housing seal and hydraulic forces acting against the impeller. To determine the torque losses due to friction of the shaft and seal $\left(\boldsymbol{\tau}_{\text {Friction }}\right)$, the PMDC motor was coupled to the Pediatric VAD impeller through the center shaft and placed on the testing rig. The Pediatric VAD was not filled with fluid. The PMDC motor was operated over a range of rotational speeds (1000 RPM increments) and the motor voltage and current were recorded at each speed to calculate the total torque of the dry system ( $\left.\boldsymbol{\tau}_{\text {Total, Dry }}\right)$ (Appendix $\mathbf{C}$, Table C-3). The average torque due to friction and other losses ( $\left.\boldsymbol{\tau}_{\text {Friction }}\right)$ of the shaft and seal was calculated based on the difference between the total torque of the dry system ( $\left.\boldsymbol{\tau}_{\text {Total, Dry }}\right)$ and the total torque of the DC motor $\left(\boldsymbol{\tau}_{\mathrm{DC}}\right)$, (Equation 4.6).

$$
\tau_{\text {Friction }}=\tau_{\text {Total,Dry }}-\tau_{D C}
$$

The total torque ( $\left.\boldsymbol{\tau}_{\text {Total }}\right)$ and impeller hydraulic torque $\left(\boldsymbol{\tau}_{\text {Hydro }}\right)$ of the pump were calculated using results from the static flow loop model. 


\subsubsection{Static Flow Loop (SFL) Model Testing}

A static flow loop (SFL) model consisting of a fluid reservoir, compliant silicone tubing, and a screw-type clamp (to adjust flow resistance) was constructed (Figure 4-3D). The fluid reservoir was submerged in a heated water bath with a thermoregulator unit (Techne TE-10D, Cole-Parmer, Vernon Hills, IL) to maintain temperature at $37^{\circ} \mathrm{C}$. The Inspired Pediatric VAD was inserted in-line with the SFL circulation. High-fidelity pressure transducers (MPR-500, Millar, Houston, TX) were placed at the pump inlet (P2) and outlet (P1), and a transittime flow probe (6XL, Transonic, Ithaca, NY) at the pump outlet (VAD Flow). The SFL was filled with one liter of a blood analogue solution consisting of a salineglycerol mixture with $3.6 \mathrm{cP}$ viscosity (equivalent to the viscosity of $38 \%$ hematocrit blood [83], [84]). A $10 \mathrm{ml}$ sample of the solution was tested using a calibrated Cannon-Fenske style viscometer submerged in a $37^{\circ} \mathrm{C}$ water bath to quantify the measured density of the sample $(1.1096 \mathrm{~g} / \mathrm{ml}$ with a viscosity of 3.66 $\mathrm{cP})$.

The hydrodynamic performance of the Pediatric VAD was tested across the range of rotational speeds (500 - 6000 RPM) via the following protocol. The voltage of the DC power supply was adjusted to produce a VAD impeller rotational speed starting at 500 RPM, confirmed by the tachometer. With the resistance clamp completely open (no load), the pump inlet and outlet pressures, flow rate, and the motor voltage and current were measured at the set rotational speed. This condition provided the maximum flow rate of the pump against minimum resistance $(\Delta \mathrm{P}=0 \mathrm{mmHg})$ at the set rotational speed. The resistance 
clamp was then tightened to lower the pump flow rate at specified increments $(0.15,0.20$, or $0.25 \mathrm{~L} / \mathrm{min}$, depending on rotational speed setting). At each incremental pump flow rate, all parameters were recorded. The resistance clamp was also tightened to fully occlude the system, resulting in a pump flow rate of 0 $\mathrm{L} / \mathrm{min}$ and providing the maximum pressure of the pump at the set rotational speed. The system was reset with the resistance clamp completely opened, and this process was repeated for increasing rotational speeds (500 to 6000 RPM at 500 RPM increments). Experimental data for all test conditions were collected at a sampling rate of $400 \mathrm{~Hz}$ using PowerLab 16/35 (ADInstruments, Sydney, Australia) and recorded by LabChart 8 (ADInstruments, Sydney, Australia) data acquisition system software.

The total torque ( $\left.\boldsymbol{\tau}_{\text {Total }}\right)$ of the Inspired Pediatric VAD prototype at each speed and flow setting was calculated using the measured motor current $(\mathrm{I})$ and the derived torque constant $\left(\mathrm{K}_{\mathrm{t}}\right)$. The hydraulic torque $\left(\boldsymbol{\tau}_{\text {Hydro }}\right)$ of the impeller was based on the net torque between the calculated total torque $\left(\boldsymbol{\tau}_{\text {Total }}\right)$ of the fluid filled system, the total base torque of the DC motor $\left(\tau_{D C}\right)$ calculated with the derived linear equation, and the calculated average torque due to friction of the shaft and seal ( $\left.\boldsymbol{\tau}_{\text {Friction }}\right)$, (Equation 4.7).

$$
\tau_{\text {Hydro }}=\tau_{\text {Total }}-\tau_{D C}-\tau_{\text {Friction }}
$$




\subsubsection{Computational Fluid Dynamics (CFD) Model Simulations}

A series of CFD simulations were conducted to confirm the Inspired Pediatric VAD hydrodynamic performance and hydraulic torque values found empirically using the SFL model. A three-dimensional geometry (impeller and pump casing) of the VAD was generated using commercial computer aided design (CAD) software (SolidWorks 2019, Dassault Systèmes, Waltham, MA). The inlet and outlet of the pump casing geometry were capped (virtually sealed off) to establish the computational fluid volume, which included pump inlet and outlet ports, pump volute, and all surfaces of the impeller. The pump geometry was transferred into SolidWorks Flow Simulation, a parametric flow simulation tool (FloEFD, Siemens, Munich, Germany) that applies the finite volume method [61] to solve the three-dimensional Navier-Stokes equations [62], and meshed using the structured Cartesian immersed-body method. A rotating region was defined surrounding the pump impeller and the sliding-mesh method was used [63]. Blood was defined as a Newtonian fluid with a density of $\rho=1060 \mathrm{~kg} / \mathrm{m}^{3}$ at $37^{\circ} \mathrm{C}$ and a viscosity of $\mu=0.0035 \mathrm{~Pa}-\mathrm{s}$ [64], [66], [67]. All cases were run as time-dependent, transient simulations. Average inlet and outlet pressures and velocity as well as average torque (in the rotating axis) of the impeller and $\mathrm{X}, \mathrm{Y}$, and $Z$ translational forces (summation of forces acting on all impeller body surfaces) were used as convergence goals, with a convergence metric of $2 \%$ acceptance criteria. All simulations used a variable time-step to a physical flow time of 0.50 seconds or until all goals were sufficiently converged. 
The initial CFD simulation (Setup 1) (Figure 4-4A, B) for the Inspired Pediatric VAD included a mesh size of 317,965 cells with boundary conditions: flow velocity with fully developed parabolic profile at pump inlet, static pressure at pump outlet (100 $\mathrm{mmHg}$ ), and a real-wall stator condition (no-slip) for all pump casing walls. Based on deviation of initial results from CFD Setup 1 compared to

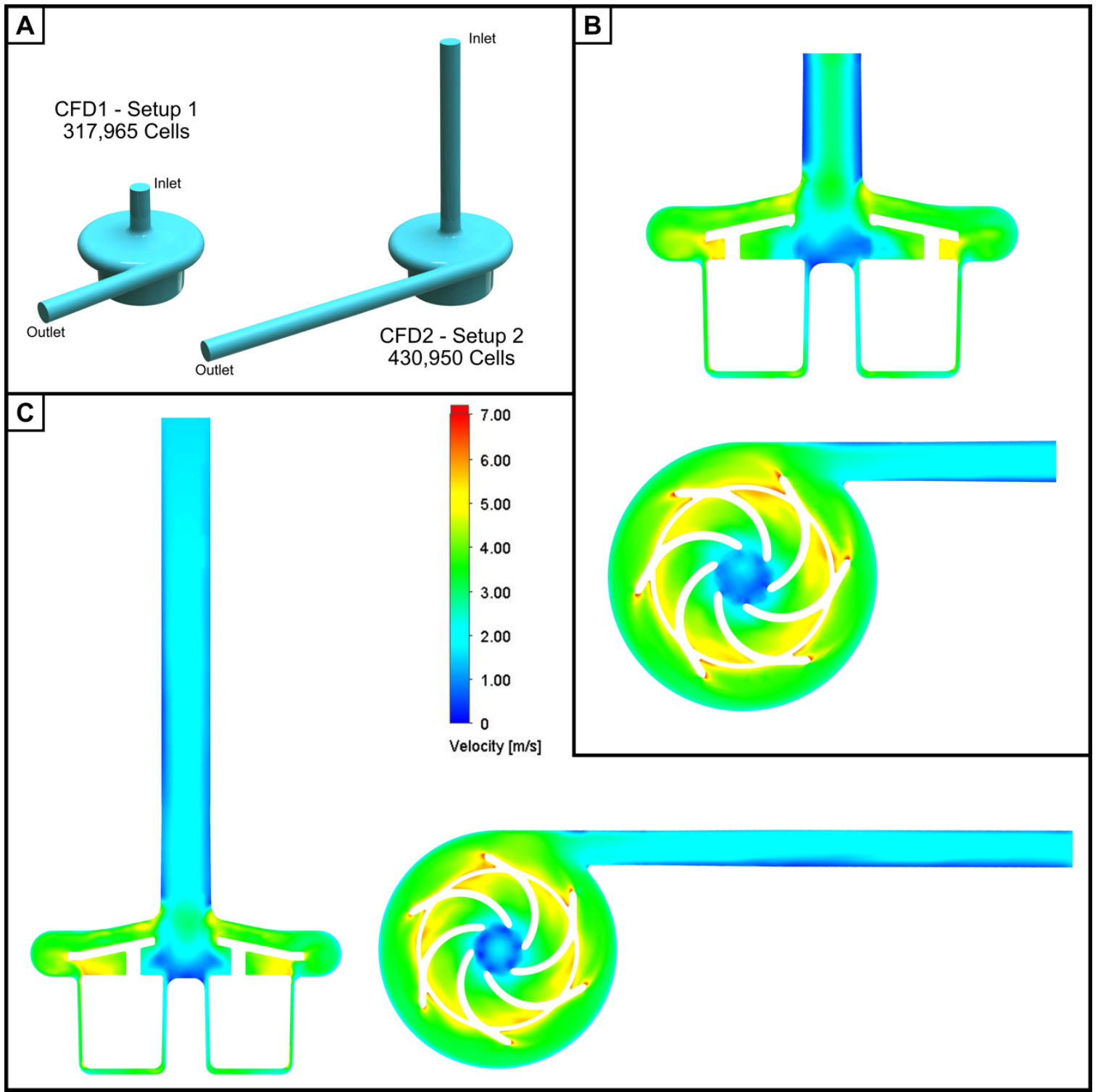

Figure 4-4. (A) Computational fluid volumes for CFD simulation Setup 1 and 2, (B) CFD Setup 1, Front and Top plane cut-plots of flow velocity for 5000 RPM at $3.00 \mathrm{~L} / \mathrm{min}$, (C) CFD Setup 2, Front and Top plane cutplots of flow velocity for $5000 \mathrm{RPM}$ at $3.00 \mathrm{~L} / \mathrm{min}$. 
empirical results from the SFL, an additional CFD simulation (Setup 2) was constructed to try to improve CFD predictions. CFD Setup 2 extended the inlet and outlet ports of the pump by $50 \mathrm{~mm}$ each and included a mesh size of 430,950 cells with boundary conditions: flow velocity with fully developed parabolic profile at pump outlet, environmental pressure at pump inlet (100 $\mathrm{mmHg}$ initial pressure), and a real-wall stator condition (no-slip) for all pump casing walls (Figure 4-4A, C). The flow velocity boundary condition was moved to the pump outlet in Setup 2 in an attempt to allow the flow at the pump inlet to fully develop naturally and not impart any assumed flow velocity field on the inlet of the impeller. Simulations using both Setups 1 and 2 were conducted at each test condition to produce pump pressure $(\Delta \mathrm{P})$, impeller torque, and impeller forces $(\mathrm{X}, \mathrm{Y}, \mathrm{Z}$ vectors) for each rotational speed and flow rate. An overview of SFL and CFD model experiments are presented in Figure 4-5.

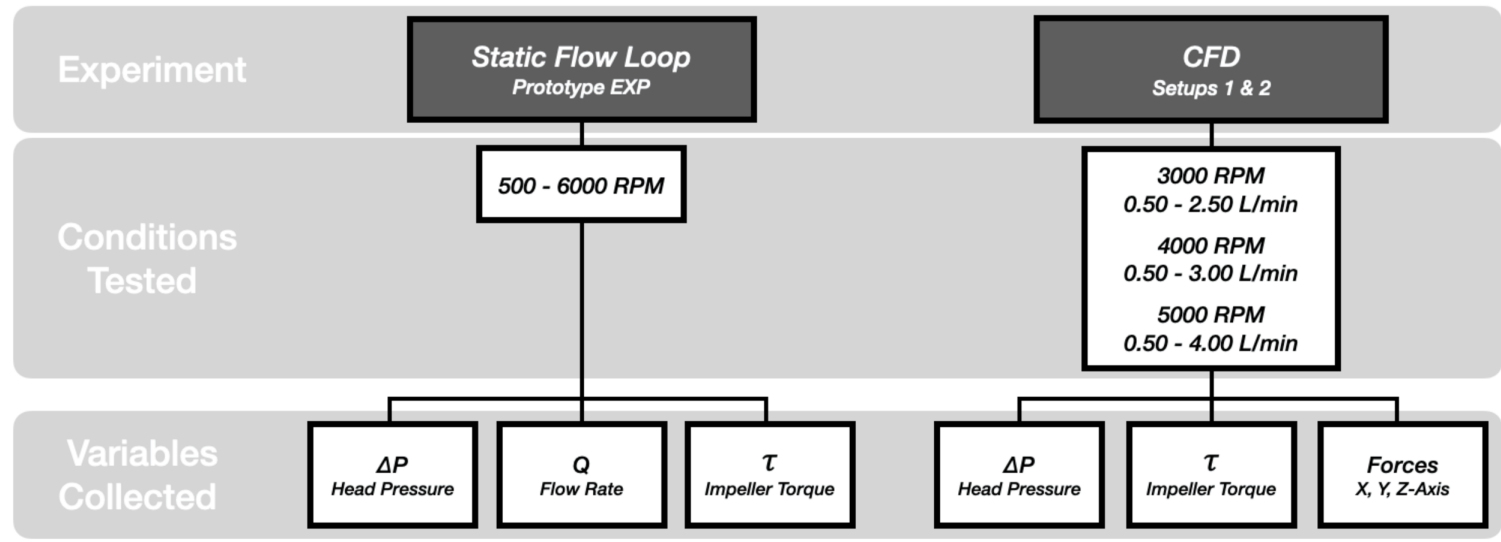

Figure 4-5. A flow chart detailing the SFL (using the empirical pump prototype, EXP) and CFD (using Setup 1 and 2) experiments, the tested pump conditions (rotational speed or flow rate and rotational speed) for each experiment, and the collected variables for each experiment (pump pressure, flow rate, impeller torque and impeller $x, y$, and $z$ forces). 


\subsection{Results}

\subsubsection{Empirical Pressure-Flow Curves}

With the flow rates $(\mathrm{Q})$ and pressures $(\Delta \mathrm{P})$ from the SFL model, the full hydrodynamic performance of the Inspired Pediatric VAD was observed for the first time over the operational range (500 - 6000 RPM) of the pump. These results were plotted to form characteristic pressure-flow curves (HQ curves) for the pump (Figure 4-6). The Inspired Pediatric VAD (prototype) was able to achieve flow rates as high as $4.27 \mathrm{~L} / \mathrm{min}$ with pressures as high as $422 \mathrm{mmHg}$ at 6000 RPM.

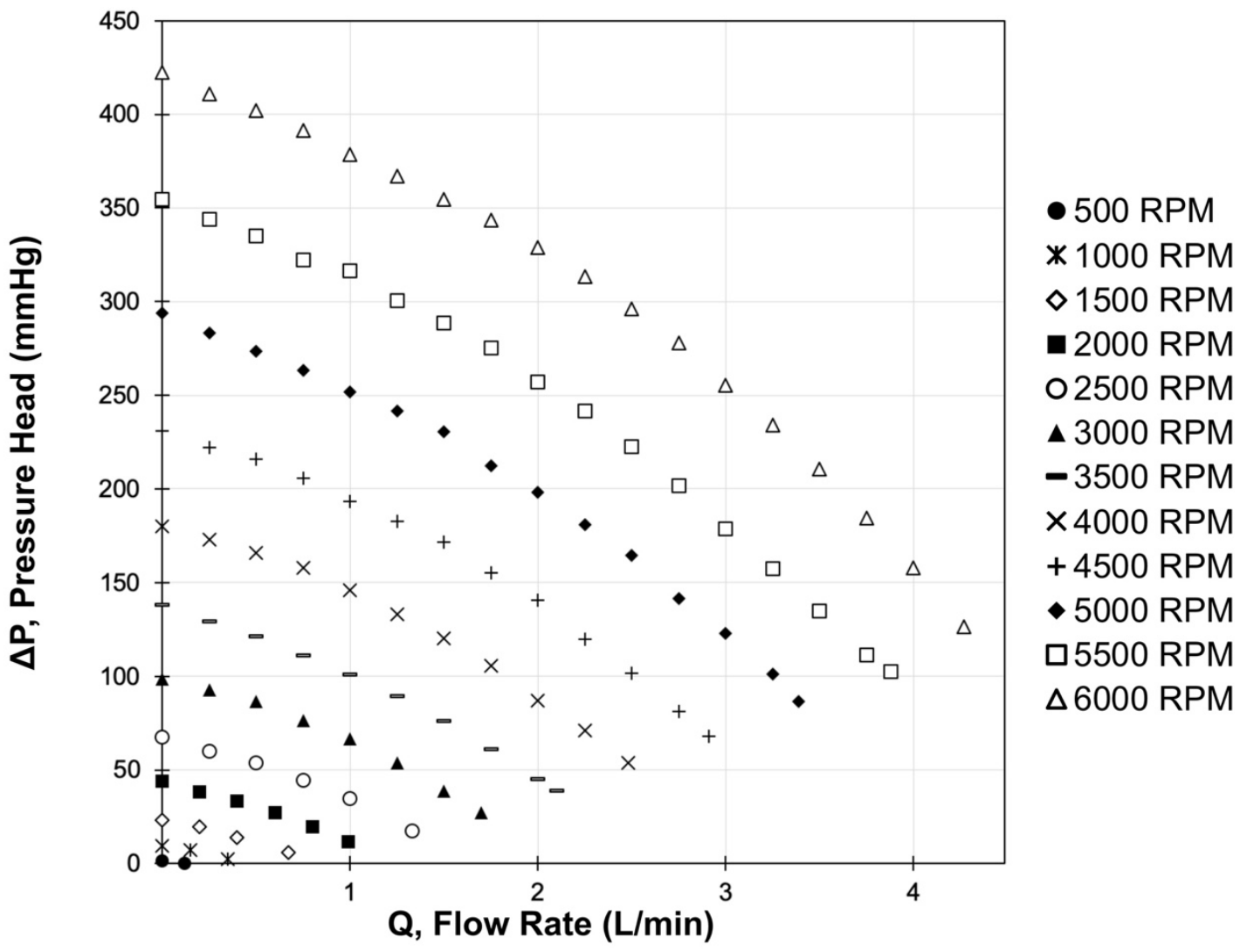

Figure 4-6. Characteristic HQ curves (pressure versus flow rate) for the Inspired Pediatric VAD pump empirical prototype for rotational speeds 500 to 6000 RPM. 


\subsubsection{Empirical Torque Curves}

The calculated hydraulic torques acting on the VAD impeller were plotted versus the pump flow rates producing characteristic torque curves for the pump (Figure 4-7). Over the operational flow range of the pump $(0.5-3.5 \mathrm{~L} / \mathrm{min})$, required torques to propel the fluid in the range of 0.97 to $15.56 \mathrm{mNm}$ were observed. Torques at the lower rotational range of the pump (500 - 2000 RPM) were low $(<1 \mathrm{mNm})$, with occasional negative values calculated due to the tare calculation (subtracting average friction and DC motor torque) to determine the hydraulic torque.

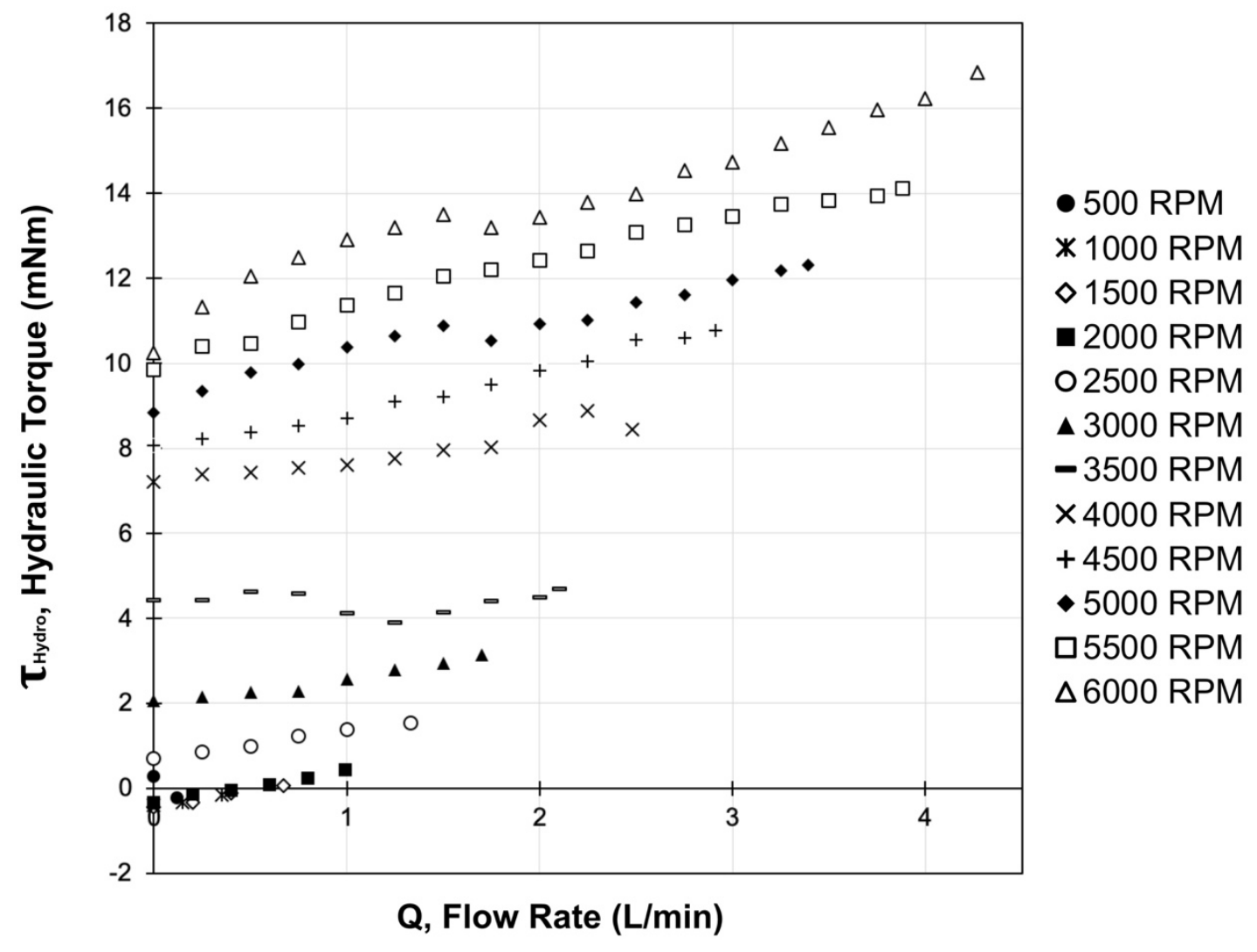

Figure 4-7. Hydraulic torque of the Inspired Pediatric VAD empirical prototype impeller/rotor versus flow rate for rotational speeds 500 to 6000 RPM. 


\subsubsection{Empirical vs. CFD Results}

Results from the prototype pump in the SFL compared with corresponding CFD simulations for Setups 1 and 2 are presented in Tables 4-1, 2, and 3. Simulation results for CFD Setups 1 and 2 varied, particularly at higher flow rates with differences as high as $19 \%$ for generated $\Delta \mathrm{P}$ values, while generated $\boldsymbol{\tau}_{\text {Hydro }}$ values were similar, with most values within $5 \%$ of one another for each simulation case. CFD Setup 2 typically produced lower overall $\triangle P$ values and higher force values than Setup 1. The difference between $\Delta \mathrm{P}$ values from Setup 1 to Setup 2 for CFD simulations produced varied results compared to the empirical prototype (EXP) for both $\Delta \mathrm{P}$ and $\tau_{\text {Hydro }}$ (Figures 4-8 and 4-9). The slope of the HQ curves generated with CFD are flatter compared to the more rounded curves from the empirical prototype. The slope of the torque curves generated with CFD are linear as are the curves from the empirical prototype, increasing with increased flow rate, though CFD values differ from empirical values for all rotational speeds. 
Table 4-1. Empirical results for the Inspired Pediatric VAD prototype in the SFL including generated pressure head $(\triangle P)$ and impeller/rotor hydraulic torque ( $\left.\boldsymbol{\tau}_{\text {Hydro }}\right)$ for selected rotational speed and flow cases (3000, 4000 , and 5000 RPM). $U=$ Unachievable, meaning the flow rate was not achievable at the given rotational speed by the prototype pump but was achievable in the CFD simulations.

\begin{tabular}{|c|c|c|c|c|c|c|c|c|}
\hline \multicolumn{9}{|c|}{ EXP - Empirical Prototype in Static Flow Loop } \\
\hline \multirow[b]{2}{*}{$\begin{array}{l}\text { Speed } \\
\text { (RPM) }\end{array}$} & \multirow[b]{2}{*}{$\begin{array}{c}\text { VAD Flow } \\
\text { (L/min) }\end{array}$} & \multirow[b]{2}{*}{$\begin{array}{c}\mathrm{P} 1 \\
(\mathrm{mmHg})\end{array}$} & \multirow[b]{2}{*}{$\begin{array}{c}\mathrm{P} 2 \\
(\mathrm{mmHg})\end{array}$} & \multirow[b]{2}{*}{$\begin{array}{c}\Delta \mathrm{P} \\
(\mathrm{mmHg}) \\
\end{array}$} & \multirow{2}{*}{$\begin{array}{c}\text { Hydraulic } \\
\text { Torque } \\
(\mathrm{mNm})\end{array}$} & \multicolumn{3}{|c|}{ Forces $(\mathrm{N})$} \\
\hline & & & & & & $x$ & $\mathrm{Y}$ & Z \\
\hline 3000 & 2.50 & $U$ & $U$ & $U$ & $u$ & - & - & - \\
\hline 3000 & 1.50 & 21.22 & -17.44 & 38.66 & 2.94 & - & - & - \\
\hline 3000 & 1.00 & 58.73 & -7.65 & 66.38 & 2.56 & - & - & - \\
\hline 3000 & 0.50 & 85.44 & -0.90 & 86.34 & 2.25 & - & - & - \\
\hline 4000 & 3.00 & $u$ & 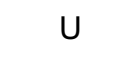 & U & U & - & - & - \\
\hline 4000 & 2.00 & 55.86 & -31.23 & 87.09 & 8.65 & - & - & - \\
\hline 4000 & 1.00 & 138.64 & -7.23 & 145.87 & 7.61 & - & - & - \\
\hline 4000 & 0.50 & 165.10 & -0.64 & 165.74 & 7.42 & - & - & - \\
\hline 5000 & 4.00 & U & U & $U$ & U & - & - & - \\
\hline 5000 & 3.00 & 57.67 & -65.33 & 123.00 & 11.96 & - & - & - \\
\hline 5000 & 2.00 & 168.92 & -29.36 & 198.28 & 10.92 & - & - & - \\
\hline 5000 & 1.00 & 244.80 & -6.91 & 251.71 & 10.38 & - & - & - \\
\hline 5000 & 0.50 & 273.43 & -0.20 & 273.63 & 9.78 & - & - & - \\
\hline
\end{tabular}


Table 4-2. CFD results for simulation Setup 1 including generated pressure head $(\Delta P)$ and impeller/rotor hydraulic torque ( $\boldsymbol{\tau}_{\text {Hydro }}$ ) for selected rotational speed and flow cases (3000, 4000, and 5000 RPM) and translational force values for the impeller/rotor in the $x, y$, and $z$ directions.

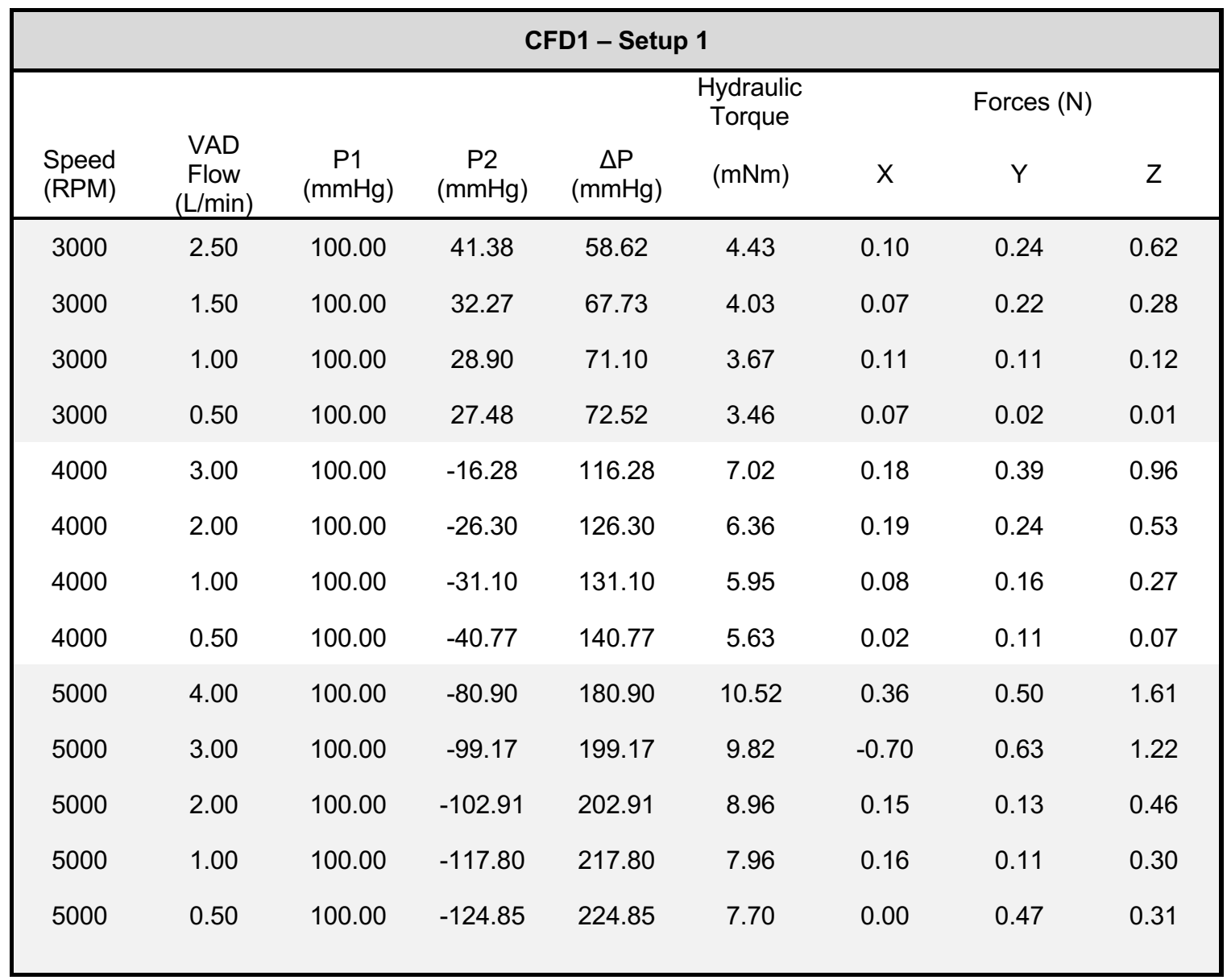


Table 4-3. CFD results for simulation Setup 2 including generated pressure head $(\Delta P)$ and impeller/rotor hydraulic torque $\left(\boldsymbol{\tau}_{\text {Hydro }}\right)$ for selected rotational speed and flow cases (3000, 4000, and 5000 RPM) and translational force values for the impeller/rotor in the $x, y$, and $z$ directions.

\begin{tabular}{|c|c|c|c|c|c|c|c|c|}
\hline \multicolumn{9}{|c|}{ CFD2 - Setup 2} \\
\hline \multirow[b]{2}{*}{$\begin{array}{l}\text { Speed } \\
\text { (RPM) }\end{array}$} & \multirow[b]{2}{*}{$\begin{array}{l}\text { VAD } \\
\text { Flow } \\
\text { (L/min) }\end{array}$} & \multirow[b]{2}{*}{$\begin{array}{c}\mathrm{P} 1 \\
(\mathrm{mmHg})\end{array}$} & \multirow[b]{2}{*}{$\begin{array}{c}\mathrm{P} 2 \\
(\mathrm{mmHg})\end{array}$} & \multirow[b]{2}{*}{$\begin{array}{c}\Delta \mathrm{P} \\
(\mathrm{mmHg})\end{array}$} & \multirow{2}{*}{$\begin{array}{c}\text { Hydraulic } \\
\text { Torque } \\
(\mathrm{mNm})\end{array}$} & \multicolumn{3}{|c|}{ Forces $(\mathrm{N})$} \\
\hline & & & & & & $x$ & $Y$ & Z \\
\hline 3000 & 2.50 & 140.45 & 93.11 & 47.34 & 4.46 & 0.12 & 0.28 & 0.78 \\
\hline 3000 & 1.50 & 159.85 & 97.52 & 62.33 & 3.91 & 0.08 & 0.24 & 0.52 \\
\hline 3000 & 1.00 & 166.26 & 98.90 & 67.36 & 3.76 & 0.05 & 0.20 & 0.39 \\
\hline 3000 & 0.50 & 172.03 & 99.72 & 72.31 & 3.38 & 0.05 & 0.11 & 0.24 \\
\hline 4000 & 3.00 & 189.61 & 90.06 & 99.55 & 6.90 & 0.25 & 0.35 & 1.22 \\
\hline 4000 & 2.00 & 211.24 & 95.59 & 115.65 & 6.30 & 0.13 & 0.29 & 0.84 \\
\hline 4000 & 1.00 & 229.17 & 98.90 & 130.27 & 5.85 & 0.14 & 0.23 & 0.52 \\
\hline 4000 & 0.50 & 232.73 & 99.72 & 133.01 & 5.30 & 0.12 & 0.14 & 0.49 \\
\hline 5000 & 4.00 & 235.96 & 82.36 & 153.60 & 10.49 & 0.39 & 0.56 & 1.85 \\
\hline 5000 & 3.00 & 272.88 & 90.09 & 182.79 & 9.51 & 0.26 & 0.42 & 1.39 \\
\hline 5000 & 2.00 & 293.48 & 95.60 & 197.88 & 8.89 & 0.24 & 0.25 & 1.01 \\
\hline 5000 & 1.00 & 308.18 & 98.89 & 209.29 & 8.02 & 0.16 & 0.20 & 0.80 \\
\hline 5000 & 0.50 & 318.43 & 99.72 & 218.71 & 7.24 & -0.10 & 0.01 & 1.02 \\
\hline
\end{tabular}




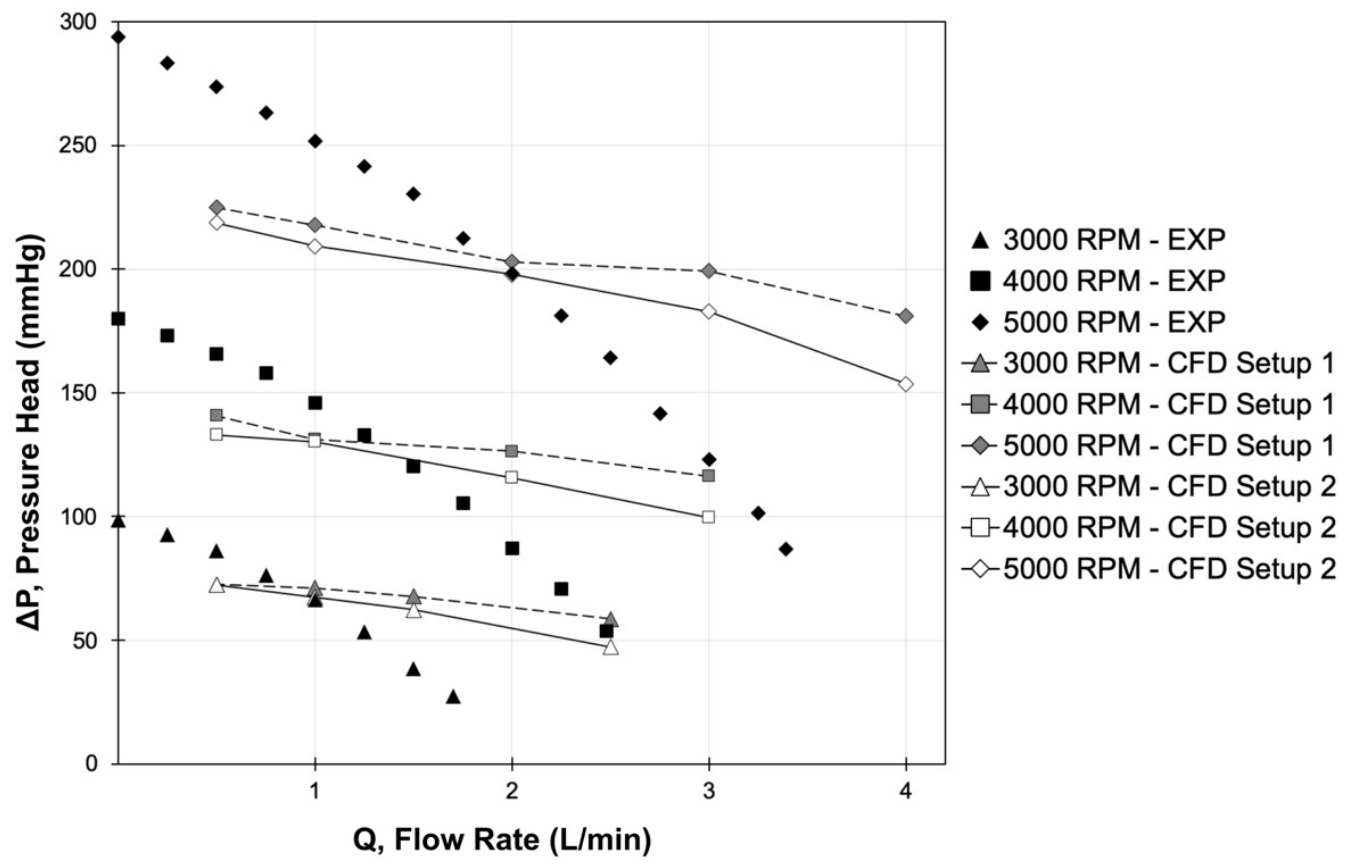

Figure 4-9. $H Q$ curves of the VAD empirical prototype versus generated curves from CFD simulations Setups 1 and 2 for rotational speeds 3000, 4000, and 5000 RPM.

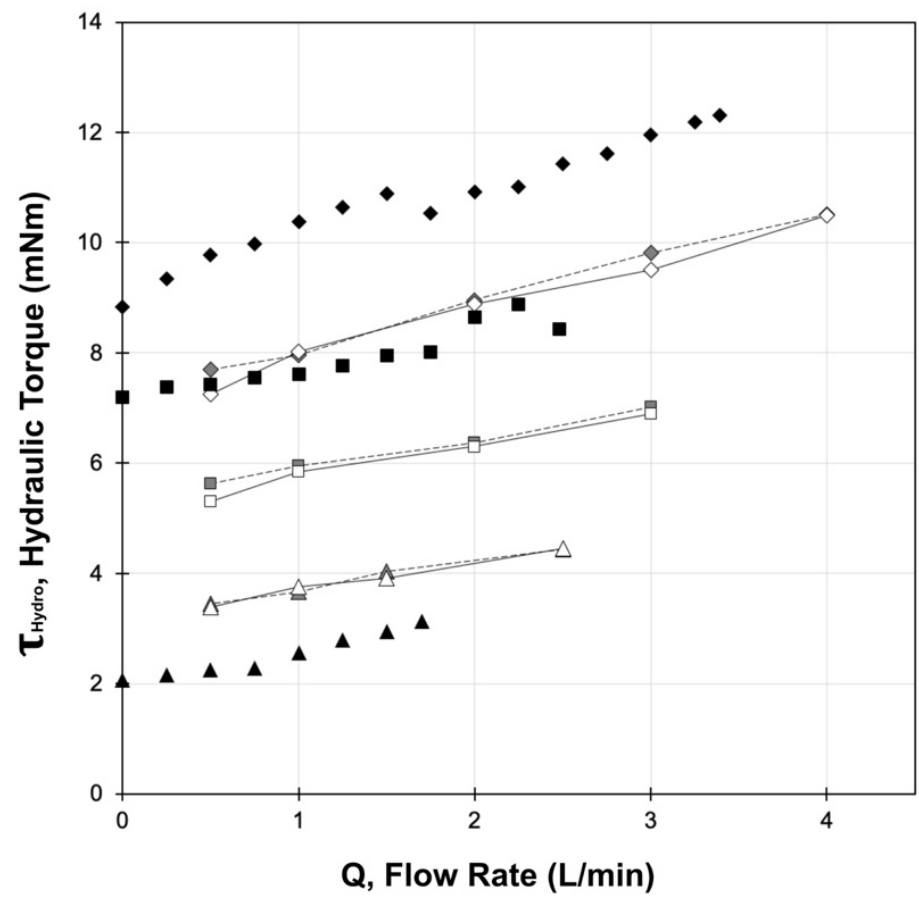

A 3000 RPM - EXP

4000 RPM - EXP

- 5000 RPM - EXP

$\triangle 3000$ RPM - CFD Setup 1

$\neg 4000$ RPM - CFD Setup 1

$\checkmark 5000$ RPM - CFD Setup 1

$\triangle-3000$ RPM - CFD Setup 2

$-\square-4000$ RPM - CFD Setup 2

$\checkmark 5000$ RPM - CFD Setup 2

Figure 4-8. Hydraulic torque of the VAD empirical prototype versus generated curves from CFD simulations Setups 1 and 2 for rotational speeds 3000, 4000, and 5000 RPM. 


\subsubsection{Impeller Forces}

The translational hydrodynamic force values for the impeller/rotor in the $\mathrm{x}$, $y$, and $z$ vectors that were generated from the CFD simulations are plotted in Figure 4-10. These values were derived as the sum of all force values acting on each body surface of the impeller/rotor in the CFD simulations. Tangential and radial forces ( $\mathrm{X}$ and $\mathrm{Y}$ vectors) were relatively small $(0.5 \mathrm{~N}$ or less) for both Setup 1 and Setup 2 for all rotational speeds. For both Setup 1 and 2, the axial force ( $Z$ vector) in the positive direction increased with increased rotational speed, likely due to the lift force created by the impeller rotation. Overall, Setup 2 produced greater force values in the $\mathrm{X}, \mathrm{Y}$, and $\mathrm{Z}$ directions than Setup 1, with typically increasing values as rotational speed and flow rate increased. In some cases, Setup 1 produced extremely varied values compared to Setup 2, particularly in the $X$ and $Y$ directions at 5000 RPM. 

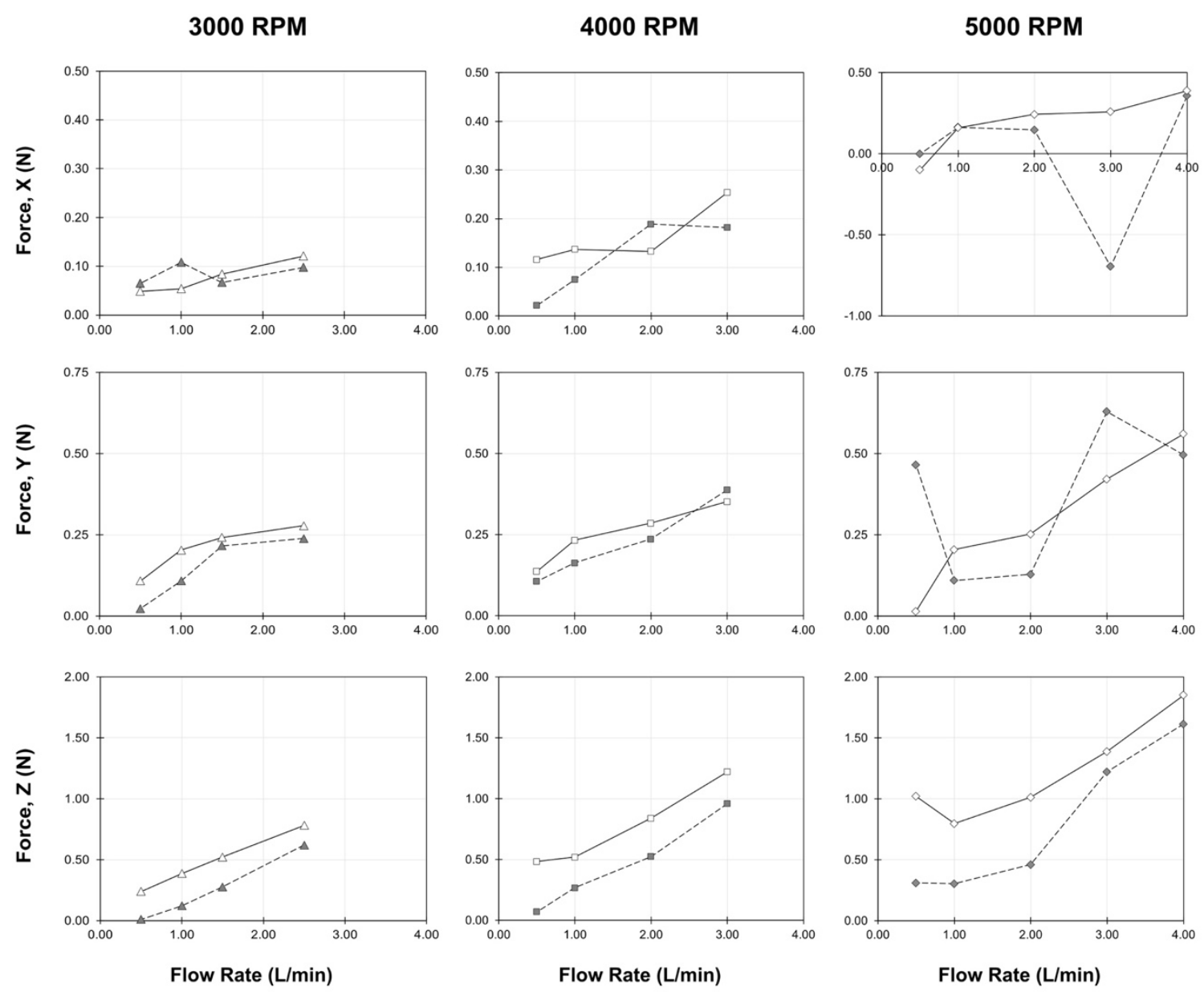

$\triangle 3000$ RPM - CFD Setup 1 - 4000 RPM - CFD Setup 1

$\leftrightarrow 5000$ RPM - CFD Setup 1

$\triangle 3000$ RPM - CFD Setup 2

$\square-4000$ RPM - CFD Setup 2

$\diamond 5000$ RPM - CFD Setup 2

Figure 4-10. Plots of translational $X, Y$, and Z-axis forces of the Inspired Pediatric VAD impeller/rotor generated from CFD Setups 1 and 2 at 3000, 4000, and 5000 RPM. 


\subsection{Discussion}

\subsubsection{Inspired Pediatric VAD Performance}

Hydrodynamic torque values for the impeller/rotor and the pump performance values (pressure and flow rate) for the Inspired Pediatric VAD were successfully quantified empirically using a prototype device in a SFL model over the pump operational range (500 - 6000 RPM). Additionally, HQ curves, hydrodynamic impeller/rotor torque, and translational force values in the tangential, radial, and axial directions for the impeller/rotor were successfully generated using CFD for select cases (3000, 4000, 5000 RPM). The shaft-driven prototype was able to achieve a flow rate as high as $4.27 \mathrm{~L} / \mathrm{min}$ against a pressure of $127 \mathrm{mmHg}$ at $6000 \mathrm{RPM}$. At the VAD's maximum intended design flow rate of $3.5 \mathrm{~L} / \mathrm{min}$, the pump was able to achieve pressures of $135 \mathrm{mmHg}$ and $210 \mathrm{mmHg}$ at 5500 and $6000 \mathrm{RPM}$, respectively.

These values differ from our prior CFD work [22], which predicted a flow of 3.5 L/min was achievable at $4255 \mathrm{RPM}$ at a pressure of $120 \mathrm{mmHg}$, as well as the CFD work done in this study (Setups 1 and 2) which predicted similar results. $\mathrm{HQ}$ curves generated via CFD Setups 1 and 2 exhibit a very flat slope, showing less reduction in $\Delta P$ with increased flow rate, $Q$, than the empirical prototype $H Q$ curves, which exhibit a more rounded slope with greater $\Delta P$ fall-off as $Q$ is increased. Though values between CFD and empirical hydraulic torque curves differed, both sets of curves followed the same trend of a linear increase in torque following an increase in flow rate. Attempts to improve the CFD model to match the empirical results with the implementation of Setup 2 were not 
successful even though the $\mathrm{HQ}$ and torque curve trends between the two CFD Setups were similar. The CFD simulations in this study were not as accurate in predictions compared with the empirical findings and the CFD model needs to be improved to more accurately reflect actual pump performance.

There are many potential factors that could contribute to the inaccuracy of the CFD predictions including modeling of the inlet and outlet tubing, potential differences in surface roughness and dimensions, and assumptions made with the CFD simulation model (see Study Limitations section). Additionally, the flow paths within the Inspired Pediatric VAD are very complex. In addition to the primary flow path through the impeller and out the pump outlet, there is a secondary flow path of fluid circulating around the outer perimeter of the rotor body and through the center opening, and a tertiary path of fluid recirculating between the top of the impeller shroud and upper pump housing. These flow paths were designed to provide washing of all pump surfaces to negate thrombus formation but are also detrimental to the overall hydraulic efficiency of the pump as not all fluid adheres to the primary impeller path, and this is common in many RBP designs. Although these flow paths are seen in the present CFD results the simulation may not be accurately modeling these flow "leakages", causing the pump to perform better in simulations than in the actual prototype experiment [40]. CFD analysis of these specific flow path regions will be conducted in future studies. 


\subsubsection{Indications for MagLev Motor Design}

Empirical $\mathrm{HQ}$ and torque findings from this study provide insight into the design requirements for the MagLev motor system to be developed. For the spinning motor component, the MagLev system will need to be able to spin the impeller/rotor between 2000 and 5500 RPM and produce torques between 0.10 and $14 \mathrm{mNm}$ to achieve the desired pump operational range from 0.5 to 3.5 $\mathrm{L} / \mathrm{min}$ at 40 to $150 \mathrm{mmHg}$. Although translational force values were not able to be produced empirically in this study, and the CFD predictions differed from empirical findings, CFD predictions from this study will be used as an estimate for impeller/rotor forces until better predictions are achieved. Overall, the generated force values indicate that with increased rotational speed and flow rate, the impeller/rotor will rise upward inside the pump housing (positive Z-axis) due to increasing axial force and the levitation component of the MagLev system will have to contend with levitating not only the mass of the impeller/rotor, but also counteracting lift forces as high as $1.9 \mathrm{~N}$ produced by the impeller.

\subsubsection{Study Limitations}

Results from this study highlight some of the limitations related to computational modeling, prototype fabrication methods, and mock loop models. The difference in CFD and empirical results were not entirely unexpected as CFD simulations tend to represent a best-case scenario for pump performance with many assumptions made that often do not translate to physical bench testing. There have been many studies that have examined blood pump designs that 
compare empirical and CFD results with varied levels of success toward achieving agreement between the two approaches [35], [40], [85]-[88]. Typically, CFD models must be customized for each pump geometry to more accurately match the conditions of the physical testing set-up [89]. Some assumptions in the CFD simulations made in this study included treating blood as a Newtonian fluid and assuming steady laminar flow, both of which were done to reduce simulation complexity and calculation time. Additionally, the pump geometry components in the CFD simulations were assumed to be perfectly smooth, with no surface variation, and the inlet and outlet ports on the pump were straight rigid tubes, which was not the case with SFL model testing as the pump inlet and outlet were connected to compliant tubing that curved in various areas. All of these assumptions may have contributed to differences observed between CFD model predictions and SFL model empirical findings. We plan to further investigate these challenges with continued CFD model development and comparison to in vitro model testing.

Another limitation of this study is the production of pump prototype components via additive manufacturing. Since the Inspired Pediatric VAD is in early development phase, additive manufacturing was utilized to produce prototype components to reduce cost and allow for rapid changes in component design. Unfortunately, the accuracy of the impeller blade geometry and pump housing volute produced by 3D printing may have varied from the solid model used in the CFD simulations due to printing tolerances [90]. The SLA printing tolerances using the WaterShed material were \pm 0.002 inches in the $X / Y$ 
dimension and \pm 0.005 inches in the $Z$ dimension, not accounting for material shrinkage. The Pediatric VAD has many small features with typical wall thicknesses around 0.020 inches, so accuracy is key to produce these dimensions. Additionally, SLA printed plastics are not perfectly smooth, creating surfaces with roughness between $2-4 \mu \mathrm{m}$ [91]. In future studies we will utilize high fidelity measuring techniques and systems to inspect pump components against CFD solid models.

Lastly, hydraulic torque of the impeller/rotor in the SFL experiment was not measured (i.e. with a dedicated torque transducer), but was calculated via the PMDC motor, which may affect the accuracy. Although these methods each have limitations, CFD and bench-top models represent two practical techniques that can be used to efficiently design blood pumps and together provide useful information to make informed design decisions.

Despite these limitations, this research resulted in data that suggests the Inspired Pediatric VAD will be able to generate the desired pressures and flow rates to provide adequate circulatory support for pediatric heart failure patients. These empirical performance results obtained in this study compare favorably to other blood pumps used in the pediatric population including the BP-50, RotaFlow, Deltastream DP3, and Thoratec PediMag [18], [49], [51], [53]. A comparison of the torque and force data generated by this study to other blood pumps is difficult since these values are directly determined by the pump's impeller/rotor diameter and size as well as hydrodynamic conditions. The torque 
and force data generated in this study will be instrumental in designing the specific MagLev motor for the Inspired Pediatric VAD.

\subsubsection{Further Thoughts on CFD Predictions and Future Work}

Initial computational modeling (CFD) and proof-of-concept bench testing was conducted to characterize the hydrodynamic performance of the Inspired Pediatric VAD. As anticipated, variances between CFD model predictions and experimental test conditions were identified. In addition to the limitations detailed in the previous section, other key aspects of the CFD simulations will be considered to help identify design weaknesses and engineering challenges, which will be a focus of improvement with future development work.

Accurate and sufficient discretization (meshing) of the Pediatric VAD model is critical to achieving accurate numerical simulation predictions. In using the Sliding Mesh Method [63] in SolidWorks Flow Simulation, there are multiple ways to define the three-dimensional rotating region that encompasses the impeller/rotor body that may affect the meshing at this critical boundary. The immersed body structured meshing method used in SolidWorks Flow Simulation initiates the meshing process by creating a global mesh of the entire simulation geometry. This mesh is then refined (cells are broken into finer cells for increased resolution) by the user to identify key areas of interest, where the geometry is complex, or where complicated flow conditions (i.e. vortices, turbulence, etc.) are predicted to occur. In addition to refining the global mesh, local meshes of key geometries, surfaces, or fluid regions may also be created. 
Two local meshes were defined in this study, one surrounding all impeller/rotor surfaces, and a second specifically at the pump housing walls in the radial blood gap between rotor and housing. These two local meshes had increased refinement in addition to the refinement of the global mesh to ensure that the discretization of the fluid volume at the critical area between the rotating region and stationary pump wall was sufficient (adequate resolution). Further, a mesh independence analysis was conducted for the Inspired Pediatric VAD V2 using four different levels of local and global mesh refinement at the $Q=3.5$ $\mathrm{L} / \mathrm{min}$ and $\mathrm{n}=4255 \mathrm{RPM}$ test condition. Convergence goals $\Delta \mathrm{P}$ (generated pressure) and impeller torque were used to evaluate the strength of each meshing scheme (Appendix B, Table B-2 and Figures B-1, B-2). Meshing Scheme 3 showed less than a $1 \%$ difference in converged $\Delta P$ value and less than a $3 \%$ difference in converged torque value compared to the much finer meshing with Scheme 4. Therefore, meshing Scheme 3 was used for CFD Setup 1 (317,965 cells) and CFD Setup 2 (430,950 cells); however, continued assessment of mesh refinement is warranted given the observed variances between predicted and empirical findings. In future work, different geometries will be used to define the rotational region surrounding the impeller/rotor resulting in variances to the mesh and mesh refinement at the critical radial gap. Additional simulations will be performed in a parametric fashion to observe the effect of different geometry definition and meshing techniques at this critical area.

An additional CFD simulation (Setup 2) was conducted to observe the effect of the length of the pump inlet and outlet ports on the measured average 
static pressure at each of the inlet/outlet boundaries. In CFD Setup 1 (shorter inlet/outlet ports), a parabolic velocity profile was defined at the inlet boundary to simulate fully developed flow into the pump. In CFD Setup 2, a parabolic profile was not initially defined, and during the simulation, a parabolic velocity profile was created "naturally" due to the extended length of the inlet tube. The working hypothesis was that the "artificial" velocity profile used in Setup 1 may have led to incorrect numerical predictions at the simulation boundaries. After completing multiple simulations with both setups, the inlet/outlet length and parabolic velocity profile were found to have little effect on the predicted outcome (less than 10\% difference for most predictions). This finding suggests that meshing appears to be a critical aspect in attaining accurate predictions in SolidWorks Flow Simulation. Therefore, the CFD model will continue to be modified to improve the meshing of the Pediatric VAD model in future studies.

Laminar flow was assumed in this study to reduce the computational load of each simulation and in the calculation of the predicted flow conditions within the Pediatric VAD. Reynolds numbers $(R e)$ were calculated for the pump inlet and impeller regions of the VAD geometry following methods suggested by Fraser et al. [68], [69] for predicting flow conditions for centrifugal blood pumps (Table B-1 and Table E-1). The decision to model strictly laminar flow was made based on the Reynolds number predictions and also after reviewing many similar studies where laminar flow was modeled for pediatric centrifugal pumps [53], [67]. Based upon the results of VAD V2 simulation and bench testing, the Inspired Pediatric VAD was re-designed with a new VAD geometry (VAD V3). 
CFD simulations of VAD V3 design were conducted using laminar and turbulent solvers in SolidWorks Flow Simulation to compare results between the two methods (Appendix F). For these simulations, blood was assumed to be a Newtonian fluid. The two solvers produced varied results for predicted $\Delta \mathrm{P}$ and impeller torque values $(5-15 \%$ difference for all predicted values, Table F-1, Figure F-1). However, the slopes of predicted HQ curves using the two methods were almost identical (slope of -9.1 for Laminar solver vs. -11.3 for Turbulent solver), suggesting that there is merely an offset in predicted values between the two methods rather than a disagreement between the overall CFD simulations (average offset of $20.6 \pm 3.6 \mathrm{mmHg}$ ). In future work, the effects of turbulent flow will be investigated, which may be better modeled using a more robust turbulent flow solver (ANSYS Fluent).

In SolidWorks Flow Simulation, convergence of the simulation solution is determined by assessing integrated quantities from the solution, such as average flow rate and average static/total pressure, for which these user-defined values are selected as "Goals". In other CFD software, convergence is often measured by observing changes in the residuals of the Navier-Stokes conservation of momentum and energy equations. Goal quantities in SolidWorks tend to function in a similar fashion to solution residuals, and criteria are generally set to determine convergence based on changes in the Goal quantities (e.g. when the solution is converged, Goal quantities should have limited changes, if any, to their values). 
Goal quantities of flow rate, velocity, and average static pressure at both the inlet and outlet boundaries were used in this study, with strict convergence criteria (less than a $2 \%$ change in Goal value). For all simulations, the Goal quantities typically converged within the first 0.10 to 0.15 seconds of physical flow time, which was observed in the curve plots of the Goal values vs. Time and the Goal convergence criteria (changes of less than $2 \%$ in the Goal solution value between iterations). All simulations were run to a physical flow time of 0.5 seconds. In addition to Goal convergence criteria, adequate time step values were used for each simulation (detailed in Appendix B.1). In future work, the effect of time step and Goal convergence criteria on the accuracy of the CFD numerical predictions will be used as an additional metric for comparison to the prototype experimental findings will be investigated.

\subsection{Conclusion}

While our previously reported work focused on the initial design of the Inspired Pediatric VAD, this article focused on the design of the novel MagLev motor for the Inspired Universal system. The data acquired in this investigation will be instrumental in advancing the design of the MagLev motor to ensure that it is able to produce adequate torque to drive the impeller/rotor, translational forces to stabilize the impeller/rotor, and responsiveness to accurately position the impeller/rotor within the pump housing at all times. Future work will include the design, fabrication, and testing of the MagLev motor and controller for the Inspired Universal MagLev system with pre-clinical testing in mock flow loop and 
large animal models. The concept of the Inspired System as a universal platform for multiple pumps is extremely innovative and coupled with the fully magnetically levitated impeller/rotor design of the Pediatric VAD (and all future VADs), this system aims to make a lasting impact. 


\section{CHAPTER 5 : DEVELOPMENT OF INSPIRED THERAPEUTICS PEDIATRIC VAD: COMPUTATIONAL ANALYSIS AND CHARACTERIZATION OF VAD V3}

\subsection{Introduction}

In the United States, 14,000 children are hospitalized with heart failure (HF) and HF-related conditions each year, with an overall mortality rate of $7 \%$ [10]. The population of pediatric patients listed for heart transplantation (HT) continues to grow with approximately 600 new listings each year while the population of donor organs remains static [92], [93]. With limited treatment options, mechanical circulatory support (MCS) has emerged as an important modality for improving survival and maintaining growth and development in pediatric patients [9]. Unfortunately, the development of suitable MCS devices for

Authors: ${ }^{1}$ Landon H. Tompkins, MEng., ${ }^{2}$ Barry N. Gellman M.S., ${ }^{3}$ Gino F. Morello, ${ }^{4}$ Steven R. Prina Ph.D., ${ }^{1}$ Thomas Roussel Ph.D., ${ }^{1}$ Jonathan A. Kopechek Ph.D., ${ }^{5}$ Stuart J. Williams Ph.D., ${ }^{2}$ Priscilla C. Petit, ${ }^{6}$ Mark S. Slaughter M.D., ${ }^{1,6}$ Steven C. Koenig Ph.D., ${ }^{1,2,6}$ Kurt A. Dasse Ph.D.

Department of Bioengineering, University of Louisville, Louisville, $K Y$

Inspired Therapeutics LLC, Merritt Island, FL

Veritium Research LLC, Fort Lee, NJ

BLDC Designs LLC, Rocklin, CA

Department of Mechanical Engineering, University of Louisville, Louisville, $K Y$

6 Department of Cardiovascular and Thoracic Surgery, University of Louisville, Louisville, KY 
pediatric patients has fallen far behind the efforts for the adult population. To address this clinical need, Inspired Therapeutics (Merritt Island, FL) and the University of Louisville (Louisville, KY) have been developing the Inspired Pediatric VAD, a new single-use rotary blood pump for use with the Inspired Universal MagLev System that is being designed to provide up to 30-days of circulatory support for pediatric heart failure patients (Figure 5-1).

The unique concept of the Inspired System is to enable clinicians to treat many different indications for use (cardiopulmonary bypass, HF, respiratory

\section{INSPIRED PEDIATRIC VAD V3}

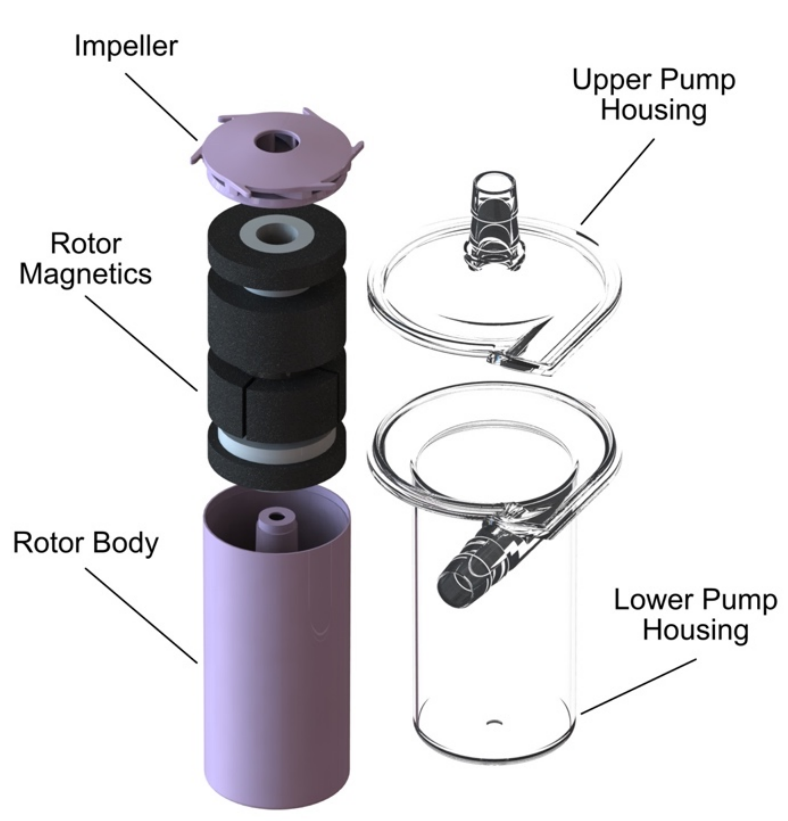

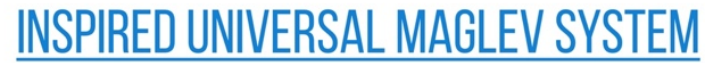

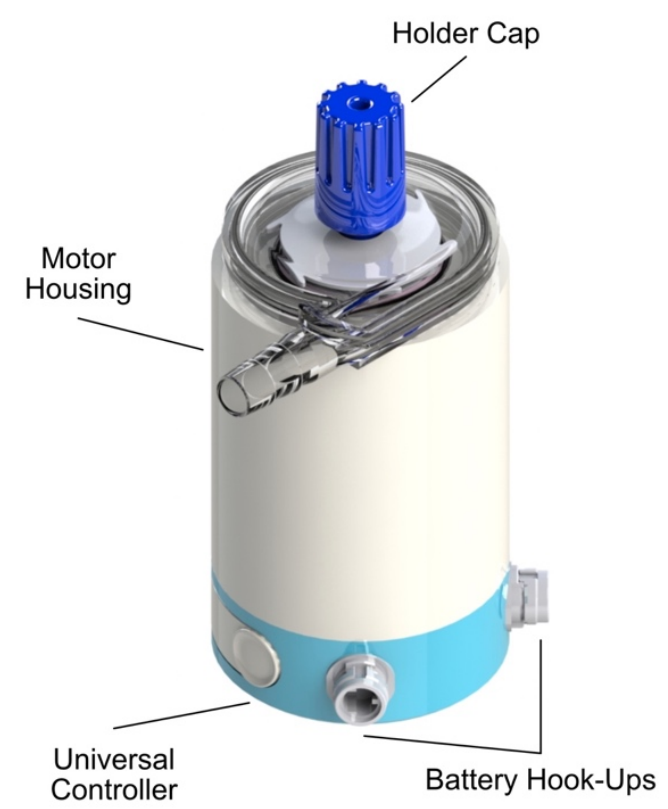

Figure 5-1. Exploded view of the Inspired Pediatric VAD V3 (left) identifying the rotating body (impeller, rotor body, and rotor magnetics) and the stationary body (upper and lower pump housing), and conceptual rendering of the complete Inspired Universal MagLev System with attached Inspired Pediatric VAD (right) identifying the motor housing, universal controller module with battery hook-ups, and the VAD inlet holder cap, which holds the impeller/rotor in a stationary position for transport. 
assist) and patient populations (neonate, pediatric, adult) using one common, compact, universal motor/controller to power and drive an array of modular disposable pumps. The initial development for this system focused on the pediatric pump (Inspired Pediatric VAD), for which the design, computational fluid dynamics (CFD) analysis, and in vitro testing of the impeller and pump housing have been previously described [22]. The resulting data were applied in the development of a novel magnetically levitated (MagLev) motor system that freely levitates and rotates the pump impeller/rotor. Subsequently, the prototype Inspired Pediatric VAD was redesigned to accommodate the MagLev system components. In this article, we describe the computational characterization of the newest iteration of the Inspired Pediatric pump (VAD V3) and detail its predicted hydrodynamic performance compared to earlier prototypes (VAD V1, VAD V2). Additionally, we detail the complicated velocity flow fields present in the new design and the predicted hemocompatibility through shear stress and residence time analysis.

\subsection{Methods}

\subsubsection{Inspired Pediatric VAD V3 Design}

The initial design of the Inspired Pediatric VAD impeller, rotor body and pump housing were created before the development of the MagLev motor system. The prototypes (V1, V2) were initially designed with a single magnetic component inside the rotor body below the impeller (Figure 5-2, Inspired Pediatric VAD V2). The VAD impeller/rotor hydrodynamic torques and forces for these prototypes were characterized and used to develop the MagLev motor. Specifically, the MagLev motor design required lengthening the lower pump 
housing and rotor body to accommodate the rotor magnetics. Additionally, due to molding requirements, wall thicknesses of the lower pump housing and rotor body needed to be adjusted to maintain the gap between rotor and stator components and the blood gap between impeller/rotor and housing. A center post in the lower pump housing, which the impeller/rotor rotated around in VAD V2, was also removed due to molding requirements, leaving a center opening in

Inspired Pediatric VAD V2

Inspired Pediatric VAD V3
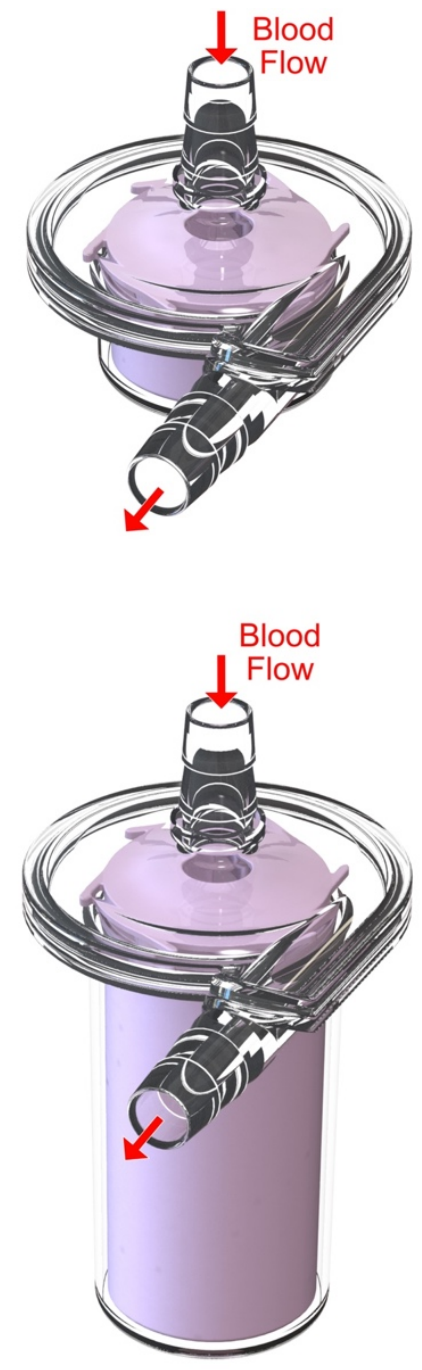
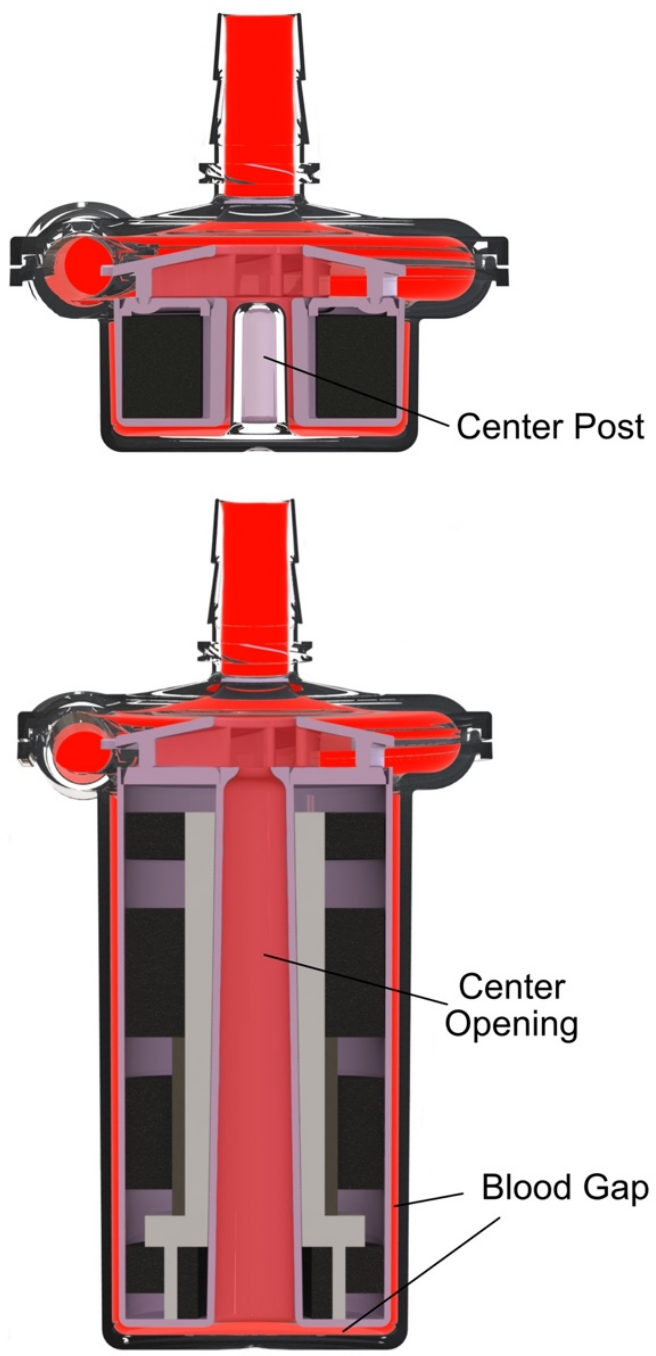

Figure 5-2. Comparison of the Inspired pediatric VAD V2 (top left) and V3 (bottom right) illustrating differences in rotor body and pump housing design, which resulted in an increase in priming volume from 9.1 to $14.5 \mathrm{ml}$, and removal of the center post (V3). 
the rotor body. The re-design (Figure 5-2) resulted in a unique rotary blood pump geometry with a long rotor body and an increase in priming volume from $9.1 \mathrm{ml}$ (V2) to $14.5 \mathrm{ml}(\mathrm{V} 3)$.

\subsubsection{MagLev Motor Design}

The MagLev motor development required creating a combined electric motor and magnetic levitation system that would allow the impeller/rotor of the VAD to remain suspended and rotating in the blood without contacting the pump housing walls over a range of speeds (500 - 6000 RPM), pressures (up to 150 $\mathrm{mmHg})$, and flows $(0.5-3.5 \mathrm{~L} / \mathrm{min})$. Passive static magnetics were selected for the radial bearing system with active levitation in the axial direction. Since complete static/passive stable magnetic levitation is not possible in all three dimensions ( $\mathrm{x}, \mathrm{y}, \mathrm{z}$-axis) [94], a voice coil magnetic actuator (VCA) was selected to actively control the axial position (levitation) of the floating/rotating assembly of components. The VCA is part of a position feedback system that senses the axial position of the floating impeller/rotor assembly using Hall effect sensors and varies the current in the VCA coil to produce an axial magnetic force that in turn controls the impeller/rotor position. Active axial positioning of the pump impeller/rotor is a novel aspect of the Inspired System, and the controller allows for precise positioning of the moving member and maintenance of axial blood gaps. Previous MagLev blood pumps such as the Thoratec CentriMag system use passive axial positioning with active radial positioning, which allows the impeller/rotor to rise and fall within the pump housing with changes in rotational 
speed, flow, and system pulsatility. Changes in the axial blood gap can cause the formation of vortices that are detrimental to pump efficiency and hemocompatibility [95], and in the case of the CentriMag, increasing the axial blood gap increases the fluid shear stress and residence time and the lift force generated by the rotating impeller [96]. A slotless brushless DC motor that met all

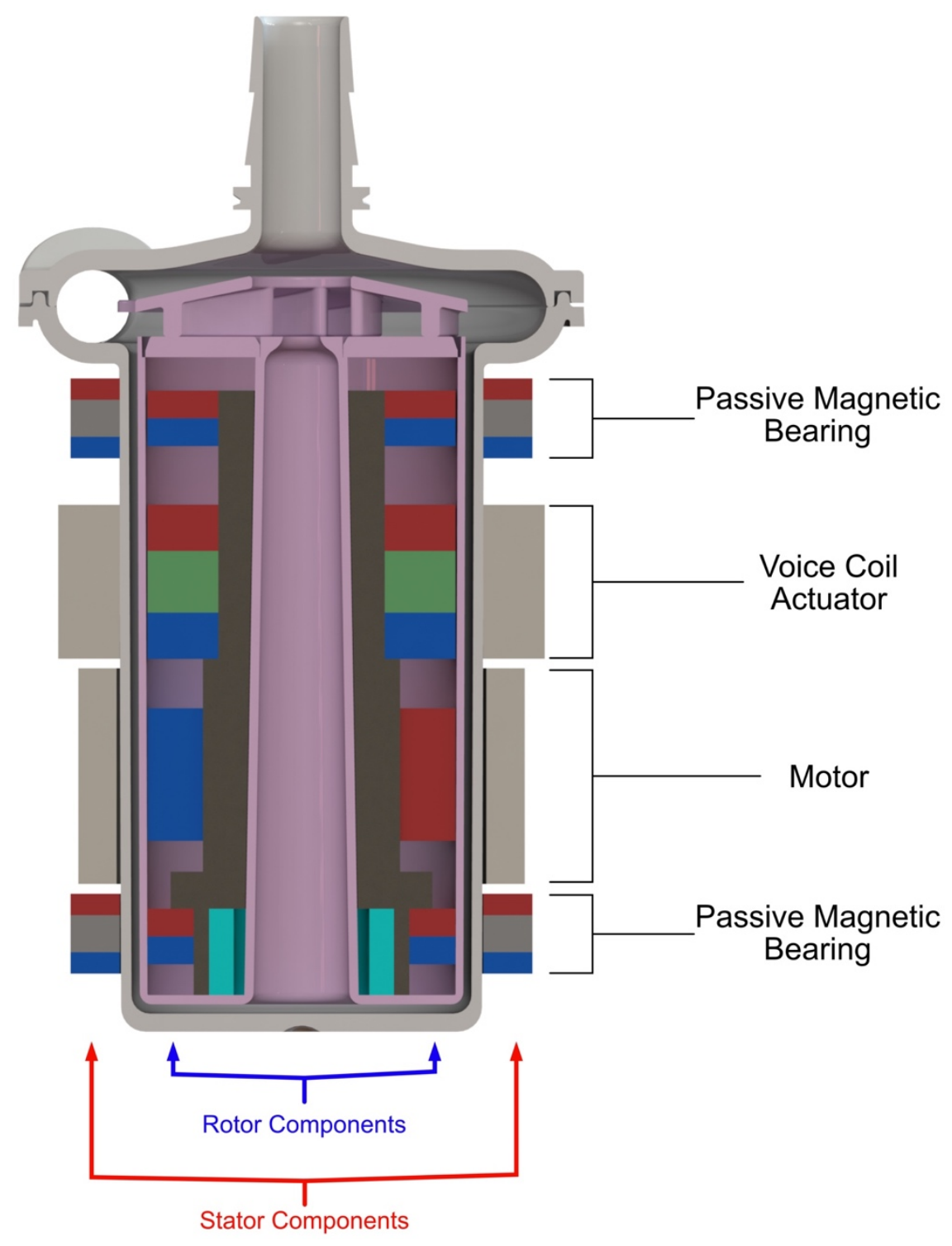

Figure 5-3. Cross-section view of the MagLev motor design for V3 showing rotor and stator components and identifying the passive magnetic bearings, voice coil actuator and brushless permanent magnet motor. 
of the torque-speed operating requirements was developed to rotate the actively levitated impeller/rotor. A passive magnetic bearing (PMB) system was designed to control the radial positioning of the impeller/rotor within the pump housing, maintaining the necessary blood gaps around the perimeter of the rotor. A crosssection of the MagLev motor design V3 is illustrated in Figure 5-3.

\subsubsection{Computational Fluid Dynamics (CFD) Simulation}

Computational fluid dynamics (CFD) analysis techniques were used to evaluate the hydrodynamic performance, impeller/rotor torques and forces, and hemocompatibility of the redesigned Inspired Pediatric VAD V3. Threedimensional geometry (impeller and pump casing) was generated using commercial computer aided design (CAD) software (SolidWorks 2019, Dassault Systèmes, Waltham, MA). The pump geometry was transferred into SolidWorks Flow Simulation, a parametric flow simulation tool (FloEFD, Siemens, Munich, Germany) that applies the finite volume method [61] to solve the threedimensional Navier-Stokes equations [62]. The computational fluid volume for the simulation was established, including the pump inlet and outlet ports, pump volute, and all surfaces of the impeller, and a rotating region was defined surrounding the pump impeller and the sliding-mesh method was used to simulate impeller rotation [63]. Boundary conditions for the simulation were set as: (1) fully developed parabolic profile flow velocity field at the pump inlet, (2) constant static pressure at the pump outlet, and (3) real-wall stator condition (noslip) for all pump housing walls. In previous analyses of the Inspired Pediatric 
VAD V2, blood was modeled as a Newtonian fluid for simplicity; however, SolidWorks Flow Simulation provides limited data output in terms of shear rates and forces in the flow domain when simulating this type of fluid. To address this limitation for analysis of the $\mathrm{V} 3$ design, blood was modeled as a Non-Newtonian fluid via the Power Law Model [97] with a density of $\rho=1060 \mathrm{~kg} / \mathrm{m}^{3}$ at $37^{\circ} \mathrm{C}$, a minimum viscosity of $\mu=0.003038 \mathrm{~Pa}-\mathrm{s}$, a consistency index of $0.01217 \mathrm{~Pa}-\mathrm{s}$, and a power-law index of 0.7991 [98].

To assess flow turbulence, Reynolds numbers $(\mathrm{Re})$ were calculated [68], [69] for the pump inlet port and the impeller to determine whether turbulent flow may occur (Appendix E, Table E-1). All calculated Reynolds numbers for the impeller at the simulated rotational speeds of 3000,4000 , and 5000 RPM were in the laminar flow range. The calculated Reynolds numbers for the pump inlet were in the laminar flow range for flow rates $0.5-2.0 \mathrm{~L} / \mathrm{min}$ and were in the laminar-toturbulent transition area $(2300<\operatorname{Re}<4000)[70]$ for flow rates between $2.5-4.0$ L/mins, as has been reported in the modeling of centrifugal blood pumps [69]. Based on similar methods employed by others [53], [67], and to reduce complexity of the simulation, steady laminar flow was simulated for all cases.

The pump geometry was meshed using the structured Cartesian immersed-body method and a mesh independence analysis was conducted using four different meshing schemes (Appendix E, Figure E-1) with increasing levels of cell refinement (coarse to fine) at a flow rate of $Q=1.00 \mathrm{~L} / \mathrm{min}$ and rotational speed of $n=3000$ RPM. Convergence goals for generated pressure $(\Delta \mathrm{P})$ and impeller torque were used to evaluate the strength of each meshing 
scheme (Appendix E, Figure E-2). Meshing Scheme 3 showed less than a $0.50 \%$ difference in converged $\Delta \mathrm{P}$ value and less than a $1 \%$ difference in converged torque value compared to the finest meshing Scheme 4. Therefore, meshing Scheme 3 with 1,155,126 cells was used. All cases were run as timedependent, transient simulations. Average inlet and outlet pressures and velocity as well as average torque (in the rotating axis) of the impeller and $\mathrm{X}, \mathrm{Y}$, and $\mathrm{Z}$ translational forces (summation of forces acting on all impeller body surfaces) were used as convergence goals with $2 \%$ acceptance criteria. All simulations used a variable time-step to a physical flow time of 0.50 seconds or until all goal targets were sufficiently converged.

For comparative purposes, additional CFD simulations of the Inspired Pediatric VAD V3 were conducted assuming blood as a Newtonian fluid and laminar flow as well as a version of the $k-\varepsilon$ turbulence model to solve the Reynolds Averaged Navier-Stokes equations [99]. These results are presented in the Appendix F.

\subsubsection{Shear Stress Analysis}

Blood damage due to flow fields within rotary blood pumps is determined by two factors: the magnitude of the shear stress that the blood is exposed to and the exposure time of the blood to this stress. Hemolysis of red blood cells occurs with a shear stress of $150-250 \mathrm{~Pa}$ coupled with an exposure time of approximately 100 seconds [71]. Additional factors to consider are the degradation of von Willebrand factor (VWF), which has been observed in patients 
with continuous flow VADs [100], and the activation of platelets, which can lead to thrombus formation. In this study, we applied the method presented by Fraser et al. [68] and applied by others [101]-[103] to quantify these factors with three thresholds: (1) shear stress of $9 \mathrm{~Pa}$ for vWF degradation, (2) $50 \mathrm{~Pa}$ for platelet activation, and (3) $150 \mathrm{~Pa}$ for hemolysis. In SolidWorks Flow Simulation, shear rate for non-Newtonian liquids is defined as:

$$
\dot{\gamma}=\sqrt{d_{i j}{ }^{2}-d_{i i} \cdot d_{j j}}, \quad d_{i j}=\frac{\partial u_{i}}{\partial x_{j}}+\frac{\partial u_{j}}{\partial x_{i}}
$$

A scalar representative viscous shear stress $(\tau)$ was calculated in each fluid mesh cell as the product of shear rate and dynamic fluid viscosity $(\mu)$ :

$$
\tau=\mu(\dot{\gamma})
$$

Fluid residence time in each mesh cell was calculated as an estimate of blood exposure time. The size of each cell was estimated by taking the cubic root of the cell volume $(\mathrm{CV})$ and residence time was determined by dividing the cell size by the velocity magnitude $(v)$ [101]:

$$
\text { Residence Time }=\frac{\sqrt[3]{C V}}{v}
$$




\subsubsection{Hydrodynamic Performance}

The hydrodynamic performance of the Inspired Pediatric VAD V3 was characterized by computationally predicting generated pressures $(\Delta P)$ for various flow rates $(Q) 0.50-4.00 \mathrm{~L} / \mathrm{min}$, for three rotational speeds, 3000, 4000, and 5000 RPM. The flow rates and predicted pressures were used to form characteristic pump curves ( $\mathrm{H}-\mathrm{Q}$ curves) and were compared to curves generated from computational analysis of the previous pump version VAD V2.

\subsection{Results and Discussion}

\subsubsection{Hydrodynamic Performance}

VAD V3 was able to achieve pressures of $75 \mathrm{mmHg}$ and $179 \mathrm{mmHg}$ at the lowest operating point of $3000 \mathrm{RPM}(0.5 \mathrm{~L} / \mathrm{min})$ and at the highest at $5000 \mathrm{RPM}$ (4.00 L/min), respectively (Figure 5-4). The predicted pressures for VAD V2 and VAD V3 agreed well with less than a $3 \%$ difference between all predicted corresponding values. Although the rotor body and pump housing of the pump were redesigned for $\mathrm{VAD} V \mathrm{~V}$, the design of the impeller and pump volute were unchanged between $\mathrm{V} 2$ and $\mathrm{V} 3$, resulting in equivalent hydrodynamic performance result. 


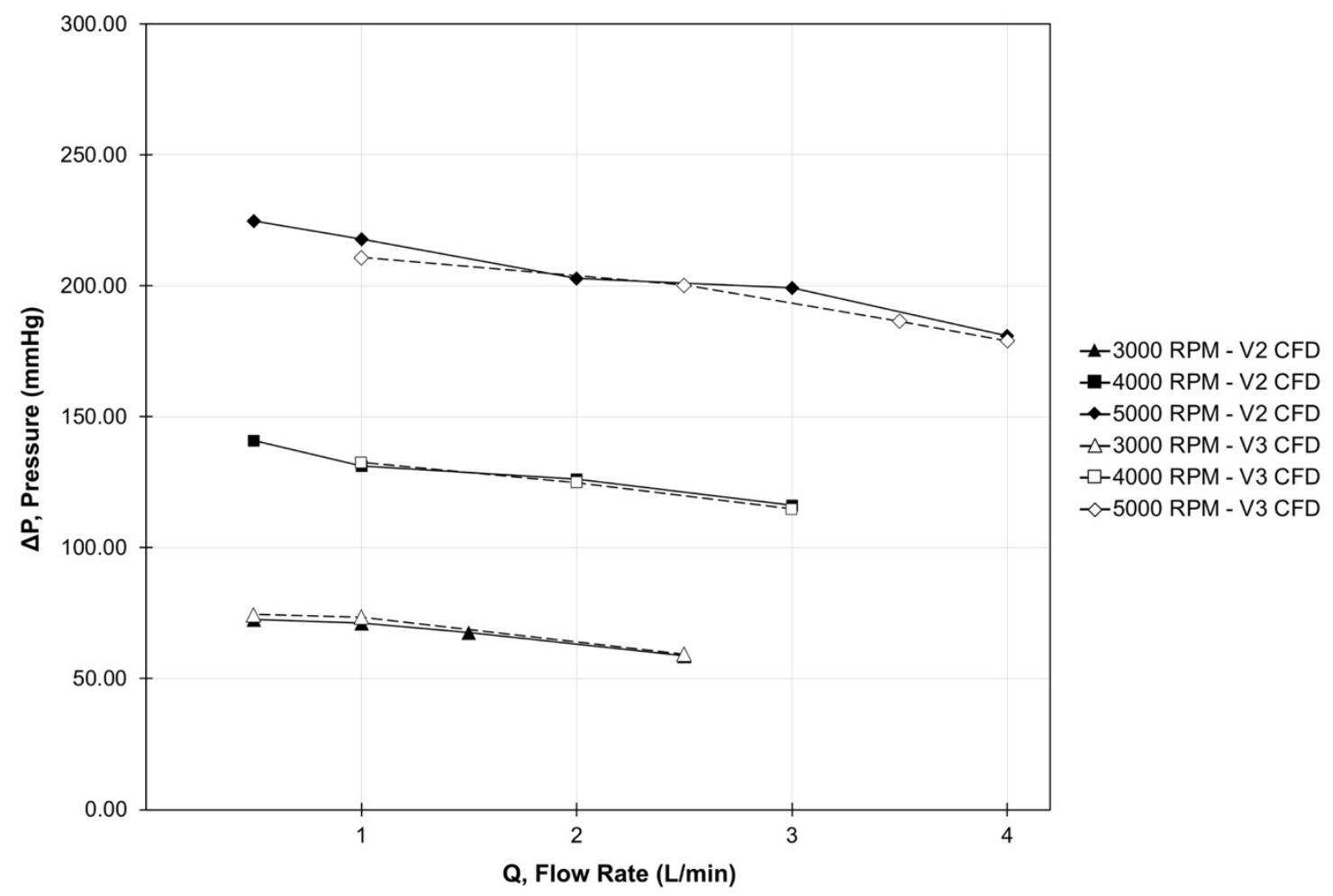

Figure 5-4. Computationally predicted characteristic pressure-flow curves $(\mathrm{H}$ Q) for Inspired VAD V2 and V3 at various rotational speeds (3000, 4000, 5000 RPM) and flow rates $(0.50-4.00 \mathrm{~L} / \mathrm{min})$.

\subsubsection{Impeller Torque and Translational Forces}

With the elongation of the rotor body, the overall surface area of the impeller/rotor increased from $3910 \mathrm{~mm}^{2}$ for $\mathrm{V} 2$ to $8509 \mathrm{~mm}^{2}$ for V3.

Subsequently, the hydrodynamic torque (along the rotational axis) acting on the VAD impeller/rotor significantly increased in VAD V3. Characteristic torque curves for VAD V2 and VAD V3 are shown in Figure 5-5. Peak torque increased from $10.5 \mathrm{mN}-\mathrm{m}$ for VAD V2 to $20 \mathrm{mN}-\mathrm{m}$ for VAD V3 at $5000 \mathrm{RPM}$ and 4.00 $\mathrm{L} / \mathrm{min}$. This increase in torque was expected and incorporated into the MagLev motor design. 


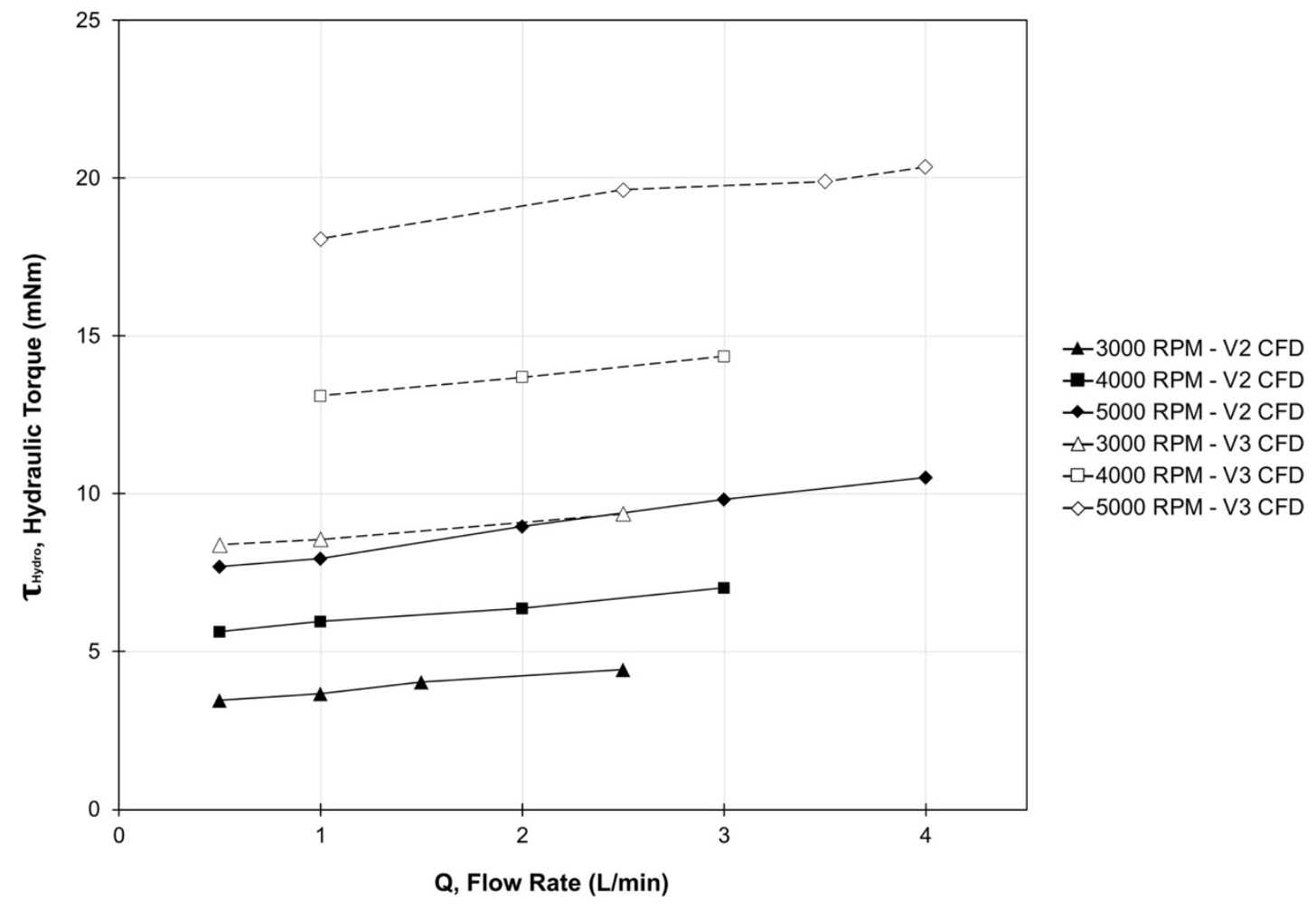

Figure 5-5. Computationally predicted impeller/rotor hydraulic torque values for Inspired VAD V2 and V3 at various rotational speeds (3000, 4000, $5000 \mathrm{RPM})$ and flow rates $(0.50-4.00 \mathrm{~L} / \mathrm{min})$.

Computationally predicted translational hydrodynamic force values for the VAD V3 impeller/rotor in the $x, y$, and $z$ vectors are plotted in Figure 5-6 for comparison to VAD V2. These values were derived as the sum of all force values acting on the surface of the impeller/rotor in each CFD simulation. Tangential forces (X vector) were relatively small $(\leq 0.5 \mathrm{~N})$ and similar for VAD V2 and VAD V3 for all rotational speeds. The axial force ( $Z$ vector) in the positive direction increased with increasing rotational speed, likely due to the lift force created by the impeller rotation, for VAD V2 and VAD V3. VAD V3 attained equivalent or higher axial force values compared to VAD V2 and consistently showed higher axial forces across all flow rates at 5000 RPM. Maximum lift forces increased to 

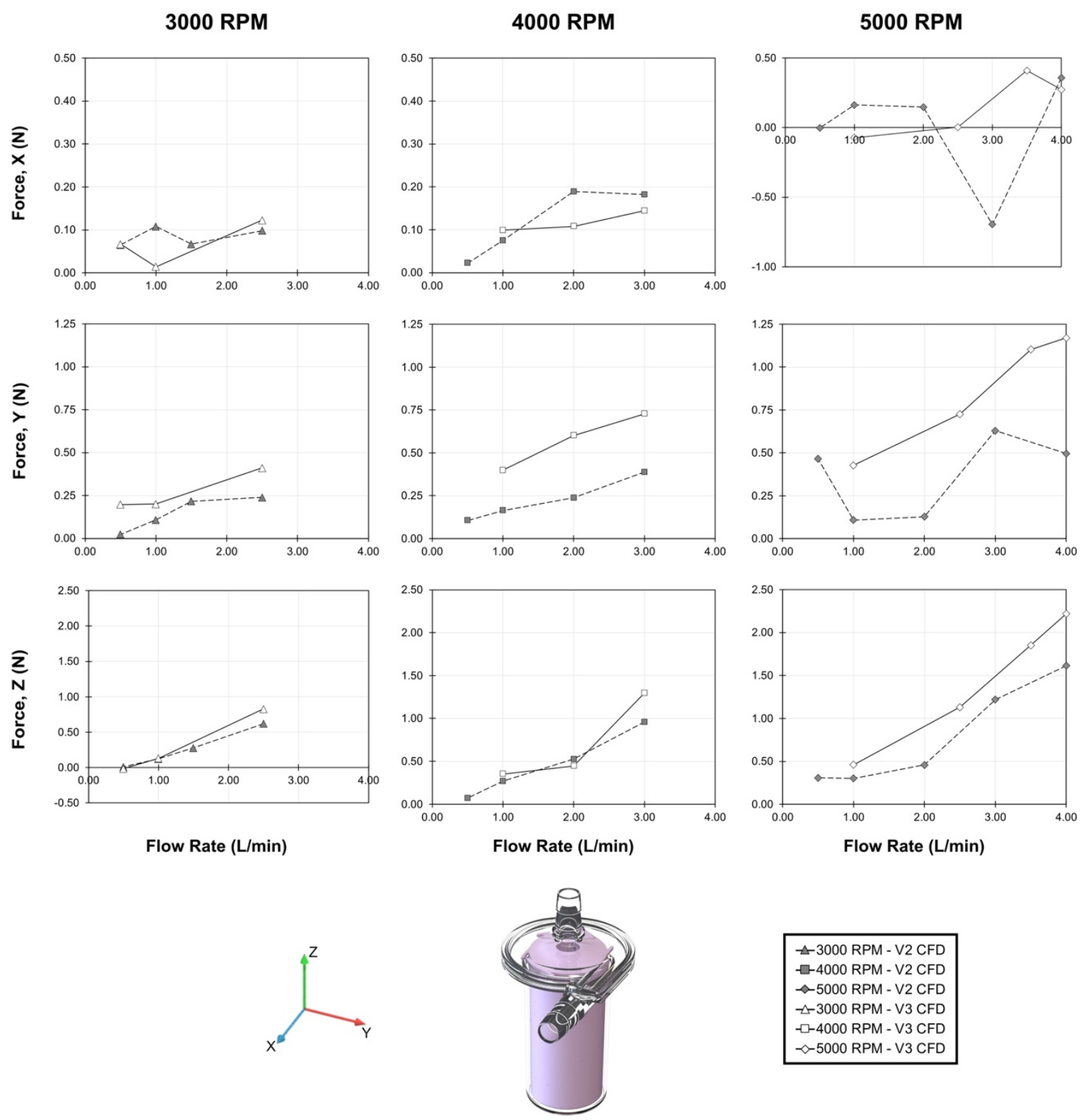

Figure 5-6. Plots of computationally predicted translational $X, Y$, and Z-axis forces of the Inspired Pediatric VAD V2 and V3 impeller/rotor at various rotational speeds (3000, 4000, $5000 \mathrm{RPM})$ and flow rates (0.50 - $4.00 \mathrm{~L} / \mathrm{min})$.

2.2 $\mathrm{N}$ for $\mathrm{VAD} V 3$ from $1.6 \mathrm{~N}$ for $\mathrm{VAD} \mathrm{V} 2$ at $5000 \mathrm{RPM}$ and $4.00 \mathrm{~L} / \mathrm{min}$. Although the main hydrodynamic components were unchanged between $\mathrm{V} 2$ and $\mathrm{V} 3$, additional lift forces may have been caused by an increase in axial blood gap $(0.50 \mathrm{~mm} \mathrm{V2}$ to $0.75 \mathrm{~mm} \mathrm{V3}$ ) and the increased flow through the rotor body 
center opening with the absence of the center post. Differences in meshing schemes and/or fluid modelling between the analyses of VAD V3 and VAD V2 may also have contributed to some discrepancies between force values.

\subsubsection{Pressure Distribution and Flow Fields}

Figure 5-7 shows the pressure distributions through a cross-sectional plane through the top of the impeller blades and the center of the pump outlet tube, and a sagittal plane through the center of the pump inlet tube. The

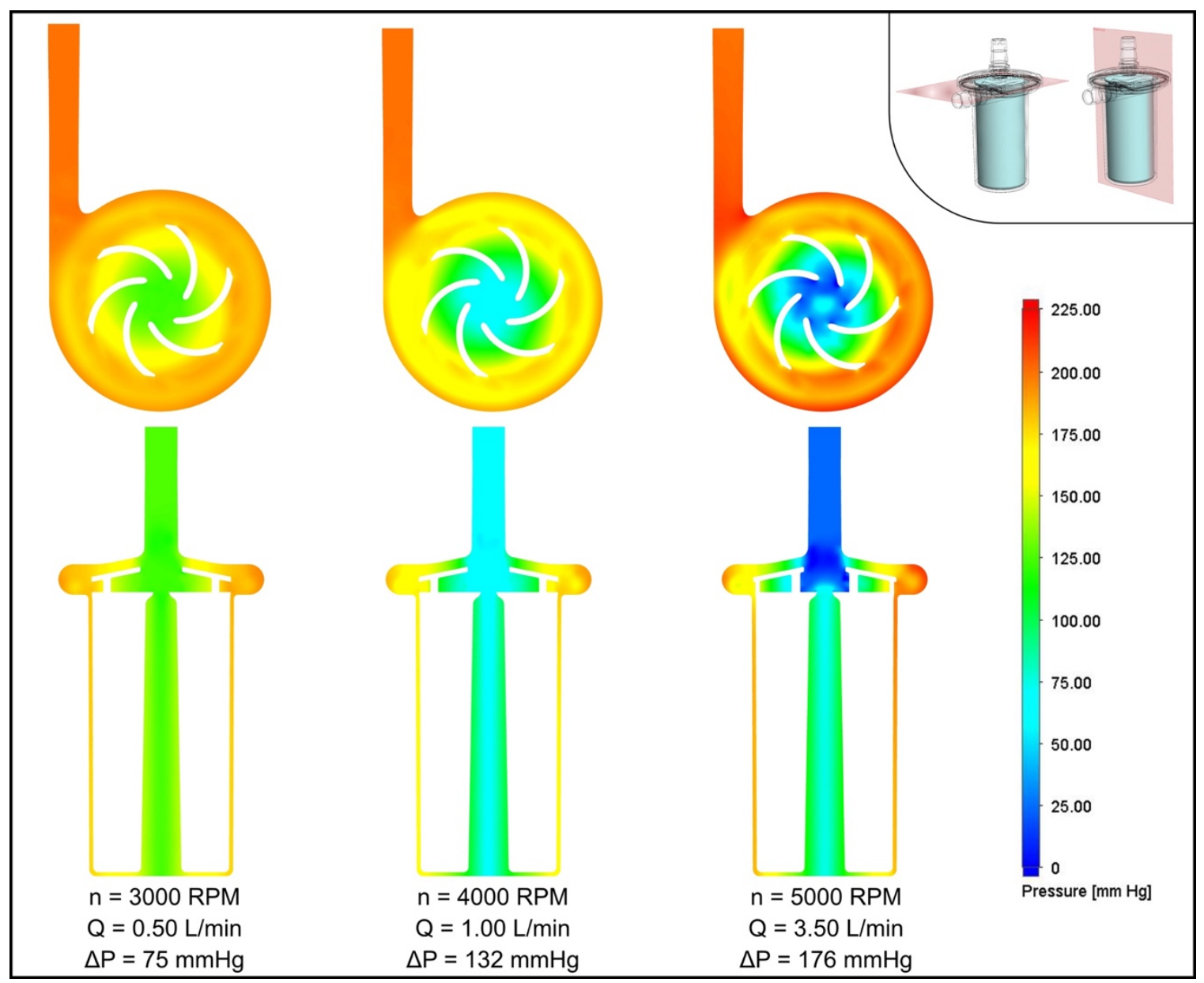

Figure 5-7. Pressure distribution through the impeller blades (top) and planar to pump inlet center (bottom) of the Inspired Pediatric VAD V3 at various rotational speeds (3000, 4000, 5000 RPM) and flow rates (0.50, 1.00, 3.50 L/min). 
boundary condition of a static pressure of $200 \mathrm{mmHg}$ at the pump outlet was maintained for each simulation allowing the pressure difference at the pump inlet $(\triangle P)$ to be generated. VAD V3 was able to achieve a pressure of $176 \mathrm{mmHg}$ across the pump at $5000 \mathrm{RPM}(3.50 \mathrm{~L} / \mathrm{min})$ compared to just $75 \mathrm{mmHg}$ at 3000 RPM (0.5 L/min).

The pressure distribution between the impeller inlet eye and the rotor body center opening differed in VAD V3 at higher rotational speed and flow (5000 RPM and $3.50 \mathrm{~L} / \mathrm{min}$ ), with a $40-50 \mathrm{mmHg}$ difference between the two areas. This differs from similar centrifugal pumps, such as the Thoratec PediMag, which demonstrated a uniform pressure distribution from the pump inlet through the impeller eye and rotor body center opening [53]. This variation may be attributed to the overall length of the VAD V3 rotor body compared to that of the PediMag or the difference in blood gap below the rotor body (VAD V3 has a much thinner gap). This pressure difference was not seen in the computational analysis of VAD V2 and may have contributed to the formation of vortices surrounding the rotor body in VAD V3. Two additional views of the pressure distribution at the pump housing surface for VAD V3 are shown in Figure 5-8.

Figure 5-9 describes the three-dimensional velocity flow fields via cross sectional plots through the middle of the pump inlet across range of rotational speeds $(3000,4000,5000 \mathrm{RPM})$ and flow rates $(0.50,1.00,2.00,3.50 \mathrm{~L} / \mathrm{min})$. At lower rotational speeds and flow rates, there is a significant amount of retrograde flow leakage and pre-rotation that extends upward into the pump inlet, which may be eliminated by increasing flow rate and rotational speed. At all flows and 


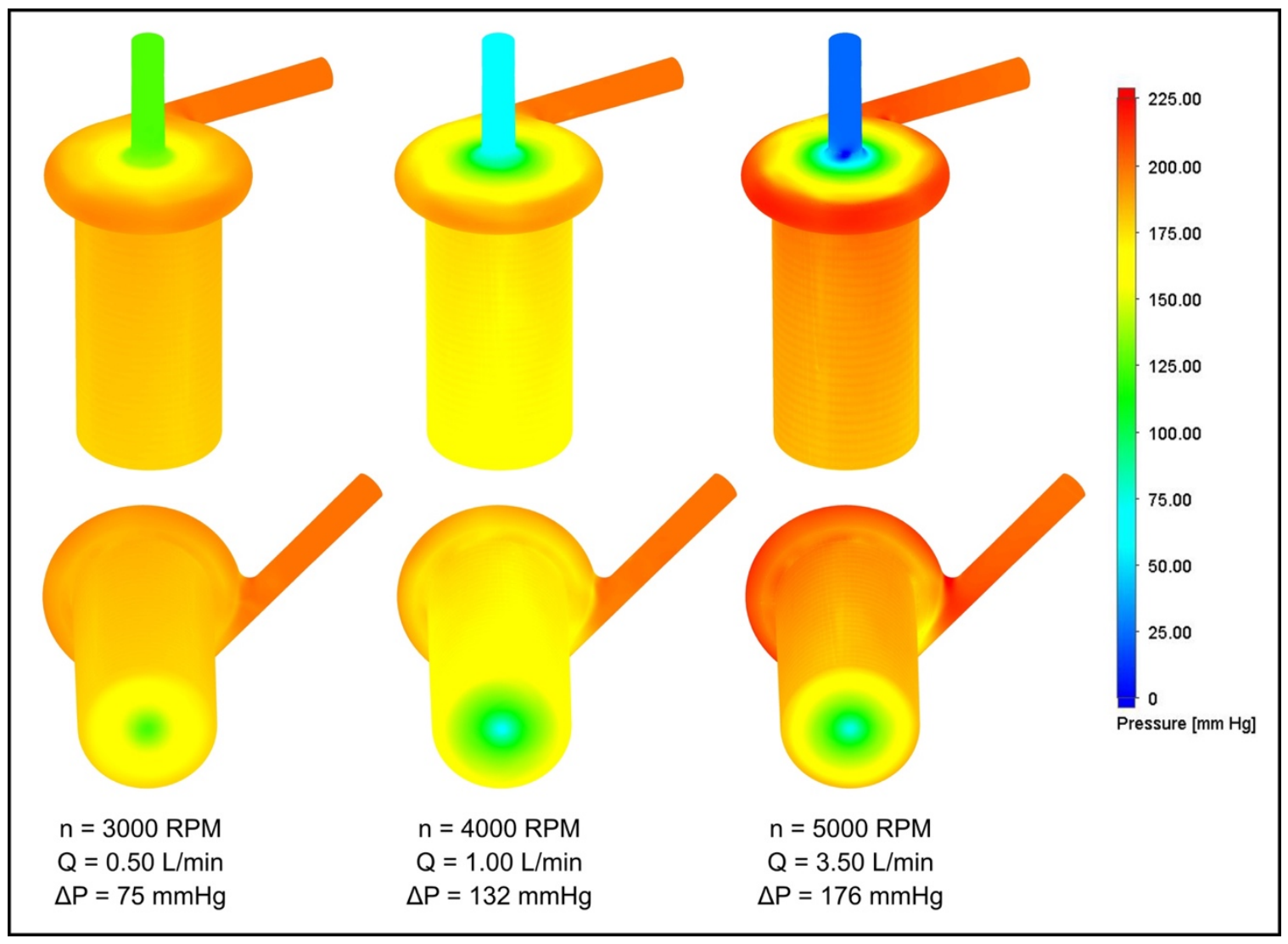

Figure 5-8. Isometric views of pressure distributions at the pump housing surface of Inspired Pediatric VAD V3 at various rotational speeds (3000, 4000, 5000 RPM) and flow rates (0.50, 1.00, 3.50 L/min).

rotational speeds, a uniform vortex is created in the center opening of the rotor body. This differs significantly from the random flow patterns predicted for VAD V1, which also had a center opening within a shorter rotor body that led to the decision to add the center post into the opening in VAD V2 [22]. The results suggested that a center post may not be necessary to promote uniform return flow within the center opening. As was seen in VAD V2, there are three distinct flow paths within the pump in VAD V3: (1) a primary path where flow enters the impeller eye, travels through the blades and into the volute, and exits at the pump outlet (Figure 5-9, shown with white arrows); (2) a secondary flow path 
down around the outside of the rotor housing and then up through the center opening of the rotor housing (Figure 5-9, shown with grey arrows); and (3) tertiary flow at the top of the impeller shroud and the top of the pump housing where flow reverses and travels back toward the jet flow at the pump inlet (Figure 5-9, shown with purple arrows). The secondary and tertiary flow paths can be considered as flow leakages and are detrimental to the overall hydraulic efficiency of the pump, but promote favorable surface washing of all pump surfaces to avoid thrombus formation [41], [42]. 


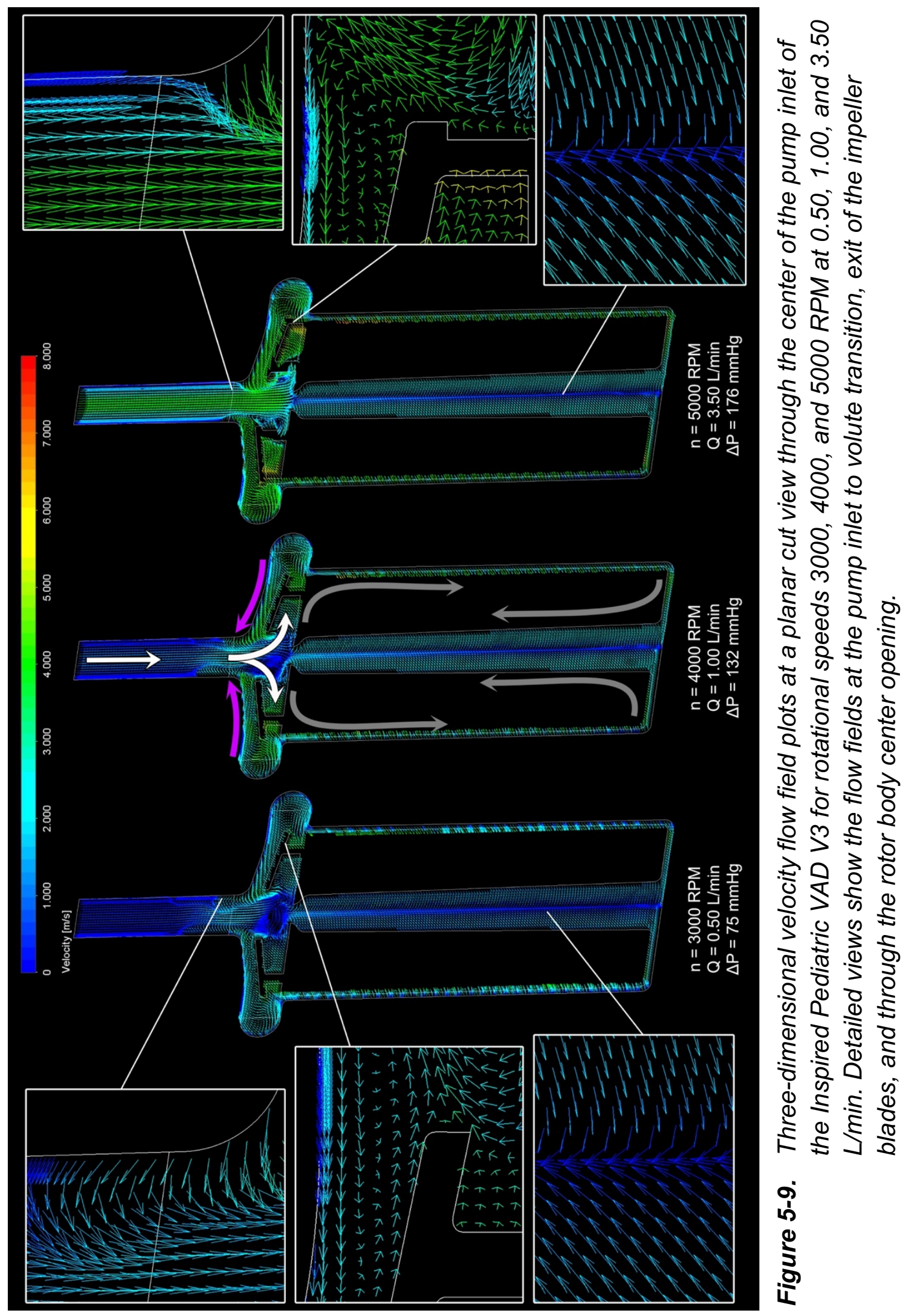




\subsubsection{Taylor Vortices}

An unexpected result of the VAD V3 redesign was the formation of superlaminar Taylor vortices around the circumference of the elongated rotor body. In the previous version, VAD V2 exhibited steady laminar Couette flow in the thin blood gap between the rotor body and the pump housing. In VAD V3, small toroidal vortices known as Taylor vortices appear to form at all rotational speeds and flow rates (Figure 5-10). These vortices form between two concentric cylinders, where the inner cylinder is rotating at a constant speed, typically when the cylinders are long and the gap between them is narrow [104], as is the case of VAD V3. At a critical rotational speed, the centrifugal forces of the inner cylinder exceed the viscous forces of the fluid and the pressure gradient across the cylinder gap, causing the vortices to form. These vortices are still in the laminar regime, considered a type of super-laminar flow, but can transition to completely turbulent vortex flows at high rotational speeds [105]. These vortices may have a negative impact on the design of the VAD V3. The vortices increase the flow or residence time of fluid around the rotor body creating a longer path through the secondary flow, i.e. the time for the fluid particles to travel around the rotor body and back through the center opening will be prolonged compared to the speed traveling through a steady laminar Couette flow [106]. This in turn increases the amount of time blood is exposed to the pump surfaces and potential exposure to higher shear stresses. Further, the vortices can cause areas of increased shear stress and increase the torque of the rotor body [107]. Further work will need to be completed to mitigate or control the formation of 
these vortices, potentially through changing the surface profile of the rotor body or lower pump housing [108]. Appendix E, Figure E-3 details how the vortices form around the rotor body during the transient CFD simulation from 0.010 to 0.50 seconds of flow time.

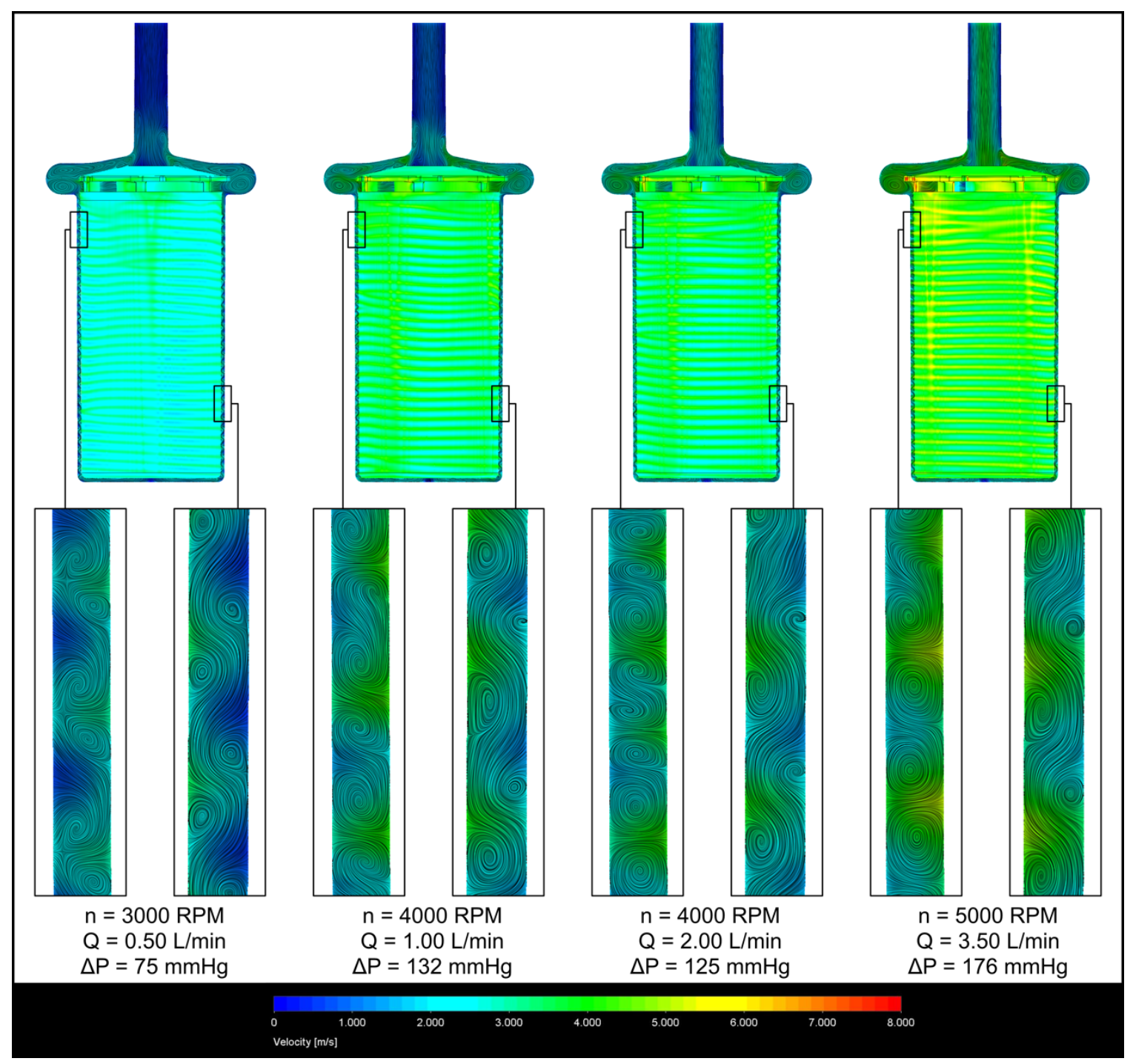

Figure 5-10. Velocity plots of the impeller/rotor surface and surrounding flow in a cut plot planar to the pump inlet center opening with black iso-flow lines displayed to show detail of Taylor vortices formation at various flow rates $(0.50$ to $3.50 \mathrm{~L} / \mathrm{min})$ and rotational speeds $(3000,4000$, 5000 RPM). 


\subsubsection{Shear Stress and Residence Time}

Figure 5-11 shows shear rate distributions at a planar cut plot through the center of the pump inlet. Most of the fluid domain is subjected to low shear rates (less than $10,000 \mathrm{~s}^{-1}$ ) with areas of higher rates at the impeller/rotor and pump housing surfaces. The largest concentrations of higher shear rates appear on the surfaces of the rotor body and lower pump housing at the thin blood gap area. The formation of Taylor vortices in the rotor body/lower pump housing blood gap create concentrated areas of higher shear rates (Figure 5-11). Other concentration areas for higher shear rates include the pump volute surfaces, impeller surfaces, and at the top of the pump housing, which may be attributed to the tertiary flow path in this area caused by the impeller shroud. For all cases, shear rates at the pump surface increase with increased rotational speed and flow rate. Additional shear rate plots are shown in Figure 5-12. 


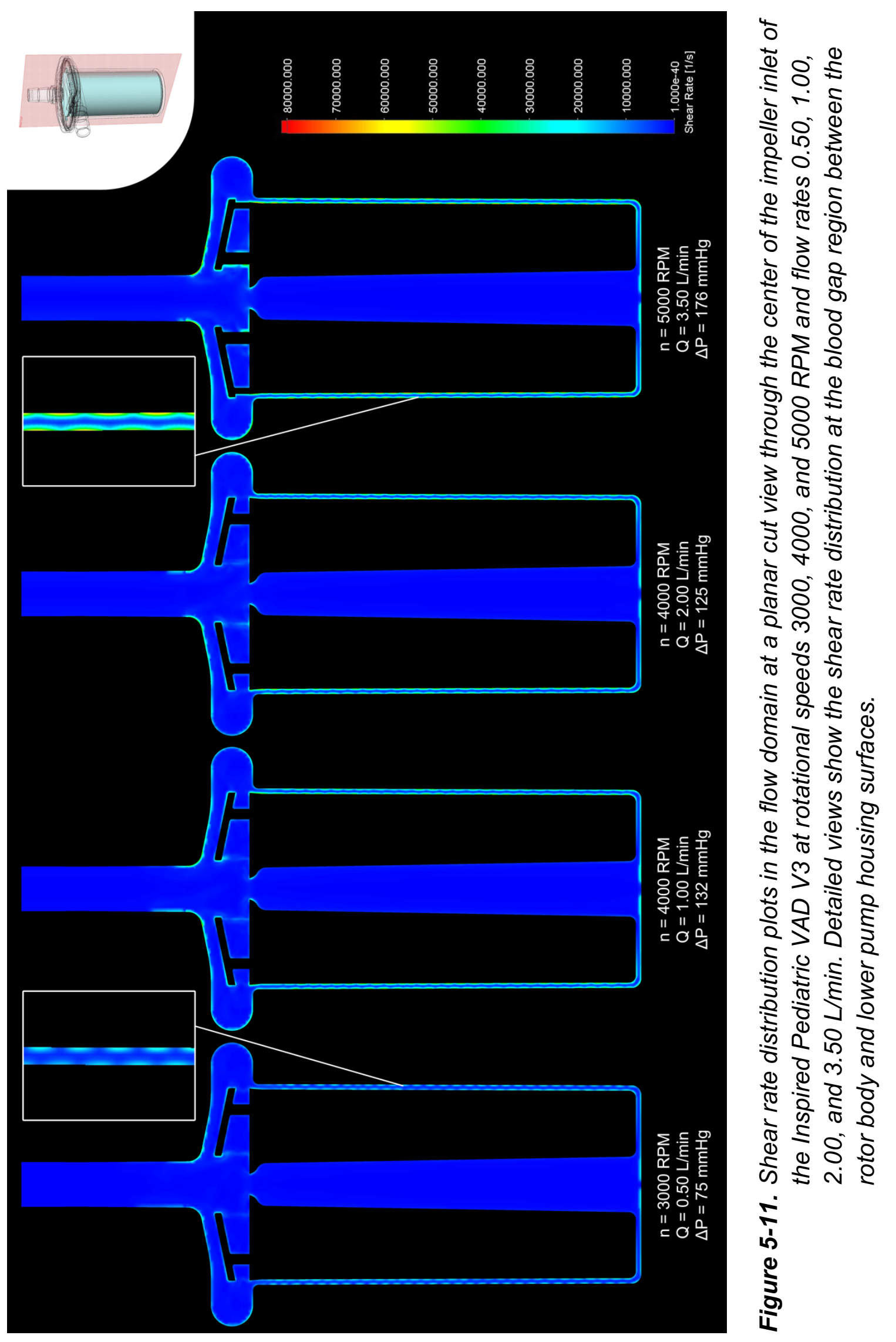




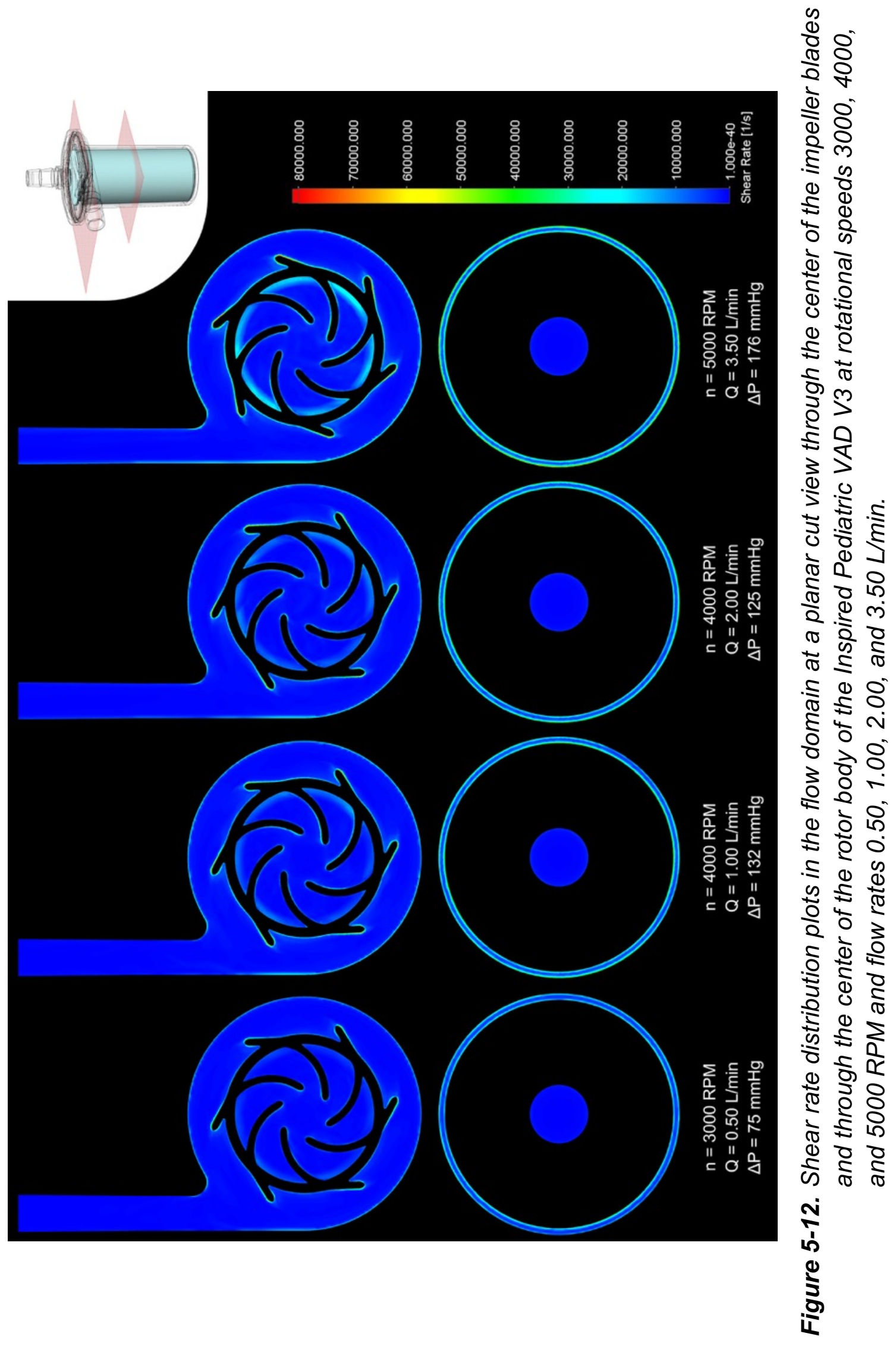


Figure 5-13 shows wall shear stress distribution plots of the impeller/rotor and pump housing surfaces. Concentrations of high shear stress are seen at the impeller blade extension tips and around the rotor body and pump housing in the blood gap region. Even at lowest operating point, $3000 \mathrm{RPM}$ at $0.50 \mathrm{~L} / \mathrm{min}$, there are small areas in these regions that exceed the critical value of $150 \mathrm{~Pa}$ for hemolysis. At the highest operating point, $5000 \mathrm{RPM}$ at $3.50 \mathrm{~L} / \mathrm{min}$, shear stresses in these areas increase significantly with select spots exceeding $450 \mathrm{~Pa}$. The maximum shear stresses generated at the impeller/rotor surface and the pump housing surface were $752 \mathrm{~Pa}$ and $759 \mathrm{~Pa}$, respectively, at $5000 \mathrm{RPM}$ at 3.50 L/min and $176 \mathrm{mmHg}$. A similar computational study of the HeartWare HVAD and Thoratec HeartMate II generated maximum wall shear stresses of $2198 \mathrm{~Pa}$ and $2018 \mathrm{~Pa}$, respectively, at $5.00 \mathrm{~L} / \mathrm{min}$ and $70 \mathrm{mmHg}$ [102]. Although these values are from a higher flow rate, this comparison is encouraging. In addition, the maximum shear stress generated in the flow domain for the HVAD was $3440 \mathrm{~Pa}$ and $1559 \mathrm{~Pa}$ for the HeartMate compared to $1777 \mathrm{~Pa}$ for the Inspired Pediatric VAD V3. 


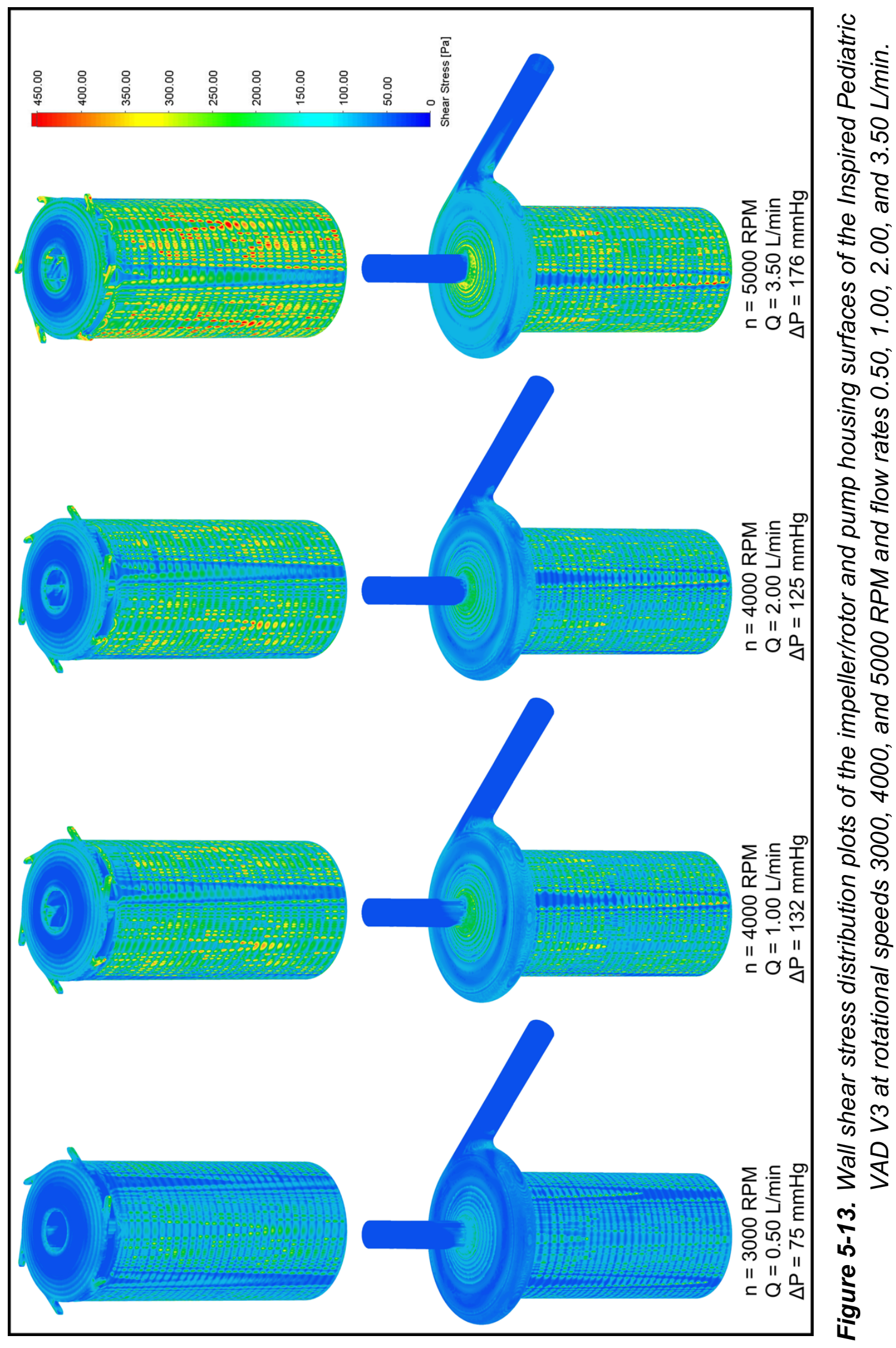


Histograms of the volumetric shear stress distribution and the residence time shear stress distribution are shown in Figure 5-14. For all rotational speeds and flow rates, the majority of pump fluid volume (more than $50 \%$ ) is in the shear stress range of $0-10 \mathrm{~Pa}$. As the rotational speed and flow rate increases, the volumetric distribution of the histograms shift slightly to the right, indicating that more flow is exposed to higher shear stresses at these elevated conditions. $79 \%$
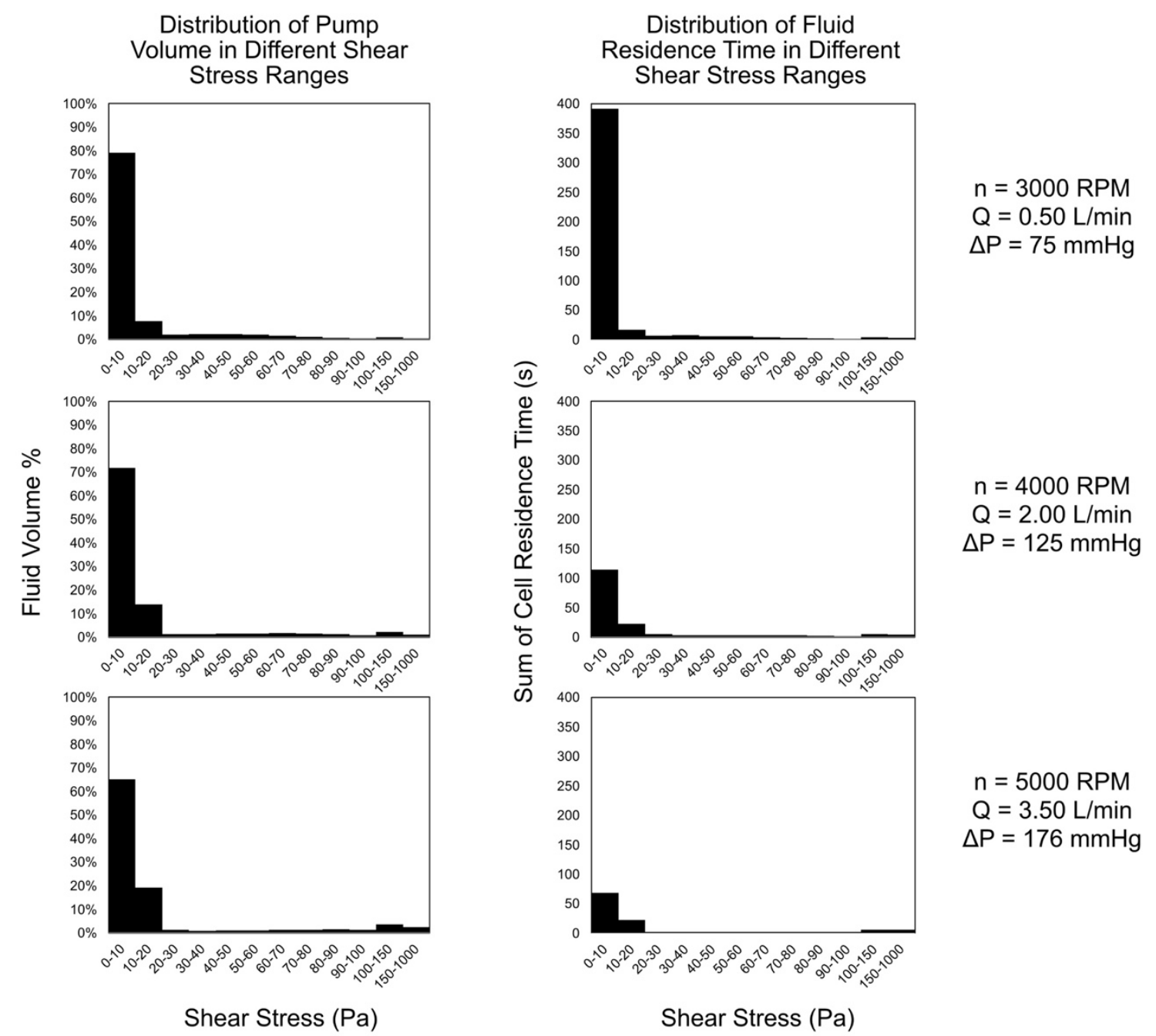

Figure 5-14. Histograms of the volumetric distribution of shear stress (Left) and the residence time distribution of shear stress (Right) of the Inspired Pediatric VAD V3 at rotational speeds of 3000,4000 , and $5000 \mathrm{RPM}$ and flow rates of $0.50,2.00$, and $3.50 \mathrm{~L} / \mathrm{min}$. 
of the volume is in the range of $0-10 \mathrm{~Pa}$ for $3000 \mathrm{RPM}$ at $0.50 \mathrm{~L} / \mathrm{min}$ compared to $65 \%$ for $5000 \mathrm{RPM}$ at $3.50 \mathrm{~L} / \mathrm{min}$. At the higher end of shear stresses, $<1 \%$ of the volume is in the $150-1000 \mathrm{~Pa}$ range for $3000 \mathrm{RPM}$ at $0.50 \mathrm{~L} / \mathrm{min}$ compared to $2.3 \%$ for $5000 \mathrm{RPM}$ at $3.50 \mathrm{~L} / \mathrm{min}$. Still, even at $3.50 \mathrm{~L} / \mathrm{min}$ (the target design point for the Pediatric VAD), $94 \%$ of the volume in the flow domain is exposed to shear stresses at or below 100Pa. The highest residence times within the VAD occur at the lowest flow rate, $0.50 \mathrm{~L} / \mathrm{min}$, and the overall summation of residence times greatly decreases as the flow rate is increased. As with the volumetric distribution, the majority of residence time for all rotational speeds and flow rates resides at or below $100 \mathrm{~Pa}$.

Figure 5-15 shows histograms of volume and residence shear stress distribution considering the critical thresholds of 9,50 , and $150 \mathrm{~Pa}$. The highest operating point of $5000 \mathrm{RPM}$ at $3.50 \mathrm{~L} / \mathrm{min}$ has the highest volume percentage for each threshold, with approximately $2.3 \%(3.4 \mathrm{~mL})$ above the threshold for hemolysis (150 Pa). This compares to $0.20 \%(0.025 \mathrm{~mL})$ for the HVAD and $0.40 \%(0.031 \mathrm{~mL})$ for the HeartMate II [101], [102]. In another similar computational study, Fraser et al. found that the Thoratec CentriMag and UltraMag pumps, centrifugal pumps similar to the Inspired Pediatric VAD V3, both had minimal volume $(<0.5 \%)$ above the $150 \mathrm{~Pa}$ threshold, at a flow rate of 3 L/min [68]. Summed mesh cell residence times above the critical $150 \mathrm{~Pa}$ threshold are low, with all rotational speeds and flow rates below 6 seconds, which is encouraging. Average mesh cell residence times are low $(<7.00 \mathrm{E}-04$ seconds) for all flow rates, with the highest times residing in the neutral shear 

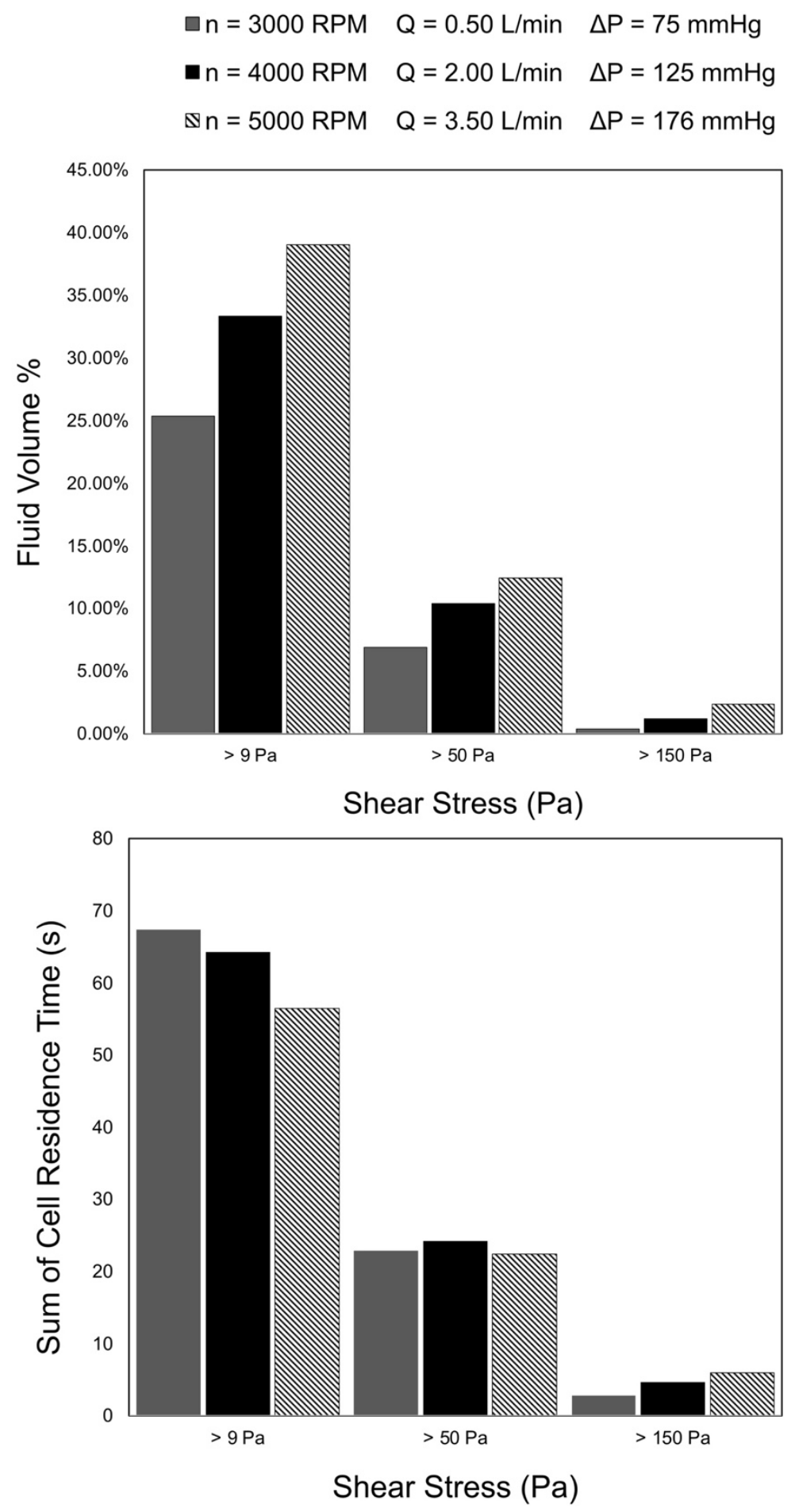

Figure 5-15. Histograms of the volumetric distribution of shear stress (Top) and the residence time distribution of shear stress (Bottom) of the Inspired Pediatric VAD V3 at critical thresholds of $9 \mathrm{~Pa}$ (VWF damage), $50 \mathrm{~Pa}$ (platelet activation), and $150 \mathrm{~Pa}$ (hemolysis). 
stress range of $0-9 \mathrm{~Pa}$. These residence time values compare favorably with the CentriMag and UltraMag for all shear stress thresholds [68]. A plot of average cell residence times is shown in Appendix E, Figure E-4.

In blood pumps, areas of flow stagnation or recirculation, especially at the pump surfaces, can become prone to thrombus formation and platelet aggregation [109], [110] and surface areas with shear rates below $250 \mathrm{~s}^{-1}$ have been shown to have clot formation [111]. Figure 9 shows iso-surface plots detailing areas within the fluid volume where shear rates are below $250 \mathrm{~s}^{-1}, 100$ $\mathrm{s}^{-1}$, and flow velocities are below $0.20 \mathrm{~m} / \mathrm{s}$. A velocity threshold of $0.20 \mathrm{~m} / \mathrm{s}$ was chosen to visually display areas where flow stagnation and recirculation may occur. For all rotational speeds and flow rates, there are low velocity regions in the pump inlet and pump outlet mainly towards the boundary layer of the fully developed flow, with areas diminishing as the flow rate is increased. At flow rates of 0.50 and $1.0 \mathrm{~L} / \mathrm{min}$, small amounts of low velocity may be seen in the center of the impeller eye. Appendix E, Figures E-5 and E-6 show directional velocity vectors in the pump outlet region. Some areas of flow recirculation and flow retrograde do exist at lower flow rates 0.50 and $1.0 \mathrm{~L} / \mathrm{min}$ but these areas disappear at $2.0 \mathrm{~L} / \mathrm{min}$ or greater. There are significant areas that experience shear rates less than $250 \mathrm{~s}^{-1}$ in the pump inlet, outlet, in the center opening of the rotor body, and in the center of the impeller blade channels. Areas of low shear within the pump inlet, outlet, and impeller blade channels diminish as flow rate is increased, with the area within the rotor body center opening remaining. Although significant areas are present, especially at $3000 \mathrm{RPM}$ and $0.50 \mathrm{~L} / \mathrm{min}$, the 
majority of volume is in the center of the flow channels for each of these regions and are not in contact with any pump surfaces. Only surface areas experiencing low shear rates should be concerning for clot formation. Blood at shear rates

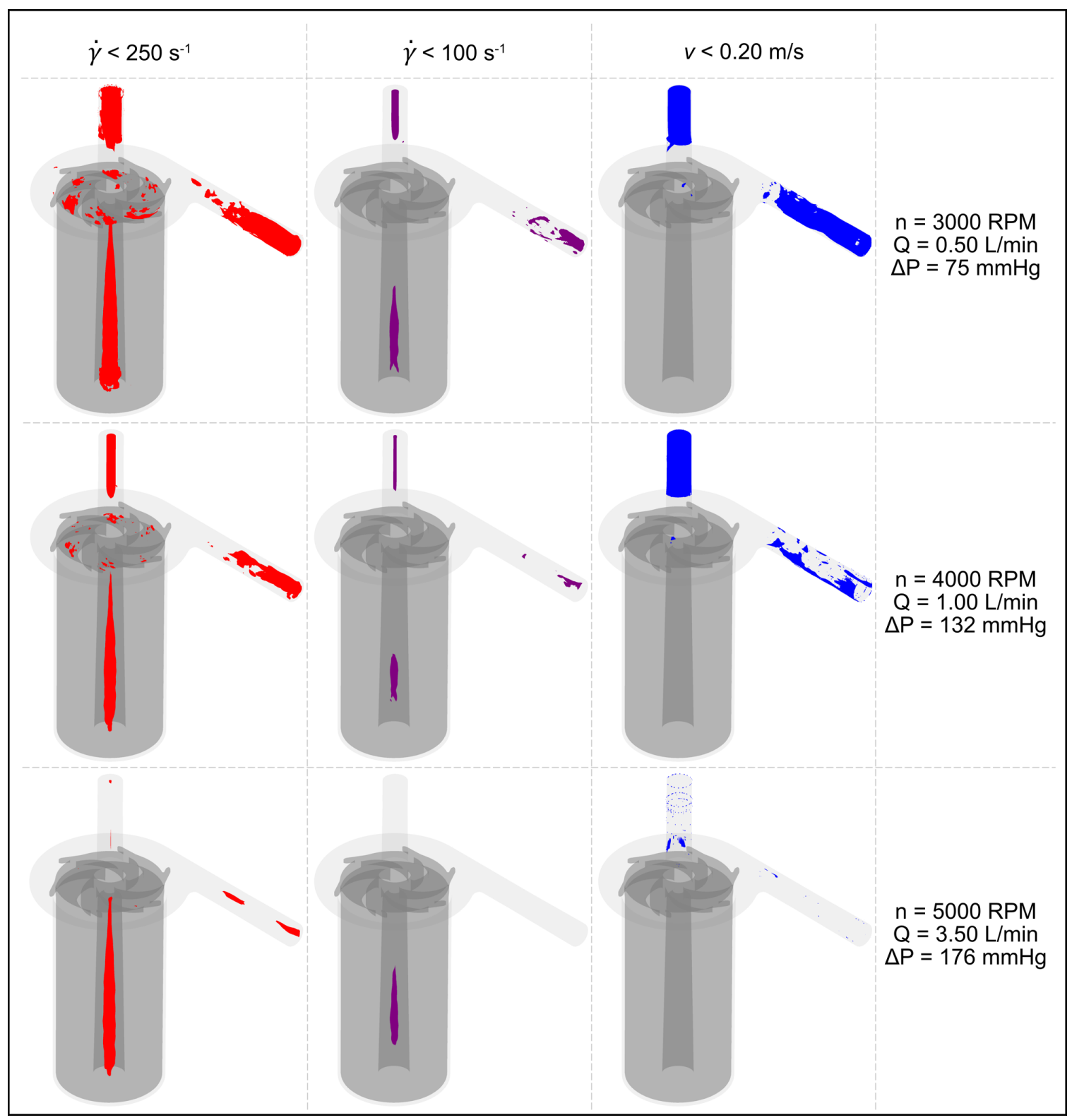

Figure 5-16. Summary of iso-surface plots detailing areas within the fluid volume where shear rates are below $250 \mathrm{~s}^{-1}$ (possible clot formation at pumping surfaces), $100 \mathrm{~s}^{-1}$ (areas where blood behaves as a nonNewtonian fluid), and flow velocities are below $0.20 \mathrm{~m} / \mathrm{s}$ (areas of possible flow stagnation and recirculation), for various rotational speeds and flow rates. 
above $100 \mathrm{~s}^{-1}$ behaves as an incompressible Newtonian fluid, so areas below this threshold are of interest from a fluid modeling perspective.

\subsubsection{Study Limitations}

The Inspired Pediatric VAD V3 is the next iteration of a novel device that has been developed based on an evolution of a variety of different prototype versions; however, the simulations and numerical predictions presented in this study have not yet been validated empirically with a VAD V3 prototype. The MagLev motor system and controller have been fully evaluated, with next steps involving evaluation of these components coupled with the VAD V3 pump design. Full bench testing in a mock circulatory loop and acute animal implantation are planned for the next development phase of this project.

Although SolidWorks Flow Simulation is a powerful CFD tool with many unique facets, it does not provide the implementation of user defined variables and/or scalars in the solver portion of the software. Due to these limitations, a full picture of the VAD V3's hemocompatibility could not be completed in this study. Although shear stress, shear rate, and residence time distributions are useful indicators for blood damage, estimations of the distribution of plasma-free hemoglobin using a Eulerian transport equation and hemolysis index $(\mathrm{HI})$ using a power-law model have been employed by others in different software packages [68], [101], [112], [113]. A full characterization of these variables in the Inspired Pediatric VAD V3 may be useful in comparison to other published blood pump designs and will be investigated in future studies. 


\subsection{Conclusion}

In this study, CFD simulations were used to characterize the novel Inspired Pediatric VAD V3. Numerically predicted hemodynamic performance and volumetric shear stress and fluid residence times were presented as well as an evaluation of the wall shear stress distributions across the impeller/rotor and pump housing surfaces. The phenomena of Taylor vortices were observed due to the new elongated design of the rotor body around its perimeter for all rotational speeds and flow rates. Areas of low shear rates and low flow velocities were observed in the pump outflow channel which will require further investigation as it pertains to potential clot formation.

Future development efforts include full characterization of performance and safety of the Inspired Pediatric VAD V3, in vitro testing in static and dynamic mock circulatory loop models and in vivo testing in acute and chronic large animal studies. Our ultimate goal is to translate this new device into clinical practice, which may greatly benefit pediatric HF patients who remain in dire need of a dedicated MCS solution. 


\section{CHAPTER 6 : CONCLUSIONS AND FUTURE WORK}

\subsection{Conclusion}

Through the research and development efforts described in this dissertation, the aim for this project was achieved as evidenced by completion of the design, development, and evaluation of the impeller and pump housing components of the Inspired Pediatric VAD through three progressive iterations (VAD V1, V2 and V3). The last design V3 integrated the MagLev motor system, achieved desired pump operational parameters (flows of $0.5-3.5 \mathrm{~L} / \mathrm{min}$ at pressures of $40-150 \mathrm{mmHg}$ ), and exhibited promising hemocompatibility predictions.

\subsubsection{Impeller and Pump Design}

Established turbomachinery calculation and dimensional analysis techniques were leveraged to formulate the impeller and pump housing designs of the Inspired Pediatric VAD. These traditional approaches, which are intended for much larger industrial pump applications, were combined with data and knowledge gleaned from past and current rotary blood pump development efforts. 
Since VADs are a new and evolving technology, established design theory is not readily available. Using a combination of traditional calculations and VAD specific elements, a hybrid design approach was developed and tailored specifically for the Inspired System. This approach was used to establish the basic design of the Inspired Pediatric VAD and will be used in future development efforts to design additional single-use pumps (neonate, adult, pulmonary support). This design approach was integrated into a spreadsheet to

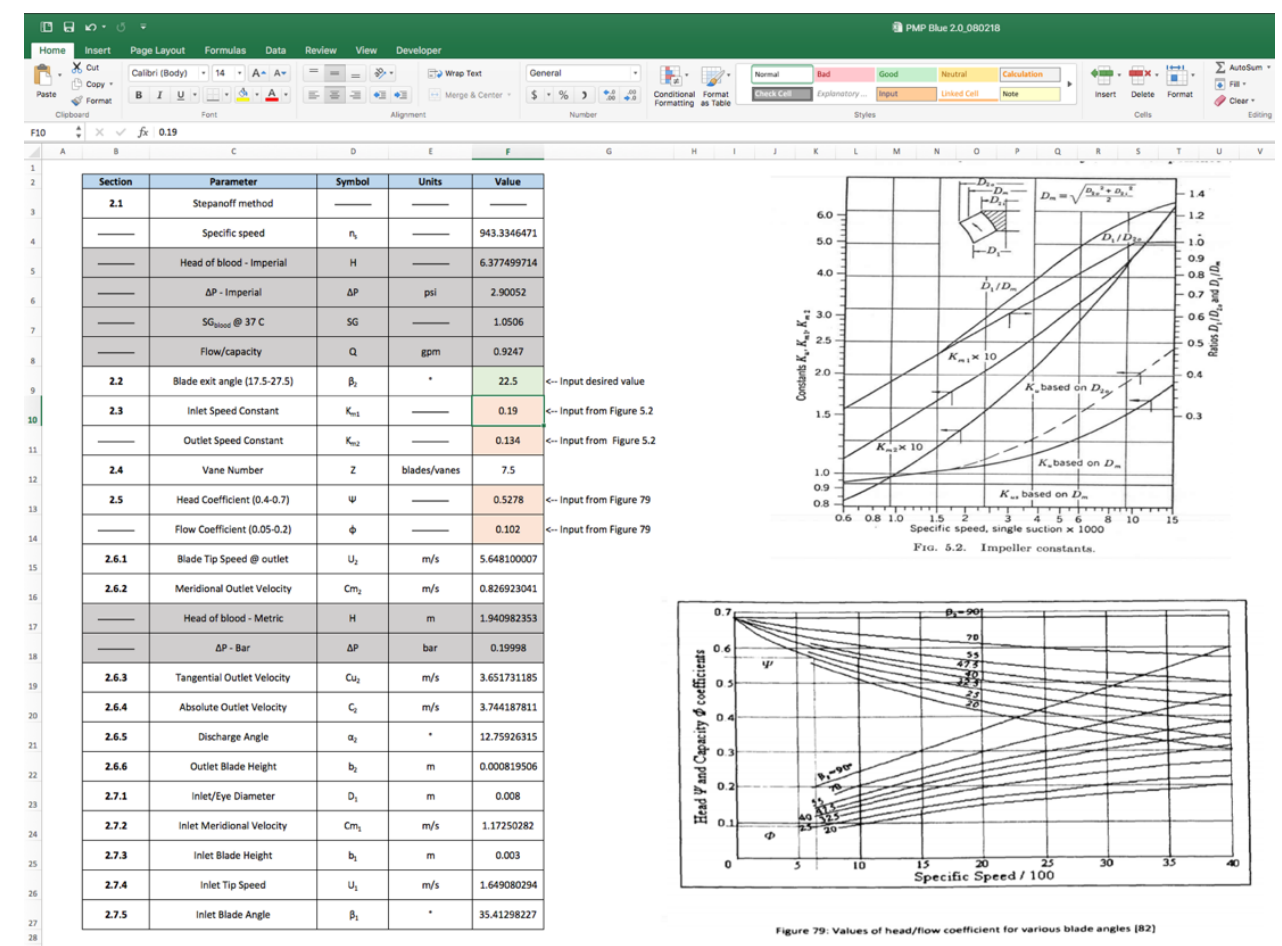

Figure 6-1. Example of dimensionless constants calculations in the Inspired Pump Design Program.

accelerate the process and to facilitate the development of future impeller design iterations (Figures 6-1, 6-2). 


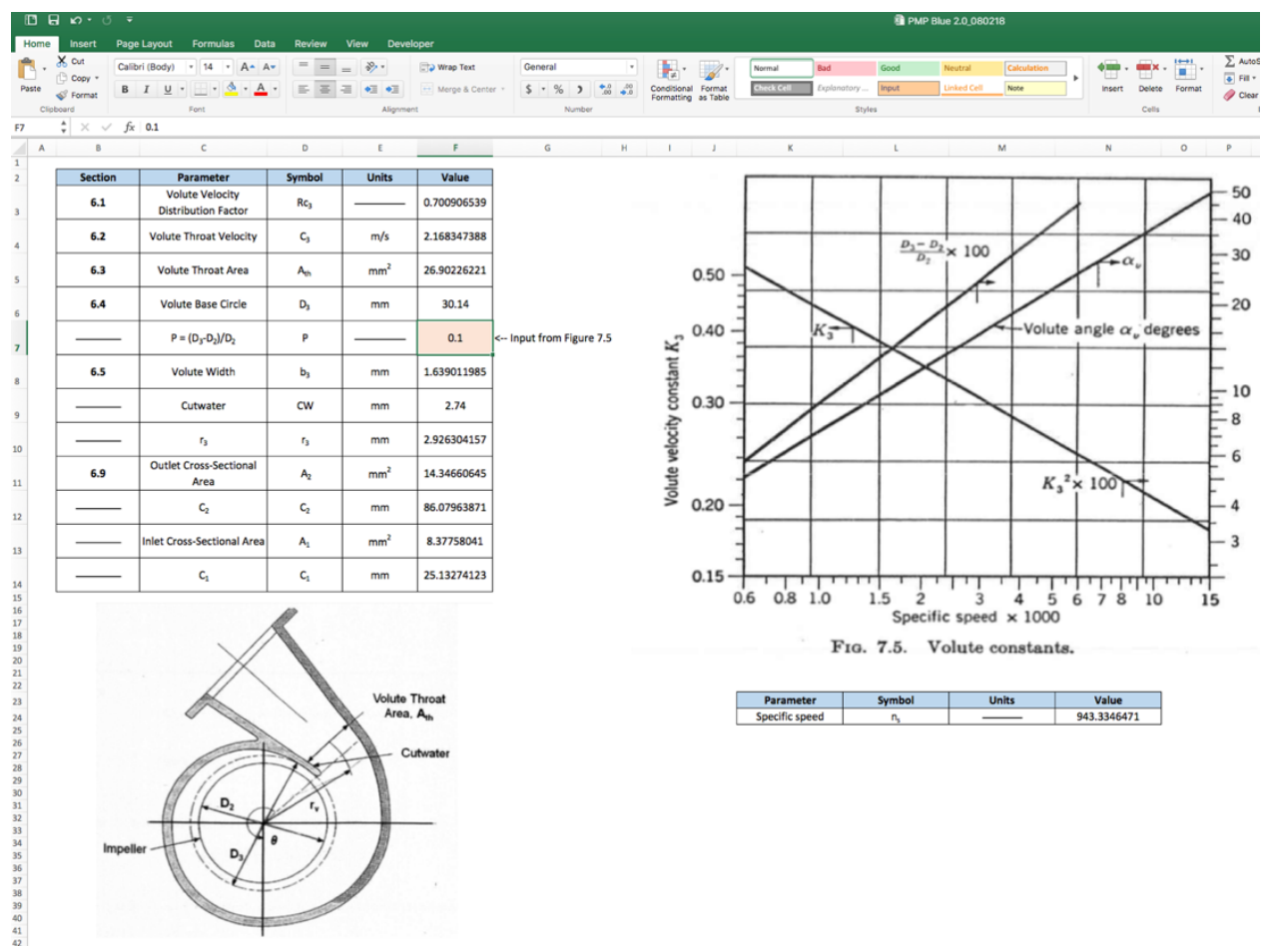

Figure 6-2. Example of volute dimensions calculations in the Inspired Pump Design Program.

\subsubsection{CFD Simulation}

Computational fluid dynamics (CFD) models were constructed for each Inspired Pediatric VAD design iteration. Simulations were conducted for each design, resulting in hydrodynamic, hemodynamic, and torque/force data that informed iterative design decisions. Additionally, data from these simulations were used in the design of the MagLev motor for the Inspired Universal MagLev system. Key engineering design requirements of the Inspired Pediatric VAD were identified through CFD simulations including: the need for a center opening through the rotor body to counter-act axial lift forces generated by the impeller; the need for impeller blade extensions to induce uniform flow in the entire pump 
volute; the possible need for a center post to promote uniform return flow through the rotor body center opening; and the formation of Taylor vortices along the radial blood gap. Additionally, areas of high and low shear as well as low flow velocity were identified for future design improvement.

\subsubsection{Prototype Testing}

A shaft driven Inspired Pediatric VAD V2 was constructed and successfully tested in a static flow loop model. Important hydrodynamic and impeller hydraulic torque data were generated from this study. These data were utilized in the design of the MagLev motor system. Additionally, the processes and protocols developed during this study will be used in future VAD prototype bench testing efforts.

\subsubsection{Final Design}

A final Inspired Pediatric VAD geometry, VAD V3, was created, integrating the novel MagLev motor into the design, and was evaluated through CFD simulations and hemodynamic analysis. The geometry of the VAD V3 design is unique, with an elongated rotor body and relatively small impeller diameter. The design was predicted to achieve the desired flow and pressure requirements. Hemodynamically, the design compares closely with current clinically available devices, with most of the flow volume below the critical shear stress threshold of $150 \mathrm{~Pa}$, although further analysis is required. Prototype VAD V3 devices are currently being fabricated (Figure 6-3), using a mixture of injection-molded and 
additive manufacturing components for precise production of the parts with the optimal geometry, and will be tested in the next phase of this project.

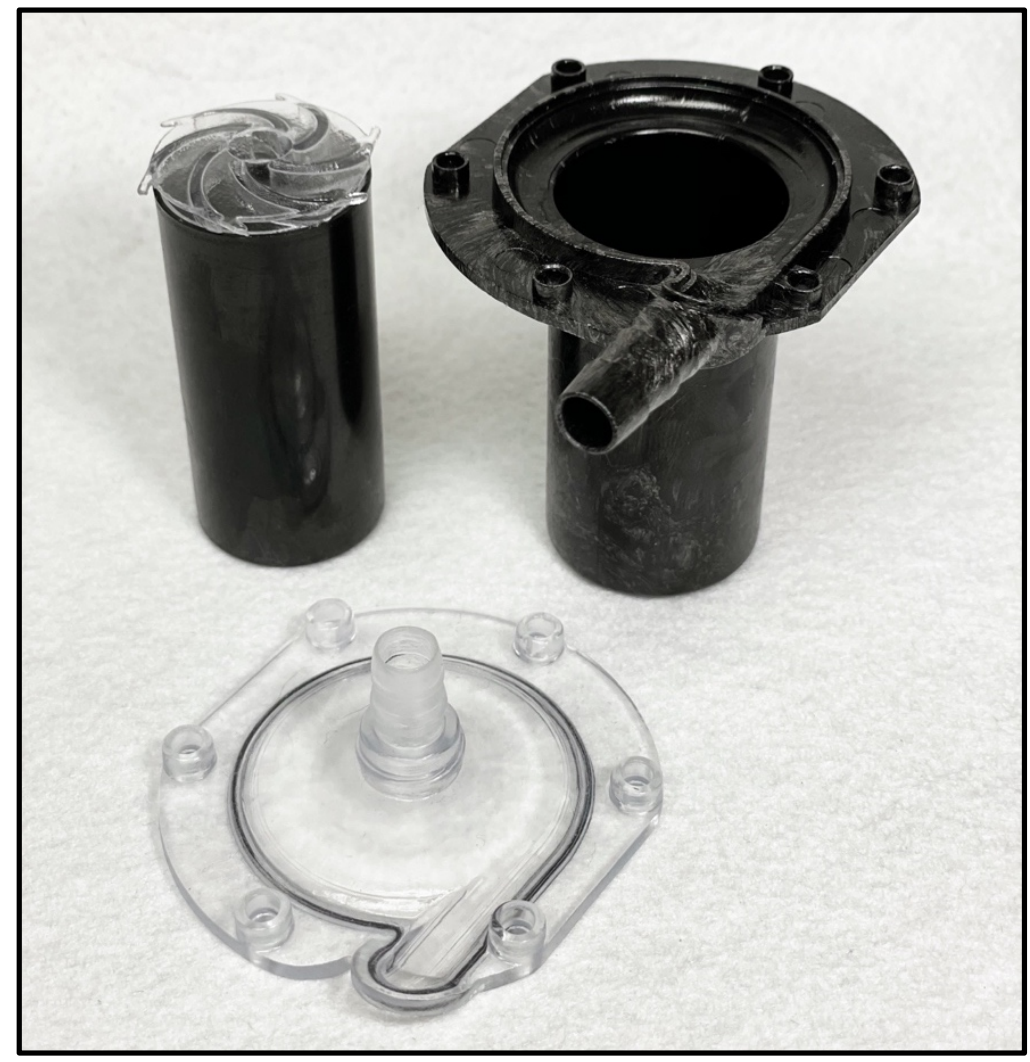

Figure 6-3. Injection molded and additive manufacturing components of the Inspired Pediatric VAD V3 prototype currently under development.

\subsection{Future Work}

Although a large development effort has been completed in the Inspired Pediatric VAD project, there are additional outstanding engineering challenges to be addressed as the pump design progresses towards the final clinical-grade version. Future research and development work being carefully considered is presented next. 


\subsubsection{In Vitro Mock Circulatory Loop Testing}

To complete the first phase of the Inspired Pediatric VAD project, in vitro bench testing and in vivo acute large animal experiments are planned to evaluate the VAD V3 design. Bench testing will be performed in a pediatric dynamic mock flow loop model simulating a $20-\mathrm{kg}$, three-year-old pediatric patient. The pediatric mock flow loop model consists of a pneumatically driven silicone ventricle, venous return reservoir, and systemic vasculature components. The loop will be used to characterize the hemodynamic performance of VAD V3. The mock flow loop model has a proximal resistance (afterload), an elevated storage element (preload), compliance element, and a distal resistance, which can be tuned to simulate pediatric pressures and flows for normal, heart failure, hypertension, hypotension, hypovolemia, and hypervolemia test conditions. The hemodynamic performance will be characterized over the entire operational range and compared for each baseline test condition. VAD V3 will be tested in both a static loop condition, for $\mathrm{H}-\mathrm{Q}$ curve data generation, and a dynamic loop condition (beating ventricle). Mock loop testing results will be compared to previously completed CFD simulations. This empirical data will be valuable in improving the CFD modeling and the Pediatric VAD design.

\subsubsection{Large Animal Model Studies}

Following the successful completion of the mock loop study, VAD V3 will be tested in two acute large ovine animal studies $(\sim 20 \mathrm{~kg})$. The animals will be cannulated to allow connection of the VAD prototype and the device will be 
evaluated to confirm stable hemodynamic support for up to 4 hours. The Inspired VAD will be evaluated for ease of use of the driver, pump insertion, degree of blood trauma (hemolysis levels must be $<10 \mathrm{mg} / \mathrm{dl}$ ), and evidence of thrombus at necropsy.

\subsubsection{Design Improvements}

The impeller and pump housing designs of the Inspired Pediatric VAD may benefit from several improvements based on lessons learned during the research described in this dissertation: A few key potential improvements include:

- Slow velocity areas were identified at the pump outlet at lower flows (below 1.00 L/min) (Figures E-5, E-6). Curving the outlet to be more of a radial discharge design, where the cutwater and outlet follow the curvature of the volute before gradually turning perpendicularly outward may help alleviate some of the low flow velocity areas.

- Currently the pump outflow is attached to the lower pump housing and the pump inflow is attached to the upper housing. Additionally, these ports are perpendicular to each other, meaning cannula tubing exits the pump in two different orientations. For ease of pump assembly and to improve pump ergonomics and usability, the pump ports could be 
molded into the upper pump housing and exit the pump parallel to each other.

- A shroud was added to the top of the impeller blades to improve impeller efficiency and reduce flow recirculation between blades. A negative to this feature was excess reverse flow leakage above the shroud that entered into the pump inflow stream and caused a large amount of flow pre-rotation at lower flow rates (Figure E-9). A possible improvement may be to remove a portion of the shroud at the entrance of the pump blades, creating a "partially open" impeller design. This change may result in improved flow dynamics at the impeller eye and would still provide a shroud as flow progresses through the blade passages, mitigating any recirculation between blades.

- An improvement that may increase impeller efficiency is to add small diffuser blades in the middle of the impeller blade passages. These diffusers should be about half the length of the impeller blades, follow the same arc length as the blades, and thinner. A previous study explored the use of these secondary diffuser blades in the design of a centrifugal blood pump and found they had a positive effect on efficiency and hemolysis [29]. 
- The formation of Taylor vortices in the radial blood gap surrounding the pump rotor body was an unexpected outcome in the analysis of VAD V3. These vortices appear to increase flow time around the impeller and create concentrated areas of higher shear. Several improvements may be implemented to help reduce the formation of the vortices. As described in previous studies, the addition of steps or corrugations on the rotor body surface may help reduce, or at least, control the formation of vortices. This improvement may also help to reduce the number of vortices that are formed and control the locations in which they do form. Another solution may be the addition of a secondary impeller at the base of the rotor body. This impeller may help to increase flow around the rotor body, drawing fluid from the pump volute down along the sides and back up through the center opening. It may also help to distribute the pressure more evenly between the pump volute and rotor body center opening. This design improvement is likely to cause a large change to the overall fluid dynamics within the pump, but may be investigated with future simulations.

\subsubsection{Parametric Analysis}

With the required geometry defined for VAD V3 (with the integration of the MagLev motor), optimization of several components of the design may now be performed. Utilizing a series of CFD simulations, parametric analysis looking at 
how overall pump efficiency is affected by design elements, such as blade discharge angle, blade thickness, radial and axial gaps may be performed.

\subsubsection{Additional Pump Designs}

Although the development of the Inspired Pediatric VAD is not completed (we have not achieved a design freeze), lessons learned from this dissertation research may be applied to the design and development of future single-use pumps to be used with the Inspired Universal MagLev System. Development of a pediatric pump/oxygenator hybrid for cardiopulmonary support is planned, with adult specific pump designs to follow. 


\section{REFERENCES}

[1] A. P. Ambrosy et al., "The global health and economic burden of hospitalizations for heart failure: Lessons learned from hospitalized heart failure registries," J. Am. Coll. Cardiol., vol. 63, no. 12, pp. 1123-1133, 2014.

[2] P. Ponikowski et al., "Heart failure: preventing disease and death worldwide," ESC Hear. Fail., vol. 1, no. 1, pp. 4-25, 2014.

[3] E. J. Benjamin et al., "Heart Disease and Stroke Statistics'2017 Update: A Report from the American Heart Association," Circulation, vol. 135, no. 10, pp. e146-e603, 2017.

[4] P. A. Heidenreich et al., "Forecasting the impact of heart failure in the united states a policy statement from the american heart association," Circ. Hear. Fail., vol. 6, no. 3, pp. 606-619, 2013.

[5] C. Cook, G. Cole, P. Asaria, R. Jabbour, and D. P. Francis, "The annual global economic burden of heart failure," Int. J. Cardiol., vol. 171, no. 3, pp. 368-376, 2014.

[6] M. Colvin et al., "OPTN/SRTR 2018 Annual Data Report: Heart," Am. J. Transplant., vol. 20, no. s1, pp. 340-426, 2020.

[7] "National Data - Organ Procurement and Transplantation Network, OPTN, U.S. Department of Health \& Human Services," 2020. [Online]. Available: https://optn.transplant.hrsa.gov/data/view-data-reports/national-data/. [Accessed: 16-Nov-2020].

[8] I. Adachi and R. D. B. Jaquiss, "Mechanical Circulatory Support in Children," Curr. Cardiol. Rev., vol. 12, no. 2, pp. 132-140, 2016.

[9] S. Burki and I. Adachi, "Pediatric ventricular assist devices: Current challenges and future prospects," Vasc. Health Risk Manag., vol. 13, pp. 177-185, 2017.

[10] J. W. Rossano et al., "Prevalence, morbidity, and mortality of heart failurerelated hospitalizations in children in the United States: A population-based study," J. Card. Fail., vol. 18, no. 6, pp. 459-470, 2012.

[11] C. S. D. Almond et al., "Waiting list mortality among children listed for heart 
transplantation in the United States," Circulation, vol. 119, no. 5, pp. 717727, 2009.

[12] K. M. Burns, "Paediatric heart failure research: Role of the National Heart, Lung, and Blood Institute," Cardiol. Young, vol. 25, no. S2, pp. 167-171, 2015.

[13] J. T. Baldwin et al., "Closing in on the PumpKIN Trial of the Jarvik 2015 Ventricular Assist Device," Semin. Thorac. Cardiovasc. Surg. Pediatr. Card. Surg. Annu., vol. 20, pp. 9-15, 2017.

[14] Y. R. Shin, Y. H. Park, and H. K. Park, "Pediatric ventricular assist device," Korean Circ. J., vol. 49, no. 8, pp. 678-690, 2019.

[15] B. Stiller, I. Adachi, and C. D. J. Fraser, "Pediatric ventricular assist devices.," Pediatr. Crit. care Med. a J. Soc. Crit. Care Med. World Fed. Pediatr. Intensive Crit. Care Soc., vol. 14, no. 5 Suppl 1, pp. S20-6, Jun. 2013.

[16] J. Baras Shreibati, J. D. Goldhaber-Fiebert, D. Banerjee, D. K. Owens, and M. A. Hlatky, "Cost-Effectiveness of Left Ventricular Assist Devices in Ambulatory Patients With Advanced Heart Failure," JACC Hear. Fail., vol. 5, no. 2, pp. 110-119, 2017.

[17] R. Schöb, "Centrifugal pump without bearings or seals," World Pumps, vol. 2002, no. 430, pp. 34-37, 2002.

[18] N. Mendler, F. Podechtl, G. Feil, P. Hiltmann, and F. Sebening, "Seal-less Centrifugal Blood Pump with Magnetically Suspended Rotor: Rot-a-Flot," Artif. Organs, vol. 19, no. 7, pp. 620-624, 1995.

[19] T. Siess and H. Reul, "Basic Design Criteria for Rotary Blood Pumps," in Rotary Blood Pumps, 2000, pp. 69-83.

[20] P. A. Smith, Y. Wang, S. Groß-Hardt, and R. Graefe, "Hydraulic design," Mech. Circ. Respir. Support, pp. 301-334, Jan. 2018.

[21] T. Masuzawa, M. Osa, and M. Mapley, "Motor design and impeller suspension," Mech. Circ. Respir. Support, pp. 335-377, Jan. 2018.

[22] L. H. Tompkins et al., "Design and Initial Computational Evaluation of a Pediatric MagLev Rotary Blood Pump," ASAIO J., 2020.

[23] O. Cordier, "Ähnlichkeitsbedingungen für Strömungsmaschinen," BWK Bd, vol. 6, no. 10, 1953.

[24] A. J. Stepanoff, Centrifugal and Axial Flow Pumps: Theory, Design, and Application. John Wiley and Sons Inc., 1957. 
[25] H. Petermann and C. Pfleiderer, Strömungsmaschinen. Springer Berlin Heidelberg, 2013.

[26] S. L. Dixon and C. Hall, Fluid Mechanics and Thermodynamics of Turbomachinery. Elsevier Science, 2010.

[27] P. Epple, F. Durst, and A. Delgado, "A theoretical derivation of the Cordier diagram for turbomachines," Proc. Inst. Mech. Eng. Part C J. Mech. Eng. Sci., vol. 225, no. 2, pp. 354-368, 2011.

[28] J. Tuzson, Centrifugal pump design. Milton Keynes UK : John Wiley \& Sons, 2000.

[29] V. Kannojiya, A. K. Das, and P. K. Das, "Numerical simulation of centrifugal and hemodynamically levitated LVAD for performance improvement," Artif. Organs, vol. 44, no. 2, pp. E1-E19, 2020.

[30] S. Mozafari, M. A. Rezaienia, G. M. Paul, M. T. Rothman, P. Wen, and T. Korakianitis, "The Effect of Geometry on the Efficiency and Hemolysis of Centrifugal Implantable Blood Pumps," ASAIO J., vol. 63, no. 1, pp. 53-59, 2017.

[31] J. F. Gülich, Centrifugal Pumps, 2nd ed. Springer-Verlag Berlin Heidelberg, 2010.

[32] K. X. Qian, P. Zeng, W. M. Ru, and H. Y. Yuan, "Stream lined design of im peller and its effect on pum p haem olysis," vol. 26, no. 2, pp. 79-81, 2002.

[33] S. D. Kyparissis, E. C. Douvi, E. E. Panagiotopoulos, and D. P. Margaris, "Parametric Study Performance of a Centrifugal Pump Based on Simple and Double-Arc Blade Design Methods," 3rd Int. Conf. Exp. Model. Optim., no. February 2016, pp. 8-11, 2009.

[34] I. J. Karassik, J. P. Messina, P. Cooper, and C. T. A.-T. T.- Heald, "Pump Handbook (4th Edition)." McGraw-Hill Professional Publishing, Blacklick, USA, 2007.

[35] A. R. Curtas, H. G. Wood, P. E. Allaire, J. C. McDaniel, S. W. Day, and D. B. Olsen, "Computational fluid dynamics modeling of impeller designs for the heartquest left ventricular assist device," ASAIO J., vol. 48, no. 5, pp. 552-561, 2002.

[36] A. J. Stepanoff, Centrifugal and axial flow Pumps, vol. 264, no. 5. John Wiley and Sons Inc., 1957.

[37] T. Shigemitsu, J. Fukutomi, and K. Kaji, "Influence of Blade Outlet Angle and Blade Thickness on Performance and Internal Flow Conditions of Mini Centrifugal Pump," Int. J. Fluid Mach. Syst., vol. 4, no. 3, pp. 317-323, 
2011.

[38] W. G. Kim et al., "Development of a centrifugal pump with thick blades," Artif. Organs, vol. 24, no. 2, pp. 160-164, 2000.

[39] V. S. Lobanoff and R. R. Ross, Centrifugal Pumps: Design and Application: Design and Application (Google eBook). Elsevier, 1992.

[40] J. B. Anderson, H. G. Wood, P. E. Allaire, G. Bearnson, and P. Khanwilkar, "Computational flow study of the continuous flow ventricular assist device, Prototype Number 3 blood pump," Artif. Organs, vol. 24, no. 5, pp. 377385, 2000.

[41] J. Wu, B. E. Paden, H. S. Borovetz, and J. F. Antaki, "Computational fluid dynamics analysis of blade tip clearances on hemodynamic performance and blood damage in a centrifugal ventricular assist device," Artif. Organs, vol. 34, no. 5, pp. 402-411, 2010.

[42] M. Nishida, T. Yamane, and B. Asztalos, "Washout hole flow measurement for the development of a centrifugal blood pump," Artif. Organs, vol. 22, no. 5, pp. 386-392, 1998.

[43] T. M. Lee et al., "Cardiomyopathy Compendium Pediatric Cardiomyopathies," pp. 855-873, 2017.

[44] A. Cheng, C. A. Williamitis, and M. S. Slaughter, "Comparison of continuous-flow and pulsatile-flow left ventricular assist devices: is there an advantage to pulsatility?," Ann. Cardiothorac. Surg., vol. 3, no. 6, pp. 57357381, 2014.

[45] E. D. Blume et al., "Outcomes of children implanted with ventricular assist devices in the United States: First analysis of the Pediatric Interagency Registry for Mechanical Circulatory Support (PediMACS)," J. Hear. Lung Transplant., vol. 35, no. 5, pp. 578-584, 2016.

[46] I. Adachi and C. D. Fraser, "Mechanical Circulatory Support for Infants and Small Children," Semin. Thorac. Cardiovasc. Surg. Pediatr. Card. Surg. Annu., vol. 14, no. 1, pp. 38-44, 2011.

[47] R. Hetzer et al., "Mechanical Cardiac Support in the Young With the Berlin Heart EXCOR Pulsatile Ventricular Assist Device: 15 Years' Experience," Pediatr. Card. Surg. Annu., vol. 9, no. 1, pp. 99-108, 2006.

[48] S. Lord et al., "Comparison of paracorporeal and continuous flow ventricular assist devices in children: Preliminary results," Eur. J. Cardiothoracic Surg., vol. 51, no. 4, pp. 709-714, 2017.

[49] M. Hosseinipour, R. Gupta, M. Bonnell, and M. Elahinia, "Rotary 
mechanical circulatory support systems," J. Rehabil. Assist. Technol. Eng., vol. 4, p. $205566831772599,2017$.

[50] S. Gross-Hardt et al., "Low-flow assessment of current ECMO/ECCO2R rotary blood pumps and the potential effect on hemocompatibility," Crit. Care, vol. 23, no. 1, pp. 1-9, 2019.

[51] C. Göbel et al., "Development of the MEDOS/HIA DeltaStream extracorporeal rotary blood pump," Artif. Organs, vol. 25, no. 5, pp. 358365, 2001.

[52] P. Adedayo, S. Wang, A. R. Kunselman, and A. Ündar, "Impact of Pulsatile Flow Settings on Hemodynamic Energy Levels Using the Novel Diagonal Medos DP3 Pump in a Simulated Pediatric Extracorporeal Life Support System," World J. Pediatr. Congenit. Hear. Surg., vol. 5, no. 3, pp. 440448, 2014.

[53] J. Zhang et al., "Optimization of a miniature Maglev ventricular assist device for pediatric circulatory support," ASAIO J., vol. 53, no. 1, pp. 2331, 2007.

[54] B. Falkner and S. R. Daniels, "Summary of the fourth report on the diagnosis, evaluation, and treatment of high blood pressure in children and adolescents," Hypertension, vol. 44, no. 4, pp. 387-388, 2004.

[55] C. L. Ogden et al., "Centers for Disease Control and Prevention 2000 growth charts for the United States: Improvements to the 1977 National Center for Health Statistics version," Pediatrics, vol. 109, no. 1, pp. 45-60, 2002.

[56] Centers for Disease Control and Prevention, "Growth Charts - Clinical Growth Charts," 2017. [Online]. Available:

https://www.cdc.gov/growthcharts/clinical_charts.htm. [Accessed: 23-Mar2020].

[57] B. Boville and L. C. Young, Quick guide to cardiopulmonary care. Irvine, CA: Edwards Lifesciences Corporation, 2015.

[58] G. N. Cattermole et al., "The normal ranges of cardiovascular parameters measured using the ultrasonic cardiac output monitor," Physiol. Rep., vol. 5, no. 6, pp. 1-9, 2017.

[59] C. Harvey, "Cannulation for neonatal and pediatric extracorporeal membrane oxygenation for cardiac support," Front. Pediatr., vol. 6, no. March, pp. 1-6, 2018.

[60] "Cannulae - Bio-Medicus NextGen | Medtronic." [Online]. Available: https://www.medtronic.com/us-en/healthcare- 
professionals/products/cardiovascular/cannulae/bio-medicus-nextgen.html. [Accessed: 16-Mar-2020].

[61] R. Eymard, T. Gallouët, and R. Herbin, "Finite volume methods," Handb. Numer. Anal., vol. 7, no. December, pp. 713-1018, 2000.

[62] A. Sobachkin and G. Dumnov, "Numerical Basis of CAD-Embedded CFD," in NAFEMS World Congress 2013, 2013, no. February, pp. 1-20.

[63] "SOLIDWORKS Flow Simulation: Sliding Mesh." [Online]. Available: https://files.goengineer.com/docs/support/SOLIDWORKS_Flow_Simulation _Sliding_Mesh.pdf. [Accessed: 16-Mar-2020].

[64] E. W. Errill, "Rheology of blood," Physiol. Rev., vol. 49, no. 4, pp. 863-888, 1969.

[65] D. Carswell et al., "Development of a radial ventricular assist device using numerical predictions and experimental haemolysis," Med. Eng. Phys., vol. 35, no. 8, pp. 1197-1203, 2013.

[66] K. Kido et al., "Computational Fluid Dynamics Analysis of the Pediatric Tiny Centrifugal Blood Pump (TinyPump)," Artif. Organs, vol. 30, no. 5, pp. 392399, May 2006.

[67] J. Zhang et al., "Computational and experimental evaluation of the fluid dynamics and hemocompatibility of the centrimag blood pump," Artif. Organs, vol. 30, no. 3, pp. 168-177, 2006.

[68] K. H. Fraser, T. Zhang, M. E. Taskin, B. P. Griffith, and Z. J. Wu, "A quantitative comparison of mechanical blood damage parameters in rotary ventricular assist devices: Shear stress, exposure time and hemolysis index," J. Biomech. Eng., vol. 134, no. 8, pp. 1-11, 2012.

[69] K. H. Fraser, M. E. Taskin, B. P. Griffith, and Z. J. Wu, "The use of computational fluid dynamics in the development of ventricular assist devices," Med. Eng. Phys., vol. 33, no. 3, pp. 263-280, 2011.

[70] F. M. White, Fluid mechanics. McGraw-hill, 1999.

[71] Y. Alemu and D. Bluestein, "Flow-induced platelet activation and damage accumulation in a mechanical heart valve: Numerical studies," Artif. Organs, vol. 31, no. 9, pp. 677-688, 2007.

[72] K. A. Dasse et al., "Assessment of hydraulic performance and biocompatibility of a MagLev centrifugal pump system designed for pediatric cardiac or cardiopulmonary support," ASAIO J., vol. 53, no. 6, pp. 771-777, 2007. 
[73] V. V. Yarlagadda et al., "Temporary Circulatory Support in U.S. Children Awaiting Heart Transplantation," J. Am. Coll. Cardiol., vol. 70, no. 18, pp. 2250-2260, 2017.

[74] I. Wilmot, A. Lorts, and D. Morales, "Pediatric Mechanical Circulatory Support," Korean J. Thorac. Cardiovasc. Surg., vol. 46, no. 6, pp. 391-401, Dec. 2013.

[75] T. Masuzawa, M. Osa, and M. Mapley, "Mechanical Circulatory and Respiratory Support - Motor design and impeller suspension," 1st ed., S. D. Gregory, M. C. Stevens, and J. F. B. T.-M. C. and R. S. Fraser, Eds. Academic Press, 2018, pp. 335-377.

[76] R. Schöb, "Centrifugal pump without bearings or seals," World Pumps, vol. 2002, no. 430, pp. 34-37, 2002.

[77] J. A. Larose, D. Tamez, M. Ashenuga, and C. Reyes, "Design concepts and principle of operation of the heartware ventricular assist system," ASAIO J., vol. 56, no. 4, pp. 285-289, 2010.

[78] N. Moazami et al., "Axial and centrifugal continuous-flow rotary pumps: A translation from pump mechanics to clinical practice," J. Hear. Lung Transplant., vol. 32, no. 1, pp. 1-11, 2013.

[79] K. Bourque et al., "HeartMate III: Pump design for a centrifugal LVAD with a magnetically levitated rotor," ASAIO J., vol. 47, no. 4, pp. 401-405, 2001.

[80] N. Barletta, "Design of a Bearingless Blood Pump," in 3rd International Symposium on Magnetic Suspension Technology, 1995, 1995, pp. 265274.

[81] A. Hughes and B. Drury, Electric Motors and Drives. 2013.

[82] ELECTRO-CRAFT CORPORATION USA, DC Motors, Speed Controls, Servo Systems: An Engineering Handbook, 3rd ed. Pergamon Press, 1972.

[83] S. Boës, G. Ochsner, R. Amacher, A. Petrou, M. Meboldt, and M. Schmid Daners, "Control of the Fluid Viscosity in a Mock Circulation," Artif. Organs, vol. 42, no. 1, pp. 68-77, 2018.

[84] J. A. Long, A. Ündar, K. B. Manning, and S. Deutsch, "Viscoelasticity of pediatric blood and its implications for the testing of a pulsatile pediatric blood pump," ASAIO J., vol. 51, no. 5, pp. 563-566, 2005.

[85] N. Watanabe et al., "Simulation of the BP-80 blood pump," Artif. Organs, vol. 25, no. 9, pp. 733-739, 2001. 
[86] M. Behr, D. Arora, Y. Nosé, and T. Motomura, "Performance analysis of ventricular assist devices using finite element flow simulation," Int. J. Numer. Methods Fluids, vol. 46, no. 12, pp. 1201-1210, 2004.

[87] A. L. Throckmorton et al., "Design of a continous flow centrifugal pediatric ventricular assist device," Int. J. Artif. Organs, vol. 26, no. 11, pp. 10151031, 2003.

[88] G. W. Burgreen et al., "Computational fluid dynamics analysis of a maglev centrifugal left ventricular assist device," Artif Organs, vol. 28, no. 10, pp. 874-880, 2004.

[89] M. Behbahani et al., "A review of computational fluid dynamics analysis of blood pumps,” Eur. J. Appl. Math., vol. 20, no. 4, pp. 363-397, 2009.

[90] M. N. Islam, H. Gomer, and S. Sacks, "Comparison of dimensional accuracies of stereolithography and powder binder printing," Int. J. Adv. Manuf. Technol., vol. 88, no. 9-12, pp. 3077-3087, 2017.

[91] T. D. Dikova, D. A. Dzhendov, D. Ivanov, and K. Bliznakova, "Dimensional accuracy and surface roughness of polymeric dental bridges produced by different 3D printing processes," Arch. Mater. Sci. Eng., vol. 94, no. 2, pp. 65-75, 2018.

[92] M. Colvin et al., "OPTN/SRTR 2015 Annual Data Report: Heart," Am. J. Transplant., vol. 17, pp. 286-356, 2017.

[93] A. I. Dipchand et al., "The Registry of the International Society for Heart and Lung Transplantation: Eighteenth Official Pediatric Heart Transplantation Report - 2015; Focus Theme: Early Graft Failure," J. Hear. Lung Transplant., vol. 34, no. 10, pp. 1233-1243, 2015.

[94] L. Tonks, "Note on earnshaw's theorem," Electr. Eng., vol. 59, no. 3, pp. 118-119, 1940.

[95] M. A. Rezaienia, G. Paul, E. Avital, M. Rothman, and T. Korakianitis, "Computational parametric study of the axial and radial clearances in a centrifugal rotary blood pump," ASAIO J., vol. 64, no. 5, pp. 643-650, 2018.

[96] K. H. Fraser et al., "The effect of impeller position on CFD calculations of blood flow in magnetically levitated centrifugal blood pumps," in ASME 2010 Summer Bioengineering Conference, SBC 2010, 2010, no. PARTS A AND B, pp. 119-120.

[97] S. S. Shibeshi and W. E. Collins, "The Rheology of Blood Flow in a Branched Arterial System," Appl. Rheol., vol. 15, no. 6, pp. 398-405, 2005. 
[98] A. D. Caballero and S. Laín, "Numerical simulation of non-Newtonian blood flow dynamics in human thoracic aorta," Comput. Methods Biomech. Biomed. Engin., vol. 18, no. 11, pp. 1200-1216, 2015.

[99] X. Song, H. G. Wood, S. W. Day, and D. B. Olsen, "Studies of Turbulence Models in a Computational Fluid Dynamics Model of a Blood Pump," Artif. Organs, vol. 27, no. 10, pp. 935-937, 2003.

[100] S. Crow et al., "Acquired von Willebrand syndrome in continuous-flow ventricular assist device recipients," Ann. Thorac. Surg., vol. 90, no. 4, pp. 1263-1269, 2010.

[101] B. Thamsen et al., "Numerical Analysis of Blood Damage Potential of the HeartMate II and HeartWare HVAD Rotary Blood Pumps," Artif. Organs, vol. 39, no. 8, pp. 651-659, 2015.

[102] B. Thamsen et al., "A two-stage rotary blood pump design with potentially lower blood trauma: A computational study," Int. J. Artif. Organs, vol. 39, no. 4, pp. 178-183, 2016.

[103] L. Wiegmann et al., "Blood Pump Design Variations and Their Influence on Hydraulic Performance and Indicators of Hemocompatibility," Ann. Biomed. Eng., vol. 46, no. 3, pp. 417-428, 2018.

[104] D. S.-K. Ting, "Chapter 1 - Introducing Flow Turbulence," in Basics of Engineering Turbulence, D. S.-K. B. T.-B. of E. T. Ting, Ed. Academic Press, 2016, pp. 3-18.

[105] J. Frêne, D. Nicolas, B. Degueurce, D. Berthe, and M. B. T.-T. S. Godet, Eds., "Chapter 11 - Superlaminar Flow Regimes," in Hydrodynamic Lubrication, vol. 33, Elsevier, 1990, pp. 347-386.

[106] D. Reay, C. Ramshaw, and A. B. T.-P. I. Harvey, Eds., "Chapter 5 Reactors," in Isotopes in Organic Chemistry, Oxford: ButterworthHeinemann, 2008, pp. 103-186.

[107] M. Carlos Álvarez, W. Vicente, F. Solorio, E. Mancilla, M. Salinas, and V. R. Zenit, "A study of the Taylor-Couette flow with finned surface rotation," J. Appl. Fluid Mech., vol. 12, no. 5, pp. 1371-1382, 2019.

[108] J. H. Ng, R. K. Jaiman, and T. T. Lim, "Interaction dynamics of longitudinal corrugations in Taylor-Couette flows," Phys. Fluids, vol. 30, no. 9, 2018.

[109] V. T. Turitto and C. L. Hall, "Mechanical factors affecting hemostasis and thrombosis," Thromb. Res., vol. 92, no. 6 SUPPL. 2, pp. 25-31, 1998.

[110] D. M. Wootton and D. N. Ku, "Fluid mechanics of vascular systems, diseases, and thrombosis," Annu. Rev. Biomed. Eng., no. 1, pp. 299-329, 
1999.

[111] P. Hochareon, K. B. Manning, A. A. Fontaine, J. M. Tarbell, and S. Deutsch, "Correlation of in vivo clot deposition with the flow characteristics in the 50 cc Penn State Artificial Heart: A preliminary study," ASAIO J., vol. 50, no. 6, pp. 537-542, 2004.

[112] A. Garon and M. I. Farinas, "Fast three-dimensional numerical hemolysis approximation," Artif. Organs, vol. 28, no. 11, pp. 1016-1025, 2004.

[113] X. Song, A. L. Throckmorton, H. G. Wood, J. F. Antaki, and D. B. Olsen, "Computational Fluid Dynamics Prediction of Blood Damage in a Centrifugal Pump," Artif. Organs, vol. 27, no. 10, pp. 938-941, 2003.

[114] "Validation Examples, SolidWorks Flow Simulation 2019," Tech. Ref., 2019. 


\section{APPENDIX A: ADDITIONAL DESIGN EQUATIONS}

Equations A.1 - A.6. Solving for the Euler Velocity Triangle at the impeller blade discharge under ideal flow conditions.

$$
\begin{aligned}
& u_{2}=\frac{\pi D_{2} n}{60}=\frac{\pi \times 0.0274 \mathrm{~m} \times 4255 \mathrm{RPM}}{60}=6.10 \mathrm{~m} / \mathrm{sec} \\
& C_{m 2}=K_{m 2} \sqrt{2 g H}=0.138 \sqrt{2 \times 9.81 \mathrm{~m} / \mathrm{sec}^{2} \times 1.93 \text { meter head }}=0.850 \mathrm{~m} / \mathrm{sec} \\
& C_{u 2}=u_{2}-\frac{C_{m 2}}{\tan \beta_{2}}=6.10 \mathrm{~m} / \mathrm{sec}-\frac{0.850 \mathrm{~m} / \mathrm{sec}}{\tan 22.5^{\circ}}=4.050 \mathrm{~m} / \mathrm{sec}
\end{aligned}
$$

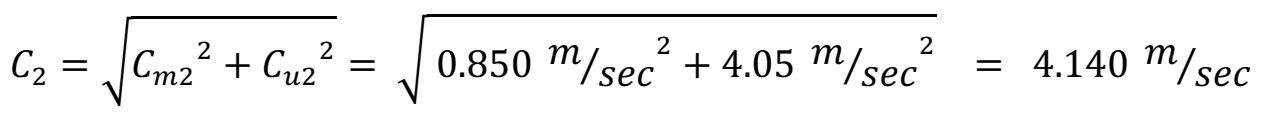

$$
\begin{aligned}
& \alpha_{2}=\tan ^{-1} \frac{c_{m 2}}{c_{u 2}}=\tan ^{-1} \frac{0.850 \mathrm{~m} / \mathrm{sec}}{4.050 \mathrm{~m} / \mathrm{sec}}=11.88^{\circ} \\
& b_{2}=\frac{Q}{\pi D_{2} C_{m 2}}=\frac{5.833 \times 10^{-5} \mathrm{~m}^{3} / \mathrm{sec}}{\pi \times 0.0274 \mathrm{~m} \times 0.850 \mathrm{~m} / \mathrm{sec}}=0.797 \mathrm{~mm} \rightarrow 1.00 \mathrm{~mm}
\end{aligned}
$$


Equations A.7 - A.12. Solving for the Euler Velocity Triangle at the impeller blade entrance under ideal flow conditions.

$$
\begin{gathered}
u_{1}=\frac{\pi D_{1} n}{60}=\frac{\pi \times 0.008 \mathrm{~m} \times 4255 \mathrm{RPM}}{60}=1.78 \mathrm{~m} / \mathrm{sec} \\
C_{m 1}=K_{m 1} \sqrt{2 g H}=0.195 \sqrt{2 \times 9.81 \mathrm{~m} / \mathrm{sec}^{2} \times 1.93 \mathrm{~meter} h e a d}=1.20 \mathrm{~m} / \mathrm{sec} \\
C_{u 1}=u_{1}-\frac{C_{m 1}}{\tan \beta_{1}}=1.78 \mathrm{~m} / \mathrm{sec}-\frac{1.20 \mathrm{~m} / \mathrm{sec}}{\tan 33.98^{\circ}}=0.002 \mathrm{~m} / \mathrm{sec} \\
C_{1}=\sqrt{C_{m 1}^{2}+C_{u 1}^{2}=\sqrt{1.20 \mathrm{~m} / \mathrm{sec}+0.002 \mathrm{~m} / \mathrm{sec}^{2}}=1.20 \mathrm{~m} / \mathrm{sec}} \\
\beta_{1}=\tan ^{-1} \frac{C_{m 1}}{u_{1}}=\tan ^{-1} \frac{1.20 \mathrm{~m} / \mathrm{sec}}{1.7 \mathrm{~m}^{2} / \mathrm{sec}}=33.98^{\circ} \\
\alpha_{1}=\tan ^{-1} \frac{c_{m 1}}{C_{u 1}}=\tan ^{-1} \frac{1.20 \mathrm{~m} / \mathrm{sec}}{0.002 \mathrm{~m} / \mathrm{sec}}=89.90^{\circ}
\end{gathered}
$$


Equations A.13 - A.16. Solving for the Euler Velocity Triangle at the impeller blade discharge while accounting for flow slip.

$$
\begin{aligned}
& C_{u 2}{ }^{\prime}=u_{2}\left(S-\phi \tan \beta_{2}\right)=6.10 \mathrm{~m} / \mathrm{sec}\left(0.563-.115 \tan 22.50^{\circ}\right)=3.14 \mathrm{~m} / \mathrm{sec}
\end{aligned}
$$

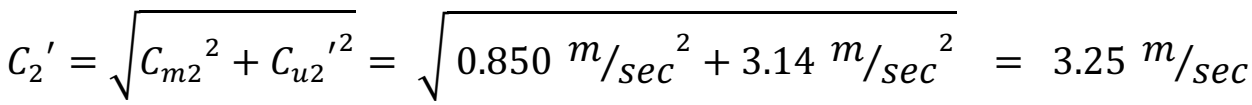

$$
\begin{aligned}
& \alpha_{2}{ }^{\prime}=\tan ^{-1} \frac{C_{m 2}}{C_{u 2}{ }^{\prime}}=\tan ^{-1} \frac{0.850 \mathrm{~m} / \mathrm{sec}}{3.14^{\mathrm{m} / \mathrm{sec}}}=15.15^{\circ} \\
& \beta_{2}^{\prime}=\tan ^{-1} \frac{C_{m 2}}{u_{2}-C_{u 2}{ }^{\prime}}=\tan ^{-1} \frac{0.850 \mathrm{~m} / \mathrm{sec}}{6.10^{\mathrm{m} / \mathrm{sec}}-3.14 \mathrm{~m} / \mathrm{sec}}=16.02^{\circ}
\end{aligned}
$$


Equations A.17 - A.20. Solving for the Euler Velocity Triangle at the impeller blade entrance while accounting for flow pre-rotation.

$$
\begin{gathered}
R_{1}=\frac{P_{1 s}}{C_{m 1}} \rightarrow P_{1 s}=R_{1} \times C_{m 1}=1.20 \times 1.20 \mathrm{~m} / \mathrm{sec}=1.44 \mathrm{~m} / \mathrm{sec} \\
C_{u 1}{ }^{\prime}=U_{1}-\frac{C_{m 1}}{\tan \beta_{1}{ }^{\prime}}=1.78 \mathrm{~m} / \mathrm{sec}-\frac{1.20 \mathrm{~m} / \mathrm{sec}}{\tan 40.00^{\circ}}=0.35 \mathrm{~m} / \mathrm{sec} \\
C_{1}{ }^{\prime}=\sqrt{{C_{m 1}}^{2}+{C_{u 1}}^{\prime 2}}=\sqrt{1.20 \mathrm{~m} / \mathrm{sec}^{2}+0.350^{2} / \mathrm{sec}^{2}}=1.25 \mathrm{~m} / \mathrm{sec} \\
\beta_{1}{ }^{\prime}=\tan ^{-1} \frac{P_{1 s}}{u_{1}}=\tan ^{-1} \frac{1.44 \mathrm{~m} / \mathrm{sec}}{1.7 \mathrm{~m}^{\mathrm{m} / \mathrm{sec}}}=39.05^{\circ} \rightarrow \mathbf{4 0 . 0 0}^{\circ}
\end{gathered}
$$




\section{APPENDIX B: CHAPTER 3 SUPPLEMENT}

Table B-1. Summary of Reynolds number calculations for pump inlet and impellers for Pumps V1 and V2 at $Q=3.5 \mathrm{~L} / \mathrm{min}$ and various rotational speeds ( $n$ ).

\begin{tabular}{|c|cccccc|}
\cline { 2 - 6 } \multicolumn{1}{c|}{} & \multicolumn{5}{c|}{ Pump Inlet - Flow through a Circular Pipe } \\
\cline { 2 - 7 } \multicolumn{1}{c|}{} & $\begin{array}{c}\text { Flow Rate, } \\
Q(\text { L/min })\end{array}$ & $\begin{array}{c}\text { Inlet Velocity, } \\
V(\mathrm{~m} / \mathrm{s})\end{array}$ & $\begin{array}{c}\text { Inlet } \\
\text { Diameter, } \\
D(\mathrm{~mm})\end{array}$ & Formula & $\operatorname{Re}$ & $\begin{array}{c}\text { Critical } \\
\text { Transition } \\
\operatorname{Re}\end{array}$ \\
\hline $\begin{array}{c}\text { Pump V1 } \\
\text { and V2 }\end{array}$ & 3.5 & 1.87 & 6.35 & $\operatorname{Re}=\frac{\rho V D}{\mu}$ & 3403 & 2300 \\
\hline
\end{tabular}

\begin{tabular}{|c|c|c|c|c|c|}
\hline & \multicolumn{5}{|c|}{ Impeller - Flow across a Rotating Disk } \\
\hline & $\begin{array}{c}\text { Rotational Speed, } \\
n \text { (RPM) }\end{array}$ & $\begin{array}{c}\text { Impeller } \\
\text { Radius, } r(\mathrm{~mm})\end{array}$ & Formula & $\operatorname{Re}$ & $\begin{array}{c}\text { Critical } \\
\text { Transition } \\
\text { Re }\end{array}$ \\
\hline \multirow{6}{*}{ Pump V1 } & 4255 & \multirow{5}{*}{13.7} & \multirow{5}{*}{$R e=\frac{2 r^{2} \rho n}{\mu}$} & 47933 & \multirow{5}{*}{$10^{6}$} \\
\hline & 4500 & & & 50693 & \\
\hline & 5000 & & & 56325 & \\
\hline & 5500 & & & 61958 & \\
\hline & 6000 & & & 67590 & \\
\hline & $\begin{array}{c}\text { Rotational Speed, } \\
n \text { (RPM) }\end{array}$ & $\begin{array}{c}\text { Impeller } \\
\text { Radius, } r(\mathrm{~mm})\end{array}$ & Formula & $\mathrm{Re}$ & $\begin{array}{c}\text { Critical } \\
\text { Transition } \\
\operatorname{Re}\end{array}$ \\
\hline \multirow{5}{*}{ Pump V2 } & 4255 & \multirow{5}{*}{16.1} & \multirow{5}{*}{$R e=\frac{2 r^{2} \rho n}{\mu}$} & 66198 & \multirow{5}{*}{$10^{6}$} \\
\hline & 4500 & & & 70009 & \\
\hline & 5000 & & & 77788 & \\
\hline & 5500 & & & 85567 & \\
\hline & 6000 & & & 93346 & \\
\hline
\end{tabular}


Table B-2. Four meshing schemes with increasing mesh refinement compared to respective number of mesh cells and converged values for $\triangle P$ and Torque for both Pump V1 and Pump V2 at $Q=3.5 \mathrm{~L} / \mathrm{min}$ and $n=$ 4255 RPM.

\begin{tabular}{|c|cccc|}
\cline { 2 - 5 } \multicolumn{1}{c|}{} & Mesh Scheme & Number of Cells & $\Delta \mathrm{P}(\mathrm{mmHg})$ & Torque $(\mathrm{N}-\mathrm{mm})$ \\
\hline \multirow{4}{*}{ Pump V1 } & 1 & 84,102 & 98.08 & 5.49 \\
& 2 & 188,412 & 97.20 & 5.62 \\
& 3 & 484,712 & 90.80 & 5.79 \\
& 4 & $1,143,915$ & 89.70 & 6.10 \\
\hline \hline \multirow{4}{*}{ Pump V2 } & 1 & 41,593 & 132.30 & 6.88 \\
& 2 & 123,504 & 123.40 & 7.19 \\
& 3 & 317,965 & 119.99 & 7.38 \\
& 4 & 690,285 & 119.50 & 7.40 \\
\hline
\end{tabular}

\section{B.1 CFD Simulation Time Step}

SolidWorks Technical Reference [62], [114] recommends setting a calculation time step of less than $1 / 50$ of the revolution period for transient (time dependent) simulations with rotating regions $\left(\Delta \mathrm{t}_{\mathrm{a}}\right)$. When dealing specifically with impellers, it recommends taking into account the absolute time required for one blade to move from its position to the position of the neighboring blade (our impeller has six blades) $\left(\Delta \mathrm{t}_{\mathrm{b}}\right)$. Taking these recommendations into consideration, we can calculate both time step versions as (using 1000 RPM as an example):

$$
\begin{gathered}
\frac{1000 \text { revolutions }}{1 \mathrm{~min}} \times \frac{1 \mathrm{~min}}{60 \mathrm{~seconds}}=16.67 \mathrm{revs} / \mathrm{sec} \rightarrow 0.060 \mathrm{sec} / \mathrm{rev} \\
\Delta t_{a}=\frac{0.060 \mathrm{sec} / \mathrm{rev}}{50}=0.0012 \mathrm{~seconds} \\
\Delta t_{b}=\frac{60 \text { seconds }}{1000 \text { RPM } \times 6 \text { Blades }}=0.01 \text { seconds }
\end{gathered}
$$


Time steps based on both of these methods were calculated for various impeller rotational speeds and are shown in Table B-3.

Table B-3. Calculated simulation time steps based on two recommended methods for various impeller rotational speeds.

\begin{tabular}{|ccc|}
\hline \multicolumn{3}{|c|}{ Calculated Simulation Time Steps } \\
\hline Speed (RPM) & $\Delta \mathrm{t}_{\mathrm{a}}(\mathrm{sec})$ & $\Delta \mathrm{t}_{\mathrm{b}}(\mathrm{sec})$ \\
\hline 1000 & 0.00120 & 0.0100 \\
2000 & 0.00060 & 0.0050 \\
3000 & 0.00040 & 0.0033 \\
4000 & 0.00030 & 0.0025 \\
5000 & 0.00024 & 0.0020 \\
6000 & 0.00020 & 0.0017 \\
\hline
\end{tabular}

In these CFD simulations, the automatic time step tool was used in SolidWorks with a threshold set at the finest calculated time step from Table B-3, 0.0002 seconds. On average, the automatic time steps in SolidWorks were much finer, in the range of $0.00005-0.00008$ seconds. 
Table B-4. Mean wall shear stress (WSS) values for all surfaces on the pump impeller and pump housing for both versions Pump V1 and V2 at rotational speeds 4255 to 6000 RPM.

\begin{tabular}{|ccc|}
\hline \multicolumn{3}{|c|}{ Impeller Surface Mean WSS (Pa) } \\
\hline Speed (RPM) & Pump V1 & Pump V2 \\
\hline 4255 & 73.56 & 67.32 \\
4500 & 78.50 & 71.55 \\
5000 & 88.86 & 81.00 \\
5500 & 98.91 & 91.93 \\
6000 & 110.51 & 101.84 \\
\hline \\
\multicolumn{3}{c}{ Pump Housing Surface Mean WSS (Pa) } \\
\hline Speed (RPM) & Pump V1 & Pump V2 \\
\hline 4255 & 54.43 & 70.83 \\
4500 & 55.78 & 80.30 \\
5000 & 65.61 & 88.65 \\
6500 & 74.70 & 103.68 \\
& 84.49 & 115.30 \\
\hline
\end{tabular}

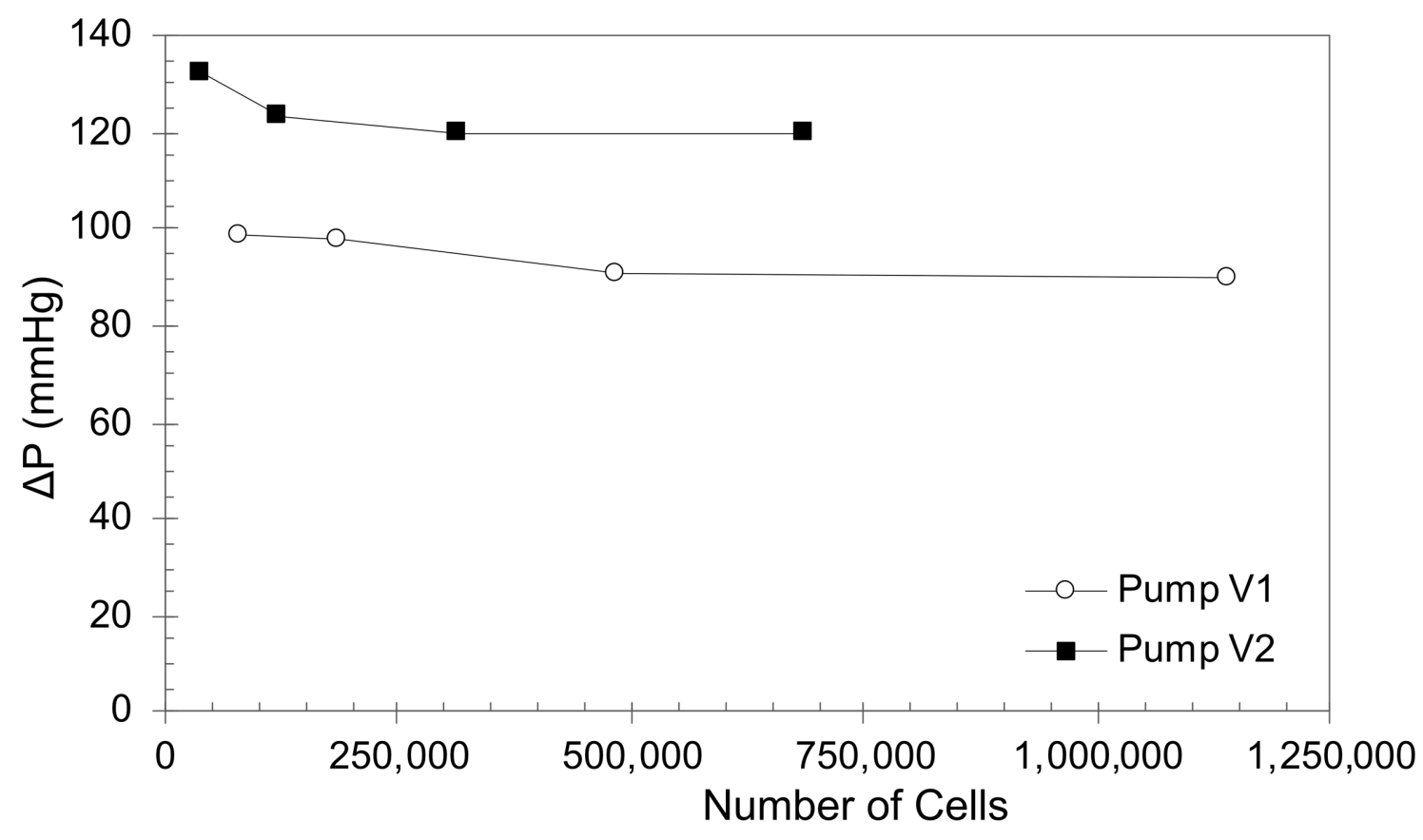

Figure B-1. Plotted converged $\triangle P$ values versus number of mesh cells for four meshing schemes for both Pump V1 and V2 at $Q=3.5 \mathrm{~L} / \mathrm{min}$ and $n$ $=4255$ RPM. 

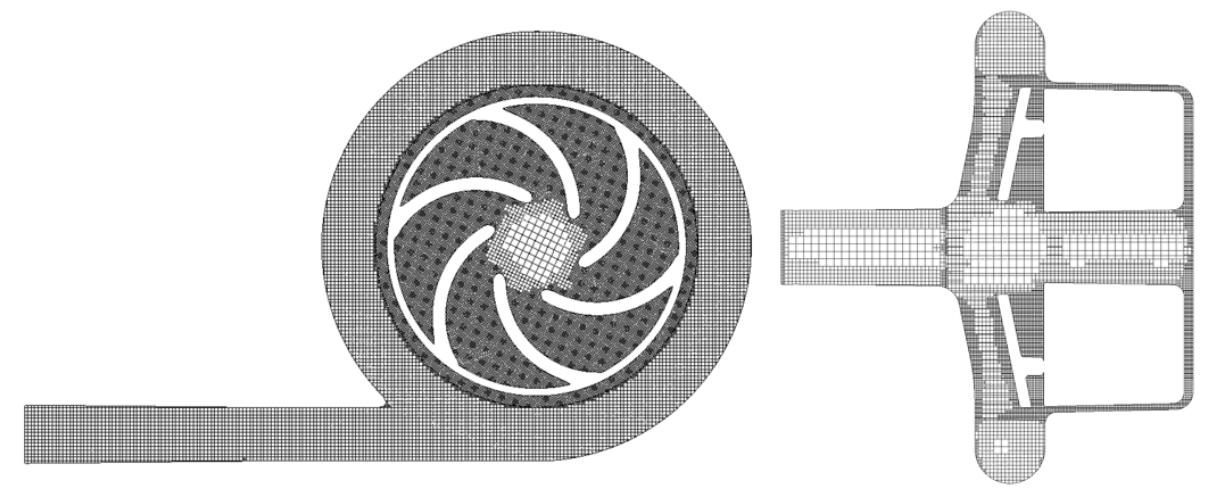

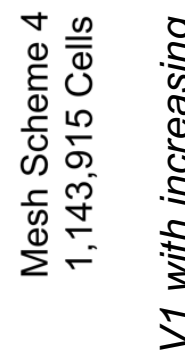
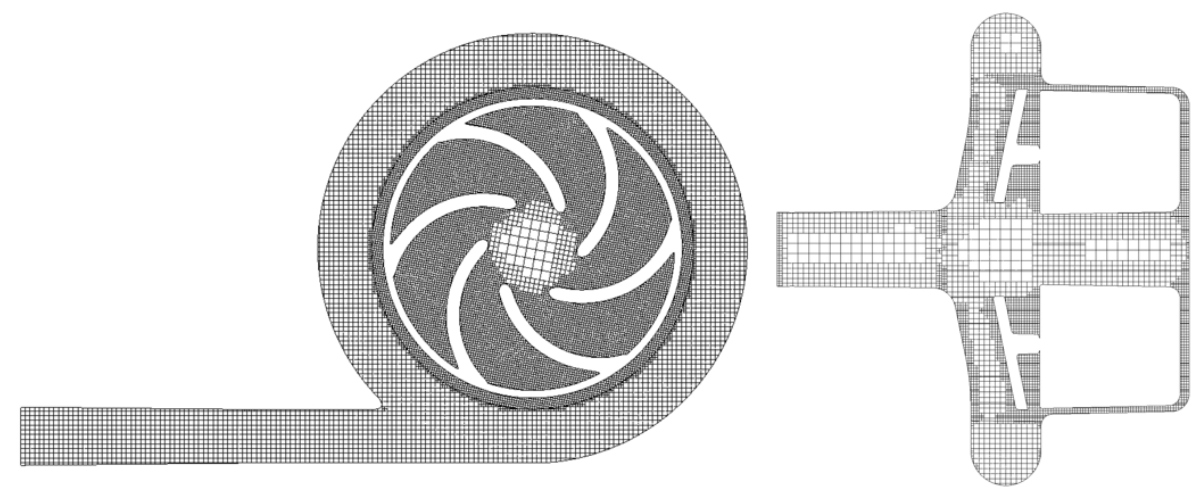

ह
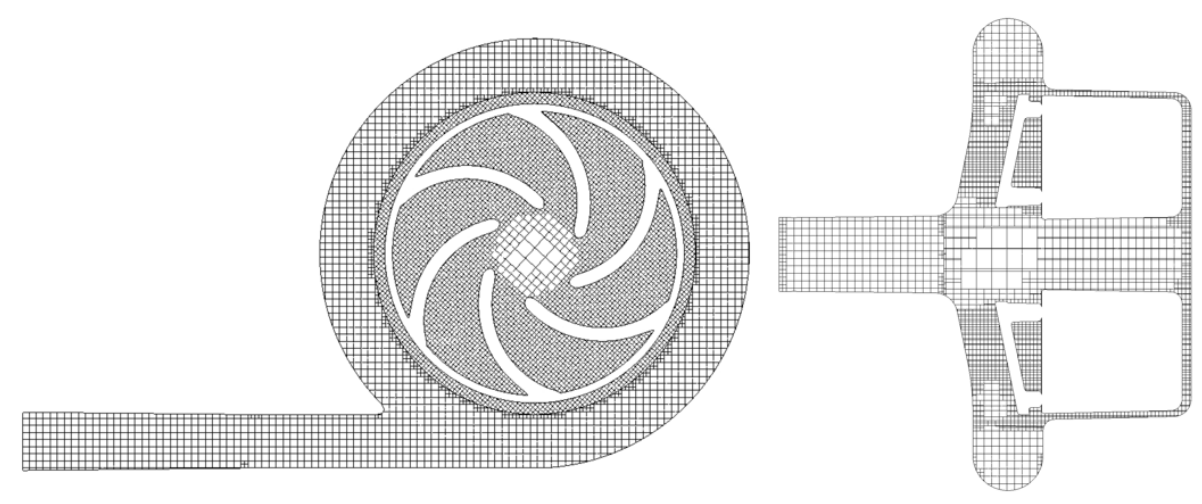

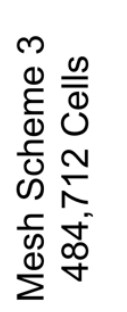

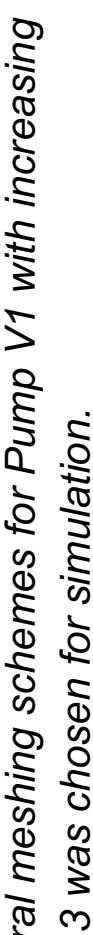

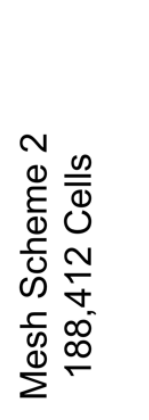

के
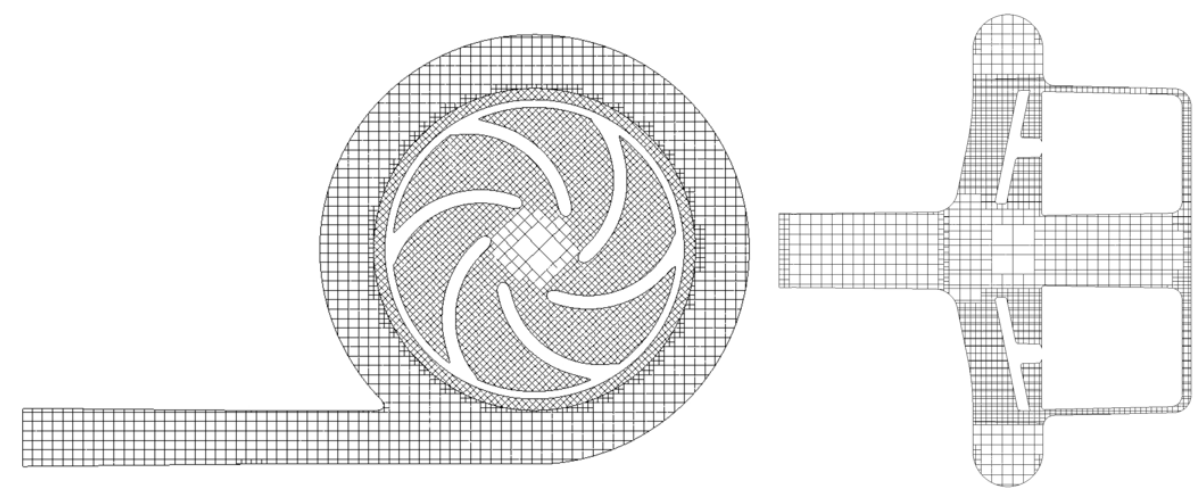

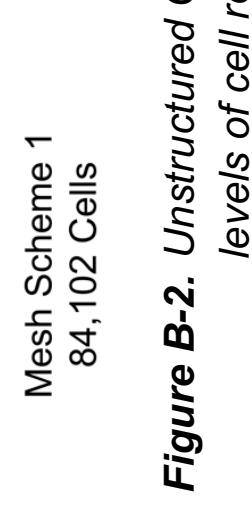




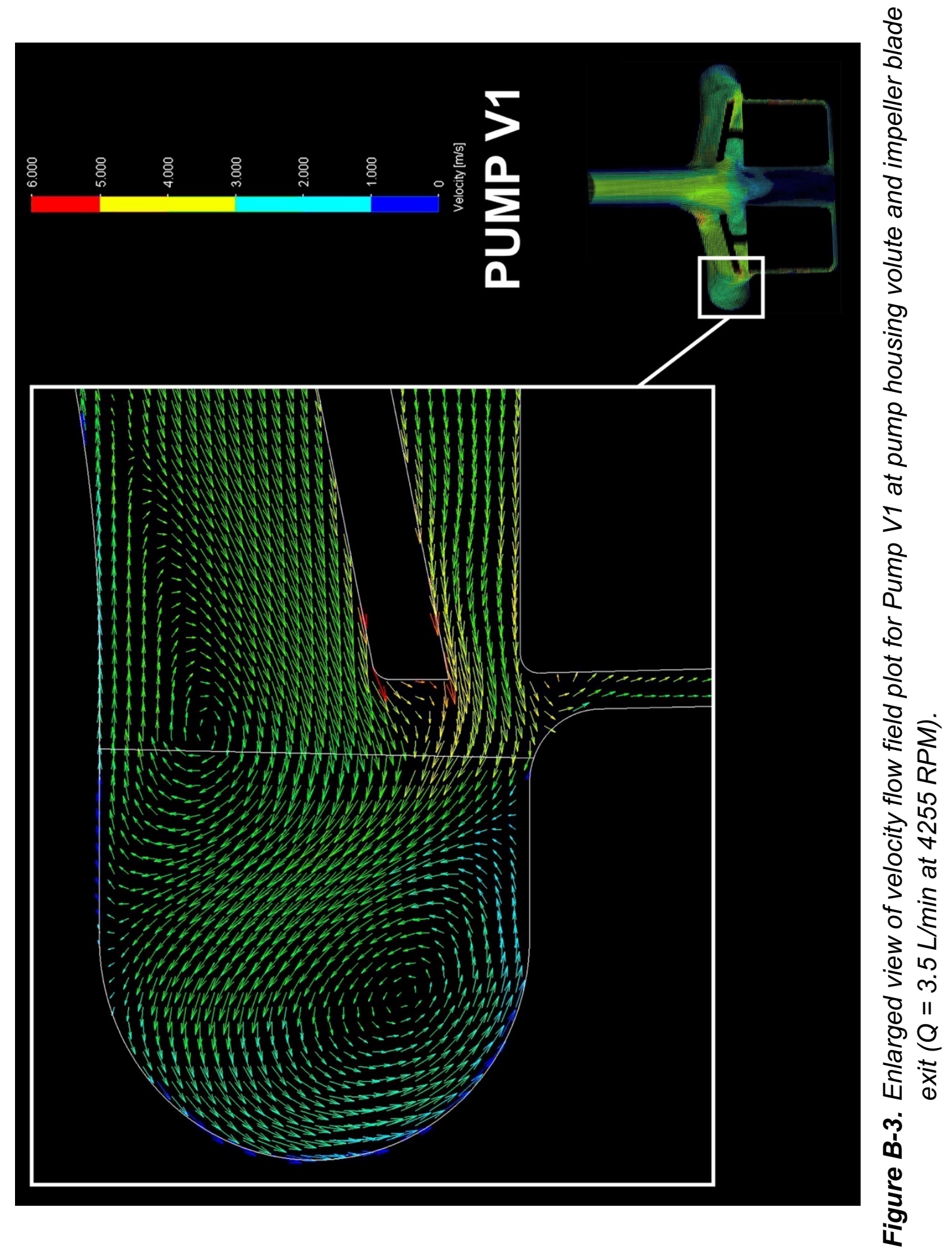




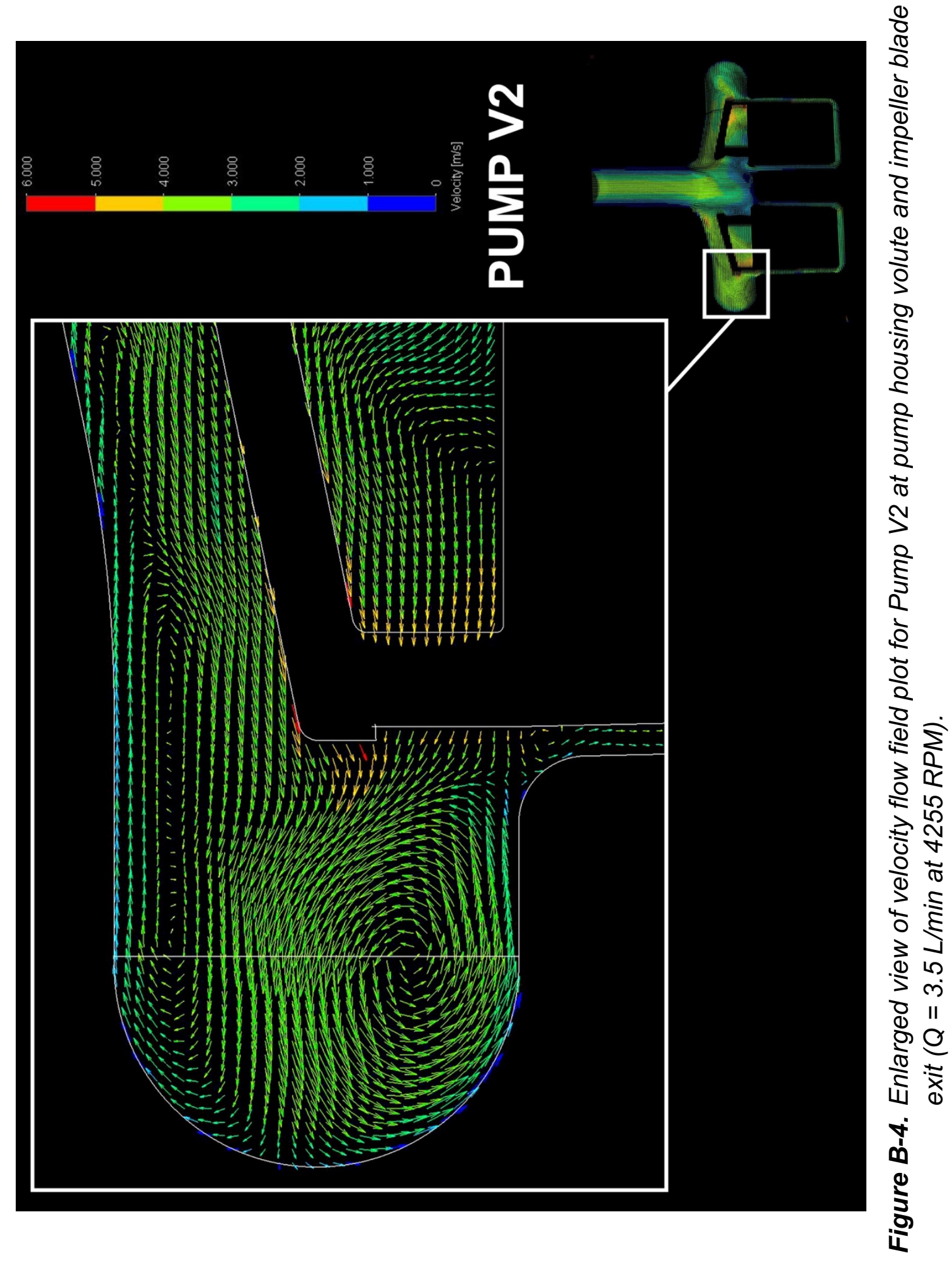




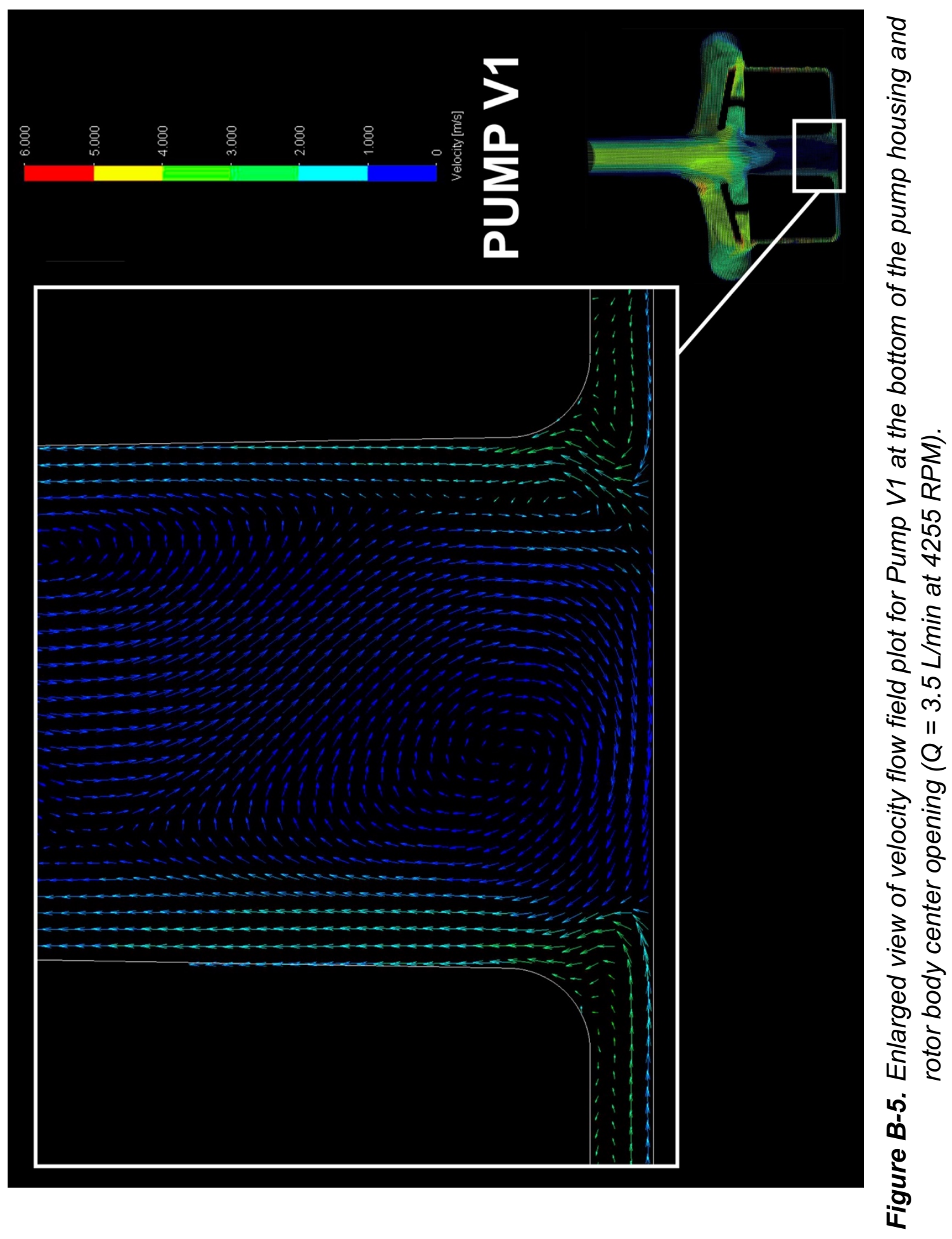



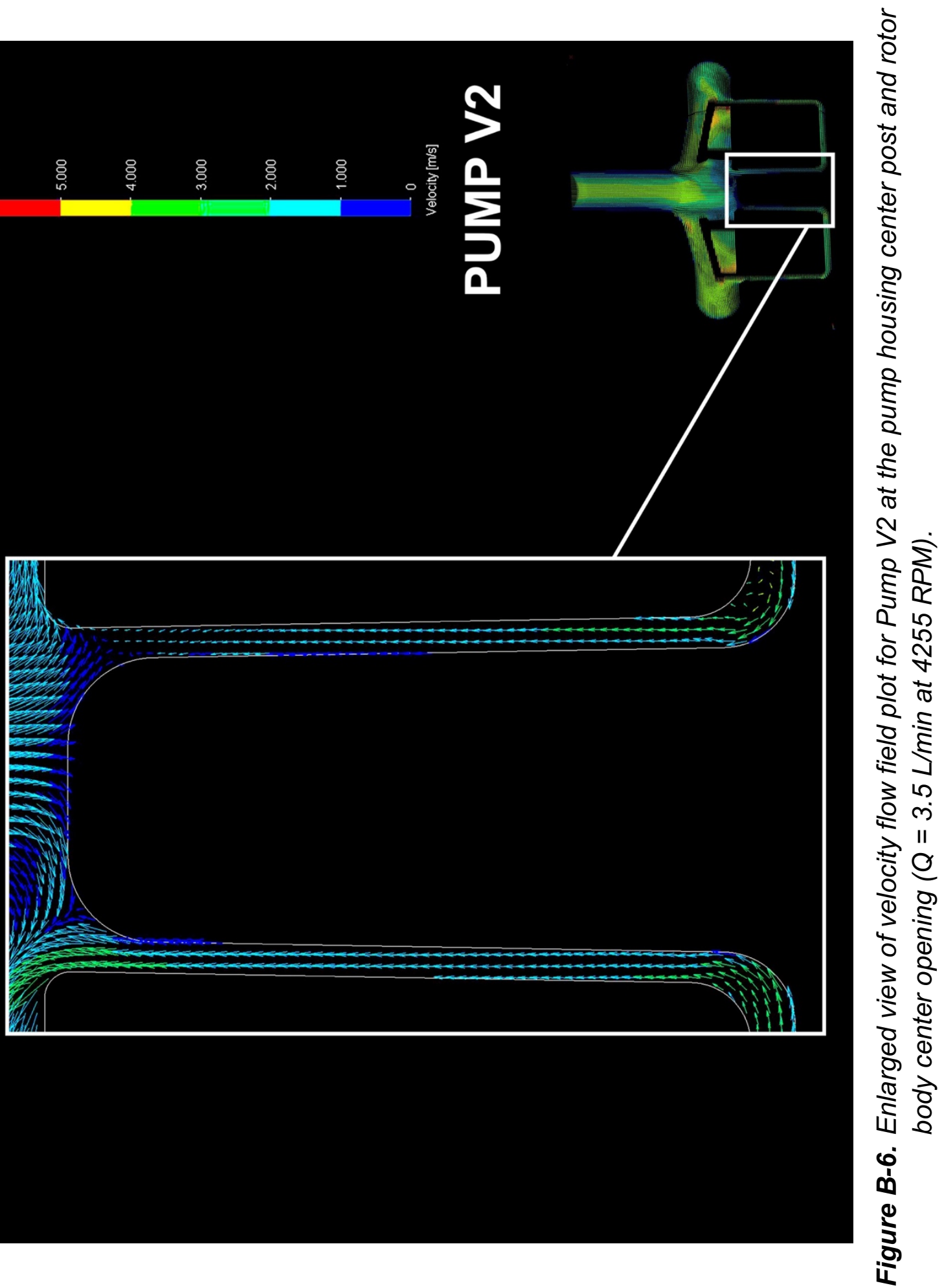


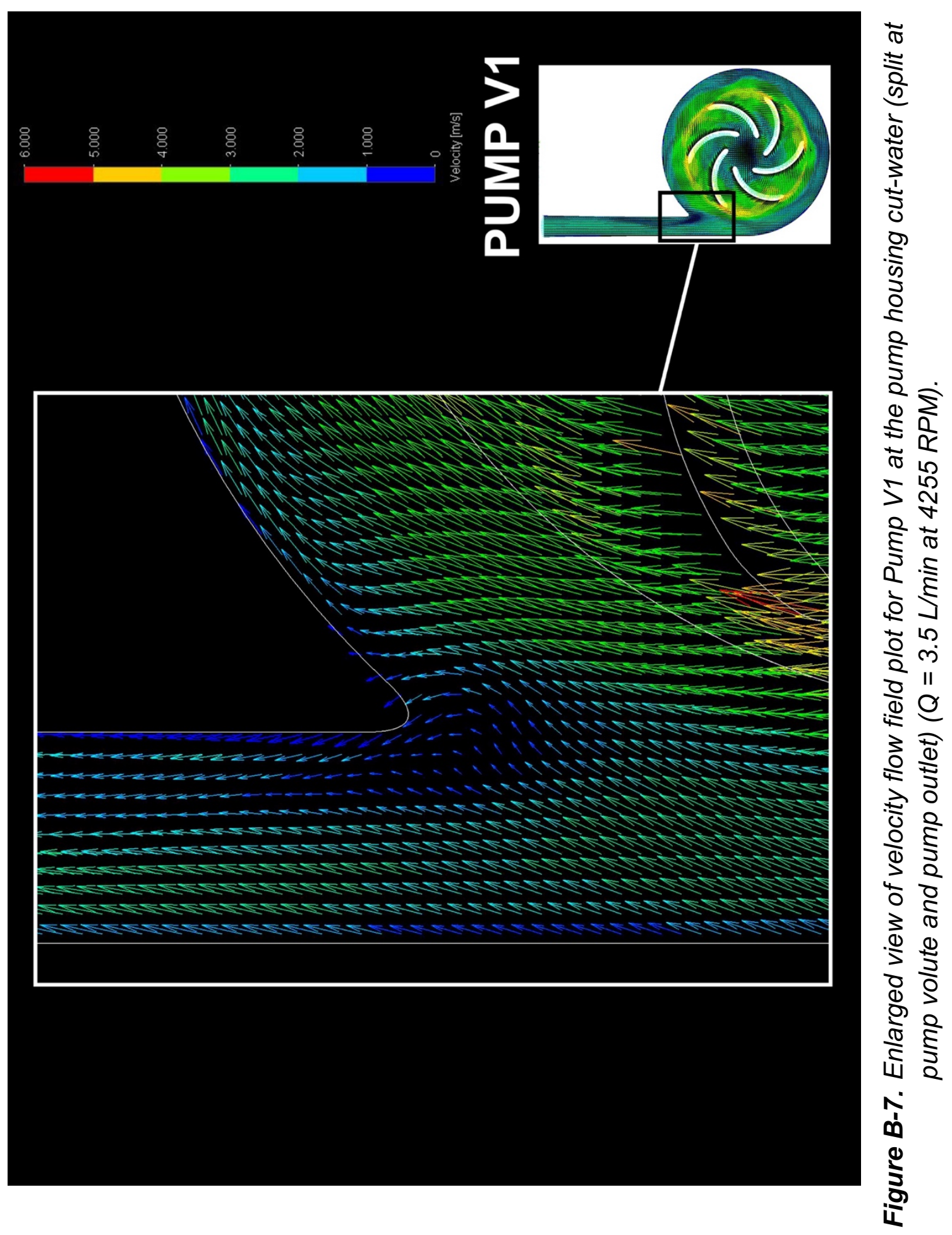




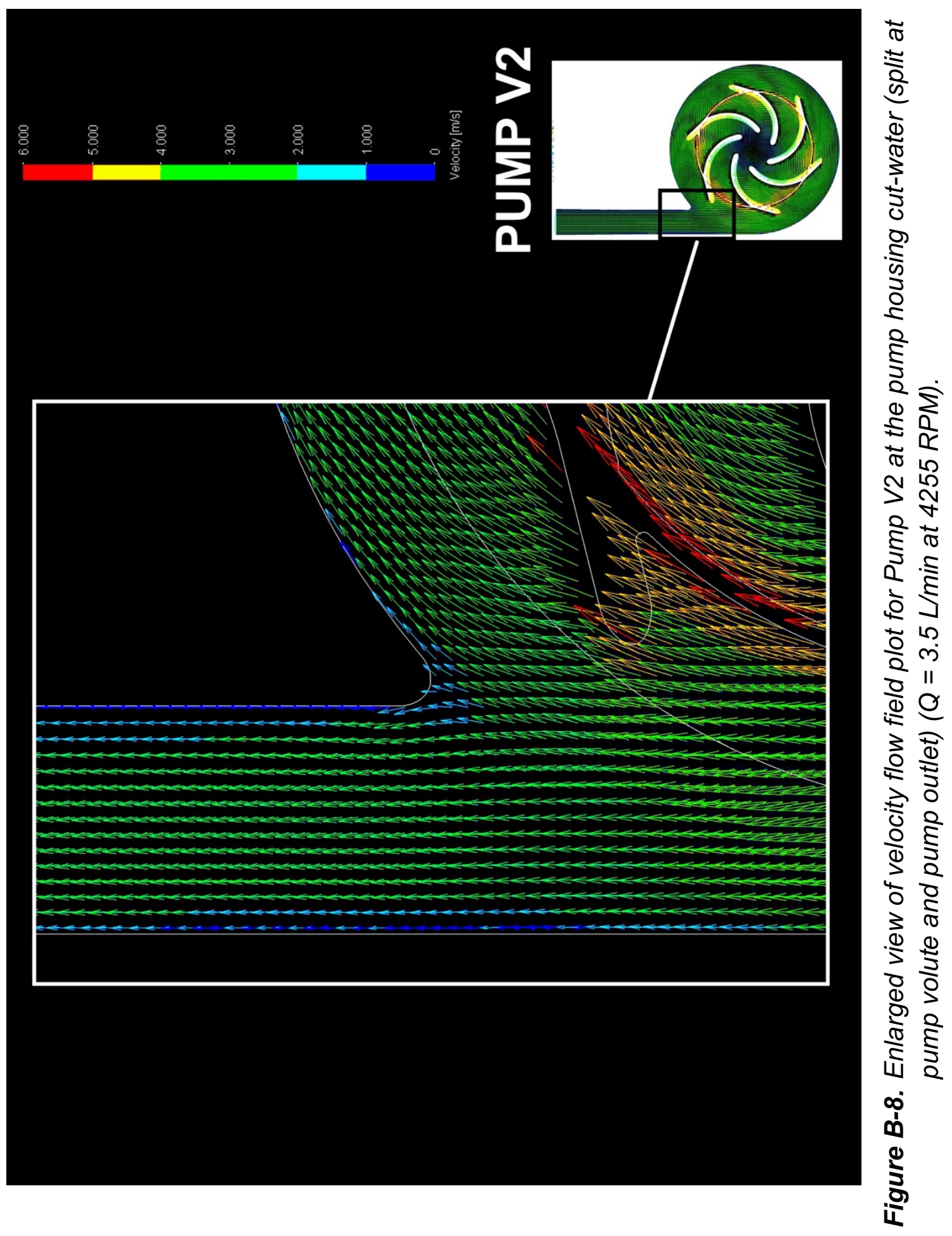




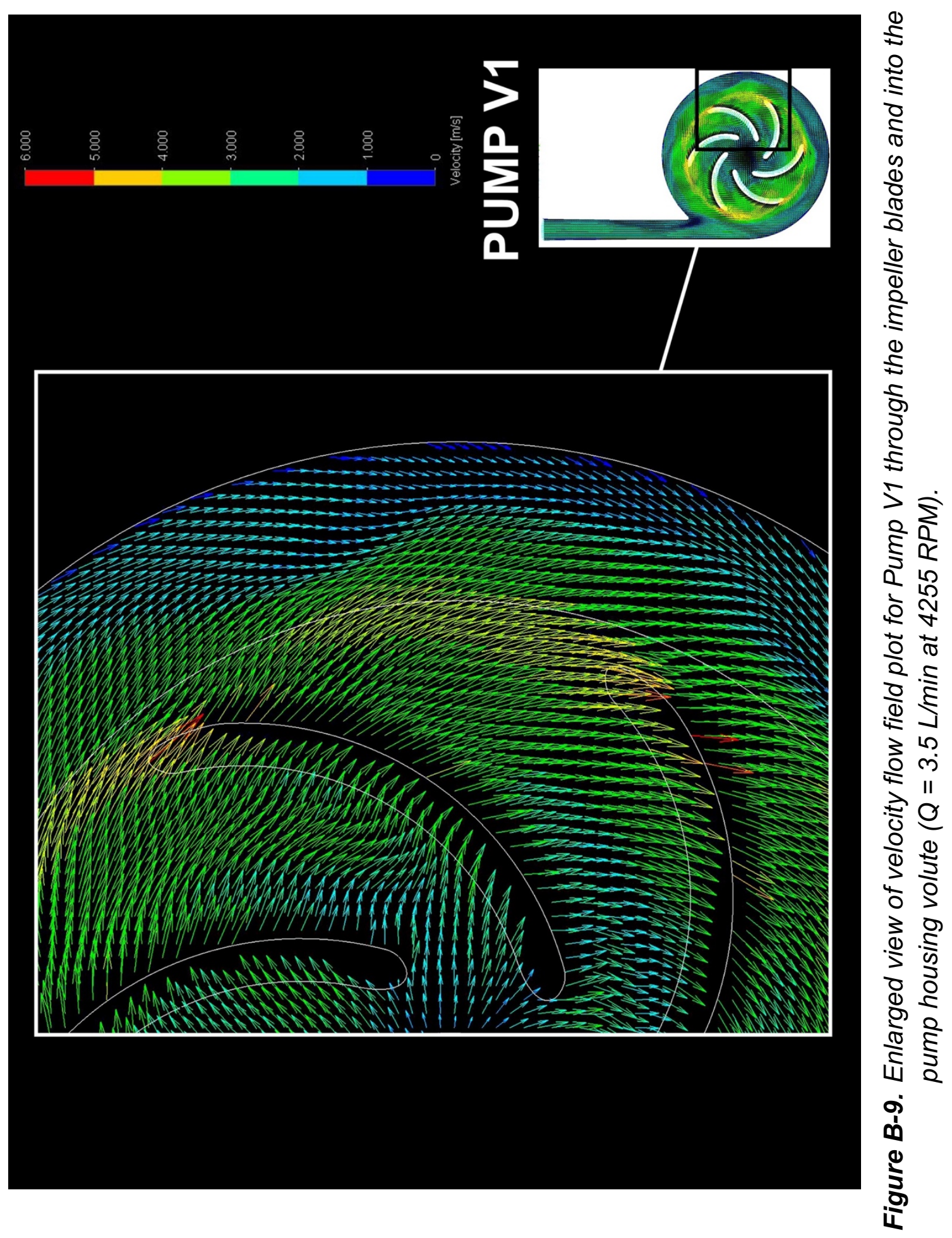




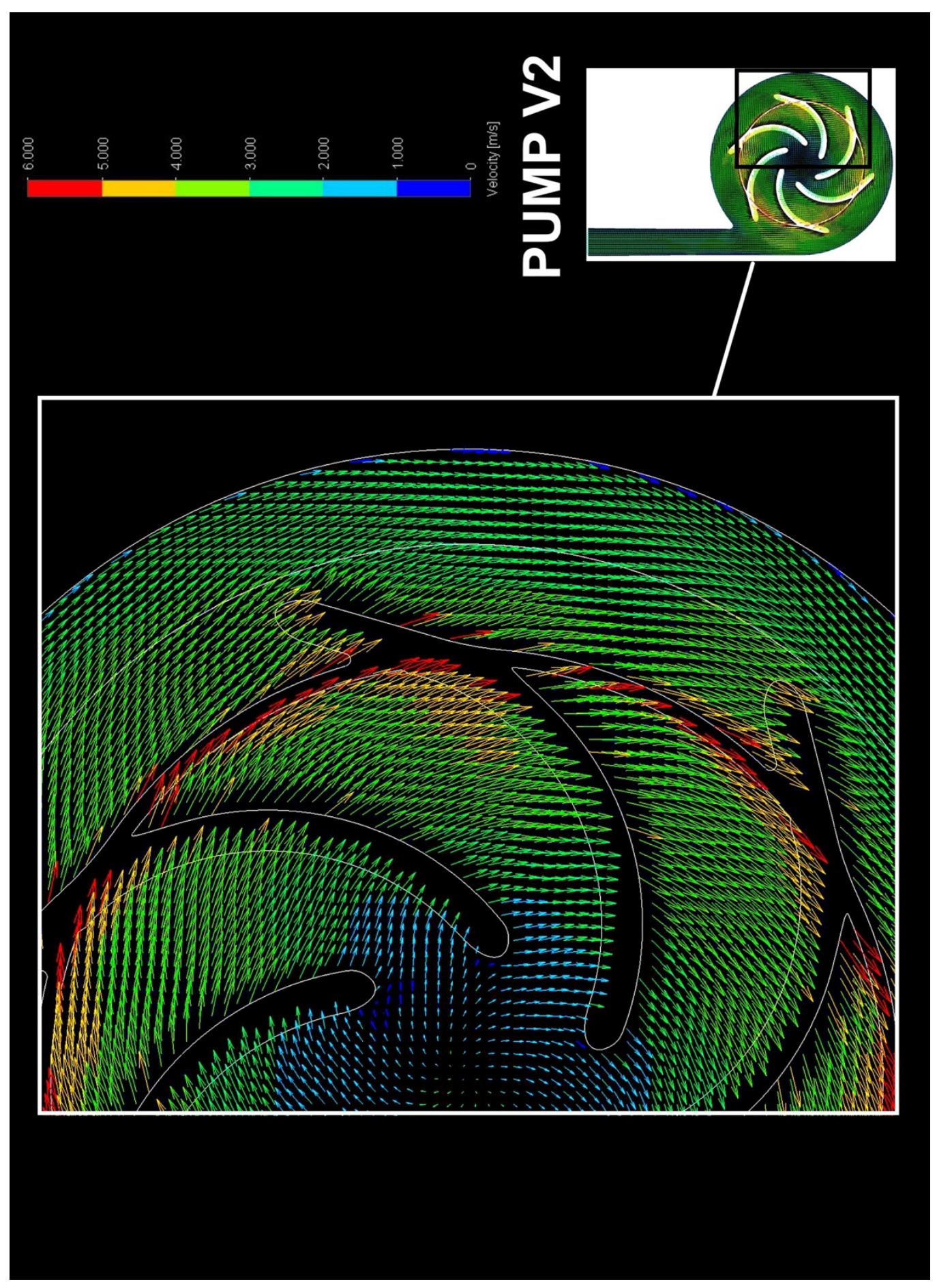




\section{APPENDIX C: CHAPTER 4 SUPPLEMENT}

Table C-1. DC motor back electromotive force (EMF) at corresponding rotational speeds, generated voltage, calculated back EMF constant, $K_{e}$, and motor torque constant, $K_{t}$.

\begin{tabular}{|ccc|}
\hline $\begin{array}{c}\text { Speed } \\
(R P M)\end{array}$ & $\begin{array}{c}\text { Voltage } \\
(V)\end{array}$ & $\begin{array}{c}\text { Back EMF, } K_{e} \\
(\text { V/rpm })\end{array}$ \\
\hline 400 & 1.34 & 0.00335 \\
800 & 2.68 & 0.00335 \\
1000 & 3.31 & 0.00331 \\
1500 & 4.92 & 0.00328 \\
7500 & 24.5 & 0.00327 \\
\hline \multicolumn{3}{c}{$K_{e}$} \\
$($ V/krpm) & & $K_{t}$ \\
& & 31.57 \\
\hline \multicolumn{2}{|c|}{3.311} & \\
\hline
\end{tabular}


Table C-2. DC motor voltage (V) and current (A) at multiple rotational speeds against no load. Motor power $(W)$ was calculated from measured voltage, current. Motor torque $\left(\boldsymbol{\tau}_{D C}\right)$ was calculated from measured current and the derived motor torque constant, $K_{t}$.

\begin{tabular}{|ccccc|}
\hline Speed (RPM) & Voltage $(V)$ & Current $(A)$ & Power $(W)$ & $\begin{array}{c}\boldsymbol{\tau}_{D C} \\
K t^{*} I\left(N^{*} m m\right)\end{array}$ \\
\hline 500 & 2.291 & 0.222 & 0.51 & 7.009 \\
1000 & 3.902 & 0.235 & 0.92 & 7.419 \\
1500 & 5.525 & 0.250 & 1.38 & 7.892 \\
2000 & 7.162 & 0.272 & 1.95 & 8.587 \\
2500 & 8.770 & 0.288 & 2.53 & 9.092 \\
3000 & 10.323 & 0.299 & 3.09 & 9.439 \\
3500 & 11.942 & 0.308 & 3.68 & 9.724 \\
4000 & 13.442 & 0.321 & 4.31 & 10.134 \\
4500 & 15.040 & 0.331 & 4.98 & 10.450 \\
5000 & 16.566 & 0.342 & 5.67 & 10.797 \\
5500 & 18.215 & 0.350 & 6.38 & 11.049 \\
6000 & 19.754 & 0.360 & 7.11 & 11.365 \\
\hline
\end{tabular}

Table C-3. $D C$ motor voltage $(V)$ and current $(A)$ at multiple rotational speeds while driving the Pediatric VAD impeller without fluid. The total torque of the VAD without fluid ( $\boldsymbol{\tau}_{T o t a l}$ Dry $)$ was calculated from measured current and $K_{t}$. The torque due to friction ( $\boldsymbol{\tau}_{\text {Friction }}$ ) of the shaft and seal was determined as the difference between the total torque of the dry system ( $\boldsymbol{\tau}_{\text {Total, Dry }}$ ) and the total torque of the DC motor ( $\left.\boldsymbol{\tau}_{D C}\right)$.

\begin{tabular}{|cccccc|}
\hline $\begin{array}{c}\text { Speed } \\
(R P M)\end{array}$ & Voltage $(V)$ & Current $(A)$ & Power $(W)$ & $\begin{array}{c}\boldsymbol{\tau}_{\text {Total, Dry }} \\
K t^{*} /\left(N^{*} m m\right)\end{array}$ & $\begin{array}{c}\boldsymbol{\tau}_{\text {Friction }} \\
\text { Frictional Loss } \\
\left(N^{*} m m\right)\end{array}$ \\
\hline 1000 & 4.301 & 0.505 & 2.17 & 15.94 & 8.32 \\
2000 & 7.545 & 0.545 & 4.11 & 17.21 & 8.79 \\
3000 & 10.629 & 0.582 & 6.19 & 18.37 & 9.16 \\
4000 & 13.787 & 0.565 & 7.79 & 17.84 & 7.83 \\
5000 & 16.811 & 0.601 & 10.10 & 18.97 & 8.17 \\
6000 & 20.138 & 0.648 & 13.05 & 20.46 & 8.86 \\
\hline
\end{tabular}




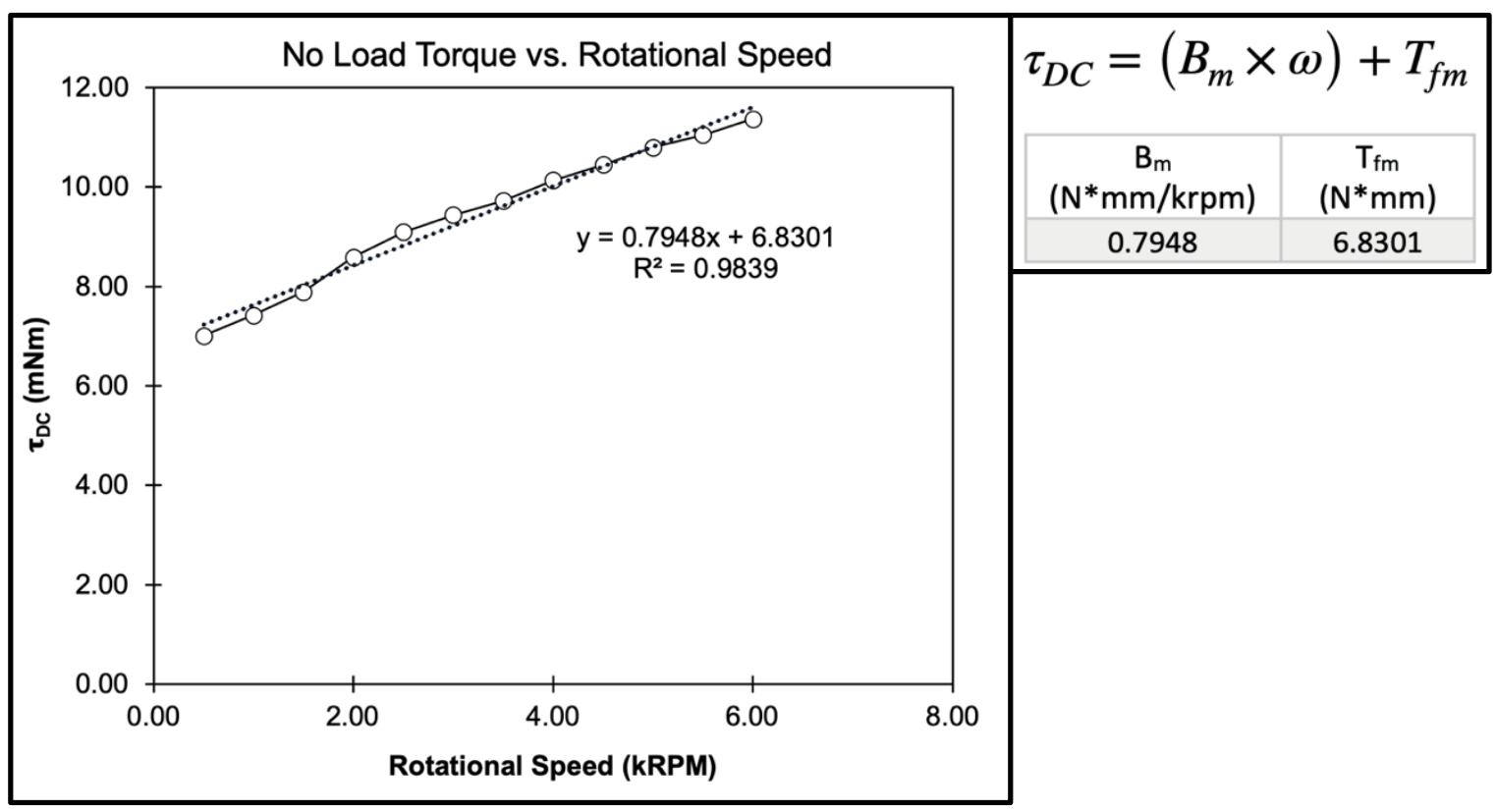

Figure C-1. Plot of calculated DC motor torque against no load versus rotational speed. The linear line of best fit and subsequent linear equation were used to determine the motor damping coefficient $\left(B_{m}\right)$ and the kinetic friction of the motor $\left(T_{f m}\right)$ and to calculate $D C$ motor torque $\left(\boldsymbol{\tau}_{D C}\right)$ at any rotational speed. 
Table C-4. Collected parameters of the Inspired Pediatric VAD in the Static Flow Loop (SFL) at 500 RPM. Total torque of the VAD in the SFL ( $\boldsymbol{\tau}_{\text {Total }}$ ) was calculated from measured current and $K_{t}$. The hydraulic torque ( $\boldsymbol{\tau}_{\text {Hydro }}$ ) of the impeller was the resulting net torque between the calculated total torque ( $\left.\boldsymbol{\tau}_{\text {Total }}\right)$ of the fluid filled system, the total base torque of the $D C$ motor $\left(\boldsymbol{\tau}_{D C}\right)$, and the torque due to friction of the shaft and seal ( $\boldsymbol{\tau}_{\text {Friction }}$ ). This was done for all rotational speeds (500 6000 RPM).

\begin{tabular}{|cccccccccccc|}
\hline $\begin{array}{c}\text { Speed } \\
(R P M)\end{array}$ & $\begin{array}{c}\text { VAD } \\
\text { Flow } \\
(\mathrm{L} / \mathrm{min})\end{array}$ & $\begin{array}{c}P 1 \\
(\mathrm{mmHg})\end{array}$ & $\begin{array}{c}P 2 \\
(\mathrm{mmHg})\end{array}$ & $\begin{array}{c}\text { Head } \\
(\mathrm{mmHg})\end{array}$ & $\begin{array}{c}\text { Voltage } \\
(\mathrm{V})\end{array}$ & $\begin{array}{c}\text { Current } \\
(A)\end{array}$ & $\begin{array}{c}\text { Power } \\
(W)\end{array}$ & $\begin{array}{c}\boldsymbol{\tau}_{\text {Total }} \\
\left(N^{*} m m\right)\end{array}$ & $\begin{array}{c}\boldsymbol{\tau}_{\text {Friction }} \\
\left(N^{*} m m\right)\end{array}$ & $\begin{array}{c}\boldsymbol{\tau}_{D C} \\
\left(N^{*} m m\right)\end{array}$ & $\begin{array}{c}\boldsymbol{\tau}_{\text {Hydro }} \\
\left(N^{*} m m\right)\end{array}$ \\
\hline 500 & 0.12 & 0.01 & 0.00 & 0.01 & 2.784 & 0.492 & 1.37 & 15.53 & 8.52 & 7.23 & -0.21 \\
500 & 0.00 & 2.00 & 0.50 & 1.50 & 2.778 & 0.508 & 1.41 & 16.04 & 8.52 & 7.23 & 0.29 \\
\hline
\end{tabular}

Table C-5. Collected parameters of the Inspired Pediatric VAD in the Static Flow Loop (SFL) at 1000 RPM.

\begin{tabular}{|cccccccccccc|}
\hline $\begin{array}{c}\text { Speed } \\
(R P M)\end{array}$ & $\begin{array}{c}\text { VAD } \\
\text { Flow } \\
(\mathrm{L} / \mathrm{min})\end{array}$ & $\begin{array}{c}P 1 \\
(\mathrm{mmHg})\end{array}$ & $\begin{array}{c}P 2 \\
(\mathrm{mmHg})\end{array}$ & $\begin{array}{c}\text { Head } \\
(\mathrm{mm} H \mathrm{H})\end{array}$ & $\begin{array}{c}\text { Voltage } \\
(\mathrm{V})\end{array}$ & $\begin{array}{c}\text { Current } \\
(\mathrm{A})\end{array}$ & $\begin{array}{c}\text { Power } \\
(\mathrm{W})\end{array}$ & $\begin{array}{c}\boldsymbol{\tau}_{\text {Total }} \\
\left(N^{*} m m\right)\end{array}$ & $\begin{array}{c}\boldsymbol{\tau}_{\text {Friction }} \\
\left(N^{*} m m\right)\end{array}$ & $\begin{array}{c}\boldsymbol{\tau}_{D C} \\
\left(N^{*} m m\right)\end{array}$ & $\begin{array}{c}\boldsymbol{\tau}_{\text {Hydro }} \\
\left(N^{*} m m\right)\end{array}$ \\
\hline 1000 & 0.35 & 1.30 & -0.92 & 2.22 & 4.483 & 0.506 & 2.27 & 15.97 & 8.52 & 7.62 & -0.17 \\
1000 & 0.15 & 7.63 & 0.37 & 7.26 & 4.484 & 0.501 & 2.25 & 15.82 & 8.52 & 7.62 & -0.33 \\
1000 & 0.00 & 10.30 & 0.85 & 9.45 & 4.485 & 0.499 & 2.24 & 15.75 & 8.52 & 7.62 & -0.39 \\
\hline
\end{tabular}

Table C-6. Collected parameters of the Inspired Pediatric VAD in the Static Flow Loop (SFL) at 1500 RPM.

\begin{tabular}{|c|c|c|c|c|c|c|c|c|c|c|c|}
\hline $\begin{array}{l}\text { Speed } \\
\text { (RPM) }\end{array}$ & $\begin{array}{c}\text { VAD } \\
\text { Flow } \\
\text { (L/min) }\end{array}$ & $\begin{array}{c}P 1 \\
(m m H g)\end{array}$ & $\begin{array}{c}P 2 \\
(m m H g)\end{array}$ & $\begin{array}{c}\text { Head } \\
(\mathrm{mmHg})\end{array}$ & $\begin{array}{l}\text { Voltage } \\
\text { (V) }\end{array}$ & $\begin{array}{c}\text { Current } \\
\text { (A) }\end{array}$ & $\begin{array}{l}\text { Power } \\
\text { (W) }\end{array}$ & $\begin{array}{c}\boldsymbol{\tau} \text { Total } \\
\left(N^{*} m m\right)\end{array}$ & 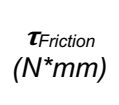 & $\begin{array}{c}\boldsymbol{\tau}_{D C} \\
\left(N^{*} m m\right)\end{array}$ & $\begin{array}{c}\boldsymbol{\tau} \text { Hydro } \\
\left(N^{*} m m\right)\end{array}$ \\
\hline 1500 & 0.67 & 2.44 & -3.51 & 5.95 & 6.186 & 0.526 & 3.25 & 16.61 & 8.52 & 8.02 & 0.06 \\
\hline 1500 & 0.40 & 12.82 & -1.03 & 13.85 & 6.188 & 0.520 & 3.22 & 16.42 & 8.52 & 8.02 & -0.13 \\
\hline 1500 & 0.20 & 19.89 & 0.39 & 19.50 & 6.192 & 0.513 & 3.18 & 16.20 & 8.52 & 8.02 & -0.35 \\
\hline 1500 & 0.00 & 24.20 & 1.06 & 23.14 & 6.192 & 0.510 & 3.16 & 16.10 & 8.52 & 8.02 & -0.44 \\
\hline
\end{tabular}


Table C-7. Collected parameters of the Inspired Pediatric VAD in the Static Flow Loop (SFL) at 2000 RPM.

\begin{tabular}{|c|c|c|c|c|c|c|c|c|c|c|c|}
\hline $\begin{array}{l}\text { Speed } \\
\text { (RPM) }\end{array}$ & $\begin{array}{c}\text { VAD } \\
\text { Flow } \\
(L / \text { min })\end{array}$ & $\begin{array}{c}P 1 \\
(m m H g)\end{array}$ & $\begin{array}{c}P 2 \\
(m m H g)\end{array}$ & $\begin{array}{c}\text { Head } \\
(\mathrm{mmHg})\end{array}$ & $\begin{array}{l}\text { Voltage } \\
\text { (V) }\end{array}$ & $\begin{array}{l}\text { Current } \\
\text { (A) }\end{array}$ & $\begin{array}{l}\text { Power } \\
\text { (W) }\end{array}$ & $\begin{array}{c}\boldsymbol{\tau}_{\text {Total }} \\
\left(N^{*} m m\right)\end{array}$ & $\begin{array}{c}\boldsymbol{\tau}_{\text {Friction }} \\
\left(N^{*} m m\right)\end{array}$ & $\begin{array}{c}\boldsymbol{\tau}_{D C} \\
\left(N^{*} m m\right)\end{array}$ & $\begin{array}{c}\boldsymbol{\tau}_{\text {Hydro }} \\
\left(N^{*} m m\right)\end{array}$ \\
\hline 2000 & 0.99 & 3.87 & -7.65 & 11.52 & 7.843 & 0.550 & 4.31 & 17.36 & 8.52 & 8.42 & 0.42 \\
\hline 2000 & 0.80 & 14.89 & -4.84 & 19.73 & 7.846 & 0.544 & 4.27 & 17.17 & 8.52 & 8.42 & 0.23 \\
\hline 2000 & 0.60 & 24.41 & -2.60 & 27.01 & 7.847 & 0.539 & 4.23 & 17.02 & 8.52 & 8.42 & 0.08 \\
\hline 2000 & 0.40 & 32.79 & -0.62 & 33.41 & 7.849 & 0.535 & 4.20 & 16.89 & 8.52 & 8.42 & -0.05 \\
\hline 2000 & 0.20 & 39.01 & 0.62 & 38.39 & 7.850 & 0.532 & 4.18 & 16.80 & 8.52 & 8.42 & -0.14 \\
\hline 2000 & 0.00 & 45.23 & 1.32 & 43.91 & 7.852 & 0.526 & 4.13 & 16.61 & 8.52 & 8.42 & -0.33 \\
\hline
\end{tabular}

Table C-8. Collected parameters of the Inspired Pediatric VAD in the Static Flow Loop (SFL) at 2500 RPM.

\begin{tabular}{|ccccccccccccc|}
\hline $\begin{array}{c}\text { Speed } \\
(R P M)\end{array}$ & $\begin{array}{c}\text { VAD } \\
\text { Flow } \\
(\mathrm{L} / \mathrm{min})\end{array}$ & $\begin{array}{c}P 1 \\
(\mathrm{mmHg})\end{array}$ & $\begin{array}{c}P 2 \\
(\mathrm{mmHg})\end{array}$ & $\begin{array}{c}\text { Head } \\
(\mathrm{mmHg})\end{array}$ & $\begin{array}{c}\text { Voltage } \\
(\mathrm{V})\end{array}$ & $\begin{array}{c}\text { Current } \\
(\mathrm{A})\end{array}$ & $\begin{array}{c}\text { Power } \\
(W)\end{array}$ & $\begin{array}{c}\boldsymbol{\tau}_{\text {Total }} \\
\left(N^{*} m m\right)\end{array}$ & $\begin{array}{c}\boldsymbol{\tau}_{\text {Friction }} \\
\left(N^{*} m m^{*}\right)\end{array}$ & $\begin{array}{c}\boldsymbol{\tau}_{D C} \\
\left(N^{*} m m\right)\end{array}$ & $\begin{array}{c}\boldsymbol{\tau}_{\text {Hydro }} \\
\left(N^{*} m m\right)\end{array}$ \\
\hline 2500 & 1.33 & 3.87 & -13.55 & 17.42 & 9.499 & 0.598 & 5.68 & 18.88 & 8.52 & 8.82 & 1.54 \\
2500 & 1.00 & 27.27 & -7.50 & 34.77 & 9.502 & 0.593 & 5.63 & 18.72 & 8.52 & 8.82 & 1.38 \\
2500 & 0.75 & 40.32 & -4.03 & 44.35 & 9.505 & 0.588 & 5.59 & 18.56 & 8.52 & 8.82 & 1.23 \\
2500 & 0.50 & 52.30 & -1.23 & 53.53 & 9.507 & 0.580 & 5.51 & 18.31 & 8.52 & 8.82 & 0.97 \\
2500 & 0.25 & 60.60 & 0.68 & 59.92 & 9.509 & 0.576 & 5.48 & 18.18 & 8.52 & 8.82 & 0.85 \\
2500 & 0.00 & 69.19 & 1.62 & 67.57 & 9.510 & 0.571 & 5.43 & 18.03 & 8.52 & 8.82 & 0.69 \\
\hline
\end{tabular}


Table C-9. Collected parameters of the Inspired Pediatric VAD in the Static Flow Loop (SFL) at 3000 RPM.

\begin{tabular}{|c|c|c|c|c|c|c|c|c|c|c|c|}
\hline $\begin{array}{l}\text { Speed } \\
\text { (RPM) }\end{array}$ & $\begin{array}{l}\text { VAD } \\
\text { Flow } \\
\text { (L/min) }\end{array}$ & $\begin{array}{c}P 1 \\
(m m H g)\end{array}$ & $\begin{array}{c}P 2 \\
(m m H g)\end{array}$ & $\begin{array}{c}\text { Head } \\
(\mathrm{mmHg})\end{array}$ & $\begin{array}{l}\text { Voltage } \\
\text { (V) }\end{array}$ & $\begin{array}{l}\text { Current } \\
\text { (A) }\end{array}$ & $\begin{array}{l}\text { Power } \\
\text { (W) }\end{array}$ & $\begin{array}{c}\boldsymbol{\tau}_{\text {Total }} \\
\left(N^{*} m m\right)\end{array}$ & $\begin{array}{c}\boldsymbol{\tau}_{\text {Friction }} \\
\left(N^{*} m m\right)\end{array}$ & $\begin{array}{c}\boldsymbol{\tau}_{D C} \\
\left(N^{*} m m\right)\end{array}$ & $\begin{array}{c}\boldsymbol{\tau}_{\text {Hydro }} \\
\left(N^{*} m m\right)\end{array}$ \\
\hline 3000 & 1.70 & 5.41 & -21.94 & 27.35 & 11.131 & 0.661 & 7.36 & 20.87 & 8.52 & 9.21 & 3.13 \\
\hline 3000 & 1.50 & 21.22 & -17.44 & 38.66 & 11.132 & 0.655 & 7.29 & 20.68 & 8.52 & 9.21 & 2.94 \\
\hline 3000 & 1.25 & 41.75 & -11.78 & 53.53 & 11.135 & 0.650 & 7.24 & 20.52 & 8.52 & 9.21 & 2.79 \\
\hline 3000 & 1.00 & 58.73 & -7.65 & 66.38 & 11.137 & 0.643 & 7.16 & 20.30 & 8.52 & 9.21 & 2.56 \\
\hline 3000 & 0.75 & 72.27 & -3.93 & 76.20 & 11.142 & 0.634 & 7.06 & 20.02 & 8.52 & 9.21 & 2.28 \\
\hline 3000 & 0.50 & 85.44 & -0.90 & 86.34 & 11.139 & 0.633 & 7.05 & 19.98 & 8.52 & 9.21 & 2.25 \\
\hline 3000 & 0.25 & 93.33 & 0.73 & 92.60 & 11.143 & 0.630 & 7.02 & 19.89 & 8.52 & 9.21 & 2.15 \\
\hline 3000 & 0.00 & 100.33 & 1.80 & 98.53 & 11.145 & 0.627 & 6.99 & 19.79 & 8.52 & 9.21 & 2.06 \\
\hline
\end{tabular}

Table C-10. Collected parameters of the Inspired Pediatric VAD in the Static Flow Loop (SFL) at 3500 RPM.

\begin{tabular}{|c|c|c|c|c|c|c|c|c|c|c|c|}
\hline $\begin{array}{l}\text { Speed } \\
\text { (RPM) }\end{array}$ & $\begin{array}{c}\text { VAD } \\
\text { Flow } \\
\text { (L/min) }\end{array}$ & $\begin{array}{c}P 1 \\
(m m H g)\end{array}$ & $\begin{array}{c}P 2 \\
(m m H g)\end{array}$ & $\begin{array}{c}\text { Head } \\
(\mathrm{mmHg})\end{array}$ & $\begin{array}{l}\text { Voltage } \\
\text { (V) }\end{array}$ & $\begin{array}{l}\text { Current } \\
\text { (A) }\end{array}$ & $\begin{array}{l}\text { Power } \\
\text { (W) }\end{array}$ & $\begin{array}{c}\boldsymbol{\tau} \text { Total } \\
\left(N^{*} m m\right)\end{array}$ & $\begin{array}{c}\boldsymbol{\tau} \text { - } \text {-riction } \\
\left(N^{*} m m\right)\end{array}$ & $\begin{array}{c}\boldsymbol{\tau}_{D C} \\
\left(N^{*} m m\right)\end{array}$ & $\begin{array}{c}\boldsymbol{\tau} \text { Hydro } \\
\left(N^{*} m m\right)\end{array}$ \\
\hline 3500 & 2.10 & 5.67 & -32.99 & 38.66 & 12.768 & 0.723 & 9.23 & 22.83 & 8.52 & 9.61 & 4.69 \\
\hline 3500 & 2.00 & 13.70 & -30.99 & 44.69 & 12.770 & 0.717 & 9.16 & 22.64 & 8.52 & 9.61 & 4.50 \\
\hline 3500 & 1.75 & 37.20 & -23.43 & 60.63 & 12.773 & 0.714 & 9.12 & 22.54 & 8.52 & 9.61 & 4.41 \\
\hline 3500 & 1.50 & 58.71 & -17.19 & 75.90 & 12.776 & 0.706 & 9.02 & 22.29 & 8.52 & 9.61 & 4.16 \\
\hline 3500 & 1.25 & 77.48 & -11.90 & 89.38 & 12.780 & 0.698 & 8.92 & 22.04 & 8.52 & 9.61 & 3.90 \\
\hline 3500 & 1.00 & 93.35 & -7.32 & 100.67 & 12.776 & 0.705 & 9.01 & 22.26 & 8.52 & 9.61 & 4.12 \\
\hline 3500 & 0.75 & 107.25 & -3.78 & 111.03 & 12.800 & 0.720 & 9.22 & 22.73 & 8.52 & 9.61 & 4.60 \\
\hline 3500 & 0.50 & 119.94 & -1.09 & 121.03 & 12.805 & 0.721 & 9.23 & 22.76 & 8.52 & 9.61 & 4.63 \\
\hline 3500 & 0.25 & 130.07 & 1.00 & 129.07 & 12.806 & 0.715 & 9.16 & 22.57 & 8.52 & 9.61 & 4.44 \\
\hline 3500 & 0.00 & 139.68 & 1.95 & 137.73 & 12.808 & 0.715 & 9.16 & 22.57 & 8.52 & 9.61 & 4.44 \\
\hline
\end{tabular}


Table C-11. Collected parameters of the Inspired Pediatric VAD in the Static Flow Loop (SFL) at 4000 RPM.

\begin{tabular}{|c|c|c|c|c|c|c|c|c|c|c|c|}
\hline $\begin{array}{l}\text { Speed } \\
\text { (RPM) }\end{array}$ & $\begin{array}{l}\text { VAD } \\
\text { Flow } \\
\text { (L/min) }\end{array}$ & $\begin{array}{c}P 1 \\
(m m H g)\end{array}$ & $\begin{array}{c}P 2 \\
(m m H g)\end{array}$ & $\begin{array}{c}\text { Head } \\
(\mathrm{mmHg})\end{array}$ & $\begin{array}{l}\text { Voltage } \\
\text { (V) }\end{array}$ & $\begin{array}{l}\text { Current } \\
\text { (A) }\end{array}$ & $\begin{array}{l}\text { Power } \\
\text { (W) }\end{array}$ & $\begin{array}{c}\boldsymbol{\tau}_{\text {Total }} \\
\left(N^{*} m m\right)\end{array}$ & $\begin{array}{c}\boldsymbol{\tau}_{\text {Friction }} \\
\left(N^{*} m m\right)\end{array}$ & $\begin{array}{c}\boldsymbol{\tau}_{D C} \\
\left(N^{*} m m\right)\end{array}$ & $\begin{array}{c}\boldsymbol{\tau}_{\text {Hydro }} \\
\left(N^{*} m m\right)\end{array}$ \\
\hline 4000 & 2.48 & 6.70 & -46.91 & 53.61 & 14.491 & 0.854 & 12.38 & 26.96 & 8.52 & 10.01 & 8.43 \\
\hline 4000 & 2.25 & 32.66 & -38.20 & 70.86 & 14.480 & 0.868 & 12.57 & 27.40 & 8.52 & 10.01 & 8.87 \\
\hline 4000 & 2.00 & 55.86 & -31.23 & 87.09 & 14.488 & 0.861 & 12.47 & 27.18 & 8.52 & 10.01 & 8.65 \\
\hline 4000 & 1.75 & 82.31 & -23.24 & 105.55 & 14.494 & 0.841 & 12.19 & 26.55 & 8.52 & 10.01 & 8.02 \\
\hline 4000 & 1.50 & 103.47 & -16.86 & 120.33 & 14.496 & 0.839 & 12.16 & 26.49 & 8.52 & 10.01 & 7.96 \\
\hline 4000 & 1.25 & 121.38 & -11.64 & 133.02 & 14.499 & 0.833 & 12.08 & 26.30 & 8.52 & 10.01 & 7.77 \\
\hline 4000 & 1.00 & 138.64 & -7.23 & 145.87 & 14.500 & 0.828 & 12.01 & 26.14 & 8.52 & 10.01 & 7.61 \\
\hline 4000 & 0.75 & 154.45 & -3.40 & 157.85 & 14.502 & 0.826 & 11.98 & 26.08 & 8.52 & 10.01 & 7.55 \\
\hline 4000 & 0.50 & 165.10 & -0.64 & 165.74 & 14.504 & 0.822 & 11.92 & 25.95 & 8.52 & 10.01 & 7.42 \\
\hline 4000 & 0.25 & 174.40 & 1.30 & 173.10 & 14.504 & 0.821 & 11.91 & 25.92 & 8.52 & 10.01 & 7.39 \\
\hline 4000 & 0.00 & 182.24 & 2.23 & 180.01 & 14.507 & 0.815 & 11.82 & 25.73 & 8.52 & 10.01 & 7.20 \\
\hline
\end{tabular}


Table C-12. Collected parameters of the Inspired Pediatric VAD in the Static Flow Loop (SFL) at 4500 RPM.

\begin{tabular}{|cccccccccccc|}
\hline $\begin{array}{c}\text { Speed } \\
(R P M)\end{array}$ & $\begin{array}{c}\text { VAD } \\
\text { Flow } \\
(\mathrm{L} / \mathrm{min})\end{array}$ & $\begin{array}{c}P 1 \\
(\mathrm{mmHg})\end{array}$ & $\begin{array}{c}P 2 \\
(\mathrm{~mm} H \mathrm{Hg})\end{array}$ & $\begin{array}{c}\mathrm{Head} \\
(\mathrm{mmHg})\end{array}$ & $\begin{array}{c}\text { Voltage } \\
(\mathrm{V})\end{array}$ & $\begin{array}{c}\text { Current } \\
(\mathrm{A})\end{array}$ & $\begin{array}{c}\text { Power } \\
(W)\end{array}$ & $\begin{array}{c}\boldsymbol{\tau}_{\text {Total }} \\
\left(N^{*} m m\right)\end{array}$ & $\begin{array}{c}\boldsymbol{\tau}_{\text {Friction }}\left(N^{*} m m^{*}\right) \\
\left(N^{*} m m\right)\end{array}$ & $\begin{array}{c}\boldsymbol{\tau}_{D C} \\
\left(N^{*} m m\right)\end{array}$ \\
\hline 4500 & 2.91 & 4.57 & -63.52 & 68.09 & 16.063 & 0.941 & 15.12 & 29.71 & 8.52 & 10.41 & 10.78 \\
4500 & 2.75 & 24.49 & -56.84 & 81.33 & 16.071 & 0.935 & 15.03 & 29.52 & 8.52 & 10.41 & 10.59 \\
4500 & 2.50 & 53.71 & -47.99 & 101.70 & 16.073 & 0.934 & 15.01 & 29.49 & 8.52 & 10.41 & 10.56 \\
4500 & 2.25 & 81.12 & -38.70 & 119.82 & 16.079 & 0.918 & 14.76 & 28.98 & 8.52 & 10.41 & 10.05 \\
4500 & 2.00 & 110.86 & -29.57 & 140.43 & 16.082 & 0.911 & 14.65 & 28.76 & 8.52 & 10.41 & 9.83 \\
4500 & 1.75 & 132.06 & -23.01 & 155.07 & 16.087 & 0.900 & 14.48 & 28.41 & 8.52 & 10.41 & 9.49 \\
4500 & 1.50 & 155.45 & -16.09 & 171.54 & 16.090 & 0.891 & 14.34 & 28.13 & 8.52 & 10.41 & 9.20 \\
4500 & 1.25 & 171.55 & -11.14 & 182.69 & 16.093 & 0.888 & 14.29 & 28.03 & 8.52 & 10.41 & 9.11 \\
4500 & 1.00 & 186.33 & -6.91 & 193.24 & 16.098 & 0.875 & 14.09 & 27.62 & 8.52 & 10.41 & 8.70 \\
4500 & 0.75 & 202.93 & -2.82 & 205.75 & 16.101 & 0.870 & 14.01 & 27.47 & 8.52 & 10.41 & 8.54 \\
4500 & 0.50 & 215.46 & -0.38 & 215.84 & 16.104 & 0.865 & 13.93 & 27.31 & 8.52 & 10.41 & 8.38 \\
4500 & 0.25 & 223.70 & 1.49 & 222.21 & 16.106 & 0.860 & 13.85 & 27.15 & 8.52 & 10.41 & 8.22 \\
4500 & 0.00 & 233.31 & 2.43 & 230.88 & 16.107 & 0.855 & 13.77 & 26.99 & 8.52 & 10.41 & 8.07 \\
\hline
\end{tabular}


Table C-13. Collected parameters of the Inspired Pediatric VAD in the Static Flow Loop (SFL) at 5000 RPM.

\begin{tabular}{|c|c|c|c|c|c|c|c|c|c|c|c|}
\hline $\begin{array}{l}\text { Speed } \\
\text { (RPM) }\end{array}$ & $\begin{array}{l}\text { VAD } \\
\text { Flow } \\
\text { (L/min) }\end{array}$ & $\begin{array}{c}P 1 \\
(m m H g)\end{array}$ & $\begin{array}{c}P 2 \\
(m m H g)\end{array}$ & $\begin{array}{c}\text { Head } \\
(\mathrm{mmHg})\end{array}$ & $\begin{array}{l}\text { Voltage } \\
\text { (V) }\end{array}$ & $\begin{array}{l}\text { Current } \\
\text { (A) }\end{array}$ & $\begin{array}{l}\text { Power } \\
\text { (W) }\end{array}$ & $\begin{array}{c}\boldsymbol{\tau}_{\text {Total }} \\
\left(N^{*} m m\right)\end{array}$ & $\begin{array}{c}\boldsymbol{\tau}_{\text {Friction }} \\
\left(N^{*} m m\right)\end{array}$ & $\begin{array}{c}\boldsymbol{\tau}_{D C} \\
\left(N^{*} m m\right)\end{array}$ & $\begin{array}{c}\boldsymbol{\tau}_{\text {Hydro }} \\
\left(N^{*} m m\right)\end{array}$ \\
\hline 5000 & 3.39 & 4.57 & -82.18 & 86.75 & 17.663 & 1.002 & 17.70 & 31.63 & 8.52 & 10.80 & 12.31 \\
\hline 5000 & 3.25 & 25.63 & -75.62 & 101.25 & 17.667 & 0.998 & 17.63 & 31.51 & 8.52 & 10.80 & 12.18 \\
\hline 5000 & 3.00 & 57.67 & -65.33 & 123.00 & 17.668 & 0.991 & 17.51 & 31.29 & 8.52 & 10.80 & 11.96 \\
\hline 5000 & 2.75 & 85.84 & -55.68 & 141.52 & 17.675 & 0.980 & 17.32 & 30.94 & 8.52 & 10.80 & 11.61 \\
\hline 5000 & 2.50 & 119.47 & -44.85 & 164.32 & 17.677 & 0.974 & 17.22 & 30.75 & 8.52 & 10.80 & 11.42 \\
\hline 5000 & 2.25 & 144.61 & -36.50 & 181.11 & 17.684 & 0.961 & 16.99 & 30.34 & 8.52 & 10.80 & 11.01 \\
\hline 5000 & 2.00 & 168.92 & -29.36 & 198.28 & 17.685 & 0.958 & 16.94 & 30.24 & 8.52 & 10.80 & 10.92 \\
\hline 5000 & 1.75 & 190.31 & -22.13 & 212.44 & 17.689 & 0.946 & 16.73 & 29.87 & 8.52 & 10.80 & 10.54 \\
\hline 5000 & 1.50 & 214.02 & -16.42 & 230.44 & 17.686 & 0.957 & 16.93 & 30.21 & 8.52 & 10.80 & 10.89 \\
\hline 5000 & 1.25 & 231.02 & -10.61 & 241.63 & 17.689 & 0.949 & 16.79 & 29.96 & 8.52 & 10.80 & 10.64 \\
\hline 5000 & 1.00 & 244.80 & -6.91 & 251.71 & 17.691 & 0.941 & 16.65 & 29.71 & 8.52 & 10.80 & 10.38 \\
\hline 5000 & 0.75 & 260.31 & -2.89 & 263.20 & 17.698 & 0.928 & 16.42 & 29.30 & 8.52 & 10.80 & 9.97 \\
\hline 5000 & 0.50 & 273.43 & -0.20 & 273.63 & 17.700 & 0.922 & 16.32 & 29.11 & 8.52 & 10.80 & 9.78 \\
\hline 5000 & 0.25 & 284.98 & 1.68 & 283.30 & 17.704 & 0.908 & 16.08 & 28.67 & 8.52 & 10.80 & 9.34 \\
\hline 5000 & 0.00 & 296.36 & 2.64 & 293.72 & 17.711 & 0.892 & 15.80 & 28.16 & 8.52 & 10.80 & 8.84 \\
\hline
\end{tabular}


Table C-14. Collected parameters of the Inspired Pediatric VAD in the Static Flow Loop (SFL) at 5500 RPM.

\begin{tabular}{|c|c|c|c|c|c|c|c|c|c|c|c|}
\hline $\begin{array}{l}\text { Speed } \\
\text { (RPM) }\end{array}$ & $\begin{array}{c}\text { VAD } \\
\text { Flow } \\
\text { (L/min) }\end{array}$ & $\begin{array}{c}P 1 \\
(m m H g)\end{array}$ & $\begin{array}{c}P 2 \\
(m m H g)\end{array}$ & $\begin{array}{c}\text { Head } \\
(\mathrm{mmHg})\end{array}$ & $\begin{array}{l}\text { Voltage } \\
\text { (V) }\end{array}$ & $\begin{array}{l}\text { Current } \\
\text { (A) }\end{array}$ & $\begin{array}{l}\text { Power } \\
(W)\end{array}$ & $\begin{array}{c}\boldsymbol{\tau}_{\text {Total }} \\
\left(N^{*} m m\right)\end{array}$ & $\begin{array}{c}\boldsymbol{\tau}_{\text {Friction }} \\
\left(N^{*} m m\right)\end{array}$ & $\begin{array}{c}\boldsymbol{\tau}_{D C} \\
\left(N^{*} m m\right)\end{array}$ & $\begin{array}{c}\boldsymbol{\tau}_{\text {Hydro }} \\
\left(N^{*} m m\right)\end{array}$ \\
\hline 5500 & 3.88 & -0.88 & -103.21 & 102.33 & 19.063 & 1.072 & 20.44 & 33.84 & 8.52 & 11.20 & 14.12 \\
\hline 5500 & 3.75 & 13.44 & -97.87 & 111.31 & 19.033 & 1.066 & 20.29 & 33.65 & 8.52 & 11.20 & 13.93 \\
\hline 5500 & 3.50 & 48.42 & -86.29 & 134.71 & 19.073 & 1.063 & 20.27 & 33.56 & 8.52 & 11.20 & 13.84 \\
\hline 5500 & 3.25 & 83.78 & -73.58 & 157.36 & 19.040 & 1.060 & 20.18 & 33.46 & 8.52 & 11.20 & 13.74 \\
\hline 5500 & 3.00 & 115.27 & -63.36 & 178.63 & 19.060 & 1.051 & 20.03 & 33.18 & 8.52 & 11.20 & 13.46 \\
\hline 5500 & 2.75 & 148.17 & -53.52 & 201.69 & 19.051 & 1.045 & 19.91 & 32.99 & 8.52 & 11.20 & 13.27 \\
\hline 5500 & 2.50 & 178.38 & -44.18 & 222.56 & 19.031 & 1.039 & 19.77 & 32.80 & 8.52 & 11.20 & 13.08 \\
\hline 5500 & 2.25 & 205.89 & -35.63 & 241.52 & 19.063 & 1.025 & 19.54 & 32.36 & 8.52 & 11.20 & 12.64 \\
\hline 5500 & 2.00 & 228.62 & -28.66 & 257.28 & 19.073 & 1.018 & 19.42 & 32.14 & 8.52 & 11.20 & 12.42 \\
\hline 5500 & 1.75 & 253.44 & -21.91 & 275.35 & 19.081 & 1.011 & 19.29 & 31.92 & 8.52 & 11.20 & 12.20 \\
\hline 5500 & 1.50 & 273.20 & -15.24 & 288.44 & 19.083 & 1.006 & 19.20 & 31.76 & 8.52 & 11.20 & 12.04 \\
\hline 5500 & 1.25 & 290.32 & -10.29 & 300.61 & 19.095 & 0.994 & 18.98 & 31.38 & 8.52 & 11.20 & 11.66 \\
\hline 5500 & 1.00 & 310.60 & -5.98 & 316.58 & 19.100 & 0.985 & 18.81 & 31.10 & 8.52 & 11.20 & 11.37 \\
\hline 5500 & 0.75 & 319.54 & -2.54 & 322.08 & 19.106 & 0.972 & 18.57 & 30.69 & 8.52 & 11.20 & 10.96 \\
\hline 5500 & 0.50 & 335.32 & 0.27 & 335.05 & 19.111 & 0.956 & 18.27 & 30.18 & 8.52 & 11.20 & 10.46 \\
\hline 5500 & 0.25 & 345.77 & 2.00 & 343.77 & 19.118 & 0.954 & 18.24 & 30.12 & 8.52 & 11.20 & 10.40 \\
\hline 5500 & 0.00 & 357.41 & 2.89 & 354.52 & 19.124 & 0.937 & 17.92 & 29.58 & 8.52 & 11.20 & 9.86 \\
\hline
\end{tabular}


Table C-15. Collected parameters of the Inspired Pediatric VAD in the Static Flow Loop (SFL) at 6000 RPM.

\begin{tabular}{|c|c|c|c|c|c|c|c|c|c|c|c|}
\hline $\begin{array}{l}\text { Speed } \\
\text { (RPM) }\end{array}$ & $\begin{array}{c}V A D \\
\text { Flow } \\
\text { (L/min) }\end{array}$ & $\begin{array}{c}P 1 \\
(m m H g)\end{array}$ & $\begin{array}{c}P 2 \\
(m m H g)\end{array}$ & $\begin{array}{c}\text { Head } \\
(\mathrm{mmHg})\end{array}$ & $\begin{array}{l}\text { Voltage } \\
\text { (V) }\end{array}$ & $\begin{array}{l}\text { Current } \\
\text { (A) }\end{array}$ & $\begin{array}{l}\text { Power } \\
\text { (W) }\end{array}$ & $\begin{array}{c}\boldsymbol{\tau}_{\text {Total }} \\
\left(N^{*} m m\right)\end{array}$ & $\begin{array}{c}\boldsymbol{\tau}_{\text {Friction }} \\
\left(N^{*} m m\right)\end{array}$ & $\begin{array}{c}\boldsymbol{\tau}_{D C} \\
\left(N^{*} m m\right)\end{array}$ & $\begin{array}{c}\boldsymbol{\tau}_{\text {Hydro }} \\
\left(N^{*} m m\right)\end{array}$ \\
\hline 6000 & 4.27 & 0.64 & -125.91 & 126.55 & 20.574 & 1.171 & 24.09 & 36.97 & 8.52 & 11.60 & 16.85 \\
\hline 6000 & 4.00 & 46.05 & -111.75 & 157.80 & 20.588 & 1.151 & 23.70 & 36.34 & 8.52 & 11.60 & 16.22 \\
\hline 6000 & 3.75 & 85.74 & -98.87 & 184.61 & 20.593 & 1.143 & 23.54 & 36.08 & 8.52 & 11.60 & 15.97 \\
\hline 6000 & 3.50 & 123.43 & -87.34 & 210.77 & 20.598 & 1.130 & 23.28 & 35.67 & 8.52 & 11.60 & 15.56 \\
\hline 6000 & 3.25 & 160.23 & -73.63 & 233.86 & 20.602 & 1.118 & 23.03 & 35.30 & 8.52 & 11.60 & 15.18 \\
\hline 6000 & 3.00 & 191.58 & -63.77 & 255.35 & 20.606 & 1.104 & 22.75 & 34.85 & 8.52 & 11.60 & 14.73 \\
\hline 6000 & 2.75 & 225.04 & -53.04 & 278.08 & 20.610 & 1.098 & 22.63 & 34.66 & 8.52 & 11.60 & 14.54 \\
\hline 6000 & 2.50 & 252.29 & -43.69 & 295.98 & 20.616 & 1.080 & 22.27 & 34.10 & 8.52 & 11.60 & 13.98 \\
\hline 6000 & 2.25 & 278.03 & -35.56 & 313.59 & 20.620 & 1.074 & 22.15 & 33.91 & 8.52 & 11.60 & 13.79 \\
\hline 6000 & 2.00 & 300.32 & -28.48 & 328.80 & 20.623 & 1.063 & 21.92 & 33.56 & 8.52 & 11.60 & 13.44 \\
\hline 6000 & 1.75 & 321.99 & -21.34 & 343.33 & 20.624 & 1.055 & 21.76 & 33.31 & 8.52 & 11.60 & 13.19 \\
\hline 6000 & 1.50 & 338.96 & -15.51 & 354.47 & 20.623 & 1.065 & 21.96 & 33.62 & 8.52 & 11.60 & 13.50 \\
\hline 6000 & 1.25 & 356.79 & -10.27 & 367.06 & 20.625 & 1.055 & 21.76 & 33.31 & 8.52 & 11.60 & 13.19 \\
\hline 6000 & 1.00 & 372.32 & -6.07 & 378.39 & 20.630 & 1.046 & 21.58 & 33.02 & 8.52 & 11.60 & 12.90 \\
\hline 6000 & 0.75 & 388.75 & -2.65 & 391.40 & 20.635 & 1.033 & 21.32 & 32.61 & 8.52 & 11.60 & 12.49 \\
\hline 6000 & 0.50 & 402.07 & 0.24 & 401.83 & 20.638 & 1.019 & 21.03 & 32.17 & 8.52 & 11.60 & 12.05 \\
\hline 6000 & 0.25 & 412.88 & 2.04 & 410.84 & 20.645 & 0.996 & 20.56 & 31.44 & 8.52 & 11.60 & 11.32 \\
\hline 6000 & 0.00 & 425.41 & 2.98 & 422.43 & 20.656 & 0.962 & 19.87 & 30.37 & 8.52 & 11.60 & 10.25 \\
\hline
\end{tabular}


Figure C-2. Calibrated Cannon-Fenske style viscometer submerged in a $37^{\circ} \mathrm{C}$ water bath to measure the viscosity of the blood analogue solution. sample

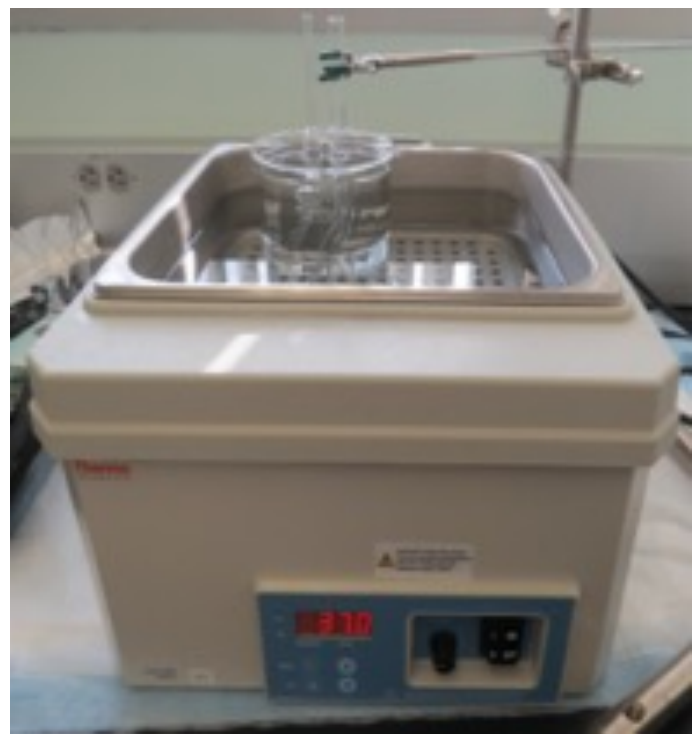

Figure C-3. (Left) Laser-based tachometer to measure impeller rotational speed, (Right) PMDC motor and shaft with coupler attached to SLA printed pump impeller.
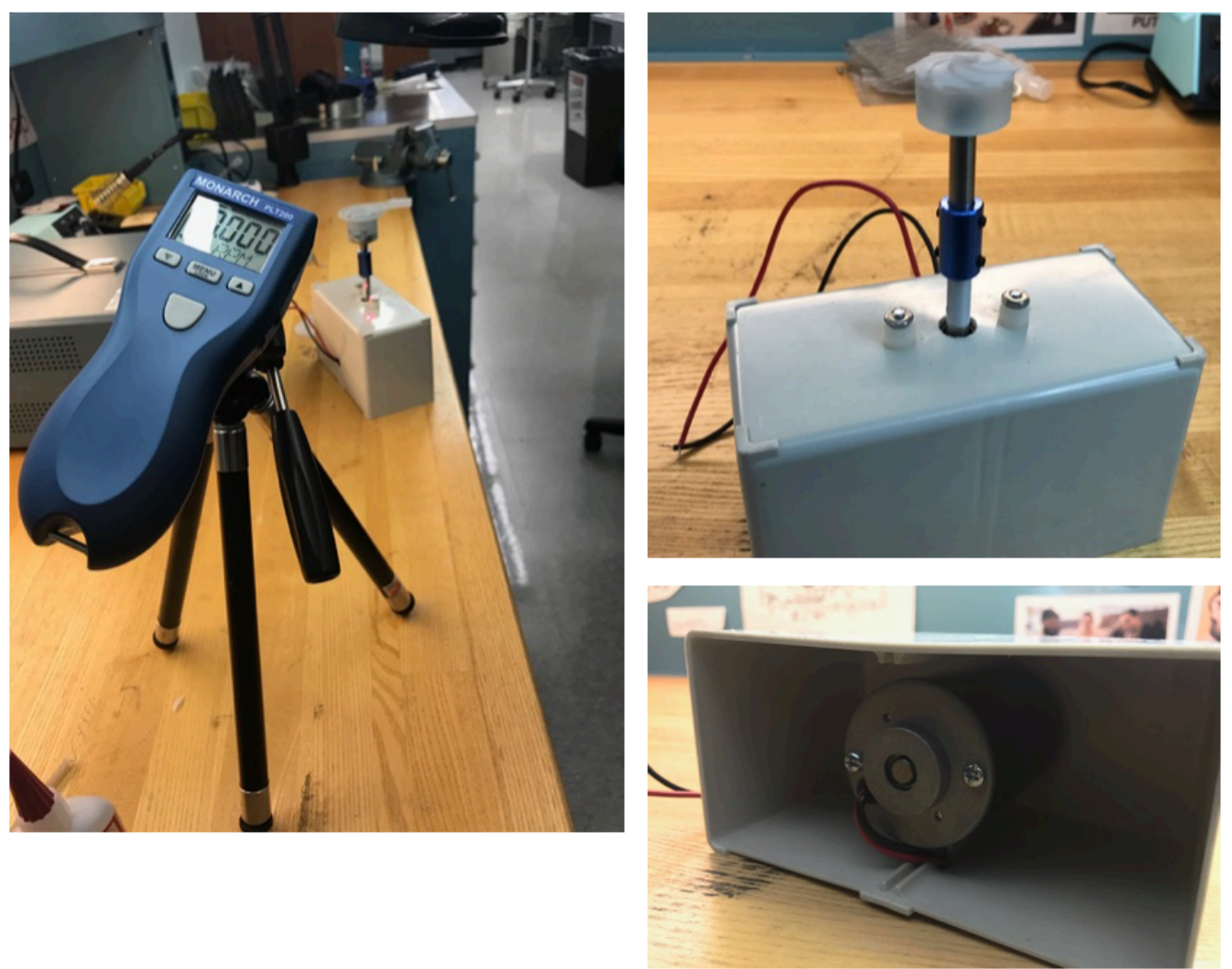
Figure C-4. Various images of static flow loop (SFL) construction with a view (Bottom Right) of the variable DC power supply, multimeters, and $D A Q$ data collection system.
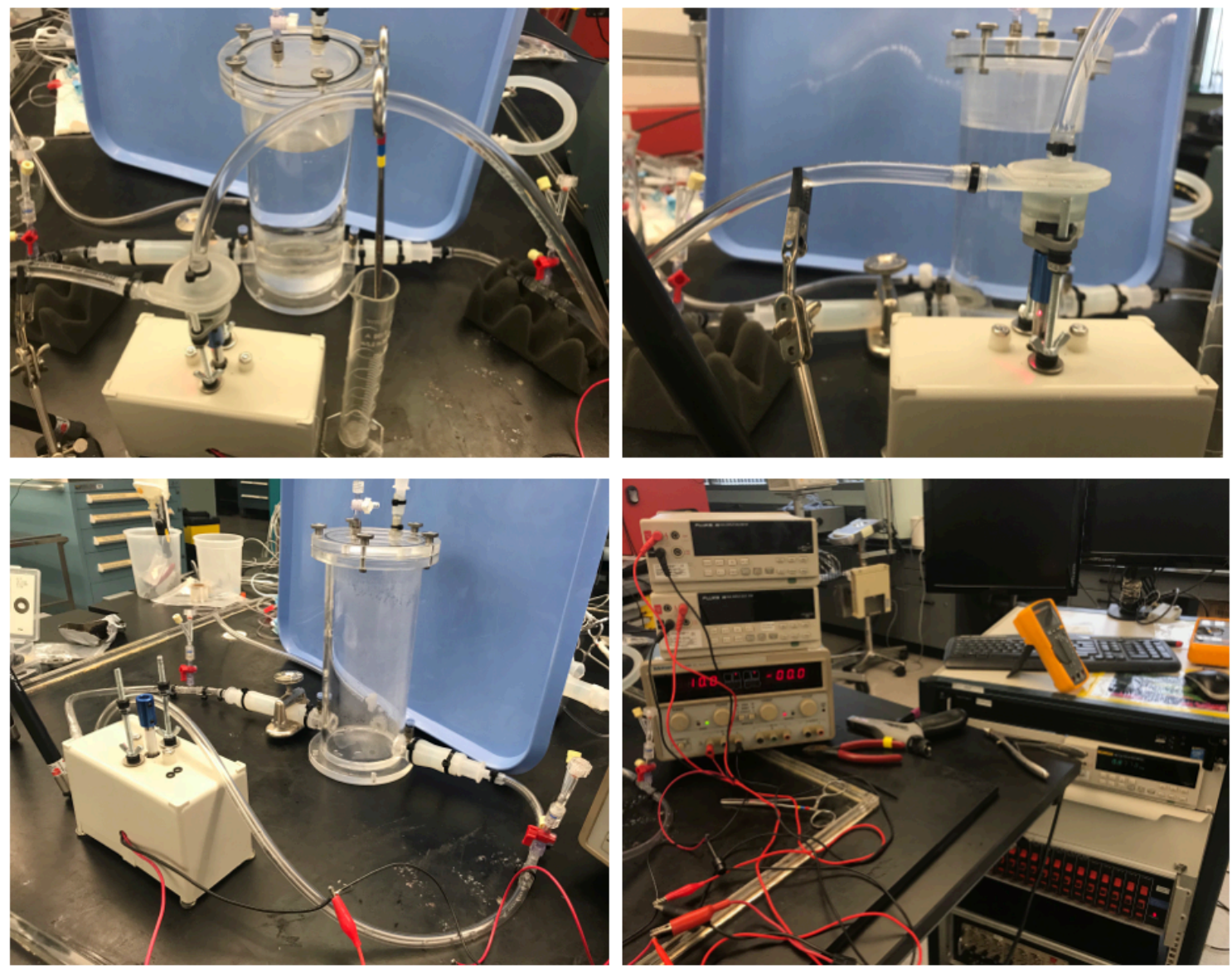
Figure C-5. View of the completed static flow loop system with heated water bath.

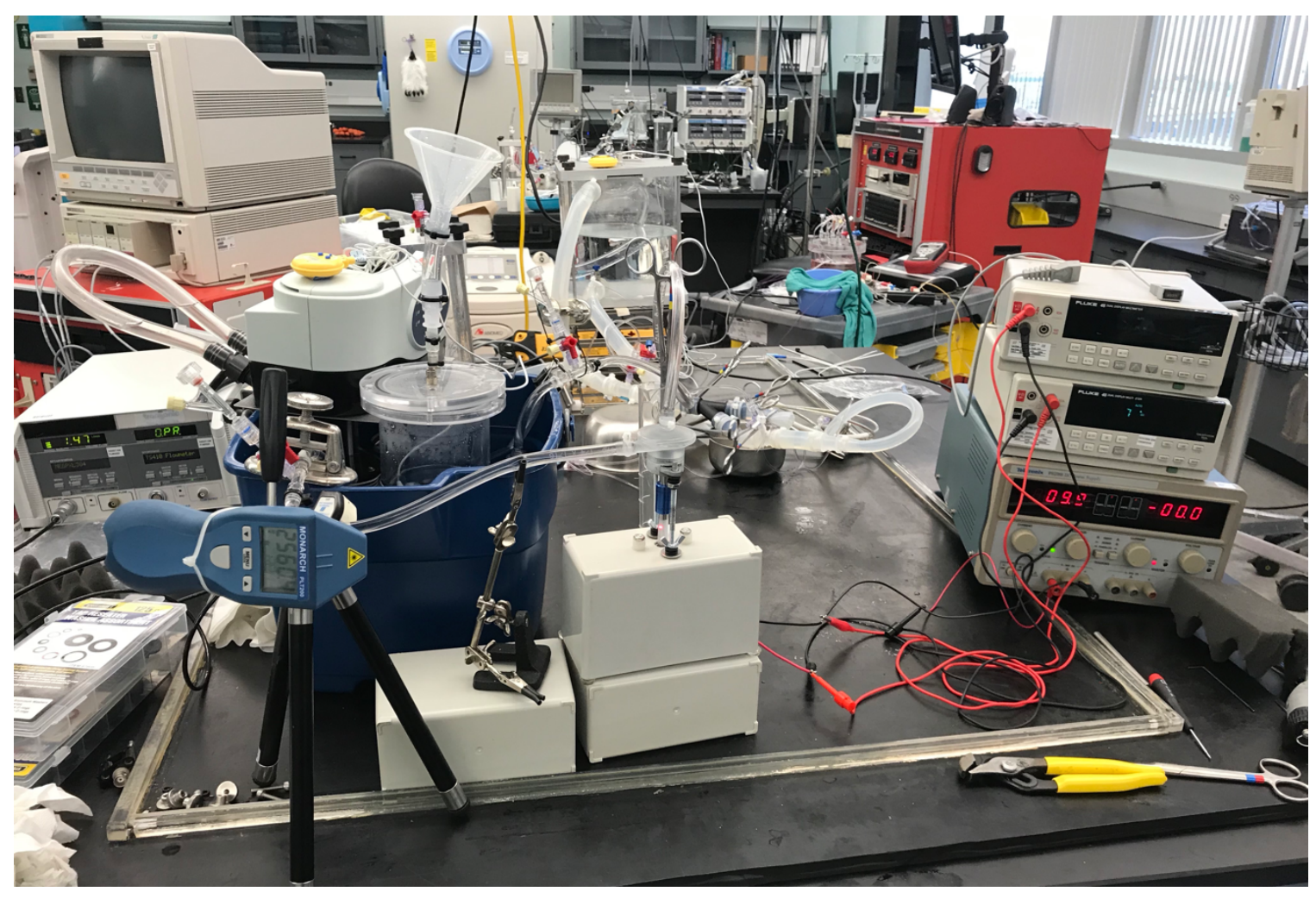




\section{APPENDIX D: VAD V2 ADDITIONAL SIMULATIONS}

\section{D.1 Additional VAD V2 CFD Simulations}

Additional CFD simulations than those found in Chapter 4 were conducted for VAD V2, comparing results to hydrodynamic performance and hydraulic torque values found empirically using the SFL model data. Six operational points (test conditions) of the pump were selected for rotational speeds of 1000, 2000, $3000,4000,5000$, and 6000 RPM at corresponding flow rates of $0.15,0.60,1.00$, $2.00,2.50$, and $3.50 \mathrm{~L} / \mathrm{min}$, respectively (Table D-1). Each of these test conditions were used to construct the computational fluid dynamics (CFD) model and perform simulations for further evaluation in SolidWorks Flow Simulation (SolidWorks 2019, Dassault Systèmes, Waltham, MA). The fluid properties of the previous analysis were adjusted to match the measured density and viscosity of the blood-analogue solution used in the SFL model. These simulations were conducted at each test condition to produce pump pressure $(\Delta \mathrm{P})$, impeller torque, and impeller forces (X, $\mathrm{Y}, \mathrm{Z}$ vectors) for each rotational speed and flow rate. In addition to the original Pediatric VAD geometry (CFD1), an additional geometry (CFD2) without the center opening through the rotor body was created (Figure D-1) to mimic the geometry of the empirical prototype (EXP1). This geometry was also simulated under all test conditions to compare the torques and forces of the impeller to those of the empirical prototype and the original 
Pediatric VAD geometry. An overview of SFL and CFD model experiments are presented in Figure D-2.

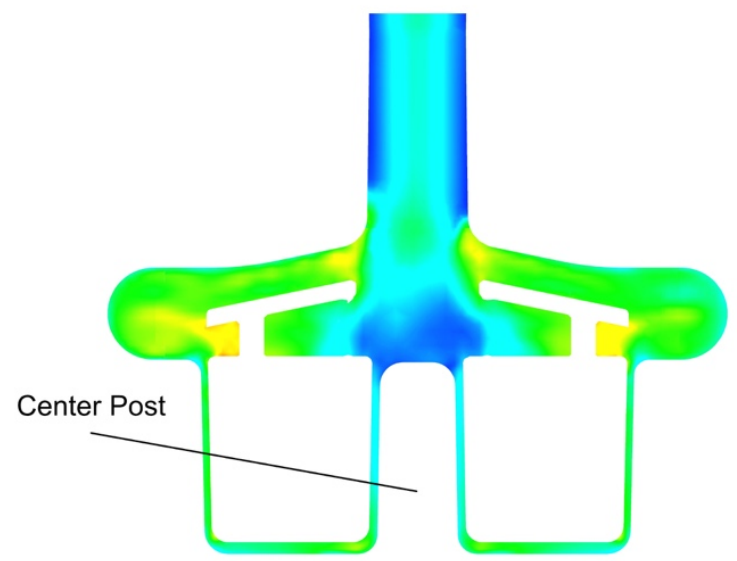

CFD1 Geometry

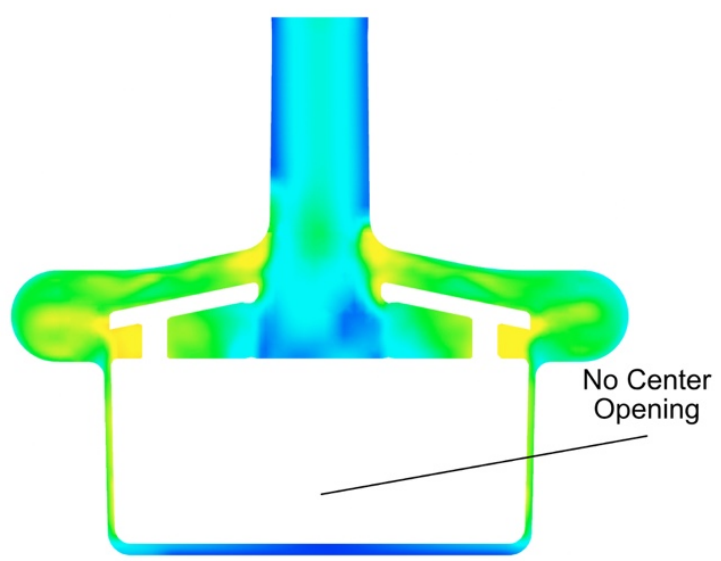

CFD2 Geometry

Figure D-1. Cut-views detailing the original, Inspired Pediatric VAD V2 geometry with rotor body center opening and lower pump housing center post used for CFD1 and of the modified VAD2 geometry without center opening used for CFD2 to mimic the geometry of the Inspired Pediatric VAD V2 shaft driven prototype (EXP1).

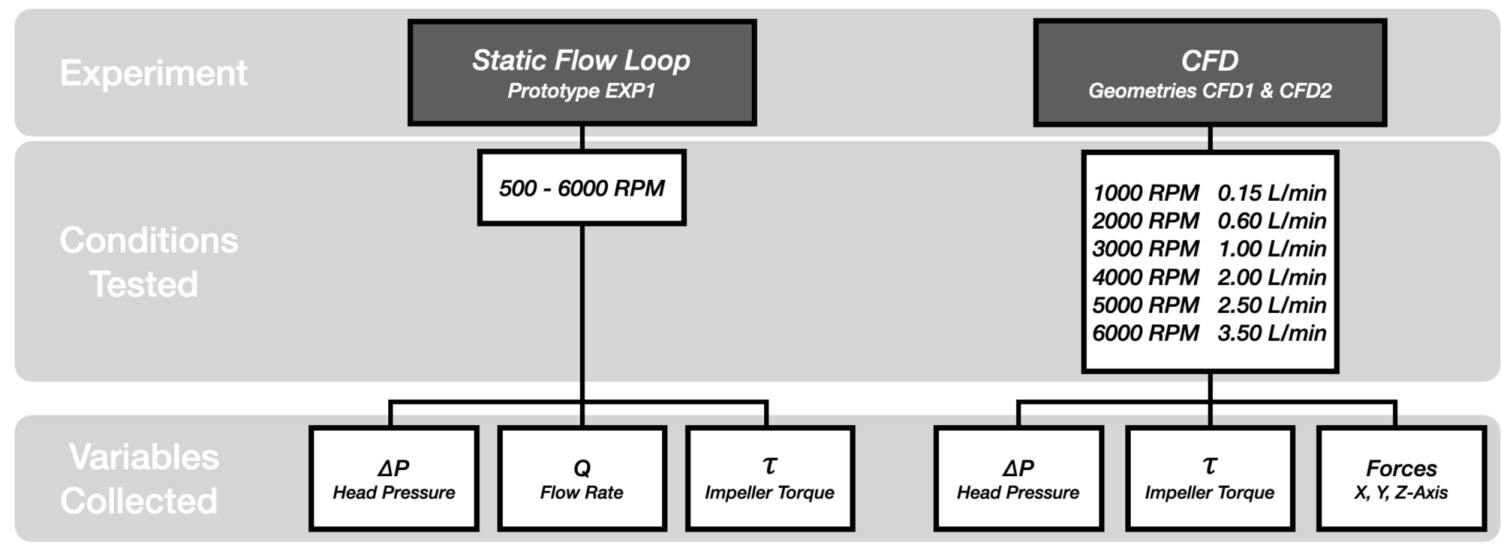

Figure D-2. A flow chart detailing the SFL (using the empirical pump prototype, EXP1) and CFD (using the pump geometries CFD1 and CFD2) experiments, the tested pump conditions (rotational speed or flow rate and rotational speed) for each experiment, and the collected variables for each experiment (pump pressure, flow rate, impeller torque and impeller $x, y$, and $z$ forces). 


\section{D.2 Empirical vs. CFD Results}

Results for the empirical cases $(1000,2000,3000,4000,5000$, and 6000

RPM and $0.15,0.60,1.00,2.00,2.50$, and $3.50 \mathrm{~L} / \mathrm{min}$ ) and CFD simulation results for pump geometries CFD1 and CFD2 are presented in Table D-1. CFD 1 and CFD2 simulation results were similar for all cases with less than a $2 \%$

Table D-1. Empirical results for the Inspired Pediatric VAD prototype in the SFL (EXP1) compared to CFD results for the two different impeller/rotor geometries (CFD1 and CFD2) including generated pressure head $(\Delta P)$ and impeller/rotor hydraulic torque ( $\boldsymbol{\tau}_{\text {Hydro) }}$.

\begin{tabular}{|c|c|c|c|c|c|c|c|c|}
\hline \multicolumn{9}{|c|}{ EXP1 - Empirical Prototype in Static Flow Loop } \\
\hline \multirow[b]{2}{*}{$\begin{array}{l}\text { Speed } \\
\text { (RPM) }\end{array}$} & \multirow[b]{2}{*}{$\begin{array}{l}\text { VAD Flow } \\
\text { (L/min) }\end{array}$} & \multirow[b]{2}{*}{$\begin{array}{c}\mathrm{P} 1 \\
(\mathrm{mmHg})\end{array}$} & \multirow[b]{2}{*}{$\begin{array}{c}\mathrm{P} 2 \\
(\mathrm{mmHg})\end{array}$} & \multirow[b]{2}{*}{$\begin{array}{c}\Delta \mathrm{P} \\
(\mathrm{mmHg})\end{array}$} & \multirow{2}{*}{$\begin{array}{c}\text { Hydraulic } \\
\text { Torque } \\
(\mathrm{mNm})\end{array}$} & \multicolumn{3}{|c|}{ Forces $(\mathrm{N})$} \\
\hline & & & & & & $\mathrm{x}$ & Y & Z \\
\hline 1000 & 0.15 & 7.63 & 0.37 & 7.26 & -0.33 & - & - & - \\
\hline 2000 & 0.60 & 24.41 & -2.60 & 27.01 & 0.08 & - & - & - \\
\hline 3000 & 1.00 & 58.73 & -7.65 & 66.38 & 2.57 & - & - & - \\
\hline 4000 & 2.00 & 55.86 & -31.23 & 87.09 & 8.65 & - & - & - \\
\hline 5000 & 2.50 & 119.47 & -44.85 & 164.32 & 11.43 & - & - & - \\
\hline 6000 & 3.50 & 123.43 & -87.34 & 210.77 & 15.56 & - & - & - \\
\hline \multicolumn{9}{|c|}{ CFD1 - Inspired VAD V2 } \\
\hline & & & & & $\begin{array}{c}\text { Hydraulic } \\
\text { Torque }\end{array}$ & & $\operatorname{rrces}(\wedge$ & \\
\hline $\begin{array}{l}\text { Speed } \\
\text { (RPM) }\end{array}$ & $\begin{array}{c}\text { VAD Flow } \\
(\text { L/min) }\end{array}$ & $\begin{array}{c}\mathrm{P} 1 \\
(\mathrm{mmHg})\end{array}$ & $\begin{array}{c}\mathrm{P} 2 \\
(\mathrm{mmHg})\end{array}$ & $\begin{array}{c}\Delta \mathrm{P} \\
(\mathrm{mmHg})\end{array}$ & & $x$ & $\mathrm{Y}$ & Z \\
\hline 1000 & 0.15 & 7.63 & -0.46 & 8.09 & 0.59 & 0.01 & 0.00 & 0.02 \\
\hline 2000 & 0.60 & 24.41 & -8.11 & 32.52 & 1.99 & 0.06 & 0.01 & 0.05 \\
\hline 3000 & 1.00 & 58.73 & -15.59 & 74.32 & 3.89 & 0.21 & -0.02 & 0.16 \\
\hline 4000 & 2.00 & 55.86 & -75.02 & 130.88 & 6.72 & -0.34 & 0.71 & 0.23 \\
\hline 5000 & 2.50 & 119.47 & -90.60 & 210.07 & 9.82 & 0.48 & 0.40 & 0.65 \\
\hline 6000 & 3.50 & 123.43 & -181.04 & 304.47 & 13.85 & 0.46 & 0.30 & 1.46 \\
\hline \multicolumn{9}{|c|}{ CFD2 - Inspired VAD V2 without Center Opening } \\
\hline & & & & & $\begin{array}{c}\text { Hydraulic } \\
\text { Torque }\end{array}$ & & $\operatorname{rrces}(\Lambda$ & \\
\hline $\begin{array}{l}\text { Speed } \\
\text { (RPM) }\end{array}$ & $\begin{array}{c}\text { VAD Flow } \\
(\text { L/min) }\end{array}$ & $\begin{array}{c}\mathrm{P} 1 \\
(\mathrm{mmHg})\end{array}$ & $\begin{array}{c}\mathrm{P} 2 \\
(\mathrm{mmHg})\end{array}$ & $\begin{array}{c}\Delta \mathrm{P} \\
(\mathrm{mmHg})\end{array}$ & & $x$ & Y & Z \\
\hline 1000 & 0.15 & 7.63 & -0.43 & 8.06 & 0.59 & 0.01 & 0.00 & 0.15 \\
\hline 2000 & 0.60 & 24.41 & -7.62 & 32.03 & 1.95 & 0.07 & 0.01 & 0.68 \\
\hline 3000 & 1.00 & 58.73 & -15.72 & 74.45 & 3.78 & 0.15 & 0.04 & 1.49 \\
\hline 4000 & 2.00 & 55.86 & -76.08 & 131.94 & 6.52 & 0.29 & 0.11 & 2.71 \\
\hline 5000 & 2.50 & 119.47 & -91.65 & 211.12 & 9.45 & 0.40 & 0.16 & 4.12 \\
\hline 6000 & 3.50 & 123.43 & -180.70 & 304.13 & 13.46 & 0.54 & 0.26 & 5.96 \\
\hline
\end{tabular}


difference for all generated $\Delta \mathrm{P}$ values and less than a $4 \%$ difference for all generated $\boldsymbol{\tau}_{\text {Hydro }}$ values. CFD simulations produced varied results compared to the empirical prototype (EXP1) for both compared values (Figures D-3 and D-4). CFD results for $\boldsymbol{\tau}_{\text {Hydro }}$ were higher at lower rotational speeds than EXP1 with a difference of $155 \%$ for the $1000 \mathrm{RPM}$ case. Conversely, CFD generated $\triangle \mathrm{P}$ values showed less difference at lower rotational speeds (1000 to 3000 RPM, $10 \%$ to $17 \%$ difference in $\Delta P$ ) with the difference in $\Delta P$ value increasing as rotational speed increased (up to a $33 \%$ difference).

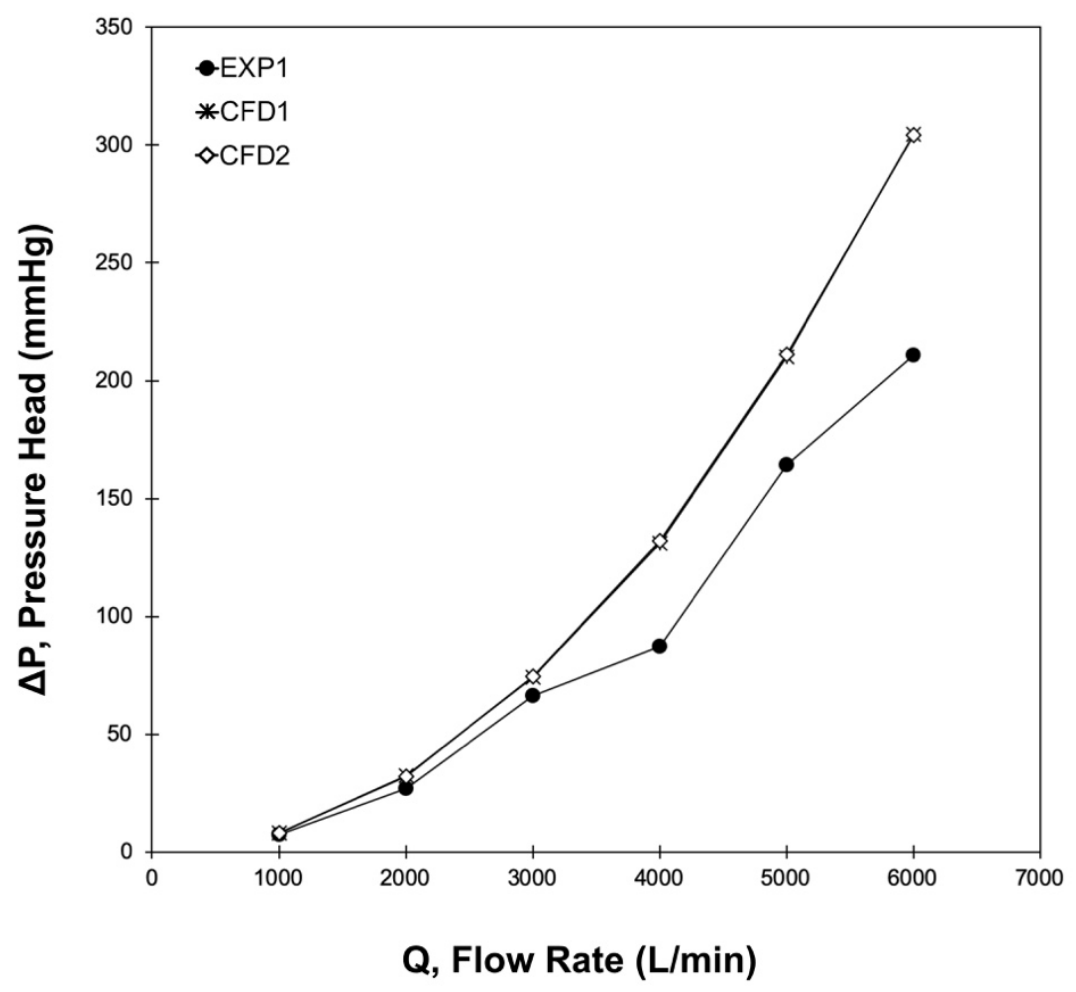

Figure D-3. Pressure vs. flow rate values for the Inspired Pediatric VAD V2 prototype compared with CFD simulation results CFD1 and CFD 2 for rotational speeds 500 to 6000 RPM 


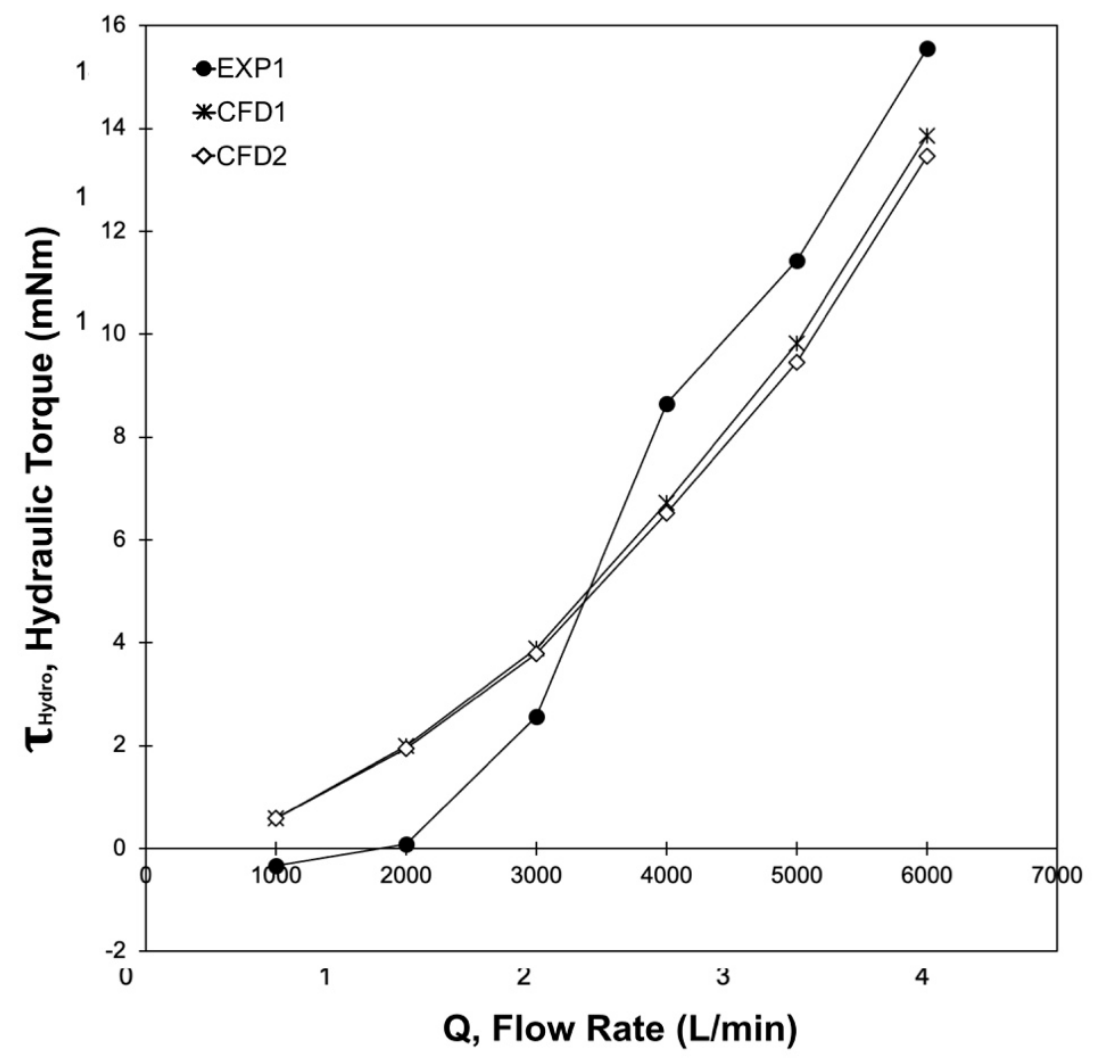

Figure D-4. Hydraulic torque values for the Inspired Pediatric VAD V2 prototype compared with CFD simulation results CFD 1 and CFD 2 for rotational speeds 500 to 6000 RPM 


\section{D.3 Axial Impeller Force}

Tangential and radial forces ( $X$ and $Y$ vectors) were relatively small $(0.5 \mathrm{~N}$ or less) for CFD1 and CFD2 for all rotational speeds. The axial force (Z vector) in the positive direction increased with raising the rotational speed, likely due to the lift force created by the impeller rotation, for CFD1 and CFD2. CFD2 (solid impeller/rotor) generated much higher axial forces (5.96 N at $6000 \mathrm{RPM})$ than CFD1 (impeller/rotor with center opening) (1.46 N at $6000 \mathrm{RPM}$ ) likely due to the additional secondary flow path around the impeller/rotor added as a design feature to help negate generated lift forces, as illustrated in Figure D-5. These results confirmed the previous decision to include the center opening in the rotor design.

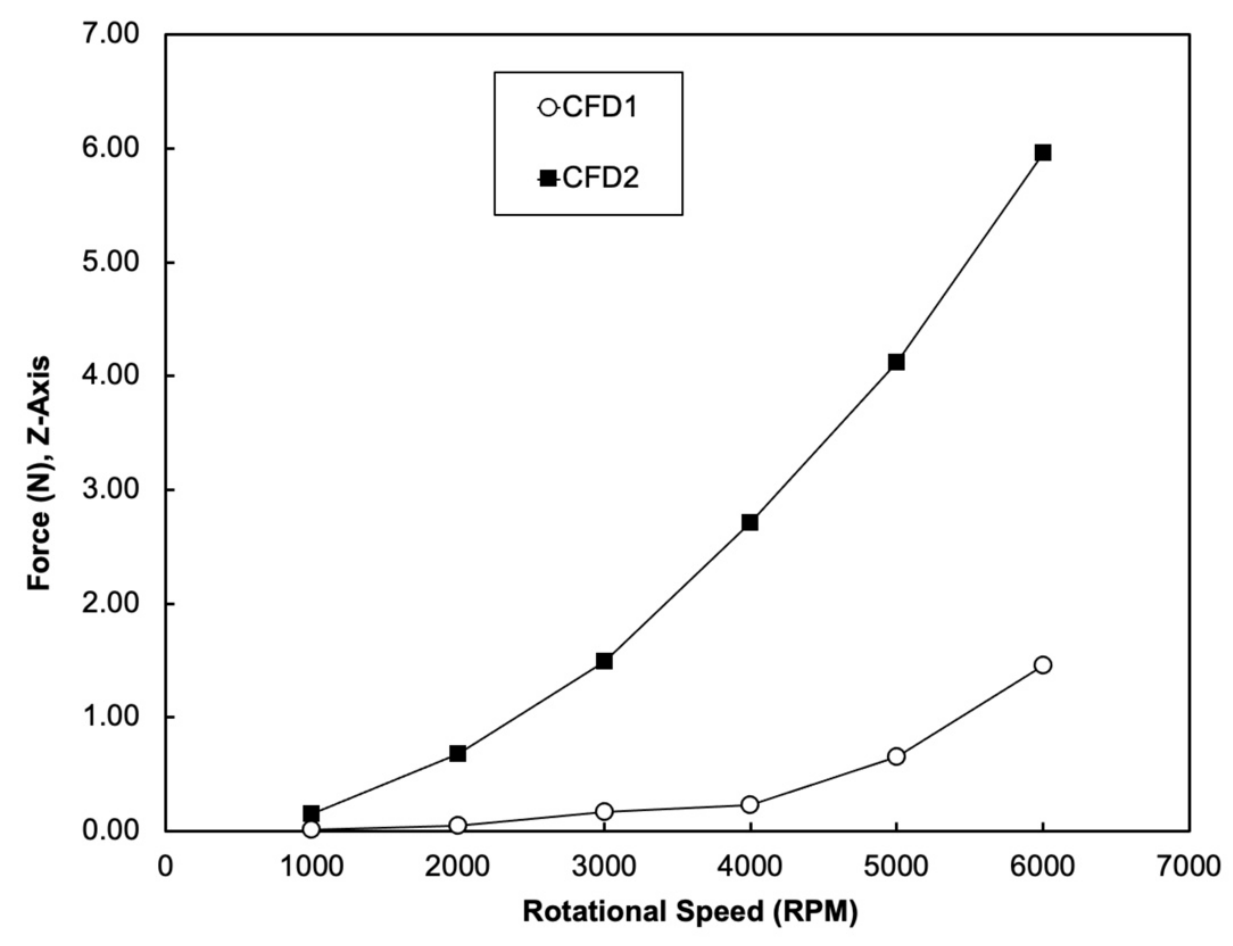

Figure D-5. Impeller/rotor force in the axial (Z-axis) direction relative to rotational speed for geometries CFD1 and CFD2. 


\section{APPENDIX E: CHAPTER 5 SUPPLEMENT}

Table E-1. Summary of Reynolds number calculations for Inspired Pediatric $V A D$ V3 pump inlet and impeller for flow rates (Q) $0.50-4.00 \mathrm{~L} / \mathrm{min}$ and rotational speeds (n) 3000, 4000, and 5000 RPM.

\begin{tabular}{|c|c|c|c|c|c|}
\hline \multicolumn{5}{|c|}{ VAD Impeller - Flow across a Rotating Disk } & \\
\hline $\begin{array}{l}\text { Rotational } \\
\text { Speed, } n \\
\text { (RPM) }\end{array}$ & $\begin{array}{c}\text { Impeller } \\
\text { Radius, } r(\mathrm{~mm})\end{array}$ & Formula & $\operatorname{Re}$ & $\begin{array}{c}\text { Critical } \\
\text { Transition } \\
\text { Re }\end{array}$ & \\
\hline 3000 & \multirow{3}{*}{16.1} & \multirow{3}{*}{$R e=\frac{2 r^{2} \rho n}{\mu}$} & 49325 & \multirow{3}{*}{$10^{\wedge} 6$} & \\
\hline 4000 & & & 65767 & & \\
\hline 5000 & & & 82209 & & \\
\hline \multicolumn{6}{|c|}{ VAD Inlet - Flow through a Circular Pipe } \\
\hline $\begin{array}{l}\text { Flow Rate, } Q \\
\text { (L/min) }\end{array}$ & $\begin{array}{l}\text { Inlet Velocity, } \\
\qquad(\mathrm{m} / \mathrm{s})\end{array}$ & $\begin{array}{l}\text { Inlet Diameter, } \\
D(\mathrm{~mm})\end{array}$ & Formula & $\operatorname{Re}$ & $\begin{array}{c}\text { Critical } \\
\text { Transition Re }\end{array}$ \\
\hline 0.50 & 0.26733 & \multirow{6}{*}{6.35} & \multirow{6}{*}{$R e=\frac{\rho V D}{\mu}$} & 514 & \multirow{6}{*}{2300} \\
\hline 1.00 & 0.53466 & & & 1028 & \\
\hline 2.00 & 1.0693 & & & 2056 & \\
\hline 2.50 & 1.3367 & & & 2571 & \\
\hline 3.50 & 1.8713 & & & 3599 & \\
\hline 4.00 & 2.1051 & & & 4048 & \\
\hline
\end{tabular}




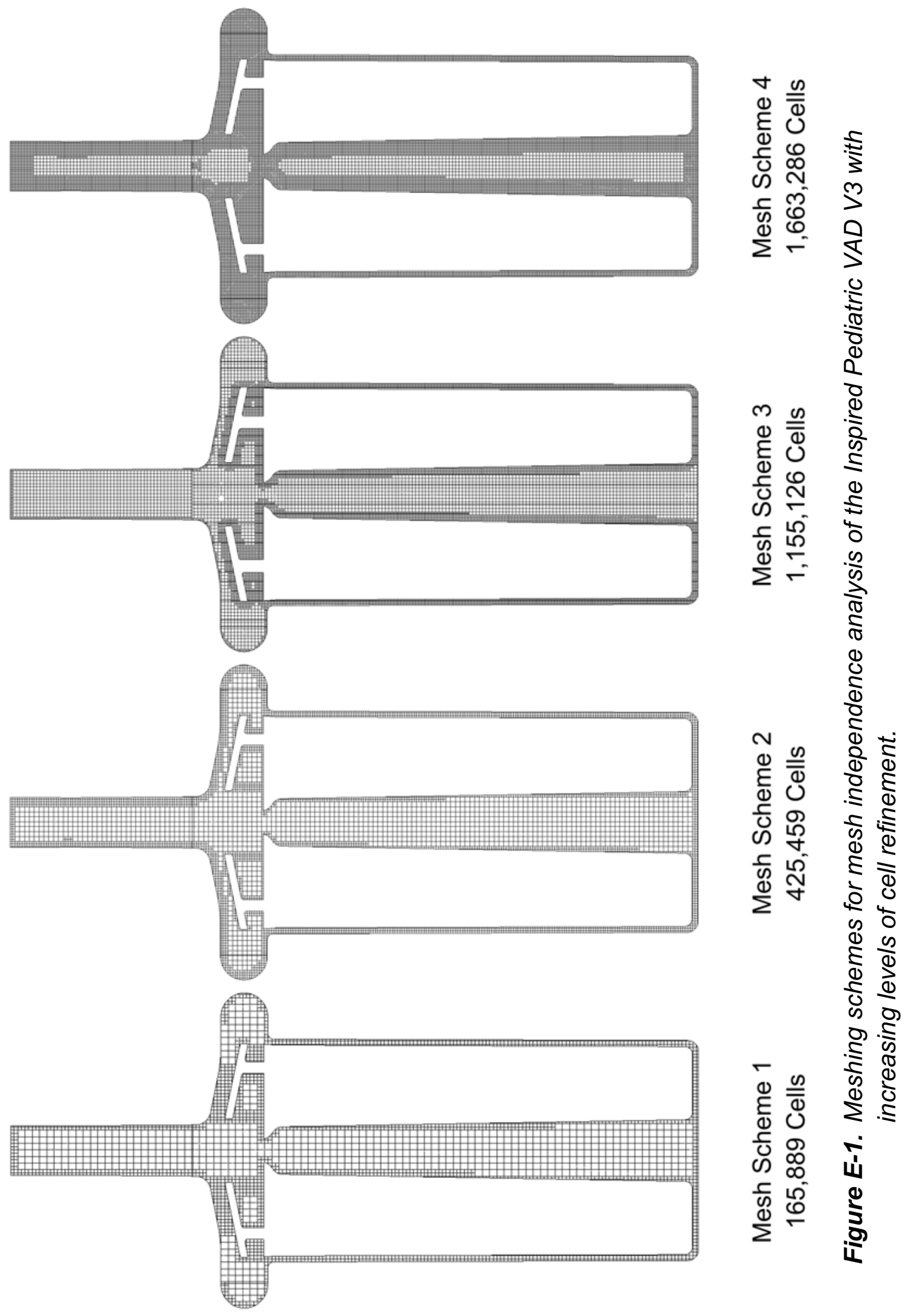




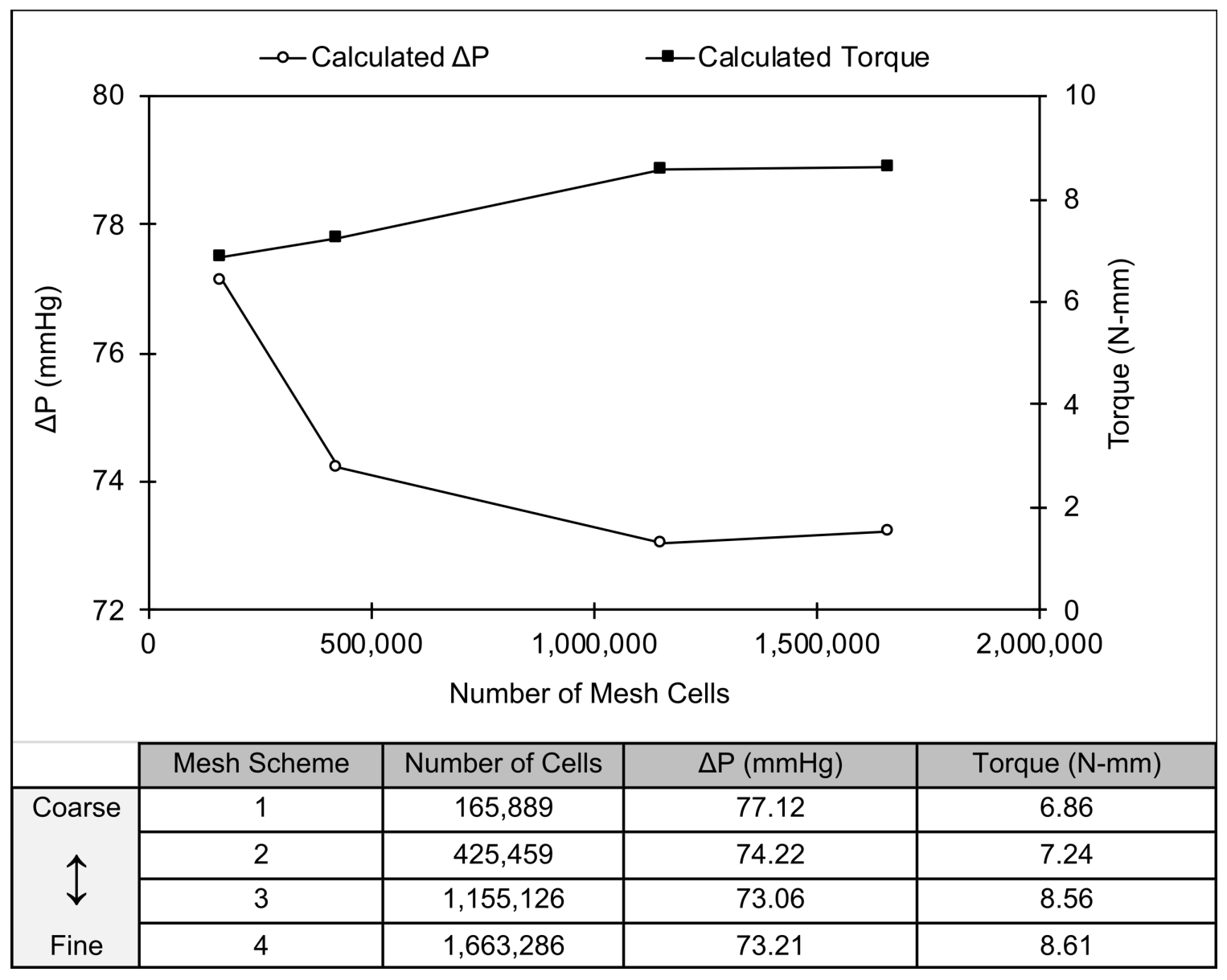

Figure E-2. Generated pressure $(\Delta P)$ and torque values for meshing schemes 1 - 4 at 3000 RPM and $1.00 \mathrm{~L} / \mathrm{min}$. Meshing Scheme 3 showed less than a $0.50 \%$ difference in converged $\triangle P$ value and less than a $1 \%$ difference in converged torque value compared to the finest meshing Scheme 4. 
Table E-2. Summary of computational fluid dynamics (CFD) predictions of head pressure, impeller hydraulic torque, and impeller translational forces for Inspired Pediatric VAD V2 at rotational speeds 3000, 4000, and 5000 RPM and various flow rates.

\begin{tabular}{|c|c|c|c|c|c|c|c|c|}
\hline \multicolumn{9}{|c|}{ Inspired Pediatric VAD V2 - CFD - 3000 RPM } \\
\hline \multirow{2}{*}{$\begin{array}{l}\text { Speed } \\
\text { (RPM) }\end{array}$} & \multirow{2}{*}{$\begin{array}{l}\text { VAD Flow } \\
\text { (L/min) }\end{array}$} & \multirow{2}{*}{$\begin{array}{c}\mathrm{P} 1 \\
(\mathrm{mmHg})\end{array}$} & \multirow{2}{*}{$\begin{array}{c}\mathrm{P} 2 \\
(\mathrm{mmHg})\end{array}$} & \multirow{2}{*}{$\begin{array}{c}\text { Head } \\
(\mathrm{mmHg})\end{array}$} & \multirow{2}{*}{$\begin{array}{c}\text { Hydraulic } \\
\text { Torque } \\
(\mathrm{mNm})\end{array}$} & \multicolumn{3}{|c|}{ Forces $(\mathrm{N})$} \\
\hline & & & & & & $x$ & Y & Z \\
\hline 3000 & 2.50 & 100.00 & 41.38 & 58.62 & 4.43 & 0.10 & 0.24 & 0.62 \\
\hline 3000 & 1.50 & 100.00 & 32.27 & 67.73 & 4.03 & 0.07 & 0.22 & 0.28 \\
\hline 3000 & 1.00 & 100.00 & 28.90 & 71.10 & 3.67 & 0.11 & 0.11 & 0.12 \\
\hline 3000 & 0.50 & 100.00 & 27.48 & 72.52 & 3.46 & 0.07 & 0.02 & 0.01 \\
\hline \multicolumn{9}{|c|}{ Inspired Pediatric VAD V2 - CFD - 4000 RPM } \\
\hline \multirow{2}{*}{$\begin{array}{l}\text { Speed } \\
\text { (RPM) }\end{array}$} & \multirow{2}{*}{$\begin{array}{l}\text { VAD Flow } \\
\text { (L/min) }\end{array}$} & \multirow{2}{*}{$\begin{array}{c}\mathrm{P} 1 \\
(\mathrm{mmHg})\end{array}$} & \multirow{2}{*}{$\begin{array}{c}\mathrm{P} 2 \\
(\mathrm{mmHg})\end{array}$} & \multirow{2}{*}{$\begin{array}{c}\text { Head } \\
(\mathrm{mmHg})\end{array}$} & \multirow{2}{*}{$\begin{array}{c}\text { Hydraulic } \\
\text { Torque } \\
(\mathrm{mNm})\end{array}$} & \multicolumn{3}{|c|}{ Forces $(\mathrm{N})$} \\
\hline & & & & & & $x$ & $Y$ & Z \\
\hline 4000 & 3.00 & 100.00 & -16.28 & 116.28 & 7.02 & 0.18 & 0.39 & 0.96 \\
\hline 4000 & 2.00 & 100.00 & -26.30 & 126.30 & 6.36 & 0.19 & 0.24 & 0.53 \\
\hline 4000 & 1.00 & 100.00 & -31.10 & 131.10 & 5.95 & 0.08 & 0.16 & 0.27 \\
\hline 4000 & 0.50 & 100.00 & -40.77 & 140.77 & 5.63 & 0.02 & 0.11 & 0.07 \\
\hline \multicolumn{9}{|c|}{ Inspired Pediatric VAD V2 - CFD - 5000 RPM } \\
\hline \multirow{2}{*}{$\begin{array}{l}\text { Speed } \\
\text { (RPM) }\end{array}$} & \multirow{2}{*}{$\begin{array}{l}\text { VAD Flow } \\
\text { (L/min) }\end{array}$} & \multirow{2}{*}{$\begin{array}{c}\mathrm{P} 1 \\
(\mathrm{mmHg})\end{array}$} & \multirow{2}{*}{$\begin{array}{c}\mathrm{P} 2 \\
(\mathrm{mmHg})\end{array}$} & \multirow{2}{*}{$\begin{array}{c}\text { Head } \\
(\mathrm{mmHg})\end{array}$} & \multirow{2}{*}{$\begin{array}{c}\text { Hydraulic } \\
\text { Torque } \\
(\mathrm{mNm})\end{array}$} & \multicolumn{3}{|c|}{ Forces $(\mathrm{N})$} \\
\hline & & & & & & $x$ & $Y$ & $z$ \\
\hline 5000 & 4.00 & 100.00 & -80.90 & 180.90 & 10.52 & 0.36 & 0.50 & 1.61 \\
\hline 5000 & 3.00 & 100.00 & -99.17 & 199.17 & 9.82 & -0.70 & 0.63 & 1.22 \\
\hline 5000 & 2.00 & 100.00 & -102.91 & 202.91 & 8.96 & 0.15 & 0.13 & 0.46 \\
\hline 5000 & 1.00 & 100.00 & -117.80 & 217.80 & 7.96 & 0.16 & 0.11 & 0.30 \\
\hline 5000 & 0.50 & 100.00 & -124.85 & 224.85 & 7.70 & 0.00 & 0.47 & 0.31 \\
\hline
\end{tabular}


Table E-3. Summary of computational fluid dynamics (CFD) predictions of head pressure, impeller hydraulic torque, and impeller translational forces for Inspired Pediatric VAD V3 at rotational speeds 3000, 4000, and 5000 RPM and various flow rates.

\begin{tabular}{|c|c|c|c|c|c|c|c|c|}
\hline \multicolumn{9}{|c|}{ Inspired Pediatric VAD V3 - CFD - 3000 RPM } \\
\hline \multirow{2}{*}{$\begin{array}{l}\text { Speed } \\
\text { (RPM) }\end{array}$} & \multirow{2}{*}{$\begin{array}{l}\text { VAD Flow } \\
\text { (L/min) }\end{array}$} & \multirow{2}{*}{$\begin{array}{c}\mathrm{P} 1 \\
(\mathrm{mmHg})\end{array}$} & \multirow{2}{*}{$\begin{array}{c}\mathrm{P} 2 \\
(\mathrm{mmHg})\end{array}$} & \multirow{2}{*}{$\begin{array}{c}\text { Head } \\
(\mathrm{mmHg})\end{array}$} & \multirow{2}{*}{$\begin{array}{c}\text { Hydraulic } \\
\text { Torque } \\
(\mathrm{mNm})\end{array}$} & \multicolumn{3}{|c|}{ Forces $(\mathrm{N})$} \\
\hline & & & & & & $x$ & Y & Z \\
\hline 3000 & 2.50 & 140.62 & 200.00 & 59.38 & 9.37 & 0.12 & 0.41 & 0.83 \\
\hline 3000 & 1.00 & -14.71 & 58.73 & 73.44 & 8.56 & 0.01 & 0.20 & 0.13 \\
\hline 3000 & 0.50 & 125.50 & 200.00 & 74.50 & 8.39 & 0.07 & 0.20 & -0.02 \\
\hline \multicolumn{9}{|c|}{ Inspired Pediatric VAD V3 - CFD - 4000 RPM } \\
\hline \multirow{2}{*}{$\begin{array}{l}\text { Speed } \\
\text { (RPM) }\end{array}$} & \multirow{2}{*}{$\begin{array}{l}\text { VAD Flow } \\
\text { (L/min) }\end{array}$} & \multirow{2}{*}{$\begin{array}{c}\mathrm{P} 1 \\
(\mathrm{mmHg})\end{array}$} & \multirow{2}{*}{$\begin{array}{c}\mathrm{P} 2 \\
(\mathrm{mmHg})\end{array}$} & \multirow{2}{*}{$\begin{array}{c}\text { Head } \\
(\mathrm{mmHg})\end{array}$} & \multirow{2}{*}{$\begin{array}{c}\text { Hydraulic } \\
\text { Torque } \\
(\mathrm{mNm})\end{array}$} & \multicolumn{3}{|c|}{ Forces $(\mathrm{N})$} \\
\hline & & & & & & $\mathrm{x}$ & $\mathrm{Y}$ & Z \\
\hline 4000 & 3.00 & 85.31 & 200.00 & 114.69 & 14.35 & 0.14 & 0.73 & 1.30 \\
\hline 4000 & 2.00 & -68.97 & 55.86 & 124.83 & 13.69 & 0.11 & 0.60 & 0.45 \\
\hline 4000 & 1.00 & 67.57 & 200.00 & 132.43 & 13.10 & 0.10 & 0.40 & 0.35 \\
\hline \multicolumn{9}{|c|}{ Inspired Pediatric VAD V3 - CFD - 5000 RPM } \\
\hline \multirow{2}{*}{$\begin{array}{l}\text { Speed } \\
\text { (RPM) }\end{array}$} & \multirow{2}{*}{$\begin{array}{l}\text { VAD Flow } \\
\text { (L/min) }\end{array}$} & \multirow{2}{*}{$\begin{array}{c}\mathrm{P} 1 \\
(\mathrm{mmHg})\end{array}$} & \multirow{2}{*}{$\begin{array}{c}\mathrm{P} 2 \\
(\mathrm{mmHg})\end{array}$} & \multirow{2}{*}{$\begin{array}{c}\text { Head } \\
(\mathrm{mmHg})\end{array}$} & \multirow{2}{*}{$\begin{array}{c}\text { Hydraulic } \\
\text { Torque } \\
(\mathrm{mNm})\end{array}$} & \multicolumn{3}{|c|}{ Forces $(\mathrm{N})$} \\
\hline & & & & & & $x$ & $\mathrm{Y}$ & Z \\
\hline 5000 & 4.00 & 21.06 & 200.00 & 178.94 & 20.36 & 178.94 & 21.06 & 200.00 \\
\hline 5000 & 3.50 & 13.47 & 200.00 & 186.53 & 19.89 & 186.53 & 13.47 & 200.00 \\
\hline 5000 & 2.50 & -80.81 & 119.47 & 200.28 & 19.62 & 200.28 & -80.81 & 119.47 \\
\hline 5000 & 1.00 & -10.82 & 200.00 & 210.82 & 18.07 & 210.82 & -10.82 & 200.00 \\
\hline
\end{tabular}

Table E-4. Summary of data for additional CFD simulation cases for Inspired Pediatric VAD V3 at rotational speeds 1000, 2000, and 6000 RPM.

\begin{tabular}{|c|c|c|c|c|c|c|c|c|}
\hline \multirow{3}{*}{$\begin{array}{l}\text { Speed } \\
\text { (RPM) }\end{array}$} & \multicolumn{8}{|c|}{ Inspired Pediatric VAD V3 - Laminar, Non-Newtonian, 37 C } \\
\hline & \multirow{2}{*}{$\begin{array}{l}\text { VAD Flow } \\
\text { (L/min) }\end{array}$} & \multirow{2}{*}{$\begin{array}{c}\mathrm{P} 1 \\
(\mathrm{mmHg})\end{array}$} & \multirow{2}{*}{$\begin{array}{c}\mathrm{P} 2 \\
(\mathrm{mmHg})\end{array}$} & \multirow{2}{*}{$\begin{array}{c}\text { Head } \\
(\mathrm{mmHg})\end{array}$} & \multirow{2}{*}{$\begin{array}{l}\text { Hydraulic } \\
\text { Torque } \\
(\mathrm{mNm})\end{array}$} & \multicolumn{3}{|c|}{ Forces $(\mathrm{N})$} \\
\hline & & & & & & $x$ & $Y$ & Z \\
\hline 1000 & 0.15 & -0.11 & 7.63 & 7.74 & 1.20 & 0.01 & 0.03 & -0.09 \\
\hline 2000 & 0.60 & -8.44 & 24.41 & 32.85 & 4.35 & 0.00 & 0.04 & -0.08 \\
\hline 6000 & 3.50 & -171.66 & 123.43 & 295.09 & 25.85 & 0.11 & 1.14 & 2.15 \\
\hline
\end{tabular}




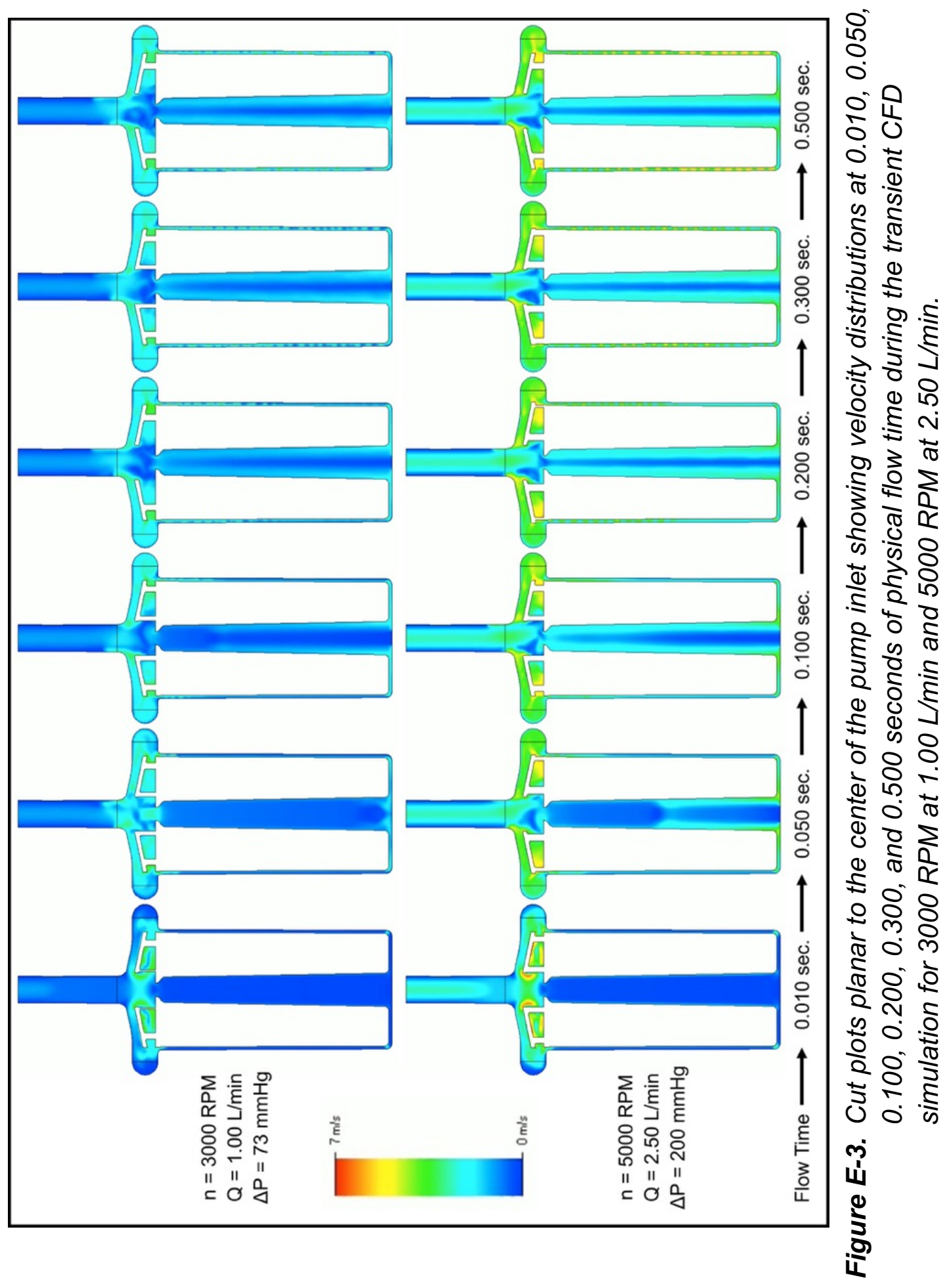




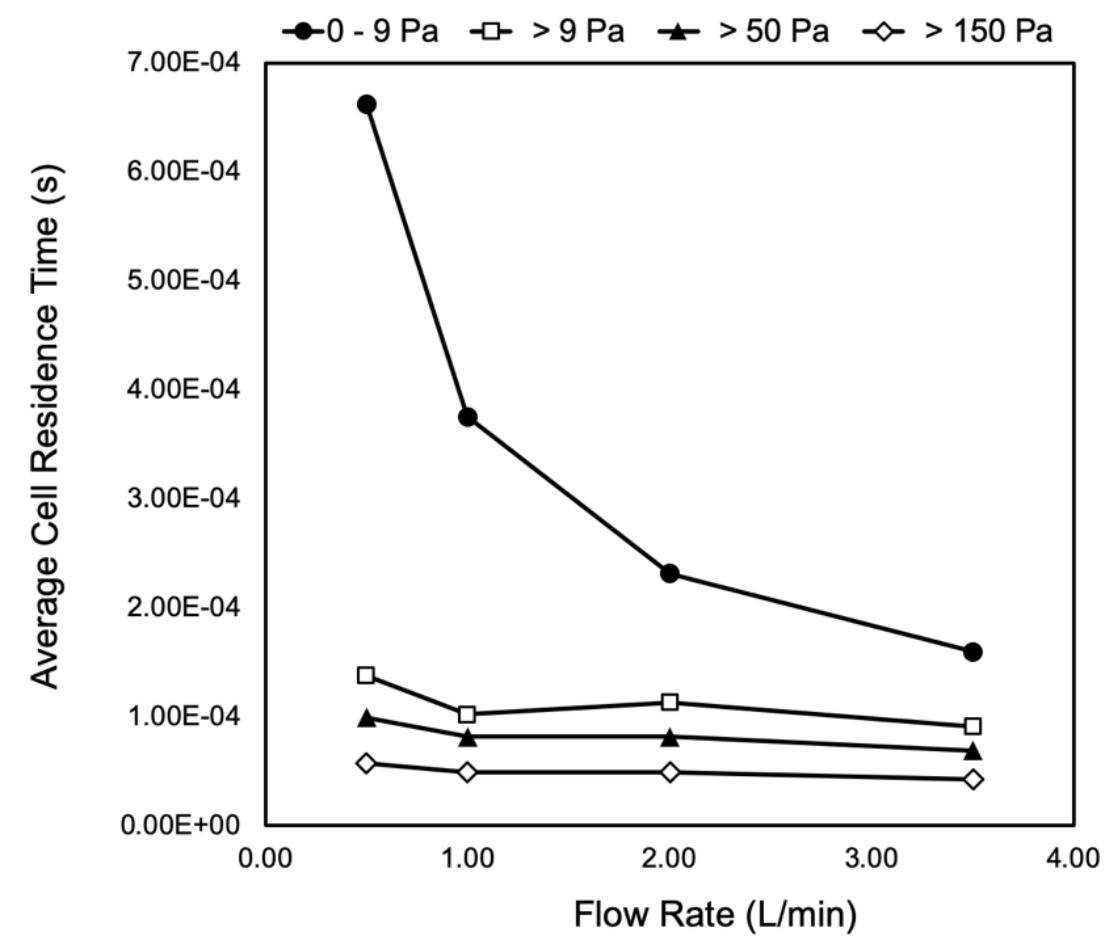

Figure E-4. Average mesh cell residence times at critical shear stress thresholds of $9 \mathrm{~Pa}$ (vWF damage), $50 \mathrm{~Pa}$ (platelet activation), and $150 \mathrm{~Pa}$ (hemolysis) for flow rates $0.50,1.00,2.00$, and $3.50 \mathrm{~L} / \mathrm{min}$. 


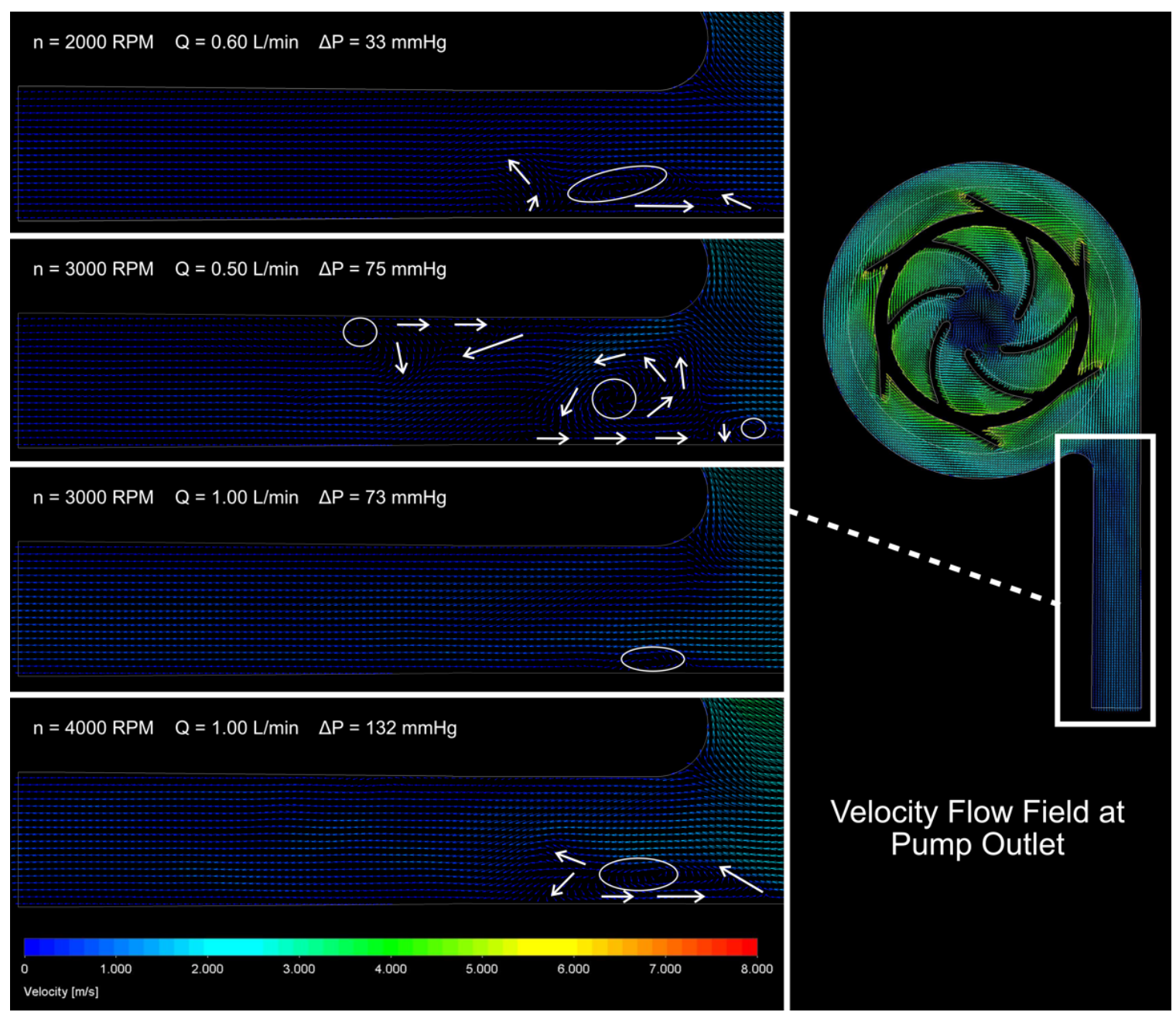

Figure E-5. Velocity flow field vectors at the Inspired Pediatric VAD V3 pump outlet. At low flows of $1.00 \mathrm{~L} / \mathrm{min}$ or lower, select areas of flow recirculation and retrograde occur. 

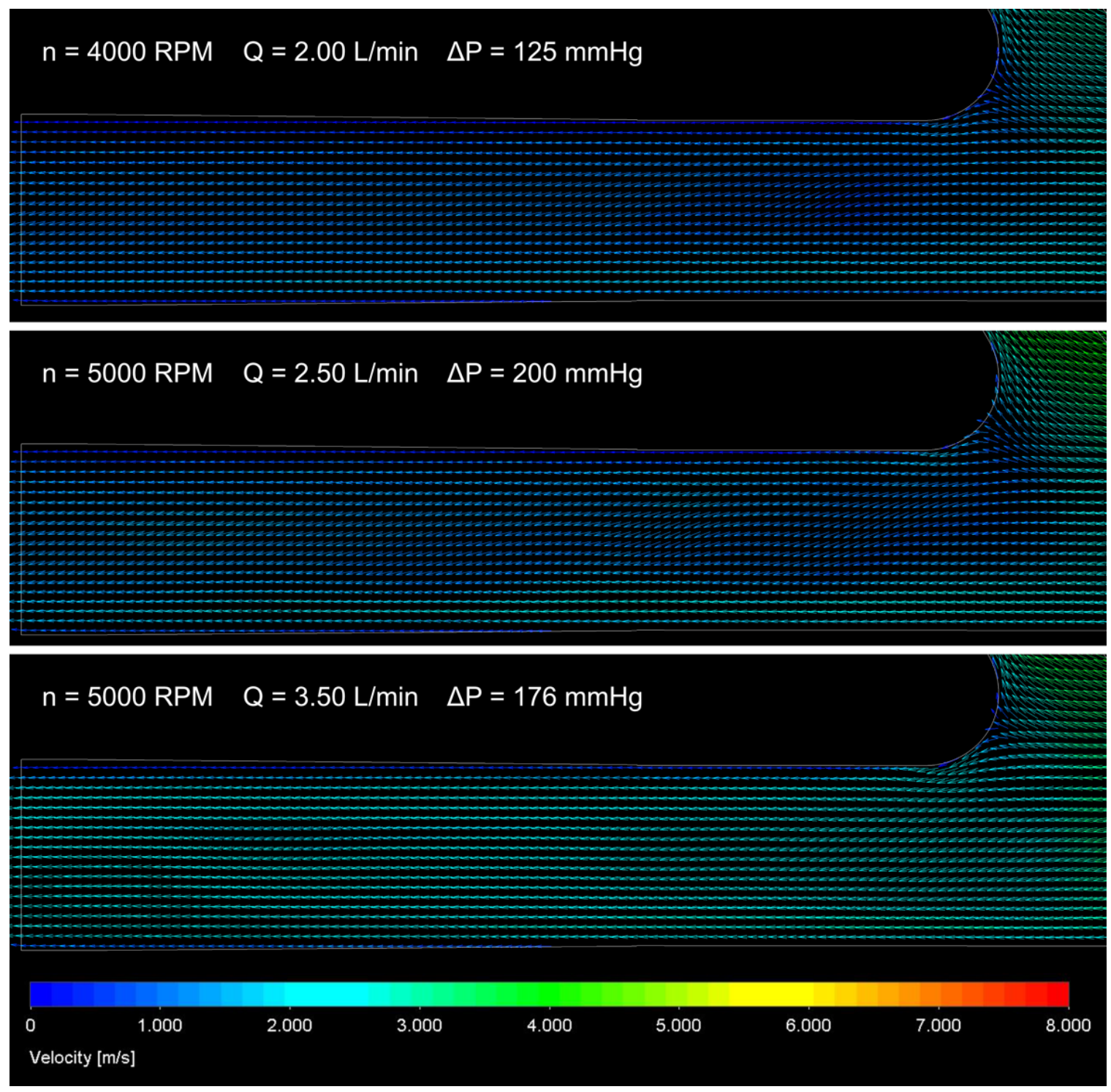

Figure E-6. Velocity flow field vectors at the Inspired Pediatric VAD V3 pump outlet. At higher flows above $1.00 \mathrm{~L} / \mathrm{min}$, all flow vectors travel toward the pump outlet eliminating stagnation and recirculation zones that are present at lower flows. 


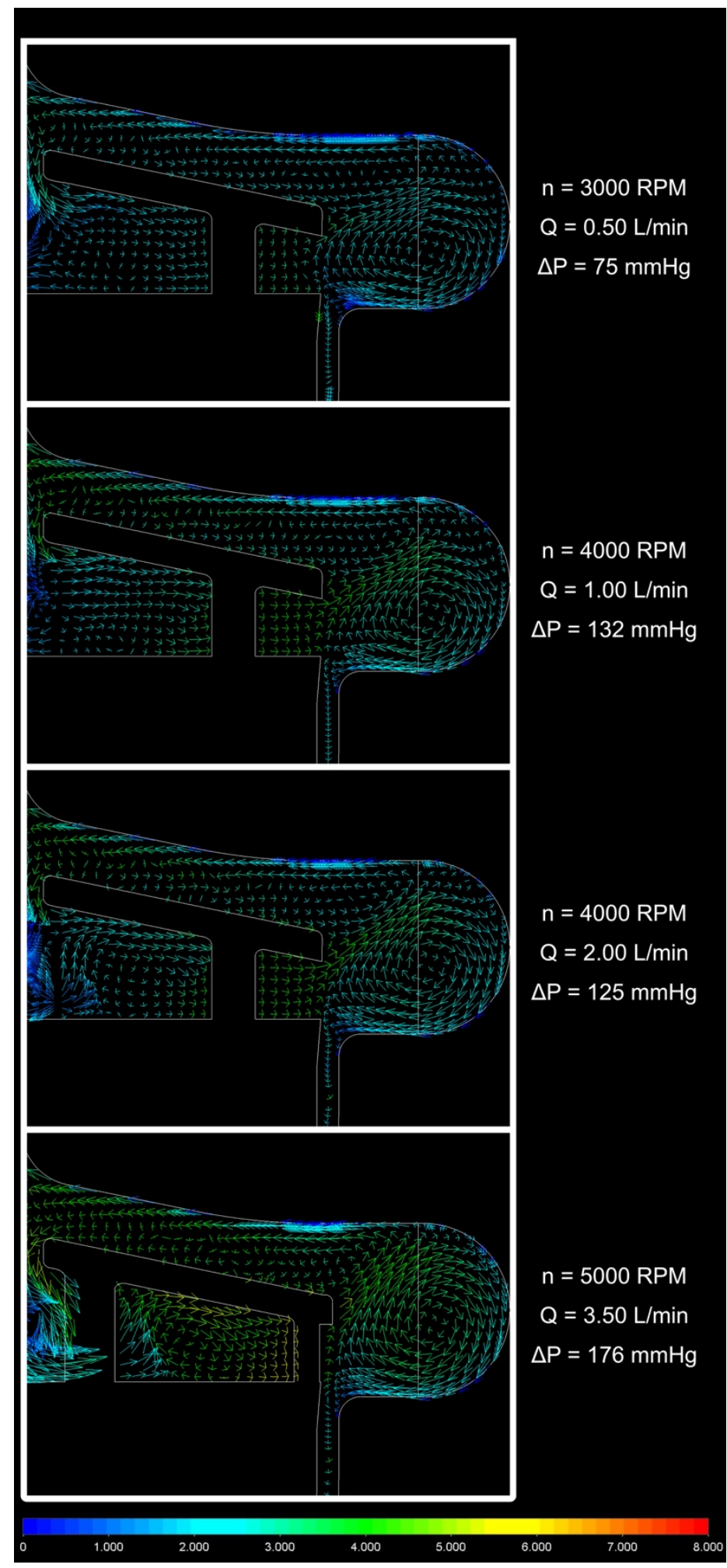

Figure E-7. Velocity flow field vectors inside the Inspired Pediatric VAD V3 volute at four flow and pressure conditions. 


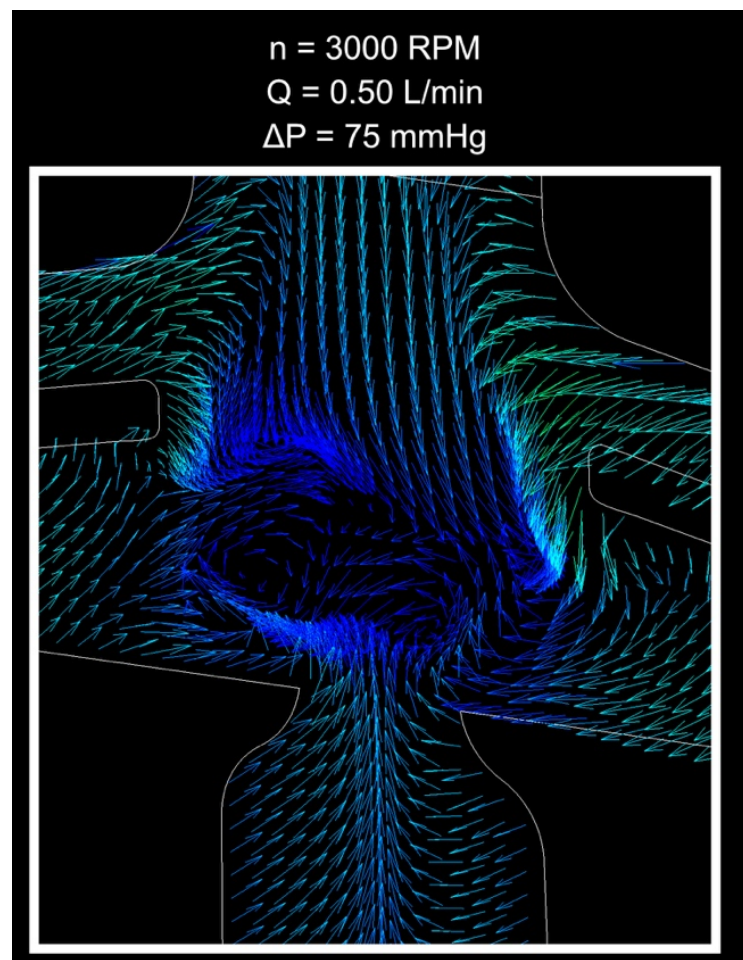

$\mathrm{n}=4000 \mathrm{RPM}$

$\mathrm{Q}=2.00 \mathrm{~L} / \mathrm{min}$

$\Delta \mathrm{P}=125 \mathrm{mmHg}$

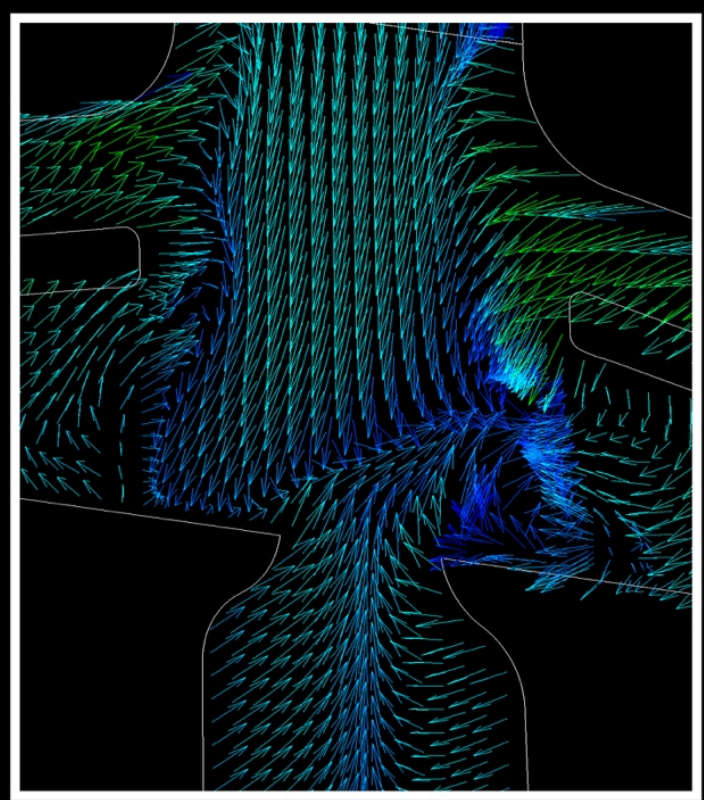

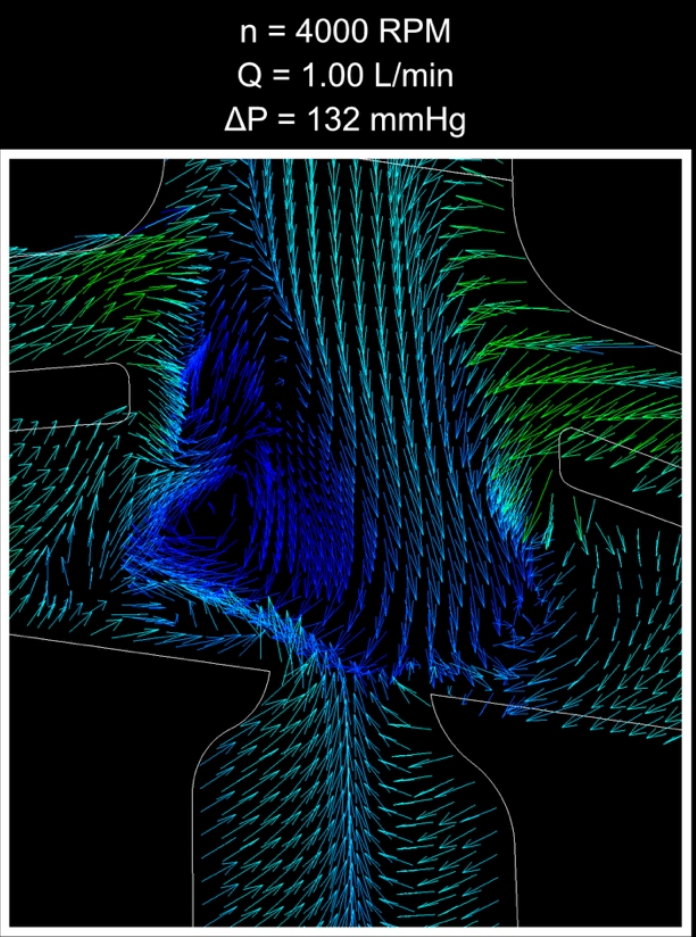

$\mathrm{n}=5000 \mathrm{RPM}$

$\mathrm{Q}=3.50 \mathrm{~L} / \mathrm{min}$

$\Delta \mathrm{P}=176 \mathrm{mmHg}$

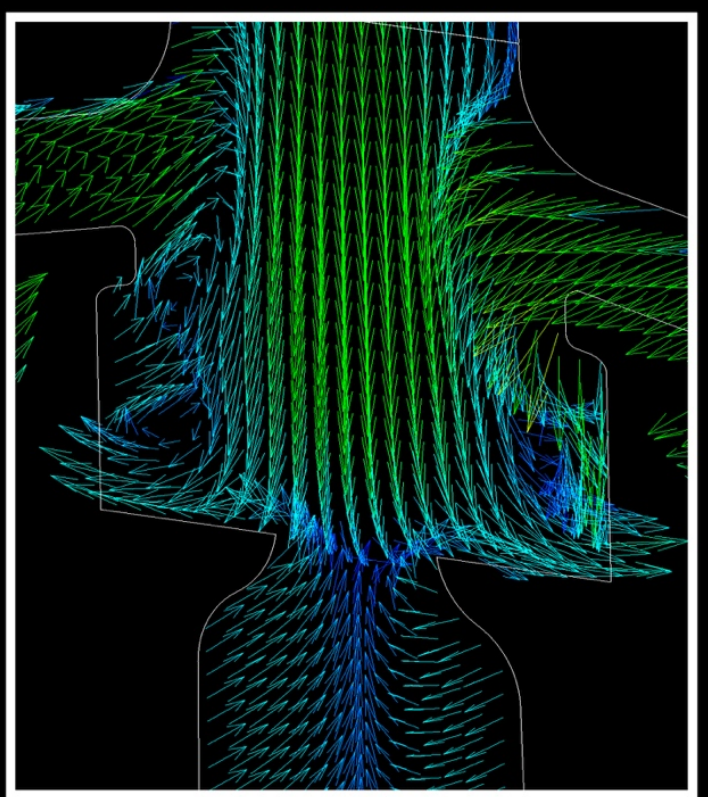

5.000

6.000

7.000

8.000

Figure E-8. Velocity flow field vectors inside the Inspired Pediatric VAD V3 impeller eye at four flow and pressure conditions. 


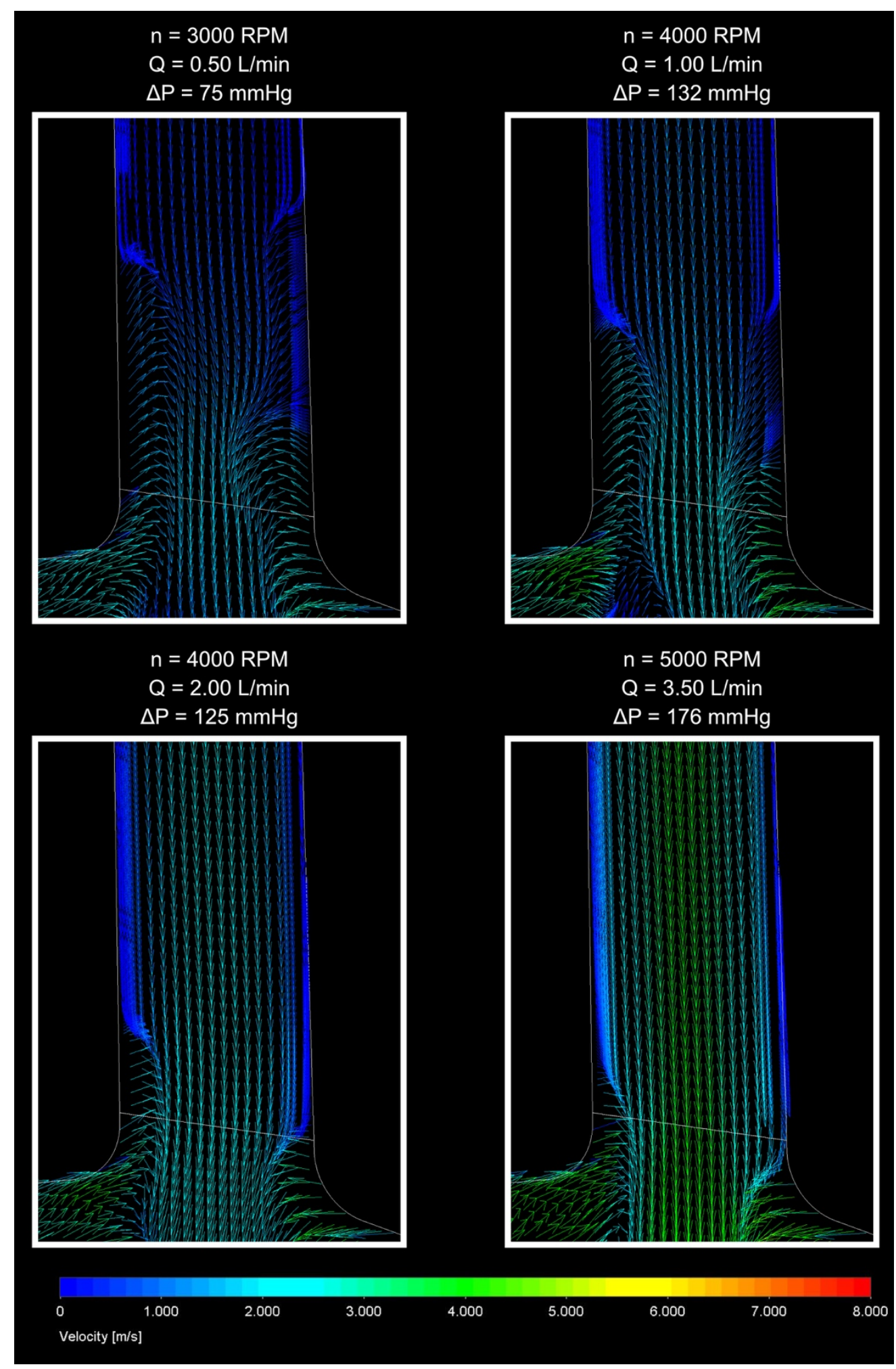

Figure E-9. Velocity flow field vectors at the Inspired Pediatric VAD V3 inlet at four flow and pressure conditions. 
APPENDIX F: VAD V3 ADDITIONAL SIMULATIONS

\section{F.1 V3 Computational Fluid Dynamics (CFD) Simulation - Newtonian Fluid}

For comparative purposes, additional CFD simulations of the Inspired Pediatric VAD V3 were conducted assuming laminar flow and blood as a Newtonian fluid with a density of $\rho=1060 \mathrm{~kg} / \mathrm{m}^{3}$ at $37^{\circ} \mathrm{C}$ and a viscosity of $\mu=$ $0.0035 \mathrm{~Pa}-\mathrm{s}$, for all flow cases at 5000 RPM. Previous CFD analysis of the VAD V2 were performed assuming blood as a Newtonian fluid because, although blood is considered a non-Newtonian fluid, it is shear thinning, and at the high shear rates $\left(>100 \mathrm{~s}^{-1}\right)$ typically found in rotary blood pumps, it behaves as an incompressible Newtonian fluid [64]. This assumption has been used in many previous related CFD studies [65]-[67].

\footnotetext{
F.2 V3 Computational Fluid Dynamics (CFD) Simulation - Turbulent Flow

Since a portion of the flow within the VAD was predicted to be in the laminar-to-turbulent transition range for some cases, additional simulations were performed using a turbulent flow model. SolidWorks Flow Simulation uses a version of the k- $\varepsilon$ turbulence model to solve the Reynolds Averaged NavierStokes equations [99].
} 
Comparison of generated head pressure and hydraulic torque values at 5000

RPM for laminar Newtonian and non-Newtonian solvers and turbulent flow modeling are shown in Figure F-1 and Table F-1.

Table F-1. Computationally predicted hydrodynamic values at 5000 RPM for CFD simulation cases: laminar flow, non-Newtonian fluid; laminar flow, Newtonian fluid; turbulent flow, Newtonian fluid.

\begin{tabular}{|c|c|c|c|c|c|c|c|c|}
\hline \multicolumn{9}{|c|}{ Laminar, Non-Newtonian, 37 C - 5000 RPM } \\
\hline \multirow{2}{*}{$\begin{array}{l}\text { Speed } \\
\text { (RPM) }\end{array}$} & \multirow{2}{*}{$\begin{array}{l}\text { VAD Flow } \\
\text { (L/min) }\end{array}$} & \multirow{2}{*}{$\begin{array}{c}\mathrm{P} 1 \\
(\mathrm{mmHg})\end{array}$} & \multirow{2}{*}{$\begin{array}{c}\mathrm{P} 2 \\
(\mathrm{mmHg})\end{array}$} & \multirow{2}{*}{$\begin{array}{c}\text { Head } \\
(\mathrm{mmHg})\end{array}$} & \multirow{2}{*}{$\begin{array}{c}\text { Hydraulic } \\
\text { Torque } \\
(\mathrm{mNm})\end{array}$} & \multicolumn{3}{|c|}{ Forces $(\mathrm{N})$} \\
\hline & & & & & & $x$ & Y & Z \\
\hline 5000 & 1.00 & -10.82 & 200.00 & 210.82 & 18.07 & -0.07 & 0.43 & 0.46 \\
\hline 5000 & 2.50 & -80.81 & 119.47 & 200.28 & 19.62 & 0.00 & 0.73 & 1.13 \\
\hline 5000 & 3.50 & 13.47 & 200.00 & 186.53 & 19.89 & 0.41 & 1.10 & 1.85 \\
\hline 5000 & 4.00 & 21.06 & 200.00 & 178.94 & 20.36 & 0.27 & 1.17 & 2.22 \\
\hline \multicolumn{9}{|c|}{ Laminar, Newtonian, 37 C - 5000 RPM } \\
\hline \multirow{2}{*}{$\begin{array}{l}\text { Speed } \\
\text { (RPM) }\end{array}$} & \multirow{2}{*}{$\begin{array}{l}\text { VAD Flow } \\
\text { (L/min) }\end{array}$} & \multirow{2}{*}{$\begin{array}{c}\mathrm{P} 1 \\
(\mathrm{mmHg})\end{array}$} & \multirow{2}{*}{$\begin{array}{c}\mathrm{P} 2 \\
(\mathrm{mmHg})\end{array}$} & \multirow{2}{*}{$\begin{array}{l}\text { Head } \\
(\mathrm{mmHg})\end{array}$} & \multirow{2}{*}{$\begin{array}{c}\text { Hydraulic } \\
\text { Torque } \\
(\mathrm{mNm})\end{array}$} & \multicolumn{3}{|c|}{ Forces $(\mathrm{N})$} \\
\hline & & & & & & $x$ & $Y$ & z \\
\hline 5000 & 1.00 & -7.22 & 200.00 & 207.22 & 13.08 & -0.02 & 0.41 & 1.74 \\
\hline 5000 & 2.50 & 2.20 & 200.00 & 197.80 & 14.55 & -0.03 & 0.74 & 2.04 \\
\hline 5000 & 3.50 & 13.59 & 200.00 & 186.41 & 15.27 & 0.13 & 1.25 & 2.63 \\
\hline 5000 & 4.00 & 20.37 & 200.00 & 179.63 & 15.50 & 0.10 & 1.36 & 3.04 \\
\hline \multicolumn{9}{|c|}{ Turbulent, Newtonian, 37 C - 5000 RPM } \\
\hline \multirow{2}{*}{$\begin{array}{l}\text { Speed } \\
\text { (RPM) }\end{array}$} & \multirow{2}{*}{$\begin{array}{l}\text { VAD Flow } \\
\text { (L/min) }\end{array}$} & \multirow{2}{*}{$\begin{array}{c}\mathrm{P} 1 \\
(\mathrm{mmHg})\end{array}$} & \multirow{2}{*}{$\begin{array}{c}\mathrm{P} 2 \\
(\mathrm{mmHg})\end{array}$} & \multirow{2}{*}{$\begin{array}{c}\text { Head } \\
(\mathrm{mmHg})\end{array}$} & \multirow{2}{*}{$\begin{array}{c}\text { Hydraulic } \\
\text { Torque } \\
(\mathrm{mNm})\end{array}$} & \multicolumn{3}{|c|}{ Forces $(\mathrm{N})$} \\
\hline & & & & & & $x$ & $Y$ & Z \\
\hline 5000 & 1.00 & -31.21 & 200.00 & 231.21 & 11.52 & -0.21 & 0.28 & 0.33 \\
\hline 5000 & 2.50 & -22.00 & 200.00 & 222.00 & 13.03 & 0.57 & 0.88 & 1.12 \\
\hline 5000 & 3.50 & -4.55 & 200.00 & 204.55 & 13.92 & 0.14 & 1.34 & 1.46 \\
\hline 5000 & 4.00 & 2.83 & 200.00 & 197.17 & 14.33 & 0.23 & 1.49 & 1.63 \\
\hline
\end{tabular}




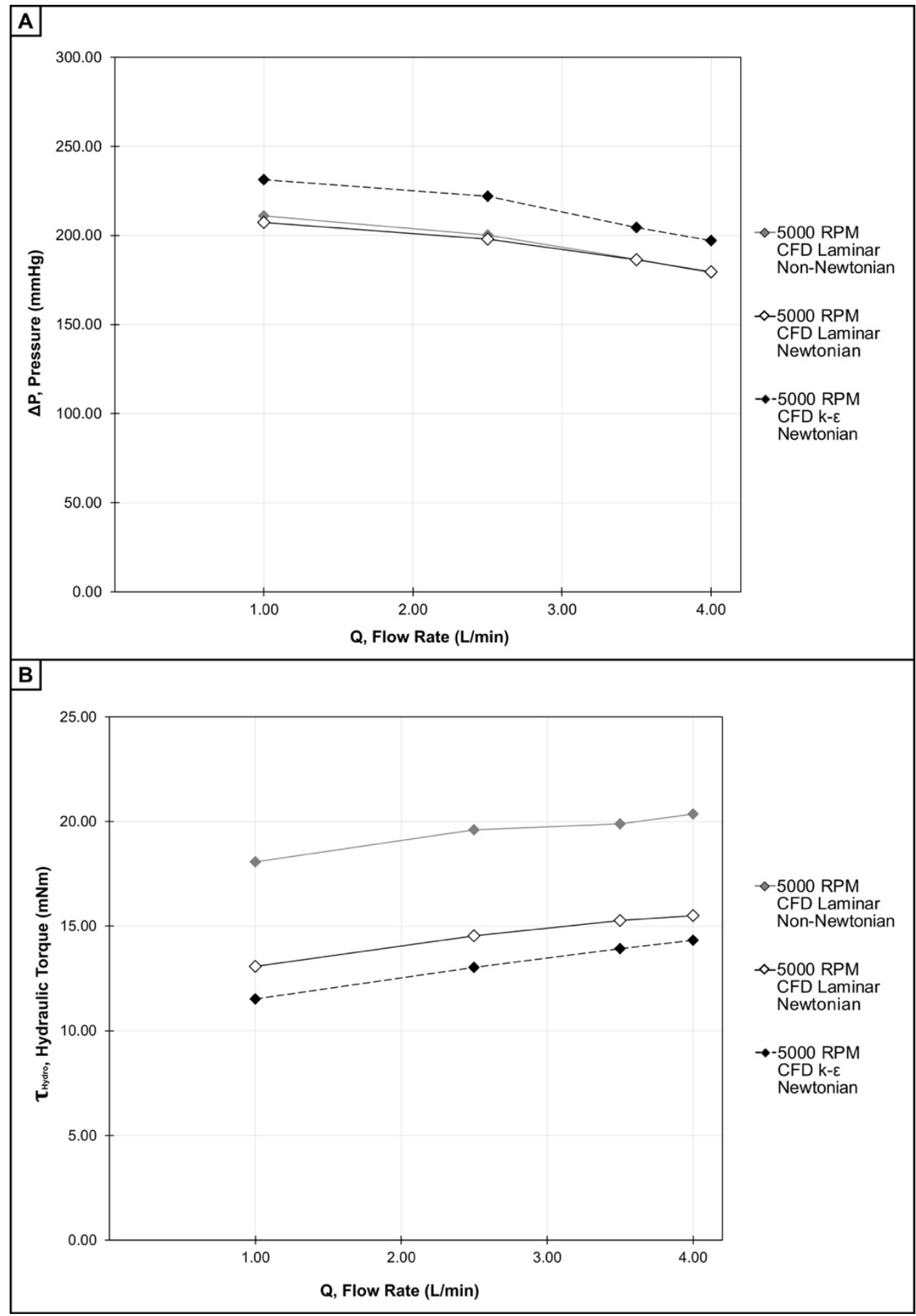

Figure F-1. A) Computationally predicted characteristic pressure-flow curves $(\mathrm{H}$ Q) and, B) computationally predicted impeller/rotor hydraulic torque values at 5000 RPM for CFD simulation cases: laminar flow, nonNewtonian fluid; laminar flow, Newtonian fluid; turbulent flow, Newtonian fluid. 
APPENDIX G: NOMENCLATURE

\section{Abbreviations}

\begin{tabular}{|c|c|c|c|}
\hline BiVAD & Bi-Ventricular & RBP & Rotary Blood Pump \\
\hline & Support/Assist Device & RVAD & $\begin{array}{l}\text { Right Ventricular Assist } \\
\text { Device }\end{array}$ \\
\hline BTT & Bridge to Transplant & SAM & Simple Arc Method \\
\hline CAD & Computer Aided Design & SFL & Static Flow Loop \\
\hline CF & Continuous Flow & SLA & Stereolithography \\
\hline CFD & Computational Fluid & VAD & Ventricular Assist Device \\
\hline CPB & $\begin{array}{l}\text { Dynamics } \\
\text { Cardiopulmonary Bypass }\end{array}$ & VCA & $\begin{array}{l}\text { Voice Coil Magnetic } \\
\text { Actuator }\end{array}$ \\
\hline CV & Cubic Mesh Cell Volume & vWF & von Willebrand Factor \\
\hline DT & Destination Therapy & $\mathrm{V} 1$ & Inspired Pediatric VAD \\
\hline ECMO & $\begin{array}{l}\text { Extracorporeal Membrane } \\
\text { Oxygenation }\end{array}$ & \multirow[t]{2}{*}{ V2 } & \multirow{2}{*}{$\begin{array}{l}\text { Inspired Pediatric VAD } \\
\text { Version } 2\end{array}$} \\
\hline EMF & Electromotive Force & & \\
\hline $\mathrm{HF}$ & Heart Failure & V3 & $\begin{array}{l}\text { Inspired Pediatric VAD } \\
\text { Version } 3\end{array}$ \\
\hline $\begin{array}{l}\mathrm{HI} \\
\mathrm{H}-\mathrm{O}\end{array}$ & $\begin{array}{l}\text { Hemolysis Index } \\
\text { Pressure-Flow Curve }\end{array}$ & \multirow[t]{9}{*}{ WSS } & \multirow[t]{9}{*}{ Wall Shear Stress } \\
\hline ICU & Intensive Care Unit & & \\
\hline LVAD & $\begin{array}{l}\text { Left Ventricular Assist } \\
\text { Device }\end{array}$ & & \\
\hline MagLev & Magnetic Levitation & & \\
\hline MCS & $\begin{array}{l}\text { Mechanical Circulatory } \\
\text { Support }\end{array}$ & & \\
\hline OR & Operating Room & & \\
\hline PIV & $\begin{array}{l}\text { Particle Image } \\
\text { Velocimetry }\end{array}$ & & \\
\hline PMB & Passive Magnetic Bearing & & \\
\hline PMDC & $\begin{array}{l}\text { Permanent Magnet Direct } \\
\text { Current }\end{array}$ & & \\
\hline
\end{tabular}


Variables

\begin{tabular}{|c|c|c|c|}
\hline$a_{1}$ & $\begin{array}{l}\text { Cross-Sectional Flow } \\
\text { Area at Blade Entrance }\end{array}$ & $\mathrm{C}_{2}^{\prime}$ & $\begin{array}{l}\text { Actual Discharge Fluid } \\
\text { Absolute Velocity }\end{array}$ \\
\hline$a_{2}$ & $\begin{array}{l}\text { Cross-Sectional Flow } \\
\text { Area at Blade Discharge }\end{array}$ & $\mathrm{C}_{\mathrm{m} 2}$ & $\begin{array}{l}\text { Discharge Fluid } \\
\text { Meridional Velocity }\end{array}$ \\
\hline$\alpha_{1}$ & Fluid Entrance Angle & $\mathrm{C}_{\mathrm{u} 2}$ & $\begin{array}{l}\text { Discharge Fluid } \\
\text { Tangential Velocity }\end{array}$ \\
\hline $\begin{array}{l}\alpha_{2} \\
\alpha_{2}\end{array}$ & Actual Fluid Discharge & $\mathrm{C}_{\mathrm{u} 2^{\prime}}$ & $\begin{array}{l}\text { Actual Discharge Fluid } \\
\text { Tangential Velocity }\end{array}$ \\
\hline$A_{\text {th }}$ & $\begin{array}{l}\text { Angle } \\
\text { Volute Throat Area }\end{array}$ & $\mathrm{C}_{\mathrm{a}}$ & $\begin{array}{l}\text { Auxiliary Circle, Simple } \\
\text { Arc Method }\end{array}$ \\
\hline$b_{1}$ & $\begin{array}{l}\text { Impeller Blade Entrance } \\
\text { Height }\end{array}$ & $\mathrm{D}_{1}$ & $\begin{array}{l}\text { Impeller Inner/Eye } \\
\text { Diameter }\end{array}$ \\
\hline$b_{2}$ & $\begin{array}{l}\text { Impeller Blade Discharge } \\
\text { Height }\end{array}$ & $\mathrm{D}_{2}$ & Impeller Outer Diameter \\
\hline $\mathrm{B}_{\mathrm{m}}$ & $\begin{array}{l}\text { Motor Viscous Damping } \\
\text { Coefficient }\end{array}$ & $\begin{array}{l}D_{3} \\
d_{1}\end{array}$ & $\begin{array}{l}\text { Volute Base Circle } \\
\text { Diameter of Auxiliary }\end{array}$ \\
\hline$\beta_{1}$ & $\begin{array}{l}\text { Impeller Blade Entrance } \\
\text { Angle }\end{array}$ & e & $\begin{array}{l}\text { Circle, Simple Arc Method } \\
\text { Impeller Blade Thickness }\end{array}$ \\
\hline$\beta_{1}$ & $\begin{array}{l}\text { Actual Impeller Blade } \\
\text { Entrance Angle }\end{array}$ & Force, $\mathrm{X}$ & $\begin{array}{l}\text { Impeller/Rotor Tangential } \\
\text { Force }\end{array}$ \\
\hline$\beta_{2}$ & $\begin{array}{l}\text { Impeller Blade Discharge } \\
\text { Angle }\end{array}$ & Force, $\mathrm{Y}$ & $\begin{array}{l}\text { Impeller/Rotor Radial } \\
\text { Force }\end{array}$ \\
\hline$\beta_{2}$ & $\begin{array}{l}\text { Actual Impeller Blade } \\
\text { Discharge Angle }\end{array}$ & $\begin{array}{l}\text { Force, } Z \\
\text { g }\end{array}$ & $\begin{array}{l}\text { Impeller/Rotor Axial Force } \\
\text { Gravitational Acceleration }\end{array}$ \\
\hline$\beta_{m}$ & $\begin{array}{l}\text { Mean Impeller Blade } \\
\text { Angle }\end{array}$ & $\begin{array}{l}\mathrm{H}_{1} \\
\mathrm{I}\end{array}$ & $\begin{array}{l}\text { Rotor Height } \\
\text { Motor Current }\end{array}$ \\
\hline $\mathrm{C}_{1}$ & $\begin{array}{l}\text { Entrance Fluid Absolute } \\
\text { Velocity }\end{array}$ & $\mathrm{K}_{\mathrm{m} 1}$ & Entrance Speed Constant \\
\hline $\mathrm{C}_{1}^{\prime}$ & $\begin{array}{l}\text { Actual Entrance Fluid } \\
\text { Absolute Velocity }\end{array}$ & $\mathrm{K}_{\mathrm{m} 2}$ & $\begin{array}{l}\text { Discharge Speed } \\
\text { Constant }\end{array}$ \\
\hline $\mathrm{C}_{\mathrm{m} 1}$ & $\begin{array}{l}\text { Entrance Fluid Meridional } \\
\text { Velocity }\end{array}$ & $\mathrm{K}_{\mathrm{e}}$ & $\begin{array}{l}\text { Motor Back Electromotive } \\
\text { Force Constant }\end{array}$ \\
\hline $\mathrm{C}_{\mathrm{u} 1}$ & $\begin{array}{l}\text { Entrance Fluid Tangential } \\
\text { Velocity }\end{array}$ & $\mathrm{K}_{\mathrm{t}}$ & $\begin{array}{l}\text { Implied Motor Torque } \\
\text { Constant }\end{array}$ \\
\hline $\mathrm{C}_{\mathrm{u} 1}{ }^{\prime}$ & $\begin{array}{l}\text { Actual Entrance Fluid } \\
\text { Tangential Velocity }\end{array}$ & $\begin{array}{l}\mu \\
\mathrm{n}\end{array}$ & $\begin{array}{l}\text { Blood Viscosity } \\
\text { Rotational Speed }\end{array}$ \\
\hline $\mathrm{C}_{2}$ & $\begin{array}{l}\text { Discharge Fluid Absolute } \\
\text { Velocity }\end{array}$ & $\begin{array}{l}\eta_{\mathrm{H}} \\
\eta_{\mathrm{s}}\end{array}$ & $\begin{array}{l}\text { Pump Hydraulic Efficiency } \\
\text { Pump Specific Speed }\end{array}$ \\
\hline
\end{tabular}




\begin{tabular}{|c|c|c|c|}
\hline$\Delta \mathrm{P}$ & Head Pressure & $\tau_{\text {Hydro }}$ & Impeller Hydraulic Torque \\
\hline P1 & Pressure at Pump Outlet & $\tau_{\text {Total, Dry }}$ & Impeller Total Torque, Dry \\
\hline P2 & Pressure at Pump Inlet & & System \\
\hline$P_{1 \mathrm{~s}}$ & Blade Pitch Per Second & $\tau_{\text {Total }}$ & Impeller Total Torque \\
\hline$\rho$ & Blood Density & $\mathrm{u}_{1}$ & $\begin{array}{l}\text { Entrance Impeller Blade } \\
\text { Tip Speed }\end{array}$ \\
\hline $\begin{array}{l}Q \\
R\end{array}$ & $\begin{array}{l}\text { Flow Rate } \\
\text { Mean Blade Arc Radius, }\end{array}$ & $\mathrm{u}_{2}$ & $\begin{array}{l}\text { Discharge Impeller Blade } \\
\text { Tip Speed }\end{array}$ \\
\hline $\operatorname{Re}$ & $\begin{array}{l}\text { Simple Arc IVietnod } \\
\text { Reynolds Number }\end{array}$ & $v$ & Fluid Velocity Magnitude \\
\hline $\mathrm{R}_{1}$ & & $\omega$ & Motor Rotational Speed \\
\hline $\begin{array}{l}n 1 \\
s\end{array}$ & Slin Coefficient & $Y$ & Pump Specific Work \\
\hline SG & Specific Gravity of Blood & Z & $\begin{array}{l}\text { Number of Impeller } \\
\text { Blades }\end{array}$ \\
\hline $\mathrm{T}_{\mathrm{fm}}$ & Motor Kinetic Friction & $\delta$ & Pump Diameter Number \\
\hline$\Delta \mathrm{t}_{\mathrm{a}}$ & Time Step (Method a) & $\sigma$ & Pump Speed Number \\
\hline$\Delta \mathrm{t}_{\mathrm{b}}$ & Time Step (Method b) & $\psi$ & Pump Head Coefficient \\
\hline$\tau$ & $\begin{array}{l}\text { Scalar Representative } \\
\text { Viscous Shear Stress }\end{array}$ & $\phi$ & Pump Flow Coefficient \\
\hline$\tau_{\mathrm{DC}}$ & DC Motor Torque & $\dot{\gamma}$ & Shear Rate \\
\hline ricti & Impeller Frictional Torque & & \\
\hline
\end{tabular}




\section{CURRICULUM VITAE}

Landon Heath Tompkins

landon.tompkins@louisville.edu

DOB January $14^{\text {th }}, 1988$ - Louisville, Kentucky

\section{EDUCATION}

$2015-2020$

Ph.D., Doctor of Philosophy in Interdisciplinary

Studies with a specialization in Translational

Bioengineering

J.B. Speed School of Engineering,

University of Louisville, Louisville, $K Y$

$2010-2011 \quad$ MEng., Master of Engineering with a specialization in Mechanical Engineering

J.B. Speed School of Engineering,

University of Louisville, Louisville, $K Y$

$2006-2010 \quad$ B.S., Bachelor of Science in Mechanical

Engineering

J.B. Speed School of Engineering,

University of Louisville, Louisville, $K Y$

\section{ACADEMIC AND PROFESSIONAL EXPERIENCE}

Sept. 2018 - Present Co-Owner / Director of Engineering

Myocardial Assist Systems \& Technology, MAST LLC

Louisville, $K Y$

Sept. 2018 - Present Biomedical Research Engineer

Cor Habere Corp.

Louisville, KY

Aug. 2015 - Sept. 2018 Graduate Research Assistant 
Advanced Heart Failure Research Group,

Cardiovascular Innovation Institute

University of Louisville

Louisville, $K Y$

Nov. 2013 - May 2015 Chief Technology Officer

SCR Inc.

Louisville, $K Y$

Aug. 2009 - Nov. 2013 Senior Engineer

SCR Inc.

Louisville, $K Y$

Jan. 2008 - Aug. 2009 Aircraft Performance Engineering Co-op

United Parcel Service, Airline Division

Louisville, $K Y$

\section{PROFESSIONAL AFFILIATIONS}

Member

Member

Past Member

Past Member
American Society of Artificial Internal Organs (ASAIO)

ASAIOfyi for Young Innovators

Biomedical Engineering Society (BMES)

American Heart Association (AHA)

\section{AWARDS AND RECOGNITION}

2020

2019

2019

2018

2013

UofL Doctoral Student Inventorship Award

J.B. Speed School of Engineering, Department of Bioengineering

\section{UofL APPKI Doctoral Student Exemplary} Achievement Award

For Exemplary Research Achievement in Bioengineering

ASAIOfyi Fellowship Award

ASAIO $7^{\text {th }}$ Annual Medical Device Entrepreneur's Forum

$1^{\text {st }}$ Place Presentation for Uniti Connect, MAST Inc.

J.B. Speed School Outstanding Corporate Partner Award 
For Student Co-op Development and Mentorship, SCR Inc.

2005

Eagle Scout

2005

Yarmuth Book Award

UofL Academic Achievement Recognition

\section{PEER-REVIEWED PUBLICATIONS}

1. Warren S, GA Giridharan, RD Dowling, PA Spence, L Tompkins, E Gratz, LC Sherwood, MA Sobieski, CR Bartoli, MS Slaughter, RS Keynton, and SC Koenig. Feasibility of Subcutaneous ECG leads for synchronized timing of a counterpulsation device. Cardiovascular Engineering and Technology, 3(1):17-25, 2012

2. DuPont WH, Meuris BJ, Hardesty VH, Barnhart EC, Tompkins LH, Golden MJP, Usher CJ, Spence PA, Caldwell LK, Post EM, Beeler MK, Kraemer WJ. The Effects Combining Cryocompression Therapy following an Acute Bout of Resistance Exercise on Performance and Recovery. Journal of Sports Science and Medicine. (2017) Aug 8;16(3):333-342.

3. Carnahan, S. R., Koenig, S. C., Sobieski, M. A., Schumer, E. M., Monreal, G., Wang, Y., Choi, Y., Meuris, B. J., Tompkins, L. H., Wu, Z. J., Slaughter, M. S., Giridharan, G. A. (2017). Efficacy of Subcutaneous Electrocardiogram Leads for Synchronous Timing During Chronic Counterpulsation Therapy. ASAIO Journal, 63(2), 134-138.

4. Tompkins, L. H., Gellman, B. N., Morello, G. F., Prina, S. R., Roussel, T., Kopechek, J. A., Petit, P. C., Slaughter, M. S., Koenig, S. C., Dasse, K. A. (2020). Design and Initial Computational Evaluation of a Pediatric MagLev Rotary Blood Pump. ASAIO Journal (Accepted for Publications, September 2020)

\section{PEER-REVIEWED CONFERENCE ABSTRACTS}

1. N. R. Tapolsky, L. H. Tompkins, C. J. Smith, N. R. Ebersold, M. A. Sobieski, G. Monreal, L. Polverelli, P. Monticone, C. Botterbusch, M. S. Slaughter, S. C. Koenig, In vitro Performance of a Novel Membraneoscillating Left Ventricular Assist Device. ASAIO 63rd ANNUAL CONFERENCE, Chicago, IL, 2017

2. A. Kanukunta, M. Slaughter, L. Tompkins, T. Adams, M. Sobieski, S. Koenig, J. Joly, R. Bourge, Artifact in Pressure Measurement with 
Pulmonary Artery Pressure Catheters. Biomedical Engineering Society (BMES) 50th Annual Meeting, Atlanta, GA, 2018

3. G. Giridharan, M. Sobieski, G. Monreal, L. Tompkins, T. Adams, E. Schumer, W. Whited, M. Gallo, M. Ising, J. Jimenez, S. Koenig, M. Slaughter, Left Atrial Appendage (LAA) Device as A Non-Valvular Atrial Fibrillation (NVAF) Therapy. Biomedical Engineering Society (BMES) 50th Annual Meeting, Atlanta, GA, 2018

4. L. Tompkins, S. Koenig, M. Sobieski, G. Koenig, T. Adams, G. Monreal, B. Gellman, P. Petit, K. Dasse, M. Slaughter, Development of a Sutureless LVAD Outflow Graft Anastomotic Quick-connect system. ASAIO 65th ANNUAL CONFERENCE, San Francisco, CA, 2019

5. R. Wampler, B. Hull, J. Karlen, L. Tompkins, T. Adams, S. Koenig, M. Slaughter, HVAD Intelligent Control for Dynamic Ventricular Unloading. ASAIO 65th ANNUAL CONFERENCE, San Francisco, CA, 2019

6. L. Tompkins, B. Gellman, T. Adams, K. Dasse, S. Koenig, Novel Centrifugal Impeller Design for a MagLev Pediatric Cardiac Assist Pump. ASAIO 66th ANNUAL CONFERENCE, Chicago, IL, (Virtual Conference), 2020

\section{CONFERENCE PRESENTATIONS}

1. Tompkins L. H., Uniti Connect: Sutureless Anastomotic System. 7th Annual Medical Device Entrepreneur's Forum, ASAIO 64th ANNUAL CONFERENCE, Washington, D.C., 2018

2. Tompkins L. H., Development of a Sutureless LVAD Outflow Graft Anastomotic Quick-connect system. ASAIO 65th ANNUAL CONFERENCE, San Francisco, CA, 2019

3. Tompkins L. H., Novel Centrifugal Impeller Design for a MagLev Pediatric Cardiac Assist Pump. ASAIO 66th ANNUAL CONFERENCE, Chicago, IL, (Virtual Conference), 2020 
ACTIVE FUNDED GRANTS

R43HL152894-01 (PI MAST: Tompkins, co-PI UofL: Koenig, Slaughter)

8/21/2020 - 9/20/2021

$\$ 278,487$

NIH SBIR phase I Grant (MAST, Louisville KY)

Development of a partial occlusion device to aid coring and anastomosis of the aorta

The major goal of this project is to demonstrate feasibility of a partial occlusion device (CardiAction) to facilitate minimally invasive surgery for implant of mechanical circulatory support (MCS) devices.

Role: Principle Investigator ( $40 \%$ effort)

R43HL142385-01 (PI MAST: Tompkins, co-PI UofL: Koenig, Slaughter)

9/24/2018 - 8/30/2021

$\$ 303,817$

NIH SBIR phase I Grant (MAST, Louisville KY)

Development of a sutureless LVAD outflow graft anastomotic quick-connect system

The major goal of this project is to demonstrate feasibility of a sutureless graft to aorta anastomotic device (Uniti Connect) to facilitate minimally invasive implantation of mechanical circulatory support (MCS) devices.

Role: Principle Investigator (40\% effort)

R43HL144214-01 (PI IT: Dasse, co-PI UofL: Koenig, Slaughter)

$7 / 1 / 2018-6 / 30 / 2021$

$\$ 415,921$

NIH SBIR phase I Grant (Inspired Therapeutics, Merritt Island FL)

Development of a universal maglev driver for five pediatric cardiac and respiratory applications

The major goal of this project is to demonstrate feasibility of pediatric Universal MagLev system designed to provide up to 30-days of mechanical circulatory support (MCS) as left ventricular assist device (LVAD).

Role: Doctoral Student, Tompkins - (10\% effort)

R43HL142337-01 (PI CH: Jimenez, co-PI UofL: Koenig, Slaughter)

9/24/2018-8/30/2021

$\$ 311,131$ 
NIH SBIR phase I Grant (Cor Habere, Louisville KY)

Left Atrial Appendage (LAA) closure device for stroke prevention in patients with AFIB

The major goal of this project is to complete pre-clinical development of a left atrial appendage (LAA) closure device (StrokeShield) designed to prevent strokes in patients with atrial fibrillation (AFIB).

Role: CH Engineer, Tompkins (10\% effort)

\section{COMPLETED GRANTS}

R44HL123120-01 (PI: Spence)

05/01/2014 - 04/30/2015

$\$ 320,765$

NIH SBIR Phase I \& II Fast Track (SCR Inc., Louisville KY)

Development of SVAD System for HF Therapy

The objective of this proposal was to complete the engineering development and pre-clinical testing of the SVAD system (SCR, Louisville KY \& Heartware Inc., Framingham MA) to provide partial cardiac assist in patients with less advanced stage heart failure (HF).

Role: Co-Investigator, Tompkins

KSTC-184-512-13-153 (PI: Spence)

01/01/2013 - 12/31/2015

Kentucky Science and Technology Corporation (KSTC) Grant (SCR Inc., Louisville KY)

Counterpulsation device with integrated ECG sensing

The major goal of this proposal was to complete development of the Symphony system (Abiomed, Danvers MA) by integrating a subcutaneous lead system for ECG detection, signal conditioning, and control.

Role: Co-Investigator, Tompkins

2R43HL102981-02A (PI: Spence)

04/01/2012 - 03/31/2014

NIH SBIR Phase II grant (SCR Inc., Louisville KY)

Subcutaneous ECG Sensing 
The major goal of this project was to complete development of novel subcutaneous ECG leads integrated with the Symphony device (Abiomed, Danvers MA) and portable driver.

Role: SCR Engineer, Tompkins

2R44HL088760-02 (PI: Spence)

05/15/2009 - 04/12/2012

$\$ 1,580,486$

NIH SBIR Phase II Grant (SCR Inc., Louisville KY)

Portable pneumatic driver for counterpulsation therapy

The major goals of this project were to (1) complete engineering development, (2) demonstrate reliability and hemocompatibility, and (3) demonstrate safety and biocompatibility of a portable pneumatic driver for a novel counterpulsation device (CPD) to treat early stage heart failure.

Role: SCR Engineer, Tompkins

2R44HL083586-02A1 (PI: Spence)

08/01/2008 -07/31/2011

$\$ 1,440,170$

NIH SBIR Phase II Grant (SCR Inc., Louisville KY)

Development of a Counterpulsation Therapy Device

The major goals of this project were to (1) complete engineering development and surgical procedures, (2) demonstrate reliability and hemocompatibility, and (3) demonstrate safety and biocompatibility of a novel counterpulsation device (CPD) to treat early stage heart failure.

Role: SCR Engineer, Tompkins

R43HL102981 (PI: Spence)

03/01/2010-02/28/2011

NIH SBIR Phase I Grant (SCR Inc., Louisville KY)

Subcutaneous ECG Sensing

The major goal of this project was to demonstrate feasibility of a novel subcutaneous ECG to be integrated with the Symphony device and portable driver.

Role: SCR Engineer, Tompkins 


\section{PATENTS (ISSUED)}

1. Spence, P.A., Warren, S.P., Wells, E.J., Dierking, W.K., Bachman, D.R., and Tompkins, L., Systems, devices and methods for treating the heart with ablation, US Patent US9216055 filed August 2010, Issued December 2015.

2. Spence, P.A., Warren, S.P., Wells, E.J., Dierking, W.K., Bachman, D.R., and Tompkins, L., Systems and methods for treating the heart with ablation, US Patent US9504523 filed August 2012, Issued November 2016.

3. Spence, P.A., Warren, S.P., Wells, E.J., Dierking, W.K., Bachman, D.R., and Tompkins, L., Systems and methods for treating the heart with ablation, US Patent US9713495 filed November 2015, Issued July 2017.

4. Spence, P.A., Tompkins, L., and Acland, R., Apparatus and methods for cutting an atrial wall, US Patent US9808283 filed December 2014, Issued November 2017.

5. Spence, P.A., Tompkins, L., Mitral repair and replacement devices and methods, US Patent US10016272 filed September 2015, Issued July 2018.

6. Spence, P.A., Tompkins, L., Apparatus and methods for implanting a replacement heart valve, US Patent US10034749 filed August 2014, Issued July 2018.

7. Chau, M., Siegel, A., Spence, P.A., Tompkins, L., Coiled anchor for supporting prosthetic heart valve, prosthetic heart valve, and deployment device, US Patent US10052198 filed February 2015, Issued August 2018.

8. Spence, P.A., Tompkins, L., Devices, systems and methods for delivering a prosthetic mitral valve and anchoring device, US Patent US 10052199 filed February 2015, Issued August 2018.

9. Spence, P.A., Tompkins, L., Chau, M., Siegel, A., Replacement heart valve apparatus and methods, US Patent US10226330 filed August 2014, Issued March 2019.

10. Spence, P.A., Tompkins, L., Mitral valve docking devices, systems and methods, US Patent US10226339 filed January 2013, Issued March 2019.

11. Chau, M., Siegel, A., Spence, P.A., Tompkins, L., Coiled anchor for supporting prosthetic heart valve, prosthetic heart valve, and deployment device, US Patent US10588742 filed March 2018, Issued March 2020.

12. Spence, P.A., Tompkins, L., Mitral repair and replacement devices and methods, US Patent US10653519 filed July 2018, Issued May 2020.

13. Spence, P.A., Tompkins, L., and Acland, R., Apparatus and methods for cutting an atrial wall, US Patent US10660669 filed October 2017, Issued May 2020. 


\section{PATENTS (APPLICATIONS)}

1. Spence, P.A., Tompkins, L., Walling, S.C., Hatton, R.D., Hutzenlaub, J., Spence, W., Singer, A. Athletic cooling and heating systems, devices and methods, US Patent App. 14/127,054 filed July 2012. Patent Pending

2. Spence, P.A., Tompkins, L., Walling, S.C., Hatton, R.D., Hutzenlaub, J., Spence, W., Singer, A. Athletic cooling and heating systems, devices and methods, US Patent App. 15/248,674 filed August 2016. Patent Pending

3. L Tompkins, M Sobieski, S Koenig, G Monreal, M Slaughter, Sutureless Graft Anastomotic Quick Connect System, US Provisional Patent Application 62/625,635 (filed February 2018, Patent pending)

4. L Tompkins, M Sobieski, S Koenig, G Monreal, M Slaughter, Sutureless Graft Anastomotic Quick Connect System, PCT International Patent Application PCT/US2019/016285 (filed February 2019, Patent pending)

5. L Tompkins, S Koenig, G Monreal, M Slaughter, F Mayhaus, C Buckley, A Adams, V Sager, Development of a Partial Occlusion Device to Aid in Coring and Anastomosis of the Aorta, US Provisional Patent Application 62/846,210 (filed May 2019, Patent pending)

6. L Tompkins, G Koenig, K Dasse, B Gellman, S Koenig, M Slaughter, Sutureless Graft Anastomotic Quick Connect System with Variable Adjustment, US Provisional Patent Application 64/866,886 (filed June 2019, Patent pending)

7. L Tompkins, S Koenig, G Monreal, M Slaughter, F Mayhaus, C Buckley, A Adams, V Sager, Partial Vessel Occlusion Device, PCT International Patent Application PCT/US2020/031751 (filed May 2020, Patent pending)

8. L Tompkins, M Sobieski, S Koenig, G Monreal, M Slaughter, Sutureless Graft Anastomotic Quick Connect System, US National Stage Patent Application 16/963,789 (filed July 2020, Patent pending)

\section{RESEARCH DISCLOSURES}

1. L Tompkins, M Sobieski, S Koenig, G Monreal, M Slaughter, Development of a sutureless LVAD outflow graft anastomotic quick-connect system, UofL Research Disclosure RDF 18041 (submitted Dec 2017)

2. L Tompkins, S Koenig, G Monreal, M Slaughter, F Mayhaus, C Buckley, A Adams, V Sager, Development of a Partial Occlusion Device to Aid in Coring and Anastomosis of the Aorta, UofL Research Disclosure RDF 19030 (submitted Sep 2018)

3. L Tompkins, M Sobieski, S Koenig, G Monreal, M Slaughter, Development of a sutureless graft anastomotic quick-connect system, UofL Research Disclosure RDF 20079 (submitted June 2020) 